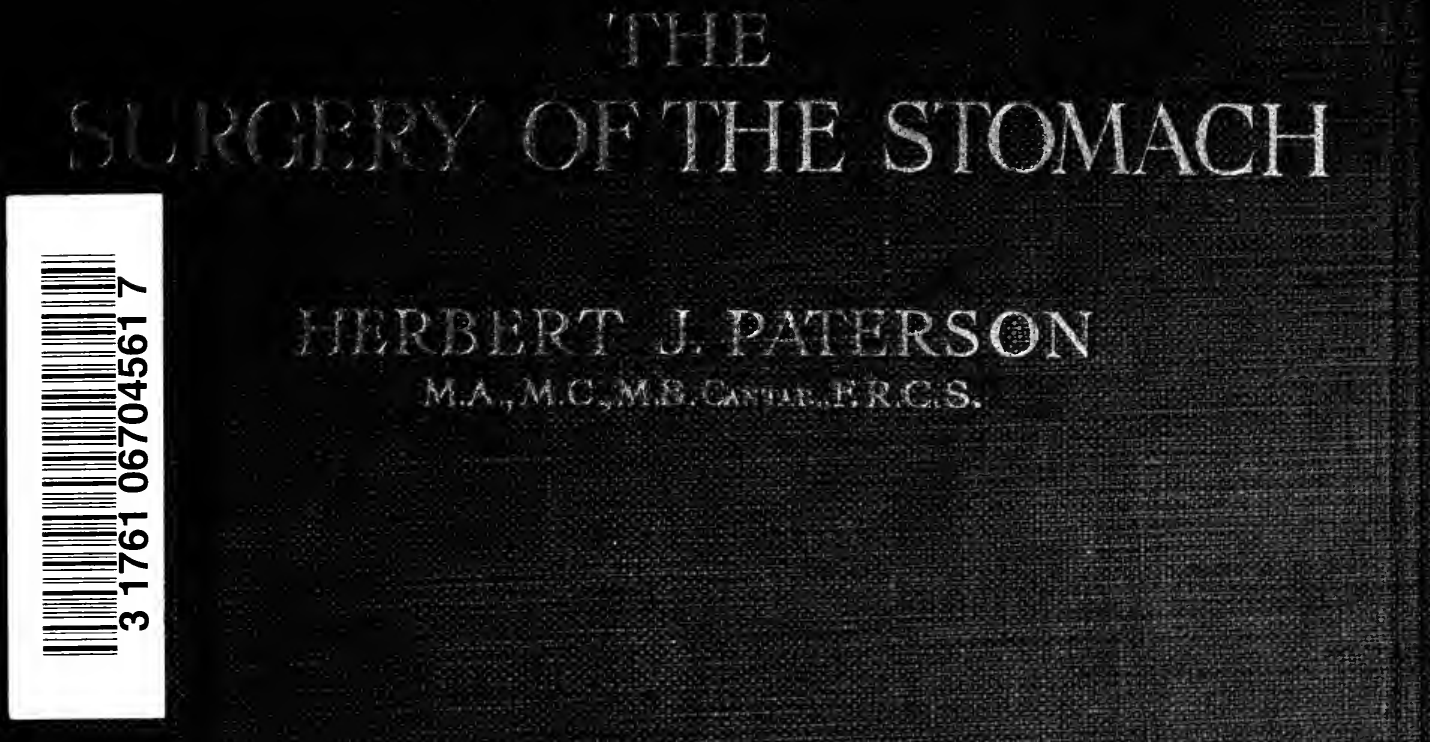

YMRBLRT J. PATHRSON

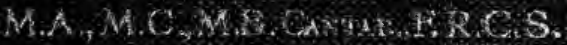




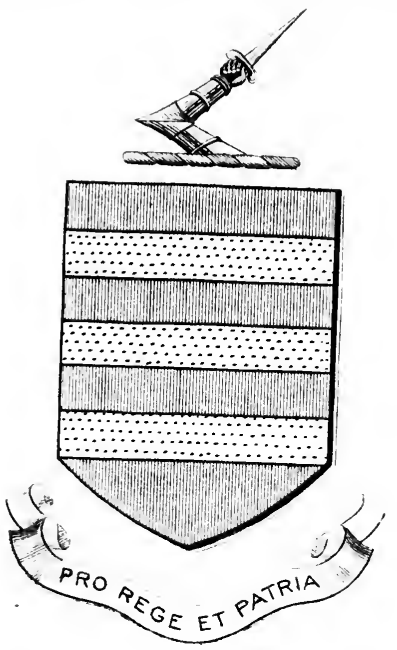

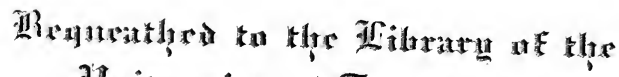
Mniversitu af Taroutu lon

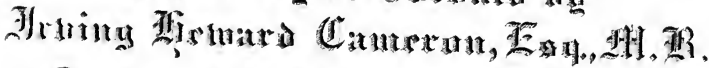

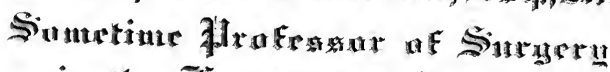

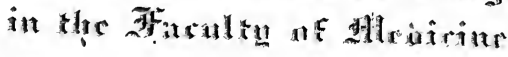






\section{Digitized by the Internet Archive in 2007 with funding from Microsoft Corporation}




\section{THE SURGERY OF THE STOMACH}




\section{WORKS BY HERBER'T J. PATERSON}

GASTRIC SURGERY. London, 1906.

Bailliere, Tindall \& Cox.

JEJUNAL AND GASTRO-JEJUNAL ULCER, 1909.

John BaLe, Sons \& Danielsson.

APPENDICULAR GASTRALGIA, 1910. John Bale, Sons \& Danielsson.

SURGICAL PATHOLOGY.

(Walsham and Paterson.)

Third Edition, 1904. Bailliere, Tindall \& Cox.

THE SURGERY OF THE STOMACH, 1913.

JAMES Nisbet \& Co. 
$\prod_{P}^{M D i}$

\title{
THE SURGERY OF THE STOMACH
}

\section{A HANDBOOK OF DIAGNOSIS AND TREATMENT}

\author{
BY \\ HERBER'T J. PATERSON \\ M.A., M.C., M.B. (CаNTAB.), F.R.C.S. \\ LONDON
}

WITH PLATES

Hondon

JAMES NISBE'T $\xi$ CO., LIMITED

22 BERNERS STREET, W.

1913

[All rights reserved]

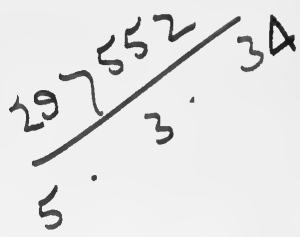


Printed by Ballantrne, Hanson $\delta$ Co. At the Ballantyne Press, Edinburgh 
MY FRIEND

DR. W ILLIA M J. MA Y O

OF ROCHESTER, U.S.A. 



\section{PREFACE}

IN the following pages I have attempted to give a practical account of "The Diagnosis and Treatment of those Affections of the Stomach which are amenable to Direct Surgical Interference." 1 The significance of the information to be gained from "test-meals" is considered in some detail, because I believe that such investigations are of great value in the diagnosis of gastric disease. It appears to me strange, that whereas at the present time, bacteriological and pathological examinations are used so extensively in the diagnosis of disease, chemical methods are so rarely made use of in practice, and dealt with so briefly in text-books. The stomach-tube should be part of the armamentarium of every practitioner. It is not essential that he should perform all the quantitative analyses to which I refer, but I venture to think that he would be repaid amply, if he carried out the ordinary qualitative tests, and the estimation of the total acidity of the gastric contents. The practitioner, be he consultant or otherwise, who, without the aid which such investigations afford, continues to treat a patient suffering from persistent indigestion, or gastric pain, is acting unfairly to his patient and unjustly to himself.

The advances made in recent years by the many brilliant workers in the field of gastric disease, have led to successes which are among the greatest achievements of surgery. Lives are saved and suffering is relieved to an extent undreamt of twenty years ago. But much remains to be

1 The title of the essay for which the Jacksonian Prize (R.C.S.) 1904 was awarded to the author. 
done. The loss of human life from cancer casts a shadow over the triumphs of the past. The saddest and most discouraging part of the work of a surgeon is the frequency with which he sees patients suffering from cancer so advanced, that the time for a radical operation has gone for ever. Especially is this true of gastric cancer. In less than 5 per cent of the cases I see, is a radical operation possible. The stomach is affected with cancer more frequently than any other organ of the body, and yet, partial gastrectomy is one of the uncommon operations of surgery. This reproach to our profession would be removed, in some measure at least, were the use of the stomach-tube part of the routine of gastric diagnosis. On this point I feel strongly, and have no hesitation in saying, that the number of cases of gastric cancer treated successfully by operation, ought to be, and could be, increased greatly, if only the cases were recognised earlier. Gastric cancer, contrary to popular belief, can be treated successfully, and earlier diagnosis is possible, even with the means at present at our disposal.

The operation of gastro-jejunostomy is not a panacea for all the ills to which the stomach is heir, but when performed in cases in which there is a definite organic lesion of the stomach or duodenum, the results are excellent. It is the keystone of gastric surgery.

It has been my aim to present methods of diagnosis and treatment, in a manner which may prove useful in practice to practitioners and students. If in any degree this aim be realised, the time spent by the reader and the author will not have been wasted.

Limitations of space have prevented me from referring to all the views and operative methods of those who have done such admirable work in gastric surgery. While the opinions expressed are based largely on the experience gained from my own failures and successes, doubtless the sub- 
conscious mind is influenced, even more than one realises, by the fruits of the labour of others. It is, therefore, a sincere pleasure to acknowledge the stimulus and inspiration I have derived from perusal of the writings and witnessing the work of many brilliant exponents of our art, especially Dr. Soltau Fenwick, Mr. Arbuthnot Lane, Mr. Rutherford Morison, Sir Berkeley Moynihan, Mr. Harold J. Stiles and Sir J. Bland Sutton in this country, and Drs. W. J. and C. H. Mayo, Dr. J. B. Murphy, Dr. John B. Deaver, Dr. Howard Kelly, and Professor Kocher abroad. With Dr. Fenwick it has been my privilege to be associated during the past eleven years, and it was through him that my interest in gastric surgery was begun and fostered.

I should be indeed ungrateful, if I did not put on record how much I owe to my former teacher, Mr. Harrison Cripps. While I was his house surgeon at St. Bartholomew's and his assistant in private, I had the advantage of helping him in his abdominal operations. The lessons I learnt have been of inestimable value to me in my subsequent work.

To my friend, Mr. Chas. Gibbs, I am indebted for many valuable suggestions, for which I offer him my thanks.

I have also to thank Dr. Fenwick, Dr. Porter Parkinson, and Messrs. Allen and Hanburys for the loan of blocks.

I have been fortunate in my helpers. To my former secretary, Miss Rendle, I am greatly indebted for much help during the preparation of the earlier portions of the work.

Last, but not least, my secretary, Miss Sinzininex, in addition to preparing the index, and sharing with me the correction of the proofs, has given to me constant and valuable assistance at all stages of my task, and I desire to express my sincere thanks.

HERBERT J. PATERSON.

9 Upper Wimpole Street, W. January 1913. 



\section{CON'TENTS}

CHAP.

I. The Method of Investigation of Gastric Cases. . 1

II. The Preparation and Treatment of Patients before Gastric Operations . . . . . . . . 17

III. Operations on the Stomach . . . . . . 21

1. Gastrotomy • • . . . . . . 23

2. Gastrostomy . . . . . . . 25

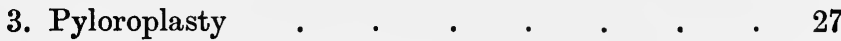

4. Pyloro-gastro-duodenostomy $\quad$. $\quad$. $\quad$. 27

5. Gastro-jejunostomy . . . • . $\quad$. 30

6. Gastro-plasty, gastro-gastrostomy . . . 41

7. Gastrectomy . . . . . . . 42

8. Excision of gastric ulcers $\quad$ • $\quad$. $\quad$. 52

9. Jejunostomy . . $\quad$ • $\quad$ • $\quad$. 53

10. Gastropexy . . . . . . . 55

IV. The Treatment of Patients after Gastric Operations 61

V. Complications after Gastric Operations . . . . 71

VI. The Physiological Effects of the Operation of

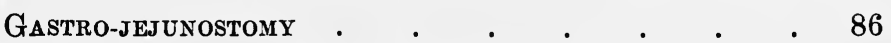

VII. Jejunal and Gastro-jejunal Ulcers . • • . 95

ViII. Gastric Ulcer, its Pathology, Symptoms, and DiagNosis . . . . . . . . . . 115

iX. Duodenal Ulcer, its Pathology, Symptoms, and Diagnosis . . .

X. The Treatment of Gastric and Duodenal Ulcers $\quad 142$

XI. The Complications of Gastric and Duodenal Ulcers-

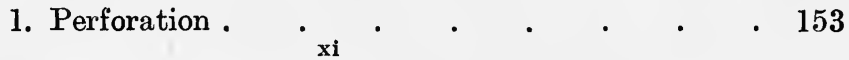


CHAP.

XII. The Complications of Gastric and Duodenal Ulcers, continued-

2. Hæmorrhage . . . . . . . 180

XiII. The Complications of Gastric and Duodenal Ulcers, continued-

3. Chronic dilatation of the stomach

XIV. The Complications of Gastric and Duodenal Ulcers, continued-

4. Hour-glass stomach . . . . . . . . 193

XV. The Complications of Gastric and Duodenal Ulcers, continued-

5. Gastric tetany

XVI. The Pathology, Symptoms, and Diagnosis of Gastric Carcinoma and Sarcoma . . . . . . . 206

XVII. The Treatment of Gastric Carcinoma and Sarcoma . 236

XVIII. Ulcus Carcinomatosum . . . . . . . 248

XIX Infantile Hypertrophic Pyloric Stenosis . . . 254

XX. Fibromatosis of the Stomach . . . . . . . 262

XXI. Benign Tumours of the Stomach . . . . . . 265

XXII. Appendicular Gastralgia . . . . . . . 270

XXIII. Syphilis and Tubercle of the Stomach . . . 281

XXIV. Gastric Atony and Gastroptosis . . . . . 285

Appendix $. \quad . \quad . \quad . \quad . \quad . \quad . \quad . \quad .290$

Including-

Technique of passing Stomach-tube, and Gastric Lavage.

Methods of Gastric Analysis.

The Sero-diagnosis of Cancer.

Skin Reaction in Carcinoma.

Diets, \&c.

List of Authorities . . . . . . . . . 305

INDEX . . . . . . . . . . . 307 


\section{ILLUSTRA'TIONS}

FIGS.

1. "Grip-eyed" needle . . . . . . . . 22

$2 \&$ 3. Gastrostomy . . . . . . . . . To face 26

4 \& 5. Gastrostomy . . . . . . . . " " 26

6 \& 7. Anterior gastro-jejunostomy . . . . . " , 30

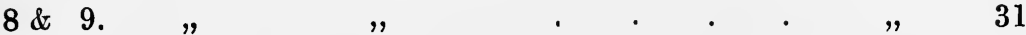

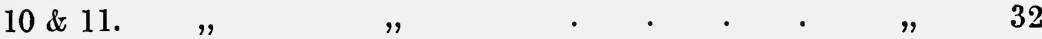

$12 \& 13 . \quad " \quad " \quad$. $\quad$. $\quad . \quad . \quad, \quad, \quad 33$

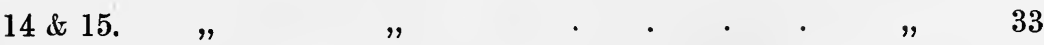

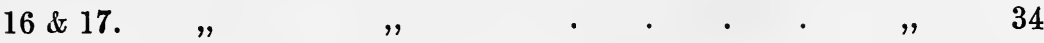

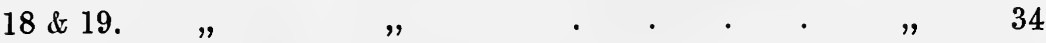

$20 \& 21 . \quad " \quad " \quad$. . . . $\quad, \quad 34$

22. Microphotograph of gastro-jejunostomy opening . 35

$23 \& 24$. Posterior gastro-jejunostomy . . . . . To face 36

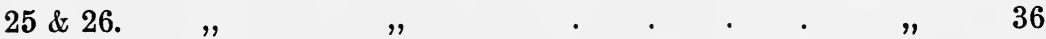

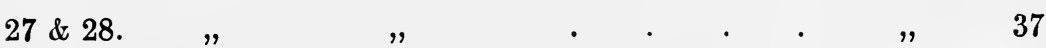

$29 \& 30 . \quad \# \quad, \quad . \quad . \quad . \quad . \quad, \quad, \quad 37$

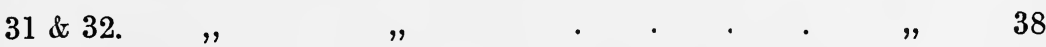

$33 \& 34 . \quad " \quad$ \& $\quad$. . . . . $\quad$. $\quad$, 38

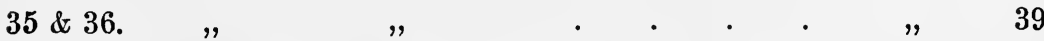

$37 \& 38$. Gastro-jejunostomy " en-Y" . . . . . . 40

39. Line of section in partial gastrectomy . . . . 43

40. Portion of stomach usually resected . . . To face 48

41. Partial gastrectomy . . . . . . " 48

42. Partial gastrectomy completed . . . . " 50

$43 \& 44$. Transgastric resection of gastric ulcer . . $\quad, \quad 53$

45. Sister Agnes' "Bed-stop" . . . . . . . 62 
46. Electrically heated saline can . . . . . . 64

47. Apparatus for proctoclysis . . . . . . . $\quad$. 65

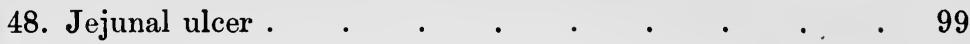

49. Chronic ulcer of stomach . . . . To face 116

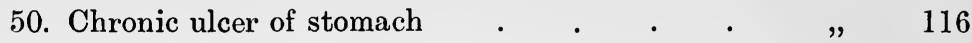

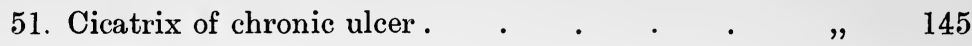

52. Recurrent ulcer in operation scar . . . . ", 145

$53 \& 54$. Acute perforation of gastric ulcer . . . . , , 154

55. The interior of an hour-glass stomach . . . , , 194

56. The exterior of an hour-glass stomach . . , , 194

57. Microphotograph-Spheroidal-celled carcinoma , 207

58. Microphotograph-Cylindrical-celled carcinoma , 207

59. Microphotograph-Carcinoma with colloid

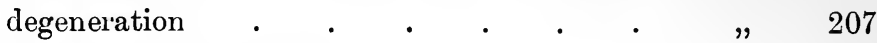

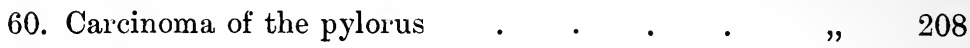

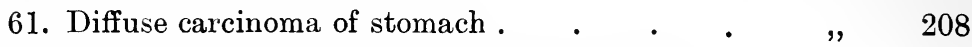

62. Gastric carcinoma invading transverse colon . . . 209

63. The lymphatic glands of the stomach . . . $\quad 210$

64. The areas drained by gastric lymphatic glands To face 210

65. Microphotograph showing islets of cancer cells beyond indurated area $\quad . \quad . \quad . \quad$. $\quad$, $\quad 210$

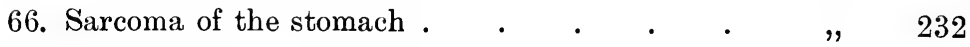

67. Microphotograph-Sarcoma of the stomach . " 232

68. Carcinoma of cardiac end of stomach _ . . $\quad$, $\quad 239$

69. Symonds' tube . . . . . . . . . . 246

70. Infantile pyloric stenosis . . . . To face 254

71. Fibromatosis of the stomach . . . . , , 263

72. Mucous polypi of the stomach • • • • „ 266

73. Pedunculated fibroma of stomach . • • , , 266

74. Senoran's stomach evacuator . . . . . 290 


\section{SURGERY OF THE STOMACH}

\section{CHAPTER I}

\section{THE METHOD OF INYESTIGATION OF GASTRIC CASES}

THE object of this chapter is to give an outline of the method to be pursued in investigating a case of gastric disease, together with a brief summary of the main points of diagnostic importance. For fuller details as to symptoms and diagnosis, the reader is referred to the various chapters dealing with particular lesions.

\section{THE HISTORY}

From a diagnostic point of view the history is of the utmost importance, and great care should be taken to obtain an accurate and exact account of the origin and progress of the patient's illness. The histories recorded in hospital notes usually are so vague and incomplete that they are valueless. Students nowadays are so obsessed with the importance of blood counts, X-ray examinations, \&c., that they are apt to relegate even physical examination to a second place. Laboratory investigations are an aid-and a valuable aid-not a substitute for clinical work. No chemical or pathological researches can do away with the necessity for an exact history of the case and a careful clinical examination.

To obtain an accurate history requires much patience and detailed questioning. The patient is apt to describe 
the symptoms of the present, and to forget the symptoms of the past. For example, a patient may state that his pain is almost constant; further cross-examination, however, may bring out the truth that at first the pain was not constant, but came on from two to three hours after food. In the later stages the constancy of the pain so impresses the patient, that the memory of the earlier sequence of events is almost obliterated.

Pain is usually a prominent symptom in gastric disease. The date of the first onset of the pain and its situation must be noted. The probability is that pain to the right of the middle line is duodenal or biliary, pain to the left, gastric. In the early stages the pain of any of these conditions may be referred to the epigastrium. Usually the pain of appendicular gastralgia is situated in the epigastrium, and may radiate to the right iliac region. Gall-bladder pain may radiate to the costal margin, right shoulder, or dorsal region. Radiation in gastric or duodenal ulcer is less common, and when present may indicate invasion of the pancreas. In perforated duodenal ulcer the patient may refer the pain to the right iliac fossa, but further questioning will elicit the fact that at first the pain was limited to the upper abdomen.

Inquiry should be made into the character of the pain. The pain of biliary colic may be agonising in its intensity; the pain of duodenal ulcer is rarely of such severity, although in some instances patients may be "doubled up " during an attack. In appendicular gastralgia often the patient speaks of severe discomfort rather than of pain.

The duration of the pain, its relation to food, and the extent to which it is influenced by drugs are important. Gall-bladder pain may be transient, or may last for many hours, and is uninfluenced by food or alkalis. Duodenal or gastric ulcer pain increases in intensity towards the time of the next meal, and is eased by taking more food, or by the 
administration of alkalis. The pain of carcinoma is less severe, and is less influenced by food or by alkalis. In gastric ulcer the patient is hungry but does not eat for fear of the pain, in carcinoma the patient does not eat because he has no appetite, and frequently he has marked aversion from all forms of meat. The pain of biliary colic is capricious in its onset, and follows no definite sequence. The attack commences without apparent cause. In duodenal or gastric ulcer often the patient can trace the onset of an attack to a sudden chill, to some indiscretion in diet, or to mental worry. The pain of appendicular gastralgia may be brought on by severe physical exercise. In duodenal ulcer the sequence of events is remarkably constant; the pain, coming on two or three hours after food, increasing in intensity, and relieved by food, often forms a clinical picture which cannot be mistaken.

In hypersecretion, whether due to duodenal or to gastric ulcer, or to appendicular disease, a very constant feature is the onset of pain in the early hours of the morning, waking the patient from sleep.

The kinds of food which cause pain should be inquired into; in gastric and duodenal ulcer the patient feels more comfortable on a milk diet, in appendicular gastralgia milk diet has not the same effect in lessening the pain. In gallbladder trouble change of diet has no effect, in gastroptosis and atony of the stomach fluids cause more discomfort than solids.

In appendicular gastra'gia the pain comes on either immediately or shortly after food. The same is true to a less extent in gastric ulcer towards the cardiac end of the stomach. As a rule, the longer the interval between the taking of food and the onset of the pain, the greater the probability of the ulcer being in the duodenum. The constancy, or otherwise, of the pain is often suggestive; pain, 
definitely related to food, coming on in attacks, with freedom from symptoms for weeks or months, generally spells duodenal ulcer. Similar attacks with discomfort and flatulence in the intervals may indicate duodenal ulcer or appendicular gastralgia. Irregular, short, severe attacks of pain, not influenced by food, suggest gall-bladder disease. Constant pain may accompany gastric cancer or hour-glass stomach. When such pain succeeds definite attacks with free intervals, it is indicative that stenosis, from ulcer, has ensued, or that an ulcer has invaded the peritoneum, or is invading neighbouring organs.

If there be a history of vomiting, its frequency, character, and amount should be noted. Copious vomiting, occurring every two or three days, of material containing articles of diet known to have been taken on previous days, indicates pyloric stenosis. The eructation or vomiting of a highly acid fluid without food remains, is symptomatic of hypersecretion.

The vomiting of bright blood in any quantity is due probably to gastric or to duodenal ulcer, to cirrhosis of the liver, or more rarely to aneurysm. The emesis of coffee ground material may be due to malignant disease or ulcer. The occurrence of "black stools" should be inquired into, and an attempt made to determine whether they were due to hæmorrhage or the taking of bismuth or other drugs.

The habits of the patient, especially with reference to over-indulgence in alcohol and cigarette smoking, may give a clue to the origin of the gastric trouble, while the possibility of syphilis must be borne in mind. A history of attacks of jaundice points to gall-bladder disease, either as a complication of gastric disorder, or as the cause of the gastric symptoms. 


\section{PHYSICAL EXAMINATION}

This includes both general and special examination.

A. General.-The general appearance of the patient should be noted. Severe anæmia in conjunction with gastric pain, in an otherwise healthy, well-nourished patient, suggests hæmorrhage from an ulcer. Anæmia with a worn-out look arouses suspicion of cancer. In cancer patients a diagnosis is often written on their faces. In duodenal ulcer the blood-pressure is often high, in carcinoma low, and the pulse feeble. The possibility that gastric pain may be due to the "gastric crises" of locomotor ataxy must not be forgotten, and the patient therefore should be examined for signs of this disease.

Kidney trouble, too, may simulate gastric disease, hence the need for a careful examination of the urine. A pelvic examination should be part of the routine, at any rate before deciding on any operative procedure.

B. Special.-Inspection of the abdomen may reveal a tumour, or an obviously dilated stomach. If there be visible waves of gastric peristalsis, pyloric stenosis is present. A dilated stomach without visible peristalsis may be due to gastric atony.

By palpation a pyloric tumour, or a swelling in the region of the gall-bladder, may be felt. A succussion splash felt more than three hours after food suggests gastric atony.

Palpation should be practised both before and after distension of the stomach with air by means of an inflating bellows.

In this way an otherwise impalpable tumour sometimes may be felt, and a tumour of the transverse colon or liver can be differentiated from a pyloric tumour.

Inflation of the stomach will reveal gastroptosis, as the displaced upper border of the stomach can be seen plainly, sometimes as low as the umbilicus. 
Auscultation is sometimes of service in the diagnosis of œsophageal stricture, or of cancer at the cardiac orifice of the stomach. Dr. Ogston has pointed out that on listening at a spot about three inches below the angle of the left scapula, an amphoric rushing sound is heard when the fluid enters the stomach. In œsophageal stricture the sound may be delayed until fourteen or sixteen seconds after the swallowing of food.

If stricture be suspected, an osophageal bougie should be passed, if necessary an anæsthetic being given to exclude spasm. Sometimes a subphrenic abscess containing gas may be diagnosed by means of auscultation.

Personally, I do not place much reliance on percussion or auscultatory percussion of the stomach, except in combination with distension of the viscus with air. The stomach limits mapped out by simple percussion rarely are confirmed on the operation table, or by X-ray examination.

\section{THE MOTOR FUNCTIONS OF THE STOMACH}

The simplest and most useful method of investigating the motor functions of the stomach is the following: The last thing at night, the stomach is washed out with warm water until the washings are returned clear. Usually about two pints of water are used. The quantity should be measured carefully, in order to ascertain whether all of it be recovered, or whether some of it be retained in the stomach. If a considerable amount of the water used be not recovered, it may be because it has passed into the distal compartment of an hour-glass stomach. After completion of the lavage, twelve ounces (340 c.c.) of milk is given to the patient. On the following morning, exactly ten hours later, the stomach tube is passed, and any fluid present in the stomach is withdrawn by aspiration with Senoran's apparatus (see Appendix, p. 290). Normally, 
under these conditions the stomach should be empty. Should there be any fluid withdrawn, it is measured, and the amount noted. It is then tested for acidity, and for the presence of bile and blood. The presence of a clear acid fluid without food residue indicates hypersecretion. The presence of food remains is a sign of pyloric stenosis, or motor insufficiency. It is a useful plan to give some raisins with the milk, as the presence of the skins and pips is recognised easily in the gastric contents.

In pyloric stenosis as much as 400 c.c. of fluid containing much food residue may be recovered from the stomach.

In duodenal ulcer, without stenosis, the motor power of the stomach is good, and no food remains are present in the early morning. Frequently in duodenal ulcer there is hypersecretion, so that from 30 c.c. to 50 c.c. of fluid may be withdrawn in the early morning. It is a clear or opalescent yellowish fluid, containing no food residue, and having an acid reaction. In appendicular gastralgia hypersecretion is a frequent, and in gastric ulcer an occasional symptom.

\section{EXAMINATION OF VOMIT}

When vomiting is one of the symptoms, the amount vomited at a time should be carefully measured. As a rule, in dilated stomach due to atony or pyloric stenosis, vomiting does, not occur more than once in the twenty-four hours, sometimes less frequently, but the quantity vomited may be enormous. In uncomplicated gastric or duodenal ulcer vomiting, when it occurs, usually does so an hour or two after meals, and consists of partially digested food. In pyloric stenosis the vomit has a very acid smell; in carcinoma it has often a very foul odour.

The vomit should be examined microscopically for blood and for bacilli, and also tested chemically for blood. Pus 
in the vomit may be due to the rupture into the stomach, of a perigastric abscess.

\section{GASTRIC ANALYSIS}

Personally, I attach great importance to the information to be gained from the examination of test-meals. I need hardly say that I do not maintain that a diagnosis should be based solely on a chemical examination, but if the results be interpreted in the light of the clinical history, careful examination of the motor activity, and of the secretions of the stomach, is a great help. In the following account I will attempt, in general terms, to indicate the variations found in different diseases, but it must be understood that the results are not absolutely constant. It is impossible to lay down hard and fast rules as to the relation of the different lesions found to the chemical alteration of the gastric contents. With experience, however, one is able to recognise the types of analytical results which usually are associated with particular lesions.

For purposes of gastric analysis a "test-meal" is given, and the contents of the stomach drawn off after a given interval. Pawlow has shown that the secretion of gastric juice varies according to the kind of food given, and according to the length of time digestion has progressed. Hence to make an accurate comparison of the analysis of one testmeal with that of another, the stomach must be empty at the time of the administration of the test-meal, the amount and composition of the meal must be the same, and the time which it is allowed to remain in the stomach must be the same. If these conditions be not complied with, the resulting analyses are of no value. Various test-meals are employed, but the following modification of that of Ewald is the simplest and most generally useful.

Usually the test-meal is given in the morning, half an hour 
after the investigation into the motor functions of the stomach (see p. 6) has been completed. The technique is as follows :

The test-meal I use consists of two slices of dry toast and fifteen ounces (430 c.c.) of weak tea with sugar, but no milk. The stomach contents should be drawn off exactly one hour after the patient has begun the meal.

The amount of gastric contents recovered should be measured carefully. In a normal stomach the amount recovered varies from 100 c.c. to 140 c.c. In pyloric stenosis the quantity recovered may be as much as 500 c.c. or 600 c.c. In duodenal ulcer the evacuation of the stomach is often quicker than normal, so that the amount of gastric contents recovered after a test-meal is less than in health. The colour, smell, and character of the contents are noted. The colour is usually a light brown; if much blood be present the colour is dark brown, the so-called " coffee grounds" appearance. Sometimes bright blood, recognisable as such, is present; in small quantity this may be due to trauma caused by the tube, in large quantity it may be from an open ulcer. Usually the smell of the gastric contents is not objectionable. If there be stagnation, the smell is very acrid, owing to the presence of butyric acid; in malignant disease in which there is extreme ulceration or sloughing of the growth, the smell may be extremely putrid. In normal digestion the toast is finely divided; in chronic gastritis, whether due to gastric ulcer or malignant disease, or secondary to appendix trouble, the gastric contents may be very thick and difficult to pour, and lumps of undigested toast, with much thick, ropy mucus, are present.

The gastric contents are then tested ${ }^{1}$ qualitatively and quantitatively.

1 An account of the various tests and methods of analysis will be found in the Appendix. 


\section{(a) Qualitative}

The qualitative tests are carried out on some of the gastric contents which have been filtered. The reaction should be acid. In severe chronic gastritis the reaction may be alkaline. The presence or absence of free hydrochloric acid is tested by Gunzberg's reagent or by the dimethyl-amido-azo-benzol test.

Free hydrochloric acid usually is present in duodenal ulcer, in gastric ulcer near the pylorus, and in gall-bladder disease, and sometimes in appendicular gastralgia.

It is absent as a rule in malignant disease, in severe chronic gastritis, in old gastric ulcer situated in the body or cardiac end of the stomach, in cases of hour-glass stomach, and in appendicular gastralgia.

The presence of bile pigment is tested for by Gmelin's test. Not frequently in duodenal ulcer there is regurgitation of bile in sufficient quantity to give the reaction.

Volatile acids usually can be detected by smell; the chemical test is as follows: Some of the unfiltered gastric contents are placed in a test-tube, a piece of blue litmus paper, moistened with distilled water, is stretched on the mouth of the tube, and the contents heated. If volatile acids be present, the blue paper is reddened owing to the liberation of volatile acids.

Lactic acid in small quantities is present almost invariably in the gastric contents obtained after an ordinary test-meal. Its presence is of clinical significance only if it be found after the administration of a special test-meal. The procedure devised by Boas is as follows :

The stomach is washed out overnight until the water returns absolutely clear. On the following morning the stomach is washed out again and a meal of oatmeal soup is given. The soup consists of one teaspoonful of rolled oats 
and 1000 c.c. ( 35 oz.) of water, boiled down to 500 c.c. $(17$ oz.), to which is added a little milk, but no salt. Two hours later the stomach-tube is passed, and the gastric contents drawn off. The contents are filtered, and tested for lactic acid with Uffelmann's reagent. Under these conditions lactic acid rarely is present except in gastric carcinoma. A positive test therefore is of great clinical significance, and points to cancer of the stomach.

\section{(b) Quantitative}

(i) Total Acidity.-In a healthy stomach the total acidity varies between 55 and 65 . If the total acidity be over 70 , a condition is present which may be termed hyper-acidity; if below 50, hypo-acidity.

Hyper-acidity must be distinguished clearly from hyperchlorhydria. The total acidity as estimated by Ewald's method represents the amount of total acids in 100 c.c. of gastric contents, i.e. the amount of hydrochloric acid; volatile acids, and of acid phosphates. Thus hyper-acidity may be due to excess of volatile acids, with normal or slight excess of hydrochloric acid; to increased free hydrochloric acid with normal or diminished volatile acids; or on the other hand, there may be hyperchlorhydria or excess of free hydrochloric acid, with only slight hyper-acidity.

The highest total acidity I have found is 170 , in a case of gastric ulcer near the pylorus, and the lowest 12 , in a case of cancer of the stomach.

In duodenal ulcer some degree of hyper-acidity is the rule (in 75 per cent. of the cases), hypo-acidity the exception, the average total acidity in my series of cases being 79 .

In gastric ulcer the amount of the total acidity varies considerably. In ulcer near the pylorus it is usually higher than is normal. In hour-glass stomach the total acidity is low. In gastric ulcer. in the body of the stomach or at the 
cardiac end, there is occasionally hyper-acidity, but more often hypo-acidity.

In malignant disease the total acidity almost invariably is low; only twice have I observed it higher than 50. The lowest total acidity I have met with is 12 . Usually, it varies between 25 and 40 , the average in my cases being 30 . In appendicular gastralgia the total acidity is generally slightly higher than normal. The average total acidity in my series of cases of appendicular gastralgia is 65 .

In diseases of the gall-bladder, as a rule, the total acidity is not markedly altered.

(ii) Volatile Acids.-Normally the amount of volatile acids as estimated by Harley's method varies from two to five. In appendicular gastralgia an appreciable increase in the amount of the volatile acids is the rule. On one occasion I found a percentage of $18 \cdot 0$. In malignant disease there usually is an increase. In one of my cases of carcinoma, confirmed by operation, there were no volatile acids present, but the growth was at the cardiac end of the stomach. In duodenal ulcer increase of the volatile acids is the exception, not the rule. In gastric ulcer, in my experience the volatile acids are not increased markedly unless there be associated appendicular disease.

(iii) The Total Chlorides. - In health the amount of the total chlorides varies from 0.310 to 0.330 , the average being about 0.320 .

In duodenal ulcer there is a very marked increase of the total chlorides; in one of my cases the amount was 0.514 , the average in my series being $0 \cdot 380$.

When pyloric obstruction exists, often there is a decrease. In gastric ulcer there is usually an increase, less marked, but to some degree varying with the site of the ulcer. When the ulcer is near the pylorus the increase is more marked than when it is situated at the cardiac end. 
When the ulcer, by cicatrisation, has produced pyloric stenosis or hour-glass stomach, commonly there is a decrease of the total chlorides.

In appendicular gastralgia the variation from the normal is not great, the tendency being towards a decrease.

(iv) Free Hydrochloric Acid.-In a healthy individual the amount of free hydrochloric acid in the gastric contents after a test-meal varies from 0.018 to 0.022 , the average being 0.020 .

In duodenal ulcer, at any rate in the earlier stage, hyperchlorhydria is the rule. When obstruction has supervened, or if the patient be examined between the attacks, especially if he have been on a purely milk diet, the free hydrochloric acid may be greatly diminished, or even absent. This, however, is the exception; in the majority of cases, the free hydrochloric acid is increased to a marked degree. In one case under my care the amount of free hydrochloric acid was $0 \cdot 127$ (verified by several analyses).

In the early stages of gastric ulcer free hydrochloric acid always is present in excess, but this does not hold good for the later stages. In active ulcers near the pylorus hyperchlorhydria is the rule. Thus in a case of gastric ulcer situated close to the pylorus, I found 0.072 of free hydrochloric acid.

When a gastric ulcer has caused pyloric stenosis the free hydrochloric acid is diminished or even absent. In ulcer of long standing situated in the body of the stomach, free hydrochloric acid is diminished or absent. Invariably, in my experience, in cases where cicatrisation has caused hourglass stomach, free hydrochloric acid is absent. In chronic gastritis free hydrochloric acid is diminished greatly, or is absent. In malignant disease free hydrochloric acid is absent almost invariably. I have met with only two instances of gastric carcinoma, verified by autopsy or by 
operation, in which free hydrochloric acid was present in the gastric contents.

In appendicular gastralgia the free hydrochloric acid is diminished markedly or absent altogether, but very occasionally there is hyperchlorhydria.

(v) The Protein Hydrochloric Acid.-As a rule the amount of protein hydrochloric acid varies from 0.220 to 0.250 . The importance of estimating the protein hydrochloric acid lies in the circumstance, that in malignant disease the amount of protein hydrochloric acid is diminished to marked degree. This is a most valuable aid in the diagnosis. In only two cases of carcinoma have I found the amount of protein hydrochloric acid to be over 0.200 , and usually it is less than $0 \cdot 100$.

In chronic gastritis the protein hydrochloric acid is diminished. In other gastric conditions the variations are not of clinical significance.

(vi) The Mineral Chlorides.-In malignant disease there is almost constantly a marked increase in the mineral chlorides. As the total chlorides are diminished, it is probable that some of the hydrochloric acid is neutralised by an alkaline fluid secreted by the growth and so converted into fixed chloride.

The following are some typical gastric analyses. In all the cases to which they refer, a correct diagnosis was made and verified by operation :

\begin{tabular}{|c|c|c|c|c|c|}
\hline Lesion. & $\begin{array}{l}\text { Total } \\
\text { Acidity. }\end{array}$ & $\begin{array}{c}\text { Total } \\
\text { Chlorides. }\end{array}$ & Free $\mathrm{HCl}$. & $\begin{array}{c}\text { Protein } \\
\text { HCl. . }\end{array}$ & $\begin{array}{c}\text { Mineral } \\
\text { Chlorides. }\end{array}$ \\
\hline Duodenal ulcer & 82 & 0.350 & 0.038 & $0 \cdot 229$ & 0.800 \\
\hline,$\quad \quad$, & 80 & $0 \cdot 405$ & 0.055 & $0 \cdot 275$ & 0.075 \\
\hline Gastric ulcer (close to pylorus) & 75 & $0 \cdot 325$ & 0.040 & 0.205 & 0.800 \\
\hline ", (about middde) & 78 & $0 \cdot 308$ & 0.025 & 0206 & 0.077 \\
\hline ", (hour-glass stomach) & 70 & $0 \cdot 332$ & 0.000 & $0 \cdot 230$ & 0.098 \\
\hline Carcinoma & 28 & $0 \cdot 178$ & 0.000 & 0.051 & $0 \cdot 124$ \\
\hline Appendicular gastralgia & 61 & 0.288 & 0.007 & 0.201 & 0.080 \\
\hline
\end{tabular}




\section{X-RAYS}

To examine the stomach by means of the $\mathrm{X}$-rays, a meal is administered consisting of two ounces of bismuth oxychloride mixed with bread and milk or with porridge. The examination should be made both in the vertical and in the horizontal positions. The presence of such foreign bodies, as are opaque to the $\mathrm{X}$-rays, is detected at once by examination with the screen.

The existence of an œesophageal stricture is ascertained readily, the obstruction to the onward passage of the food and the dilatation of the osophagus above the stricture, being clearly visible. The size, shape, and position of the stomach can be determined with fair accuracy. Hour-glass stomach can be recognised by means of the X-rays, but care must be taken not to mistake a functional contraction. In organic hour-glass stomach it will be seen that the constriction does not pass from the most dependent part of the proximal compartment, as is the case in the functional condition, and further, the spasmodic contraction will disappear on vigorous massaging of the abdomen.

Spasm near the middle of the stomach is often a sign of gastric ulcer. In a dilated stomach due to pyloric stenosis, vigorous waves of peristalsis are seen, but yet very little or no food is seen to pass into the duodenum.

Occasionally a reverse peristalsis is visible. In dilated stomach due to atony, the feebleness of the waves, and the sinking of the food to the most dependent part of the stomach, indicate the cause of the dilatation.

In cases of duodenal ulcer, without obstruction, the stomach often is seen to be actively contracted (hypertonic). When examined with the $\mathrm{X}$-rays in the erect position, it is apparent that the greater curvature does not reach to the umbilicus. In gastric ulcer hypertonus rarely is present. 


\section{GASTROSCOPE}

At present the gastroscope is not of great assistance in the diagnosis of gastric disease. An ulcer may be seen, but it is impossible to differentiate between a simple lesion and a malignant one. The best instrument is that designed by Dr. William Hill.

\section{EXPLORATORY INCISION}

When all other means of diagnosis have failed, abdominal exploration may be indicated. This rarely is necessary except in cases of suspected cancer. In the great majority of cases a diagnosis of the necessity for surgical treatment can be made, but if cancer of the stomach is to be dealt with surgically, with good result, the abdomen must be opened, in some cases, without a definite diagnosis having been made. 


\section{CHAPTER II}

\section{THE PREPARATION AND TREATMENT OF PATIENTS BEFORE GASTRIC OPERATIONS}

LIKE success in other walks of life, success in gastric surgery depends on attention to detail. There are some who scoff at elaborate preparations, minimise the importance of aftertreatment, and regard the patient's fate as sealed for good or ill when the wound is closed. I think this is a mistake. Attention to detail in the treatment of patients before and after operation, is second only to the skilful performance of the operation itself. Often skilful nursing and after-treatment will pull through a seemingly hopeless case, and always will add greatly to the patient's comfort and prospect of a speedy convalescence. There is no finality in surgery. Methods are being changed continually. I do not propose to describe, or even to summarise, all the different methods in use, but simply to give an account of those, which from an experience of over a thousand operations, as well as from watching the practice of many other surgeons in this country, America, and on the Continent, personally I have found to give the best results, and which from experience I can recommend.

\section{PREPARATORY TREATMENT}

The minimum period of preparation for an abdominal operation (except, of course, in case of emergency) should be three days, but whenever practicable I prefer the preparation of the patient to be commenced a week before the date fixed for the operation. During this period the bowels are 
well opened by several doses of calomel, the last dose being given two nights previous to the operation. On the morning of the day before operation a dose of castor oil is given. The doses of these drugs must be regulated according to the age and strength of the patient, so as to secure thorough emptying of the bowels without purging. Diarrhœa is a bad preparation for an abdominal section. Enemata may be necessary as well as calomel, and in any case a plain soap-and-water enema should be given on the morning of the operation. It is a good plan for the patient to try sleeping in the propped-up, semi-sitting position, otherwise this may be found irksome after the operation. Likewise the patient should be encouraged to practise taking deep breaths at intervals during the day. This will tend to minimise the danger of hypostatic congestion of the lungs after the operation. I think, too, that such preparations give the patient something to think about, and give confidence by encouraging the belief that everything possible is being done to ensure a successful operation. During the whole period of preparation the patient is kept on a fluid diet, and for at least fifty hours nothing but sterilised milk and tea is allowed. By this means it is possible, as Professor Harvey Cushing has shown, to render sterile, the upper part at least, of the alimentary tract.

Especial care should be given to the condition of the mouth. The teeth should be brushed three times daily with carbolic tooth powder, and the mouth rinsed frequently with listerine or hydrogen peroxide (6 volume solution). Stumps or badly-decayed teeth should be extracted.

I have no doubt that some cases of sepsis after abdominal operations are due to a septic state of the mouth. Cleansing the mouth, and emptying of the intestinal canal, greatly reduce the risk of infection from within. The patient should remain in bed for two days previous to the operation. 
Preparation of the Operation Area.-The skin of the abdomen is prepared in the following way: Two nights previous to the operation the pubes is shaved, and the patient takes a hot bath. If the patient be too ill for a bath, the abdomen is washed over with acetone. On the night previous to the operation the whole abdomen is well rubbed over for five minutes with acetone, and then swabbed over with iodine paint. This paint is made up as follows :-

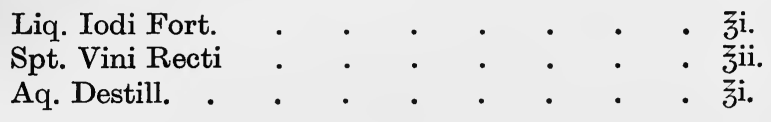

Then the whole abdomen is covered with a dry sterilised sheet of gauze or lint, which is kept in place by a sterilised bandage. As soon as the patient is placed on the operation table the bandage is removed, and while the patient is being anæsthetised the whole abdomen is painted over again with iodine paint. When anæsthetisation is complete, the superfluous iodine is wiped off with a piece of sterile gauze. After an extensive trial of many other methods of skin preparation, I have found that the one above described is not only the simplest, but the most reliable. It takes little time, there is no necessity (except in exceptional cases) to shave the whole operation area, and the long exposure necessitated by most other methods is avoided. The skin tested bacteriologically is invariably sterile. In a number of cases I have snipped off a fragment of skin at the conclusion of the operation and placed it in a culture tube, and in one case only has a growth of micro-organisms resulted on incubation. Further-and this to my mind is a great advantage of this method-its rapidity and reliability render it equally applicable for emergency operations. Simplification and uniformity of methods form one of the ideals to be aimed at in all surgical procedures.

In cases of pyloric and intestinal obstruction the stomach 
should be washed out two or three hours before the operation. In elderly and feeble patients it is a good plan to give a pint of saline solution per rectum a couple of hours before the operation. As an additional precaution in such cases, while the patient is being anæsthetised, a needle may be inserted in the axilla, and a pint of saline solution run into it from a rubber bag, as practised by Mr. Arbuthnot Lane.

During a visit to the chief clinics of the United States in the year 1907, I was impressed greatly with the method of open administration of ether employed so extensively in that country, and since then have had scarcely any other anæsthetic given to my patients, and I am convinced, contrary to my former opinion, that ether is a far more satisfactory anæsthetic than chloroform. Ether is undoubtedly safer, and is not less pleasant than chloroform; it is followed very rarely by post-anæsthetic vomiting, and is less depressing than chloroform. With ether the signs of shock are much less marked than with chloroform.

If the patient is to have ether by the open method, a hypodermic injection of morphia, gr. $\frac{1}{6}$, and atropine sulph. gr. $\frac{1}{10 \sigma}$, should be given half an hour before the time fixed for the operation.

The previous administration of morphia composes the patient before operation, diminishes the amount of ether requisite to induce and maintain anæsthesia, and delays the return to consciousness of pain. The atropine checks troublesome secretion of mucus in the trachea and bronchi, and lessens the sweating so common when ether is given alone. 


\section{CHAPTER III}

\section{OPERATIONS ON THE STOMACH}

The Incision.-It is best to open the abdomen just to the right of the middle line. A gastro-jejunostomy can be performed through an incision $2 \frac{1}{2}$ inches in length, but such an incision does not suffice for an adequate examination of the gall-bladder and appendix. No operation on the stomach should be performed until the whole of the viscus has been examined, and the surgeon has satisfied himself as to the lesion present. Without adequate exposure it is easy to overlook an hour-glass stomach, and to mistake the distal pouch for the whole of the viscus. If, as has been done in some of the recorded cases, a gastro-jejunostomy be performed on the distal pouch, the patient will gain no relief. It is absolutely essential, therefore, before proceeding with any operative measure, to make a very careful examination of the stomach and other viscera. The time so spent is never wasted. In every case the appendix and gall-bladder should be examined, and dealt with, if necessary, and if the patient's condition permit. After incising the skin and fascia, the sheath of the right rectus is divided just to the right of the middle line. The rectus muscle is displaced outwards, and the posterior sheath and peritoneum divided between two pairs of forceps, in the line of the incision through the anterior sheath.

Closure of the Wound.-The closure of the wound is effected as follows: A large curved needle armed with a double thread of No. 2 iodised catgut is used. The suture 
is commenced at the lower end of the wound, and after the first suture has been tied, the needle is passed from the inner surface of the peritoneum, so as to turn the cut edge of the peritoneum outward, as suggested by Dr. J. B. Murphy. The suture includes the transversalis fascia and posterior sheath of the rectus. It is continued without interruption to the upper end of the wound, where the needle is passed under the last loop and drawn tight. The needle is laid aside without cutting the catgut. I have all my needles made with a special " grip-eye" designed to prevent accidental unthreading of the suture material (Fig. 1). A

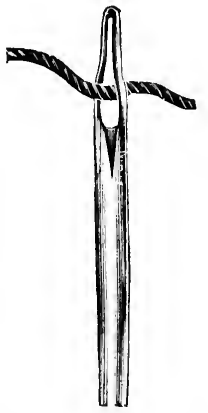
few silkworm-gut sutures are now passed through the remaining structures of the abdominal wall, viz. through the skin, anterior sheath of the rectus, the fibres of the recti muscles, and anterior sheath and skin of the opposite side. These sutures are passed at intervals of an inch, so that in a wound four inches long, three interrupted sutures are employed. The ends of these sutures are FIG. 1.-The author's secured temporarily with pressure forceps. "grip-eyed" needle. The anterior sheath of the rectus is now sutured with the same needle and suture as was used for the peritoneum. The suture is continued from the upper angle of the wound to the lower, where it is tied to the end which was left long for the purpose when commencing the suturing. The ends are then cut short.

The skin incision is closed by a sub-cuticular suture of silkworm-gut. The operation area is now painted over with iodine paint, and covered with silver leaf. A folded strip of gauze about an inch wide is laid lengthwise on the wound, and the interrupted sutures are tied over it. This anchors the dressing, and at the same time prevents the discomfort caused by the sutures cutting into the skin. 
The important points in the technique of abdominal wounds are:

1. Perfect hæmostasis.

2. Avoidance of tearing of muscle or fascia.

3. Obliteration of "dead spaces."

4. The protection of the edges of the wound from bruising and contamination.

5. Accurate apposition of each layer.

\section{GASTROTOMY}

Indications :

(i) For the removal of foreign bodies from the stomach or lower end of the œesophagus.

(ii) For the removal of growths from the interior of the stomach.

(iii) For dilating the pylorus (Loreta's operation).

(iv) In rare instances for gastric hæmorrhage.

\section{(i) For the Removal of Foreign Bodies}

(a) From the Stomach.-All kinds of foreign bodies have been found in the stomach : hairballs, nails, false teeth, pins, stones, coins, and many other articles. Often they cause no symptoms, and in some cases have remained in the stomach for years without causing trouble.

After opening the abdomen the stomach is packed round with pads so as to prevent contamination of the peritoneal cavity with gastric contents. The stomach is drawn partially out of the abdomen and a vertical incision about two inches in length is made into the viscus. The foreign body is removed by a suitable pair of forceps. In the case of a small foreign body it is a help to pass a hand up behind the transverse colon to the posterior aspect of the stomach, and to steady the foreign body while it is being grasped by the forceps. The wound in the stomach is then sewn up with 
two continuous sutures of linen thread, an inner suture including all coats of the viscus, and an outer serous suture. The abdominal incision is then closed, as described already, without drainage.

(b) From the Lower End of the Esophagus.-Occasionally a foreign body lodges in the lower end of the œsophagus and neither can be drawn upwards, nor pushed downwards into the stomach. In such cases the foreign body usually can be removed by forceps introduced through a gastrotomy opening. Some years ago I saw a gentleman who, in a fit of melancholia, had swallowed a metal case $3 \frac{1}{2}$ inches long containing a glass creasote inhaler. After localisation with the X-rays, I opened the stomach and withdrew the case by means of a pair of long forceps, without difficulty.

Dr. Bull removed successfully a peach stone by attaching a sponge to a piece of string and dragging the sponge upwards, from a gastrotomy opening, to the mouth.

Gastrotomy for the removal of foreign bodies is a very safe operation, the mortality rate under modern conditions being well under 2 per cent.

(ii) For the Removal of Growths from the Interior of the Stomach

In these cases, as more room is required, it is advantageous to make the incision into the stomach in the direction of its long axis. After removal of the growth and the arrest of all hæmorrhage, the stomach is closed as usual by two continuous sutures.

(iii) For Dilating the Pylorus (Loreta's Operation)

This operation seldom is performed nowadays. Gastrojejunostomy is safer, although Mr. Burghard has had very good results in the treatment of infantile pyloric stenosis by means of dilatation. After opening the stomach, a pair of dilating 
forceps is introduced into the pylorus, which is then stretched as much as is possible without tearing the peritoneal coat.

\section{(iv) For Gastric Hæmorrhage}

For gastric hæmorrhage, the incision into the stomach is made in the direction of its long axis, and the source of the hæmorrhage sought for, an electro-cystoscope being used to illuminate the interior of the organ. The posterior wall of the stomach may be examined by invaginating it, and pushing it forwards through the incision in the anterior gastric wall.

Personally, I am not in favour of operations for gastric hæmorrhage during the progress of bleeding. The mortality from such operations is very high, and in my opinion it is safer to trust to medicinal means for the temporary arrest of the hæmorrhage, and then to perform gastro-jejunostomy later on, if it be considered advisable.

\section{GASTROSTOMY}

Indications :

(i) Malignant stricture of the œsophagus.

(ii) Impermeable fibrous stricture of the œsophagus.

Many different methods of performing gastrostomy have been devised. In my experience that of Senn is the best, and it is the one I now employ invariably. It is performed as follows :

A vertical skin incision $2 \frac{1}{2}$ inches long is made over the outer third of the left rectus, commencing one inch below the costal margin. The anterior sheath of the rectus is incised and fibres of the muscle separated, care being taken not to injure any nerves. The peritoneum is then opened, and the stomach sought by two fingers introduced into the peritoneal cavity. I have seen the colon opened in 
error for the stomach, but it is a mistake which should not occur.

The stomach is drawn gently into the wound, and a spot at the cardiac end, midway between the lesser and greater curvatures, is selected for the site of the opening. . A small incision is made, large enough to admit a No. 12 rubber catheter, and the catheter is introduced into the cavity of the stomach for a distance of two inches, and secured by a single catgut stitch. Three (or four if sufficient stomach be available) purse-string sutures are inserted round the tube. The first suture is passed so that it surrounds the tube in the form of a circle, with its circumference at a distance of half an inch from the tube. When the suture has been inserted, the catheter is depressed by the assistant towards the cavity of the stomach, and the suture tied sufficiently tightly to grip, but not to constrict, the catheter. A second circular purse-string suture is then inserted at a distance of one quarter of an inch from the first. The catheter is again pushed inwards and the suture tightened, tied, and cut short. A third, and in some cases a fourth, suture is passed in a similar manner. When all the sutures have been inserted and tied, the result is that a cone of the stomach is inverted into the cavity of the organ, with the catheter projecting at its apex. The stomach is fixed to the parietal peritoneum by two sutures of silkworm-gut, passed through all layers of the abdominal wall and through the peritoneal and muscular coats of the stomach, at a distance of half an inch above and below the tube. The abdominal incision is closed as already described.

At the conclusion of the operation a glass funnel is attached to the catheter by means of a piece of rubber tubing with a glass connection, and half a pint of warm milk or meat essence is introduced into the stomach. The catgut securing the catheter is digested usually in nine or ten 


\section{Plate I.-GASTROSTOMY.}

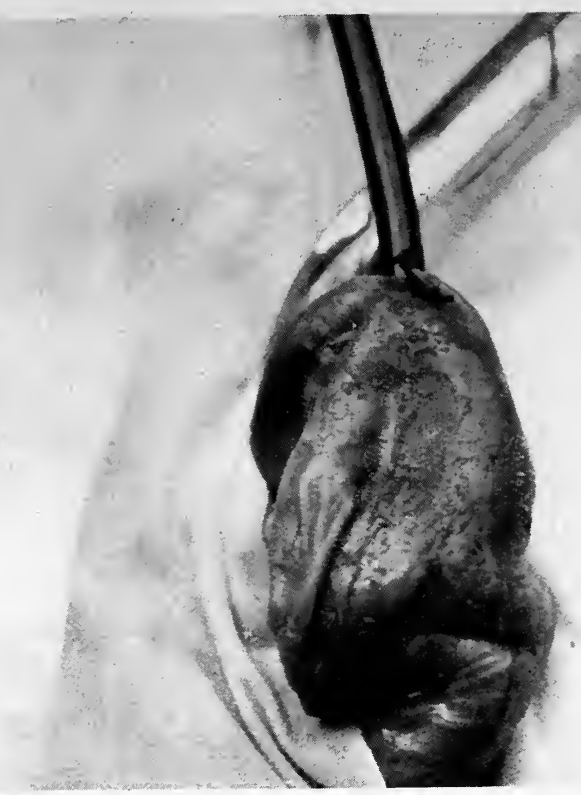

FIG. 2.-The stomach has been opened, and the catheter fixed in the opening by a catgut stitch.

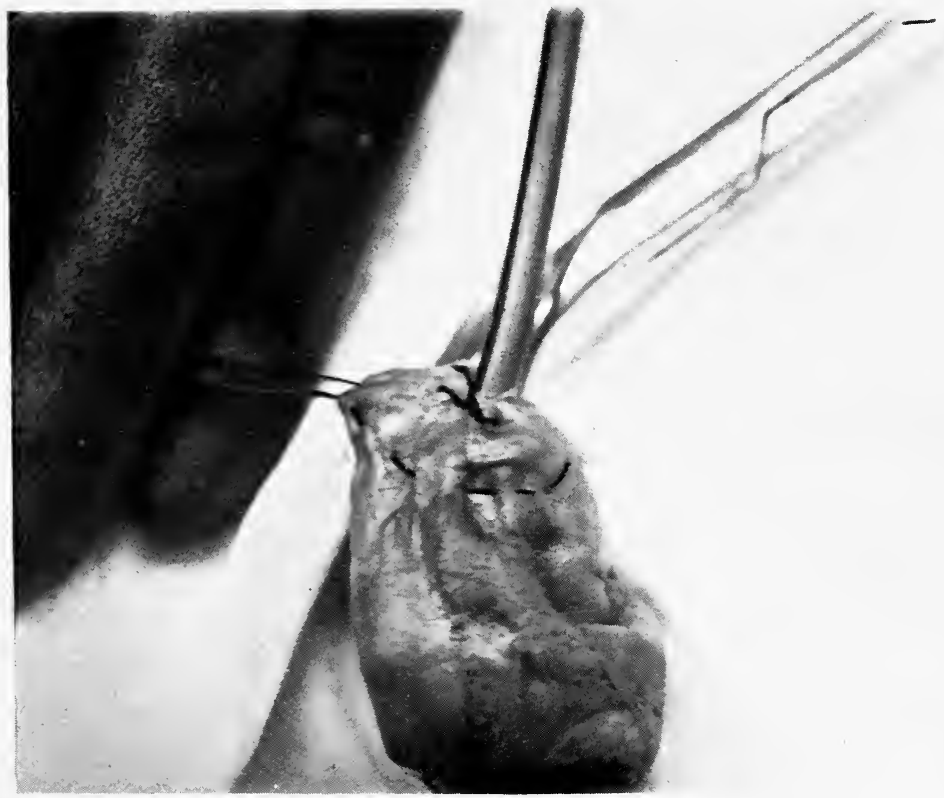

FIG. 3.-The first purse-string suture has been inserted and tied. The second purse-string suture is seen ready to be tightened. 
Plate II.-GaStRostomy.

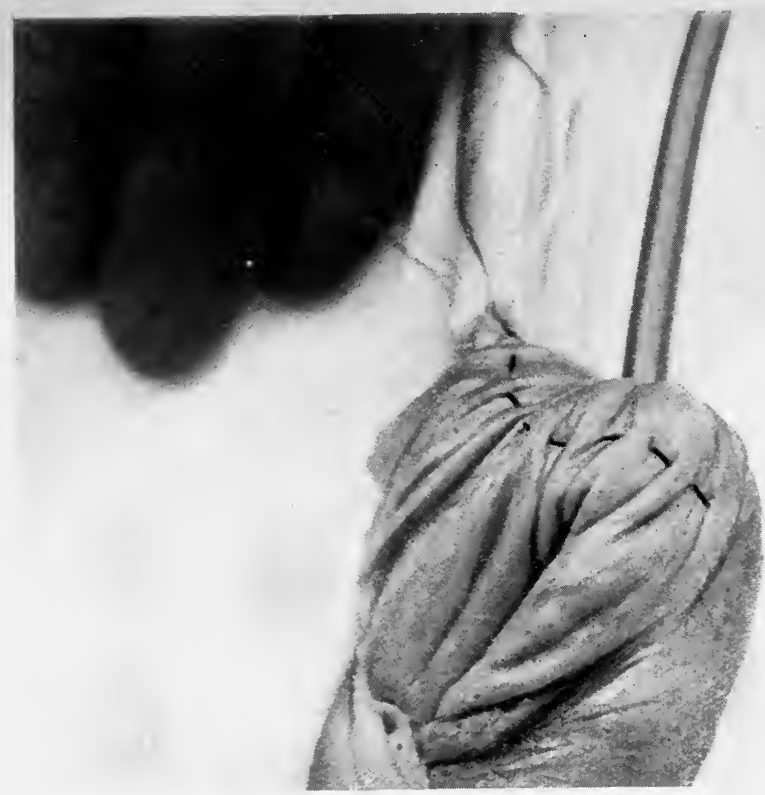

FIG. 4. - The second purse-string suture has been tightened, and the third is seen inserted and ready to be tightened.

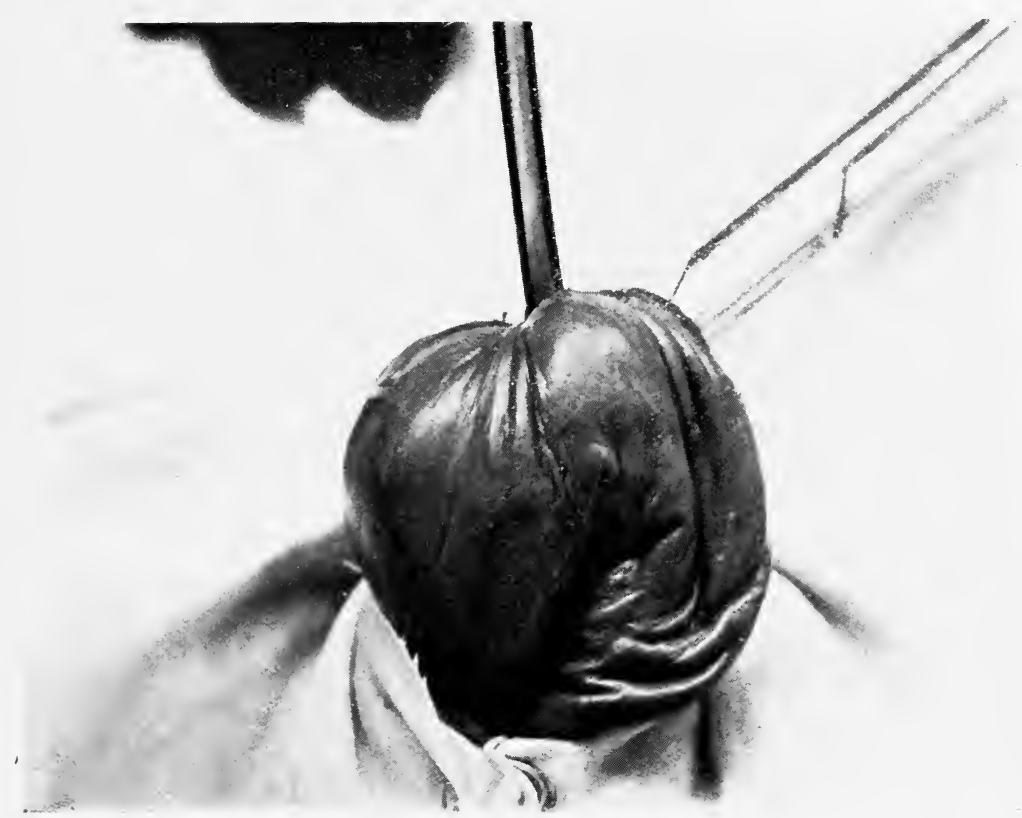

FIG. 5.-The four purse-string sutures have been inserted and tightened, and the invagination has been completed. 


$$
\text { , }
$$


days, and the catheter comes out. It is then introduced when required for feeding purposes. Senn's method is very satisfactory. If the operation be performed properly, leakage never occurs, and the patient's condition is very different from that obtaining after operations by the older methods, after which leakage of gastric juice produces such excoriation of the surrounding skin that life is scarcely worth living.

\section{PYLOROPLASTY}

The operation of pyloroplasty for pyloric stenosis consists in making an incision three inches in length through the pylorus in its longitudinal axis, and then suturing the incision in a vertical direction, thus increasing the diameter of the pyloric lumen. The operation, although in principle good, is unsatisfactory in practice. The after-results are disappointing.

In my Hunterian lectures I pointed out that relapse ensues in at least 30 per cent. of the cases in which the operation has been performed. An operation which cannot show better after-results than this cannot be compared with gastro-jejunostomy. It is seldom performed in the present day.

\section{PYLORO-GASTRO-DUODENOSTOMY (FINNEY'S OPERATION)}

In 1902 Dr. Finney described an operation which he called a " new method of pyloroplasty," but which, strictly speaking, is a gastro-duodenostomy. It is an improvement on pyloroplasty, but whether it is an efficient alternative to gastro-jejunostomy is doubtful.

The immediate mortality, except in the hands of its inventor, is higher than that of gastro-jejunostomy, and the after-results, so far as can be determined, are hardly so satisfactory. 
Dr. W. J. Mayo writes: "The Finney operation gave remarkably good ultimate results in pyloric obstruction, but in cases of unhealed ulcer existing proximal to or distal from the parts involved in the operation, less benefit was derived, unless the ulcer lay within the zone of the operative field, so that it could be coincidentally excised."

Dr. Finney's description of his operation is as follows : "Divide the adhesions binding the pylorus to the neighbouring structures; also free as thoroughly as possible the pyloric end of the stomach, and the first portion of the duodenum. Upon the thoroughness with which the pylorus, lower end of the stomach, and upper end of the duodenum are freed depends in a large measure the success of the operation, and the ease and rapidity of its performance. I wish to emphasize this as one of the most important points in the operation. Frequently, at first sight the pylorus may seem hopelessly bound down, when after a little patient toil and judicious use of the scalpel and blunt dissector it is found that it can be freed with comparative ease. A suture, to be used as a retractor, is taken in the upper wall of the pylorus, which is then retracted upwards. A second suture is then inserted into the anterior wall of the stomach, and a third into the anterior wall of the duodenum, at equidistant points-say about twelve centimetres from the suture just described in the pylorus. These second sutures mark the lower ends of the gastric and duodenal incisions respectively. They should be placed as low as possible, in order that the new pylorus may be amply large. Traction is then made upwards on the pyloric suture, and downwards in the same plane on the gastric and duodenal sutures. This keeps the stomach and duodenal walls taut, and allows the placing of the sutures with greater facility than if the walls remained lax. The peritoneal surfaces of the duodenum and stomach along its greater curvature are then sutured together as far 
posteriorly as possible. For this row I would recommend the use of the continuous suture, as it is more easily and quickly applied, and it can be reinforced after the stomach and duodenum have been incised. After the posterior line of sutures has been placed, an anterior row of mattress sutures is taken, which are not tied, but left long, in the manner indicated. These sutures, after they have been placed, are retracted vertically in either direction from the middle of the portion included in the row of sutures. Then, after all the stitches have been placed and retracted, the incision is made in the shape of a horse-shoe. The sutures should be placed far enough apart to give ample room for the incision. The gastric arm of the incision is made through the stomach wall just inside the lowest point of the line of sutures, and is carried up to and through the pylorus, and around into the duodenum, down to the corresponding point on the duodenal side. Hæmorrhage is then stopped. It is well to excise as much as possible of the scar tissue upon either side of the incision, in order to limit, as far as possible, the subsequent contraction of the cicatrix. This procedure I carried out in two of my cases with great satisfaction, and I should strongly recommend it in all cases where the walls of the pylorus are much thickened and there is much scar tissue present. It is well, too, to trim off with scissors redundant edges of mucous membrane, in order to prevent the formation of a valve-like fold of mucous membrane at the new pylorus. A continuous catgut suture is now taken through and through all coats of the intestine on the posterior side of the incision. This reinforces the posterior line of sutures, secures better approximation of the cut edges of the mucous membrane, and prevents the reunion of the divided intestinal walls. The anterior sutures are then straightened out and tied, and the operation is complete, unless one wishes to reinforce the mattress sutures with a few Lambert stitches. This pro- 
cedure, as is readily seen, gives the minimum of exposure of infected surface. All the stitches are placed, and the posterior row tied, before the bowel is opened, and it remains open just long enough to control the hæmorrhage. The size of the newly-formed pyloric opening is limited in this operation only by the mobility of the stomach and duodenum and the judgment of the operator. In all of my cases the incision has been about twelve centimetres in length, and could have been made longer had I chosen to make it so. Unless the stomach is very much dilated, or has descended to an unusual extent, the lower limit of the new outlet is at or near the level of its most dependent portion."

\section{GASTRO-JEJUNOSTOMY}

The operation of gastro-jejunostomy consists in making an anastomotic opening between the stomach and upper part of the jejunum. The communication may be made on the anterior or on the posterior wall of the stomach. Nowadays the posterior operation is performed almost universally. Although usually I perform the posterior operation, I am of opinion that it is immaterial which method be adopted. My experience is that either operation, in suitable cases, gives excellent results. I think that perhaps there is a slightly greater tendency to vomiting during the first few days after the anterior operation. The patients on whom the posterior operation is performed seem a little more comfortable at first, and convalesce a little more rapidly. As to the ultimate results there appears to me to be no difference. The only objection to the anterior operation is the possibility, that it is more apt to be followed by a jejunal ulcer than the posterior no-loop operation. But this is not certain. I will describe both methods in detail.

Anterior Gastro-jejunostomy.-After opening the peritoneal cavity the stomach and transverse colon are drawn 
Plate III.-ANTERIOR GASTRO-JEJUNOSTOMY.

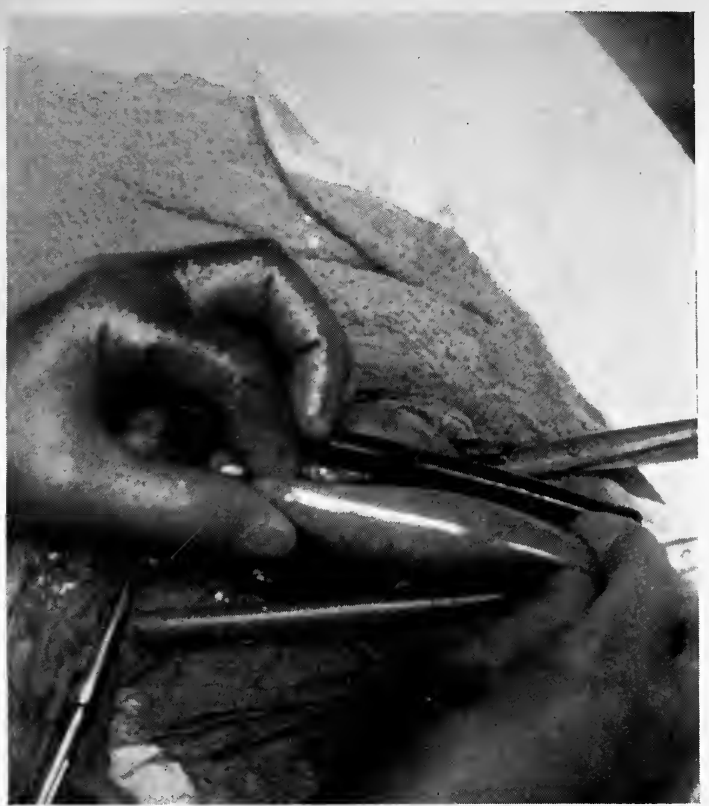

FIG. 6. - The jejunum is held by the operator while the assistant applies the clamps.

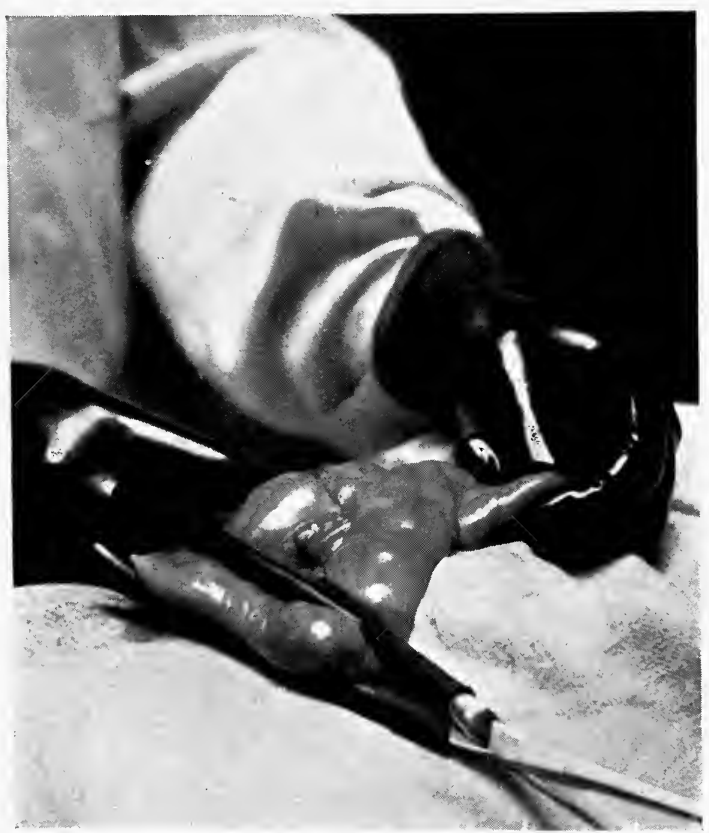

FIG. 7.-The jejunum clamped eight inches from its origin, which is grasped by the fingers of the left hand.

[For the photographs of gastro-jejunostomy I am indebted to Dr. Howard Kelly. They were taken by him during two of my operations while he was on a visit to this country two years ago.] 
PLATE IV. -ANTERIOR GASTRO-JEJUNOSTOMY.

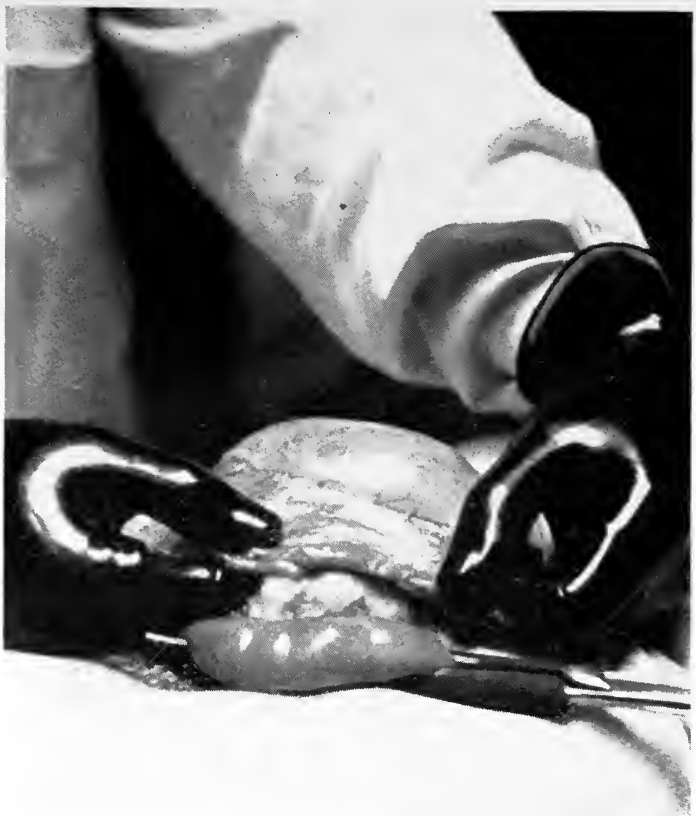

FIG. 8. - The transverse colon is drawn downwards, so as to make sure that the loop of jejunum can be applied to the stomach without tension.

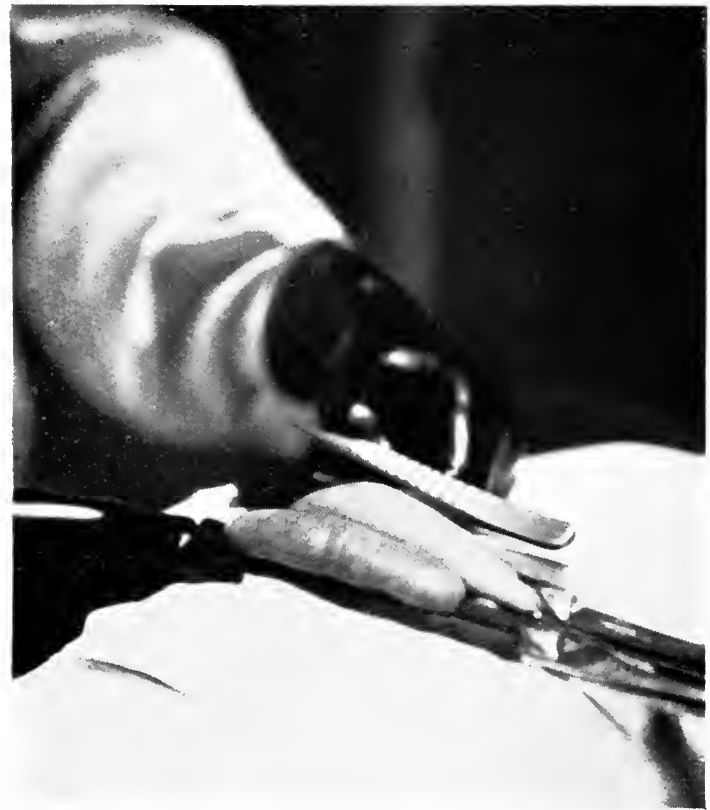

FiG. 9.- The clamped portions of viscera rearly for suturing. The abdominal cavity is shut off by pads. 
gently upwards, and two fingers introduced into the abdomen seek the commencement of the jejunum close to the left of the spine at the root of the transverse meso-colon.

Having made certain of the origin of the jejunum, it is traced downwards, and a portion is selected which can be applied to the anterior wall of the stomach without constricting the transverse colon. The selected portion of the jejunum is then clamped in a long pair of forceps, the blades of which are sheathed in rubber tubing (Plate III, Fig. 6). The portion clamped should be at least four inches in length, and is usually from six to eight inches from the duodenojejunal junction, the distance depending on the extent of the great omentum.

(Plate III, Fig. 7.) The intestine has been clamped. The origin of the jejunum is shown grasped by the fingers of the left hand.

The transverse colon is drawn downwards (Plate IV, Fig. 8) in order to make sure that the clamped portion of the jejunum can be applied to the stomach without tension.

A portion of the anterior wall of the stomach, at least four inches in length, is clamped in a second pair of forceps. The portion clamped should be as close to the pylorus as possible, and near to the lower border of the stomach.

Pads are tucked between the clamps and on each side of them, so that no viscera are visible in the field of operation, except the required portions of stomach and jejunum.

The handles of the forceps are held side by side by the assistant, thus bringing the clamped portions of stomach and jejunum side by side (Plate IV, Fig. 9), the upper end of the clamped jejunum being on the patient's left side, and the lower end to the right. The anastomosis is thus isoperistaltic.

A guide suture is inserted at each end of the clamped viscera (Plate V, Fig. 10). These sutures are four inches 
apart, and their object is to ensure that the line of suturing shall be straight.

The anastomosis is now effected by means of two continuous sutures, an inner hæmostatic suture penetrating all the coats of the viscera, and an outer approximating suture including the peritoneal, muscular, and submucous coats. For both sutures I use a very fine black Irish linen thread (introduced by Mr. H. J. Stiles). The outer serous suture is commenced at the left end of the clamped portions of stomach and jejunum, and is continued towards the right for four inches (Plate V, Fig. 11). Without being unthreaded, the needle is clamped with a pair of pressure forceps and laid upon the muslin sheet above the wound, ready for use later. A portion of the stomach as close to the suture line as possible is picked up with a pair of dissecting forceps, and the stomach is opened by cutting into it with a pair of small scissors (Plate VI, Fig. 12). The incision is then prolonged in both directions until the opening measures three inches. I attach great importance to making the anastomotic opening of sufficient size. I have a mark on the handle of my scalpel $2 \frac{1}{2}$ inches from its extremity, and by using this as a measure, I make sure that the opening is at least $2 \frac{1}{2}$ inches in length.

A similar opening is made in the jejunum. As the viscera are opened the mucous membrane pouts into the wound. With a pair of scissors, about a quarter of an inch of the mucous membrane is removed on each side of the opening into the stomach. As a rule I do not remove any of the jejunal mucosa.

The inner or hæmostatic suture, penetrating all coats of the stomach and jejunum, is now introduced. The posterior cut edges of the walls of the stomach and jejunum are held in apposition by means of two pairs of Porrier's forceps, one pair being applied at each end of the opening (Plate VI, 
Plate V.-ANTERIOR GASTRO-JEJUNOSTOMY.

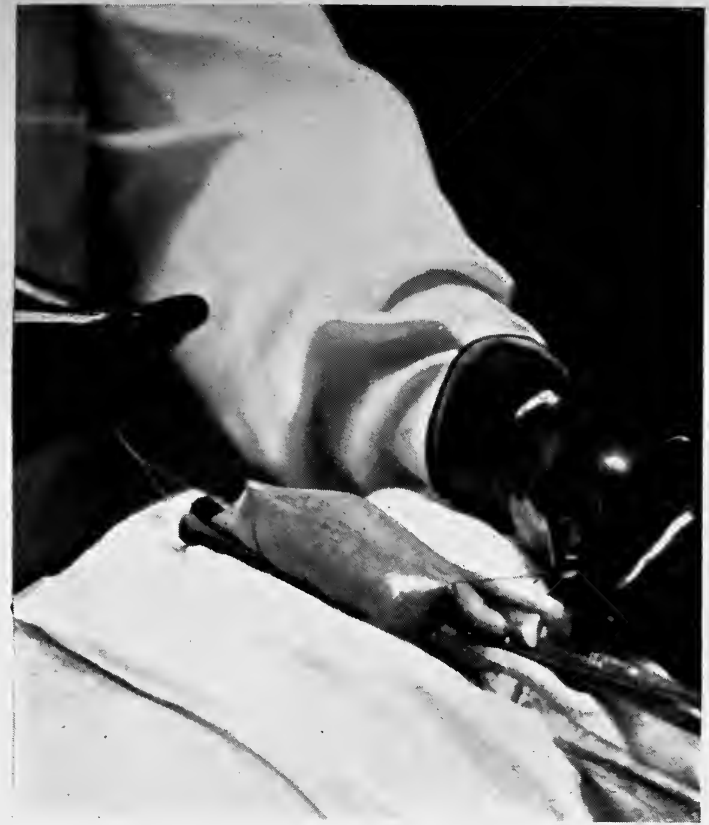

FIG. 10.-The stomach and jejunum united at each end by a guide suture.

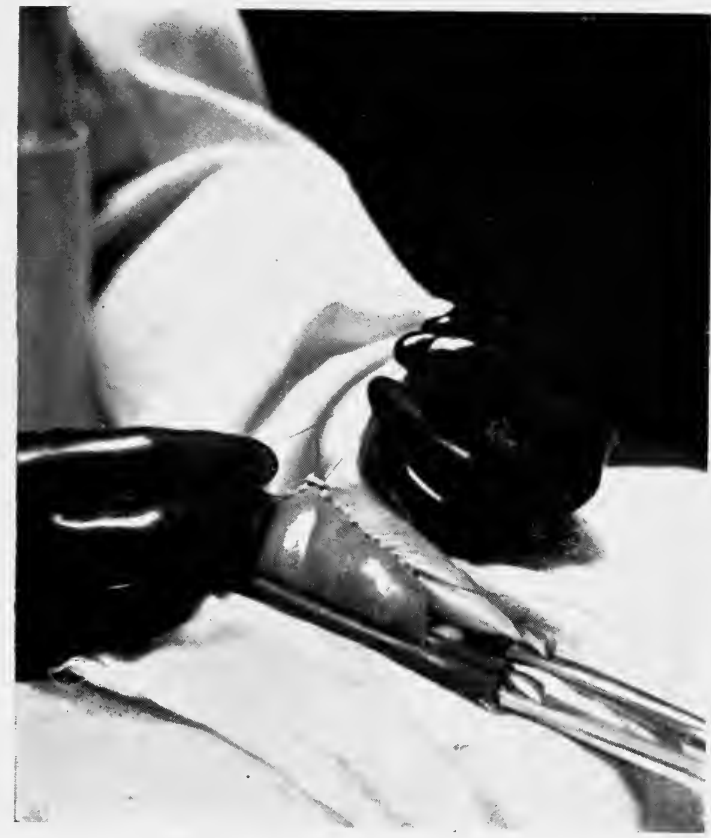

FIG. 11.-The posterior half of the serous suture has been inserted. 

PlATE VI.-ANTERIOR GASTRO-JEJUNOSTOMY.

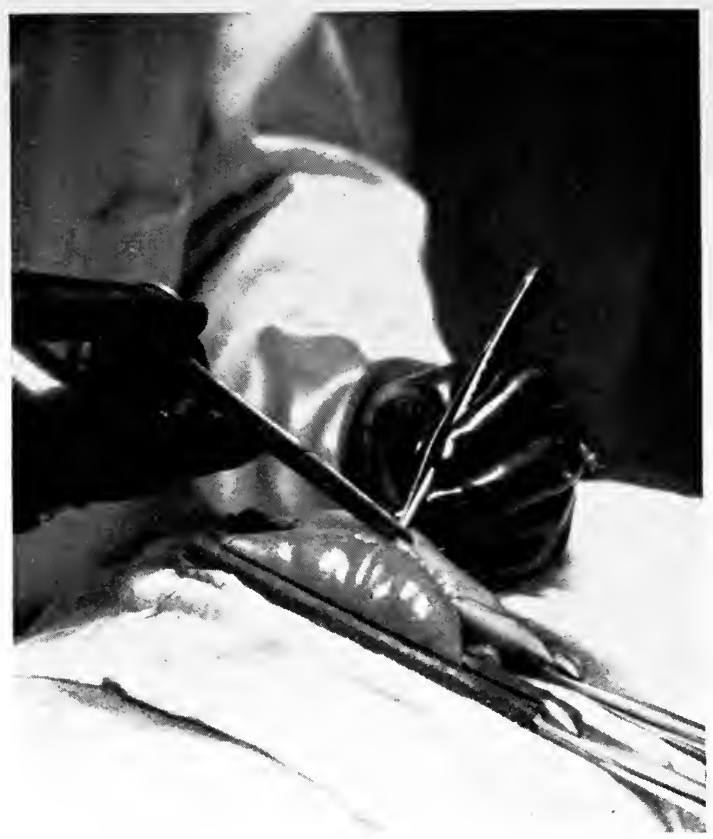

FiG. 12.-Opening the stomach.

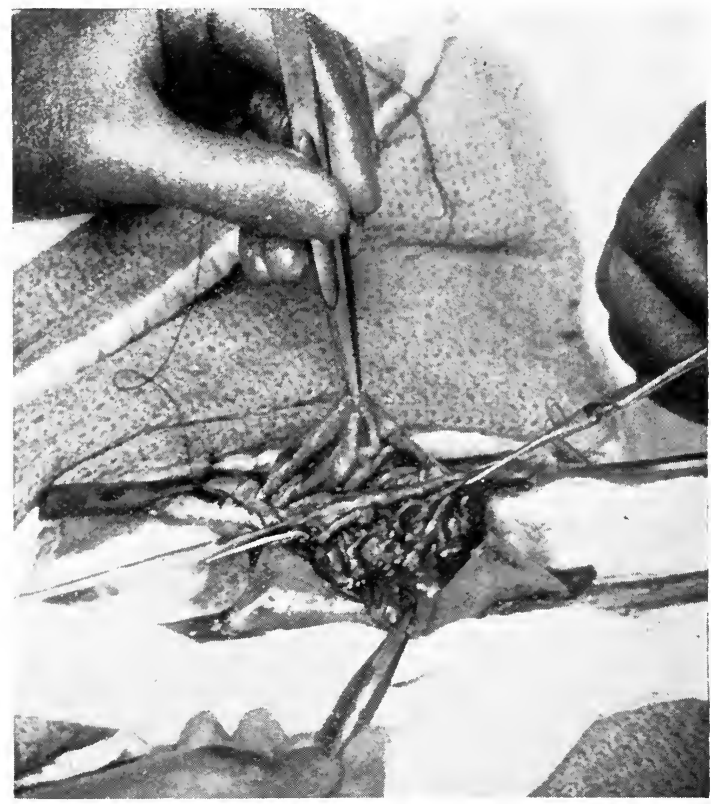

FIG. 13.-Stomach and jejunum opened. The walls of the stomach and jejunum held in apposition by two pairs of Porrier's forceps, ready for insertion of posterior layer of hæmostatic suture. 


$$
\text { - }
$$


Plate VII.-ANTERIOR GASTRO-JEJUNOSTOMY.

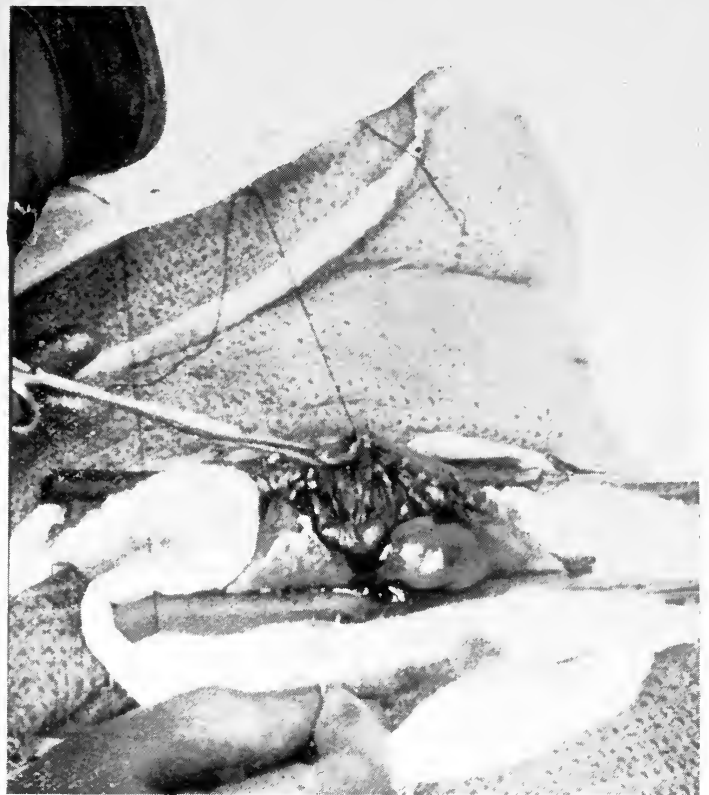

FIG. 14. - The posterior layer of hæmostatic suture nearly completed.

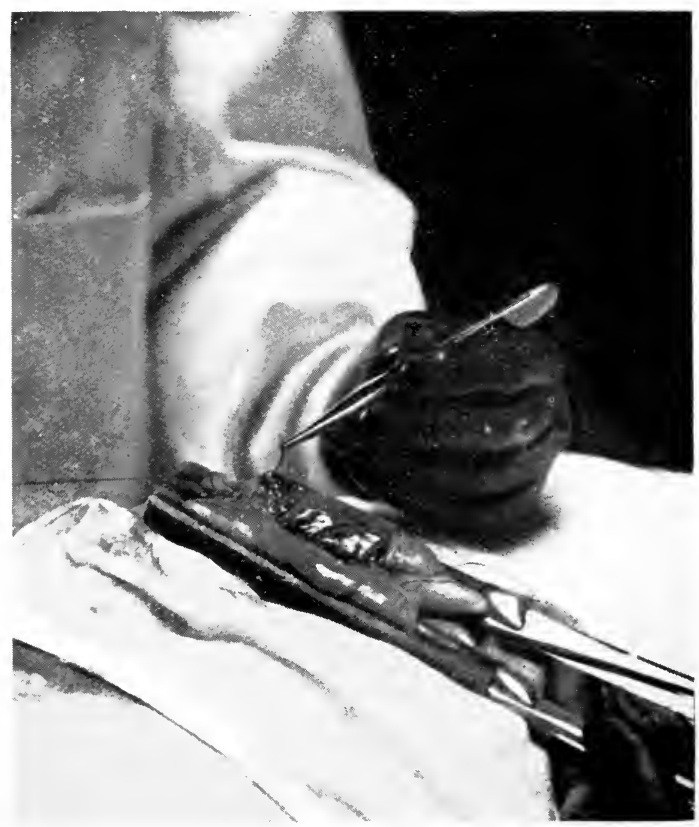

FIG. 15. - The last stitch of the posterior layer. Note the needle emerging from the mucous surface of the stomach. 
Fig. 13). The inner suture is commenced at the same point as the serous suture (Plate VII, Fig. 14), and is continued without interruption to the starting-point.

For the insertion of the posterior layer the needle is passed through the jejunal mucosa, through the wall of the jejunum, then through the peritoneal, muscular, and mucous coats of the stomach. On reaching the right extremity of the opening it is necessary to reverse the direction of the suture. This is accomplished as follows: After passing the stitch at the extreme right end of the opening, the needle emerges on the mucous surface of the stomach (Plate VII, Fig. 15). Then the needle is passed again through the stomach wall from the mucous surface (Plate VIII, Fig. 16), and then the suture is continued by passing the needle through the jejunum from the peritoneal aspect, then through the mucous and muscular coats of the stomach to the peritoneal surface (Plate VIII, Fig. 17). While the anterior layer of the hæmostatic suture is being passed the walls of the stomach and jejunum are held up by means of two pairs of Porrier's forceps, which are applied at the left extremity of the opening and held by the assistant (Plate VIII, Fig. 17).

In this way the mucosa is everted and any bleeding vessels can be seen readily when the clamps are removed.

The careful application of this suture is of great importance for two reasons: first, if the stitches be inserted at too great intervals, or be not pulled sufficiently taut, fatal hæmorrhage may ensue from the mucous membrane; secondly, if the sutures be pulled too tightly, or the mucosa of the jejunum be not in accurate apposition with the gastric mucosa, ulceration will occur along the line of suture (gastrojejunal ulcer).

The stitches should be at intervals of not more than one-eighth of an inch. On reaching the starting-point the 
two ends of the suture are tied, the knot being on the mucous surface, and the ends cut short (Plate IX, Fig. 18).

The clamps, having served their purpose, are now taken off. The mucous membranes of the stomach and jejunum are still visible. If there be any bleeding point on the mucosa it is controlled by passing a through-and-through suture. Next, the outer serous suture is continued round the right extremity of the anastomotic opening, and then in front of the inner suture to the original starting-point. The two ends are then drawn taut and cut short (Plate IX, Fig. 19), the anastomosis gently washed with warm saline solution, and all pads removed. By the completion of the serous suture, the mucous membranes of the stomach and jejunum are buried, and so are visible no longer.

The next step is a most important part of the operation, namely, the application of "anchor" sutures to prevent kinking of the gut, or the formation of a spur. I insert two or three sutures between the stomach and jejunum at each end of the anastomosis (Plate X, Figs. 20 and 21). In this way the abrupt angles which the afferent and efferent limbs of the jejunum make at each end of the anastomosis are changed into a gradual curve, and the patency of the opening between the stomach and efferent loop of the jejunum is assured.

The anastomosed viscera are then returned into the abdomen, and the abdominal wound sewn up as usual.

The operation should be completed in twenty-five or thirty minutes. The points of importance in the operation are:

1. Making a large anastomotic opening, at least three inches in length.

2. Excision of a portion of the gastric mucosa.

3. Careful passing of the hæmostatic suture to secure firm and accurate apposition of the gastric and jejunal mucous membrane.

4. The use of " anchor" sutures. 
Plate VIII.-ANTERIOR GASTRO-JEJUNOSTOMY.

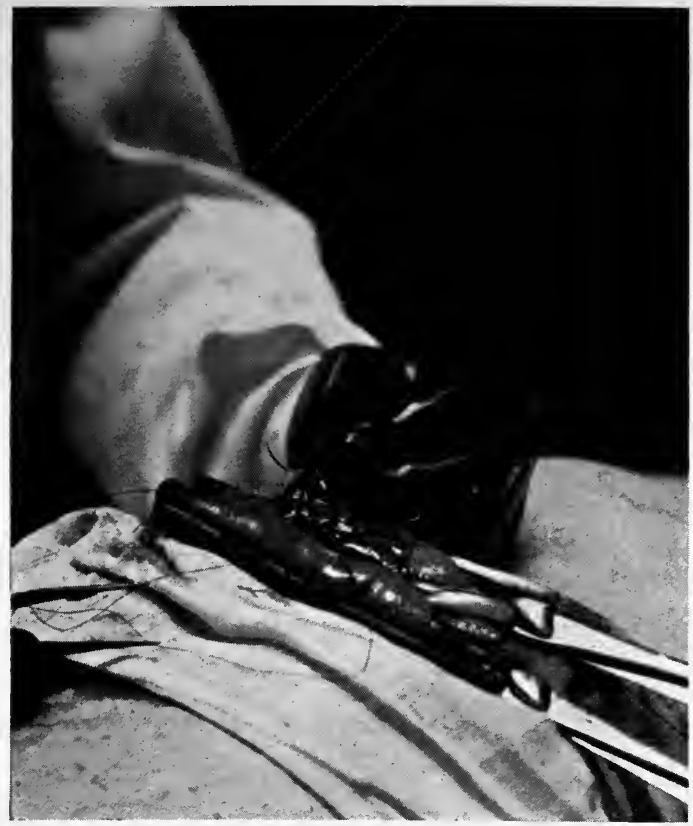

FIG. 16.-Reversal of direction of stitch. The needle is being passed from the mucous surface back again through the stomach wall.

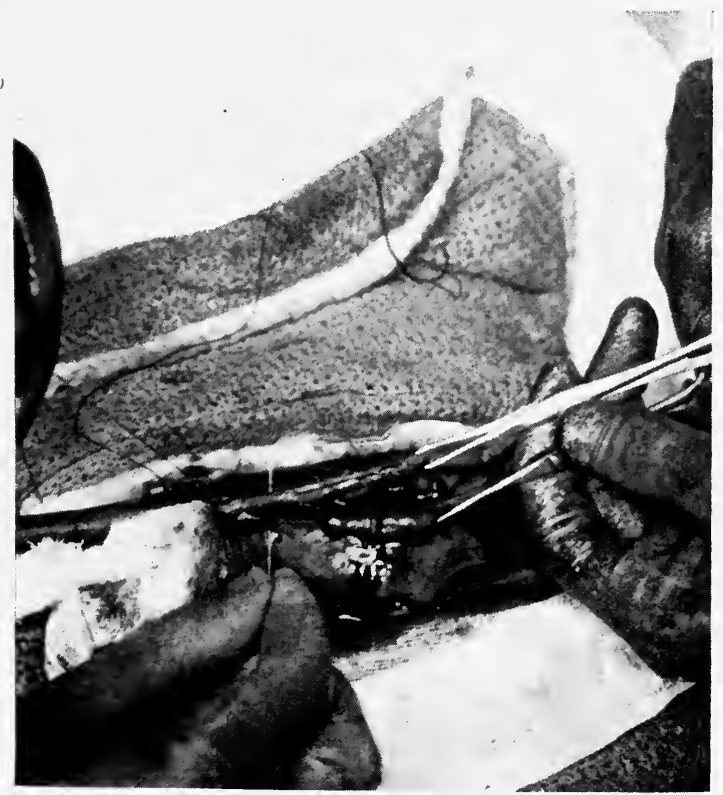

FIG. 17.-The first stitch of the anterior layer. Note that the needle is being passed from the peritoneal surface of jejunum and emerges on peritoneal surface of stomach. 


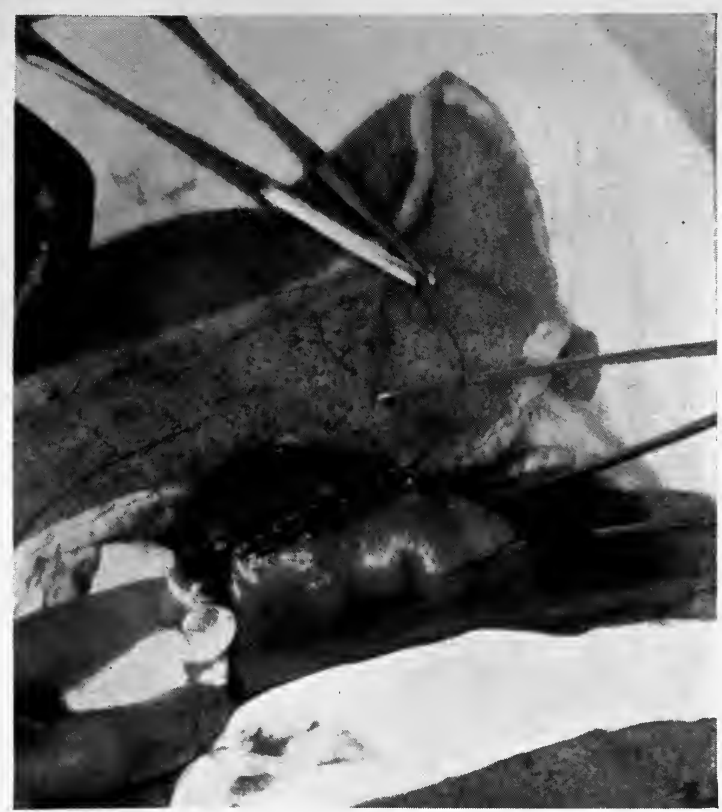

FIG. 18.-The hæmostatic suture is completed. Note that the cut edges of the gastric and jejunal mucous membranes are everted and so are still visible.

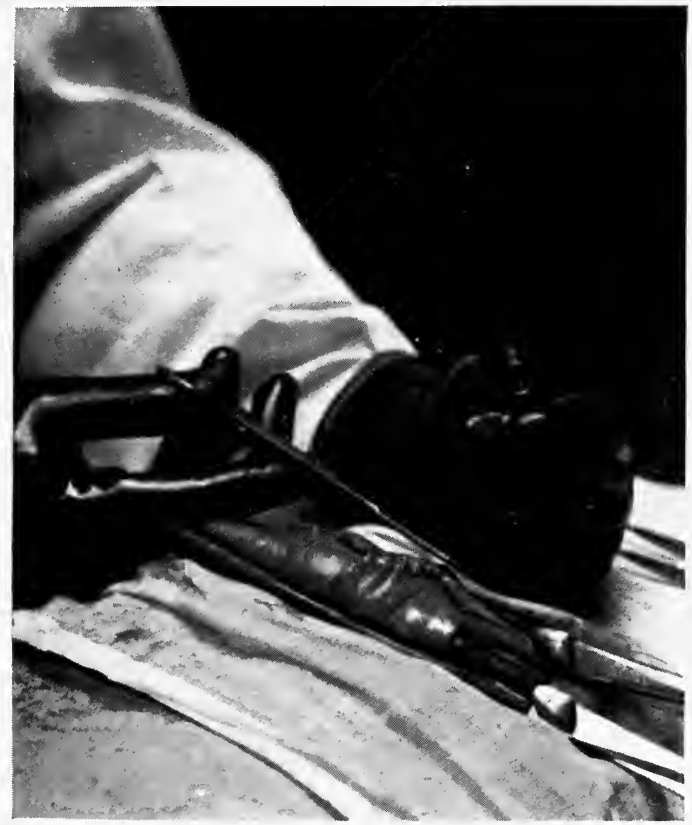

FIG. 19.-The serous suture completed. The edges of the gastric and jejunal mucous membranes are now invisible. 


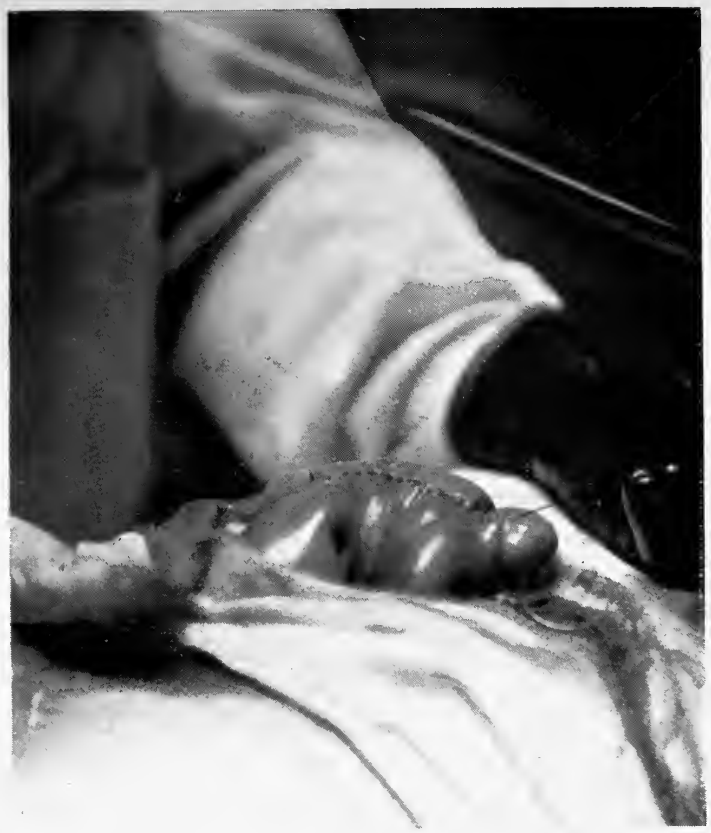

FIG. 20.-The insertion of two " anchor sutures," at left extremity of anastomosis.

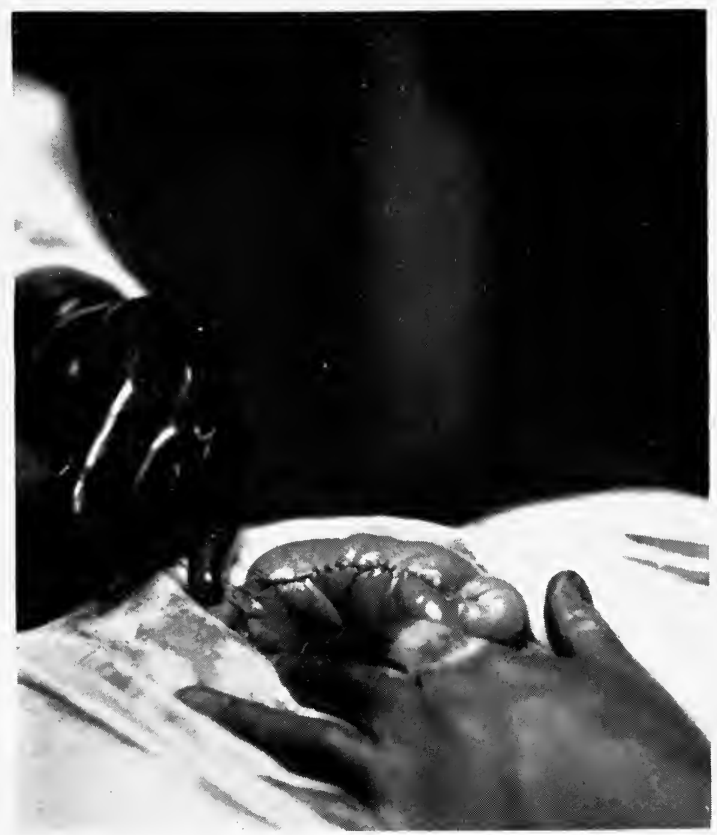

FIG. 21.-The insertion of two "anchor sutures" at the right extremity of anastomosis. 


\section{OPERATIONS ON THE STOMACH}

Posterior Gastro-jejunostomy.-The method of gastrojejunostomy in general use is spoken of as the posterior "no-loop" operation. This description is not strictly accurate. It is impracticable to make the anastomosis absolutely close to the duodeno-jejunal junction. In practice

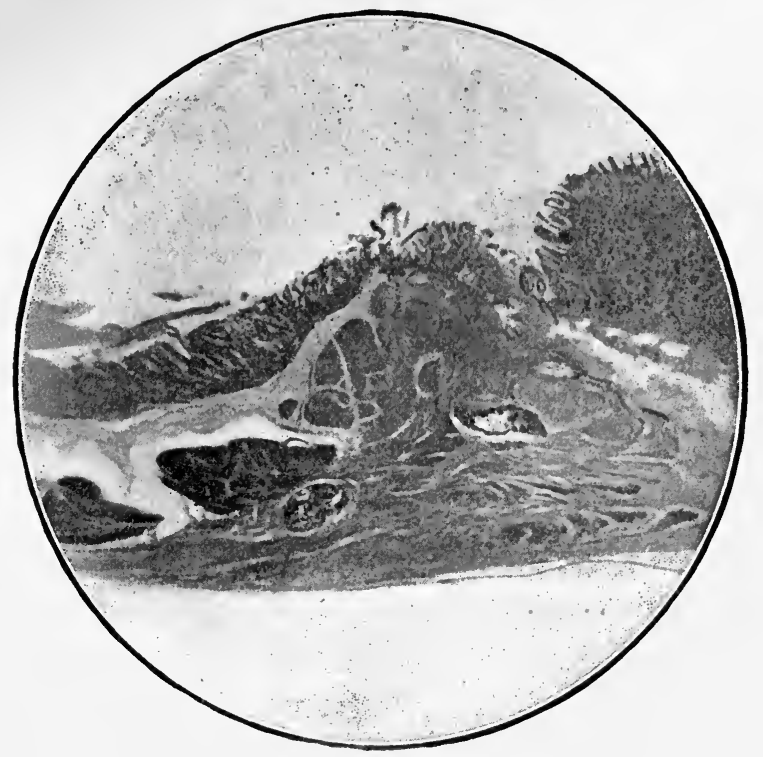

FIG. 22.-Section of the junction between the stomach and jejunum five months after gastro-jejunostorny.

The continuity of the mucous membrane is well shown.

about an inch of jejunum intervenes between the origin of the jejunum and the point at which the junction is made. It is more correct, therefore, to speak of the posterior " shortloop" operation. It is performed in the following manner :

After opening the peritoneal cavity, the stomach and transverse colon are drawn gently upwards, and two fingers introduced into the abdomen seek the origin of the jejunum close to the left of the spine at the root of the transverse meso-colon. 
The posterior surface of the stomach is exposed in the following manner: A bloodless portion of the meso-colon, close to the root of the jejunum, is seized with a pair of forceps. The forceps are lifted up and a nick made by the side of them with a pair of scissors. The opening is enlarged by stretching until it is about four inches in length.

In Fig. 23, Plate XI, the posterior wall of the stomach is exposed, the edges of the meso-colon are held in forceps, and the origin of the jejunum is grasped by the thumb and forefinger of the right hand.

A portion of jejunum four inches long, close to its origin, is clamped with a pair of long forceps, the blades of which are sheathed with rubber (Plate XI, Fig. 24). The jejunum is clamped so that its direction is from right to left-that is, so that the portion nearest its origin is at the right end of the clamp.

A portion of the posterior wall of the stomach is drawn up through the hole which has been made in the transverse meso-colon, and is clamped with a second pair of long forceps. The portion clamped should be at least four inches long, and should be as near the pyloric end of the stomach as is possible. The clamp is applied to the stomach obliquely from above downwards and to the right. [Thus when the anastomosis has been completed and the stomach returned to the abdomen, the direction of the anastomotic opening will be oblique, extending from above downwards and to the left. The anastomosis is therefore anti-peristaltic.]

The transverse colon is now returned into the abdomen, the handles of the forceps are taken charge of by the assistant, and the viscera to be anastomosed are surrounded with pads. Nothing is visible except the portions of stomach and jejunum to be anastomosed (Plate XII, Fig. 25). A guide suture is inserted at each end of the clamped viscera (Plate XII, Fig. 26). These sutures are at least four inches. 
Plate XI.-POSTERIOR GASTRO-JEJUNOSTOMY.

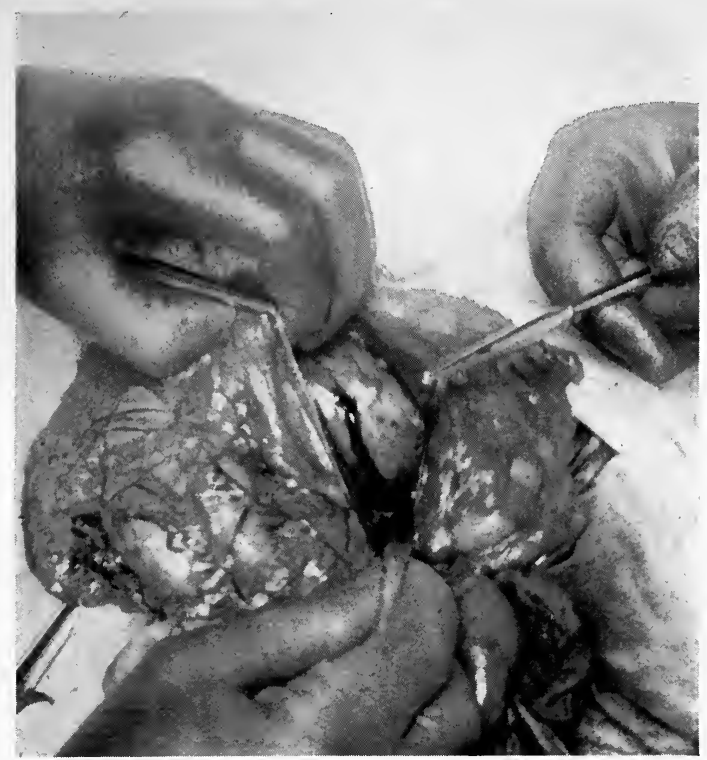

FIG. 23.-Posterior surface of stomach exposed; the origin of jejunum is shown grasped by the thumb and forefinger of right hand.

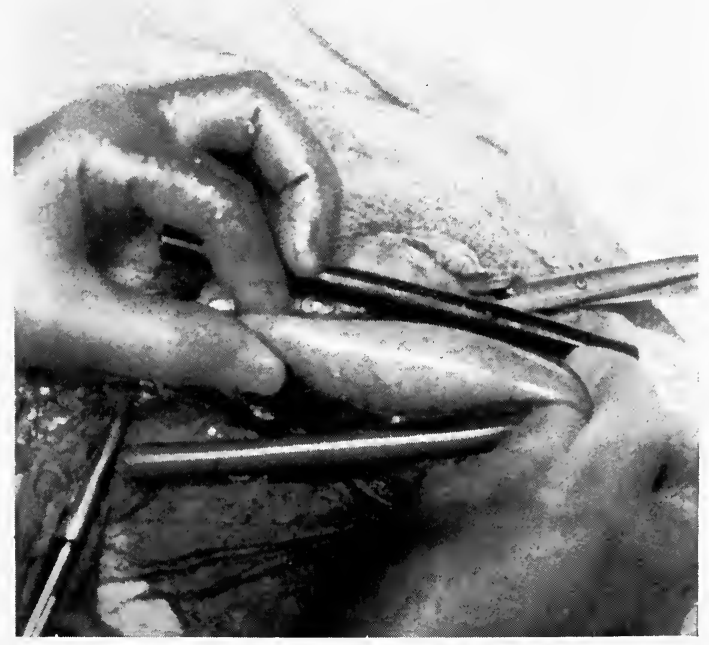

FIG. 24. - The jejunum is held while the clamps are applied by the assistant. 
Plate XiI.-POSTERIOR Gastro-JeJUNOSTOMY.

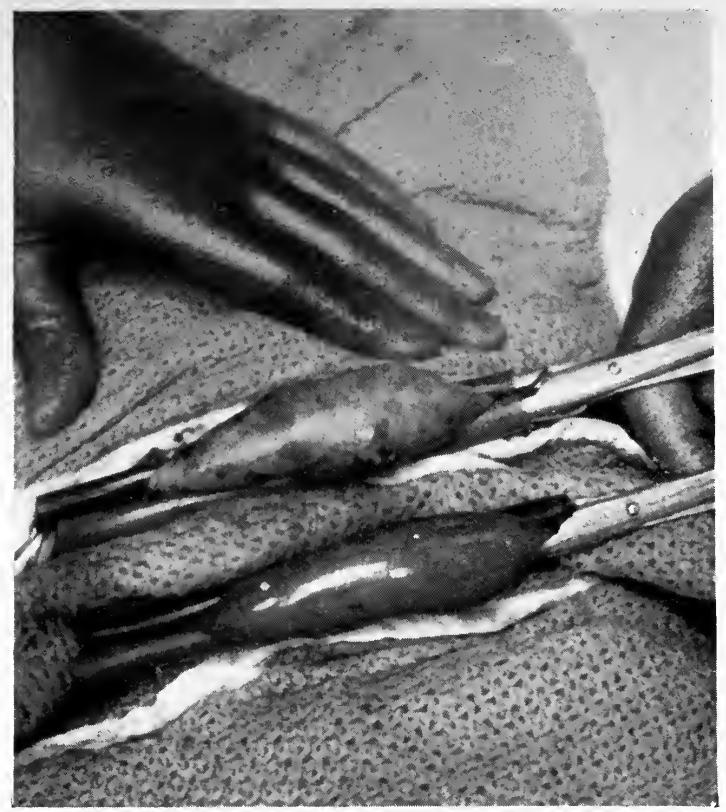

FIG. 25.-Portions of stomach and jejunum clamped and surrounded with pads.

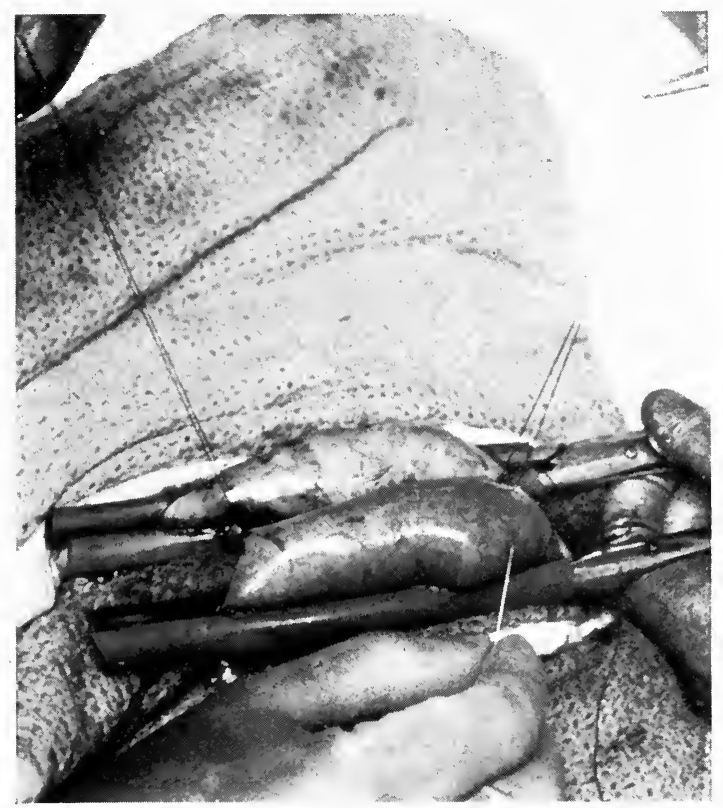

FIG. 26. - The clamped portions of viscera united at their extremities by the insertion of two guide sutures. 
Plate XIII.-POSTERIOR GASTRO-JEJUNOSTOMY.

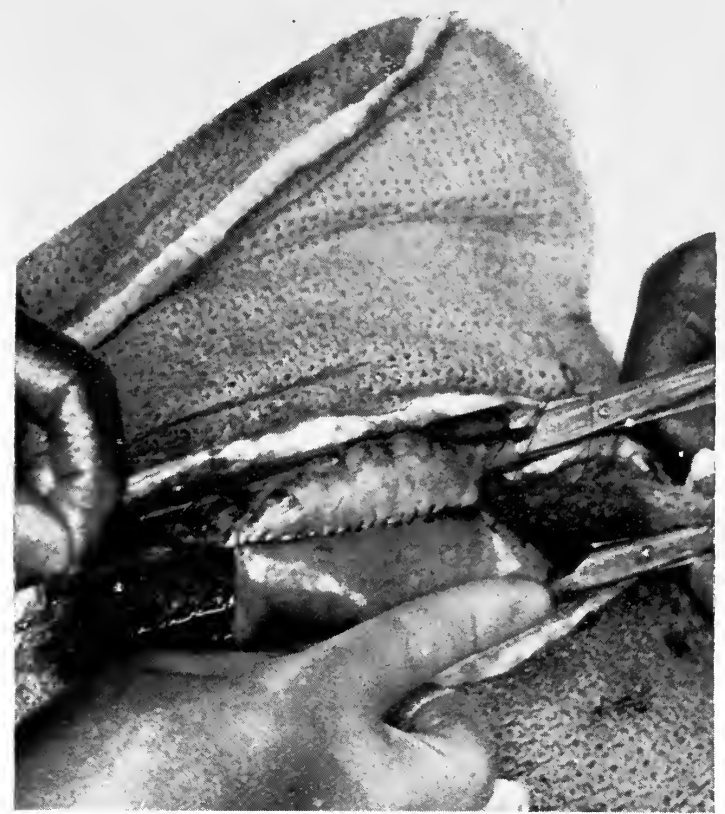

FIG. 27.-The posterior half of the serous suture complete.

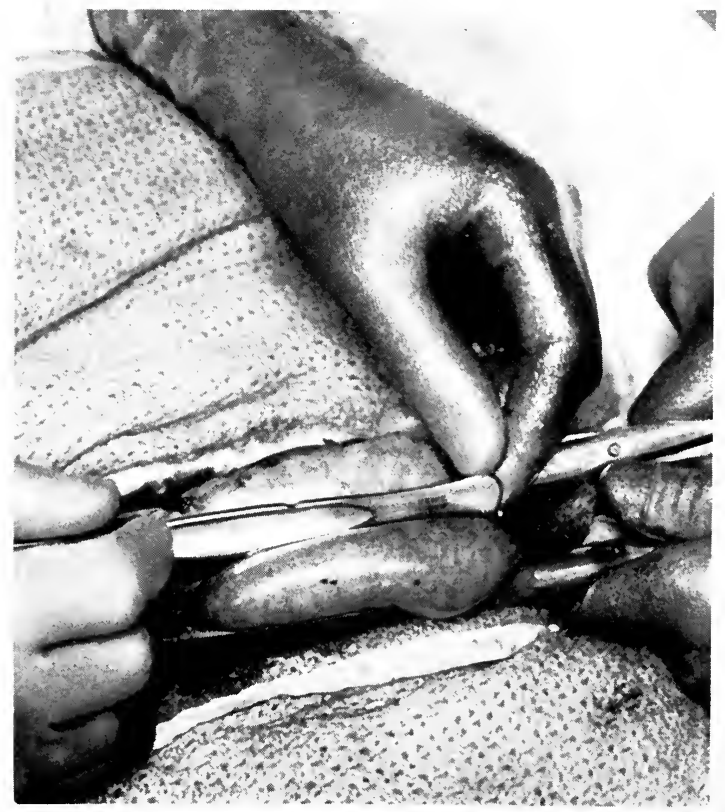

FIG. 28.-Measuring, to make sure that the portion sutured measures four inches. 
Plate XIV.-POSTERIOR GASTRO-JEJUNOSTOMY.

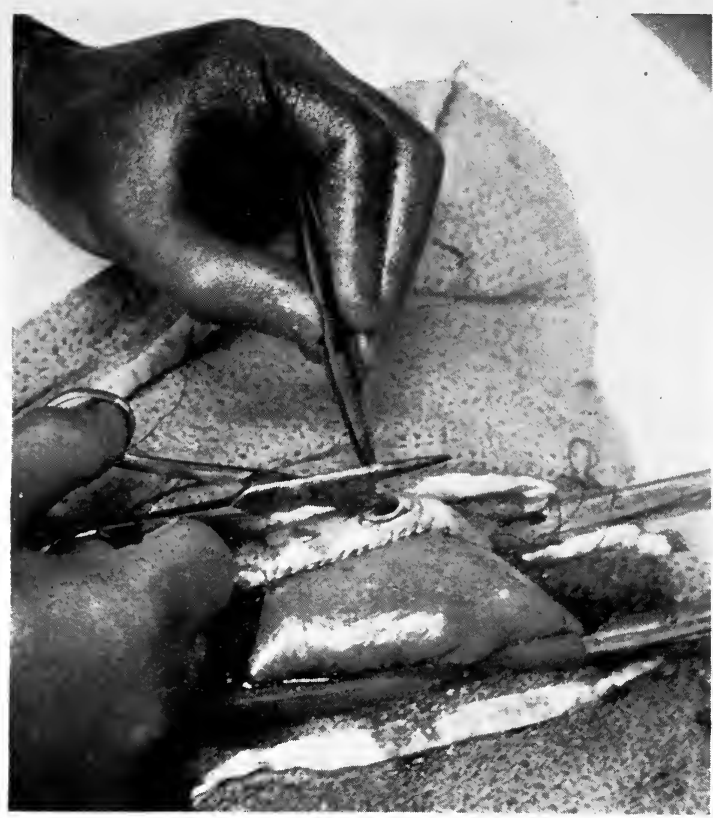

FIG. 29.-Opening the stomach.

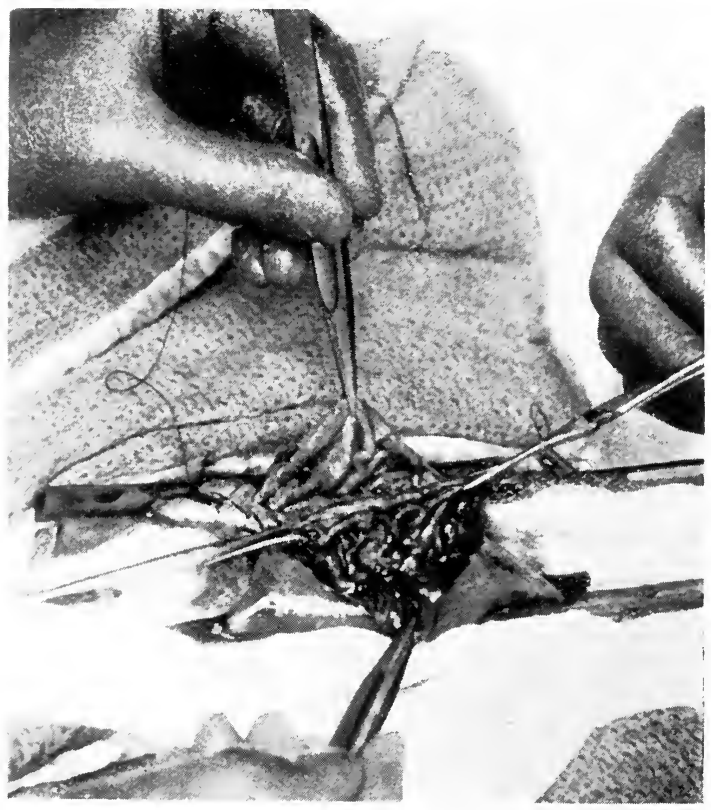

Fig. 30.-Stomach and jejunum opened. The walls of stomach and jejunum held in apposition by two pairs of Porrier's forceps ready for insertion of posterior layer of hæmostatic suture. 
apart, and their object is to ensure that the line of suturing shall be straight.

The anastomosis is now effected by means of two continuous sutures, an inner hæmostatic suture penetrating all the coats of the viscera, and an outer approximating suture including the peritoneal, muscular, and submucous coats. For both sutures I use a very fine black Irish linen thread.

The outer serous suture is commenced at the left end of the clamped portions of the stomach and jejunum, and is continued towards the right for four inches (Plate XIII, Fig. 27).

I have a notch cut on the handles of my scalpels $2 \frac{1}{2}$ inches from the end, so that by using this as a measure (Plate XIII, Fig. 28), I can be certain that the line of suture is of adequate length. Without being unthreaded, the needle is clamped with a pair of pressure forceps and laid upon the muslin sheet above the wound, ready for use later. I find my "grip-eyed" needles of great advantage, as they cannot become unthreaded accidentally.

A portion of the stomach wall, as close to the suture line as possible, is picked up with a pair of dissecting forceps, and the stomach is opened by cutting into it with a small pair of scissors (Plate XIV, Fig. 29). The incision is then prolonged in both directions until the opening measures three inches. A similar opening is made in the jejunum. I attach great importance to making the anastomotic opening of sufficient size.

As the viscera are opened the mucous membrane pouts into the wound. With a pair of scissors, about a quarter of an inch of the mucous membrane is removed on each side of the opening into the stomach. As a rule I do not remove any of the jejunal mucosa.

The inner hæmostatic suture, penetrating all coats of the stomach and jejunum, is now introduced. The posterior cut edges of the walls of the stomach and jejunum are kept in 
apposition by means of two pairs of Porrier's forceps, one pair being applied at each end of the anastomotic opening (Plate XIV, Fig. 30).

The inner suture is commenced at the same point as the serous suture, and is continued (Plate XV, Fig. 31) without interruption to the starting-point.

In the insertion of the posterior layer the needle is passed through the mucosa of the jejunum, then through the wall of the jejunum, the wall of the stomach, and finally through the mucosa of the stomach; but on reaching the right extremity of the opening it is necessary to reverse the direction of the suture. This is accomplished as follows: After passing the stitch at the extreme right of the opening the needle emerges on the mucous surface of the stomach (Plate XV, Fig. 32). Then the needle is passed back again through the stomach wall from the mucous surface (Plate XVI, Fig. 33), and then the suture is continued by passing the needle through the jejunum from the peritoneal aspect, then through the mucous and muscular coats of the stomach to the peritoneal surface (Plate XVI, Fig. 34). The passing of the suture is facilitated by holding up the left extremities of the incisions into the stomach and jejunum by means of two pairs of Porrier's forceps (Plate XVI, Fig. 34). By passing the suture in this manner the edges of the mucosa are everted, and any bleeding vessels can be seen readily when the clamps are removed. The careful application of this suture is of great importance because, first, if the stitches be not inserted sufficiently closely together, or be not pulled sufficiently taut, fatal hæmorrhage may ensue from the mucous membrane; secondly, if the sutures be pulled too tightly, or the mucosa of the jejunum be not in accurate apposition with the gastric mucous membrane, ulceration will occur along the line of suture (gastro-jejunal ulcer). The stitches should be at intervals 
Plate XV.-POSTERIOR GASTRO-JEJUNOSTOMY.

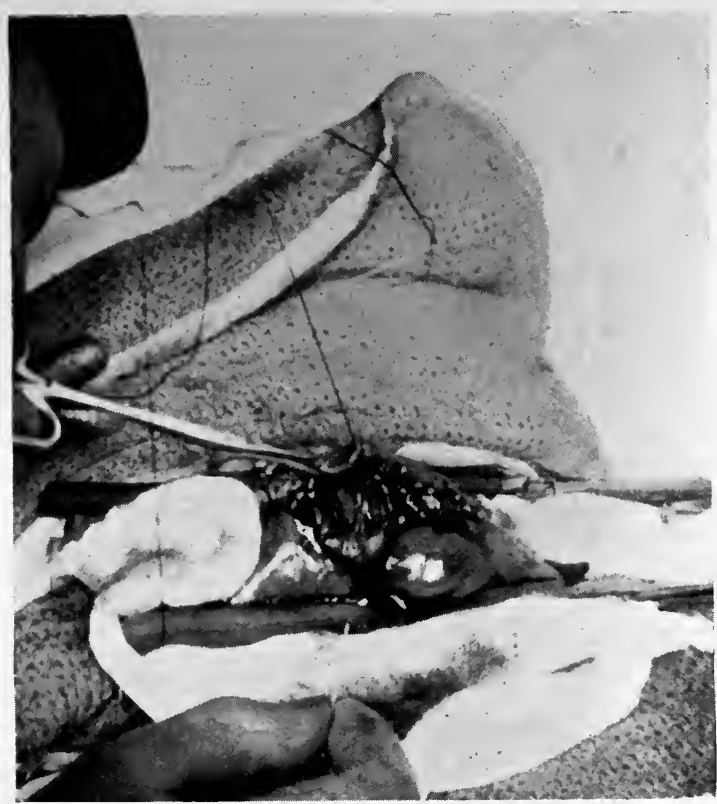

FIG. 31.-The posterior layer of the hæmostatic suture nearly completed.

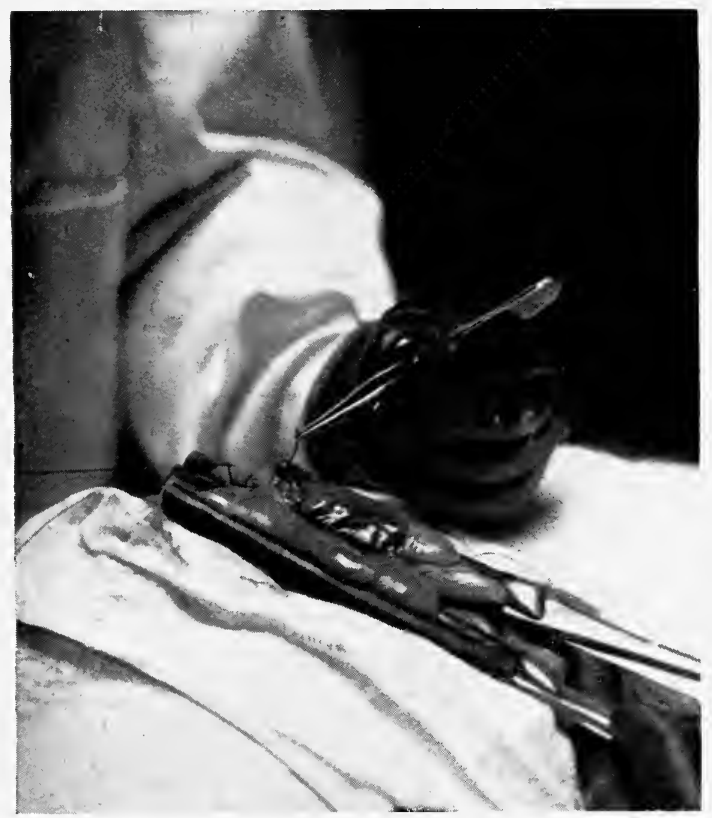

FIG. 32.-The last stitch of posterior layer. Note the needle emerging on mucous surface of the stomach. 
PI.ATE XVI.-POSTERIOR GASTRO-JEJUNOSTOMY.

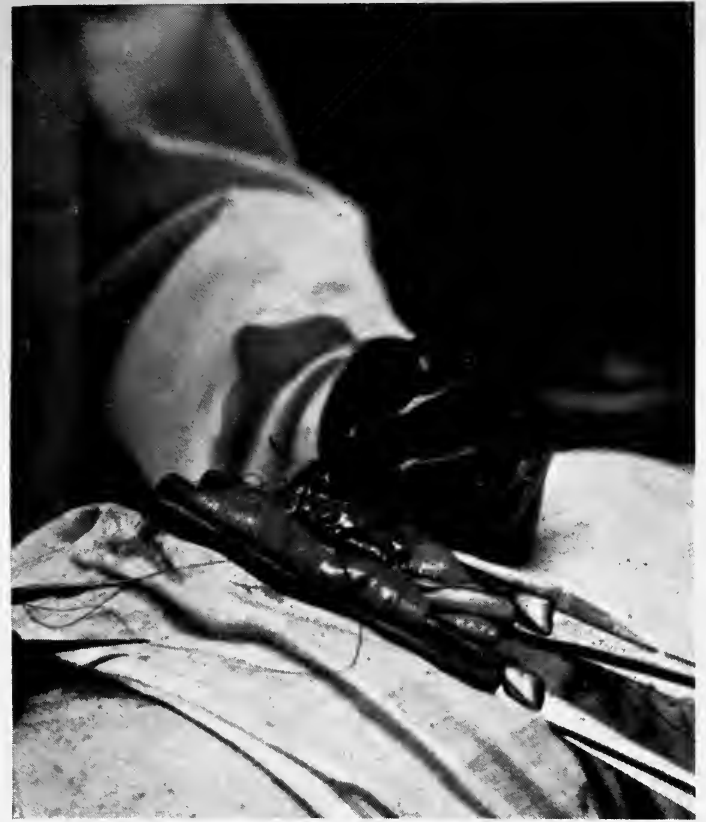

FIG. 33.-Reversal of direction of stitch. The needle is being passed back again through the stomach wall from the mucous surface.

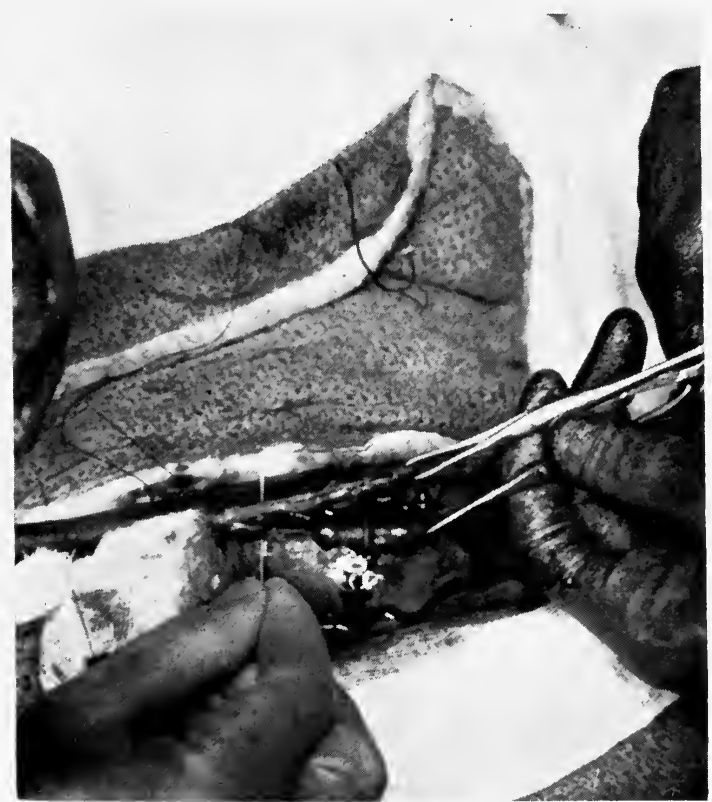

FrG. 34.-The first stitch of anterior layer. The needle is being passed from peritoneal surface of jejunum and emerges on peritoneal surface of stomach. 


\section{-}

.

+

. 


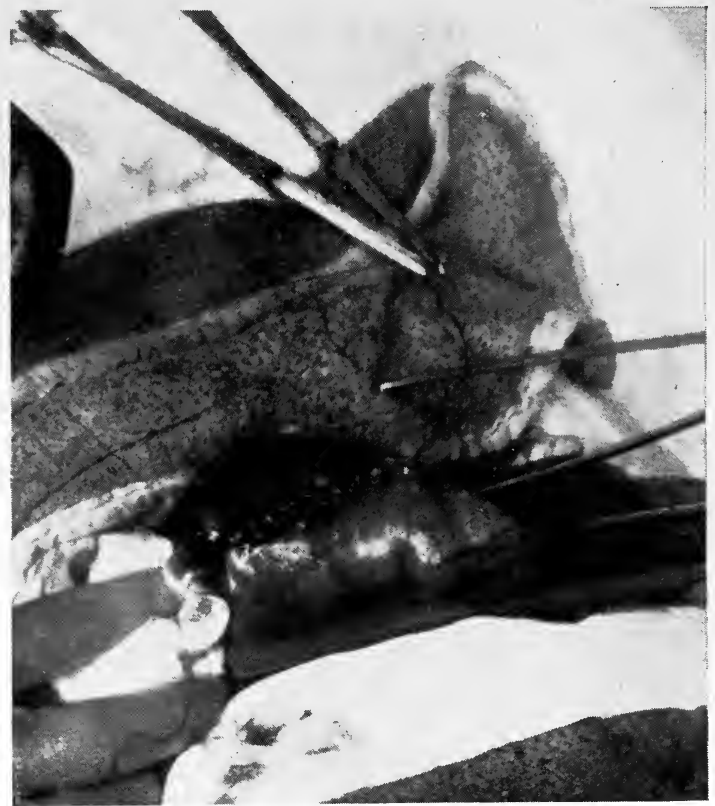

FIG. 35. - The hæmostatic suture completed. Note that the edges of the gastric and jejunal mucosa are everted and so are still visible.

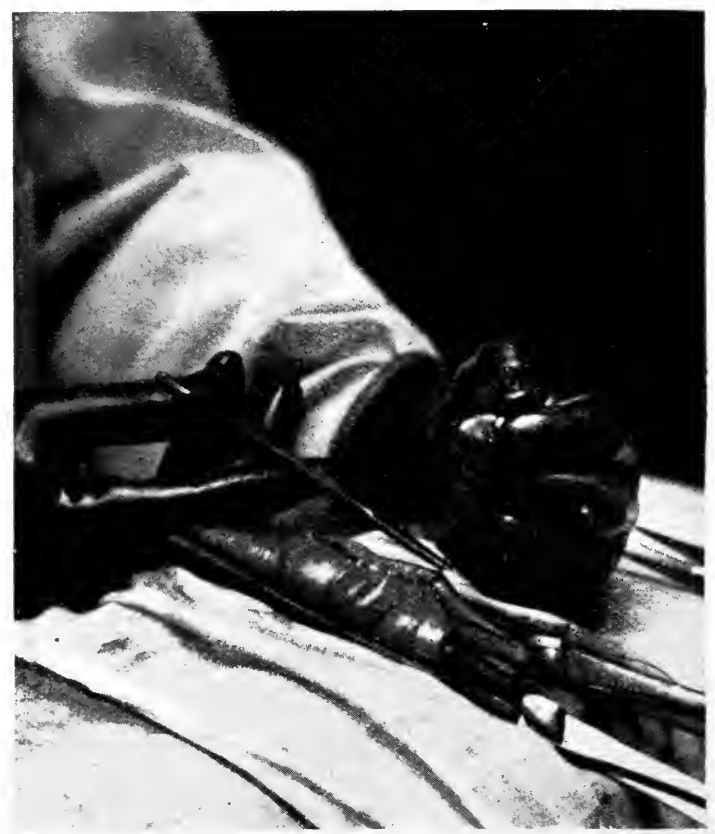

FIG. 36. - The anastomosis completed. The mucous membranes have been buried by the completion of the serous suture. 
of not more than one-eighth of an inch. On reaching the starting-point the two ends of the suture are tied, the knot being on the mucous surface, and the ends cut short (Plate XVII, Fig. 35). The clamps, having served their purpose, are now taken off.

If there be any bleeding point on the mucosa, it is controlled by passing a through-and-through suture.

The outer serous suture is now resumed, and is continued round the right extremity of the anastomotic opening and then in front of the inner suture to the original startingpoint.

The two ends are drawn taut and cut short (Plate XVII, Fig. 36), the anastomosis gently washed with warm saline solution, and all pads are removed.

The completion of the serous suture buries the everted mucous membranes, which are visible no longer.

The edges of the hole in the meso-colon are now fixed to the stomach round the anastomosis by means of four sutures, so that no gap is left to allow prolapse of the intestine through the hole in the meso-colon and so lead to an internal hernia.

The abdominal wound is sewn up in the usual way.

Roux's Gastro-jejunostomy " En-Y."-In this method, first suggested by Wölfler and improved and popularised by Professor Roux, the jejunum is cut across nine or ten inches from its origin, and the distal end anastomosed to the posterior surface of the stomach through an aperture made for the purpose in the transverse meso-colon. Then the proximal end of the jejunum is implanted into the side of the distal portion three inches below its attachment to the stomach.

The anastomosis to the stomach may be made equally well on the anterior surface of the viscus.

The advantage claimed for Roux's operation is that it prevents regurgitant vomiting. This is not absolutely true, 
as $I$ have known at least two cases in which regurgitant vomiting was present after this method had been employed.

It is a complicated and difficult operation, and takes at least from fifteen to twenty minutes longer to perform than a simple gastro-jejunostomy. The chief, and to my mind fatal objection to it is, that the danger of jejunal ulcer is increased, as a portion of jejunum is exposed to the action

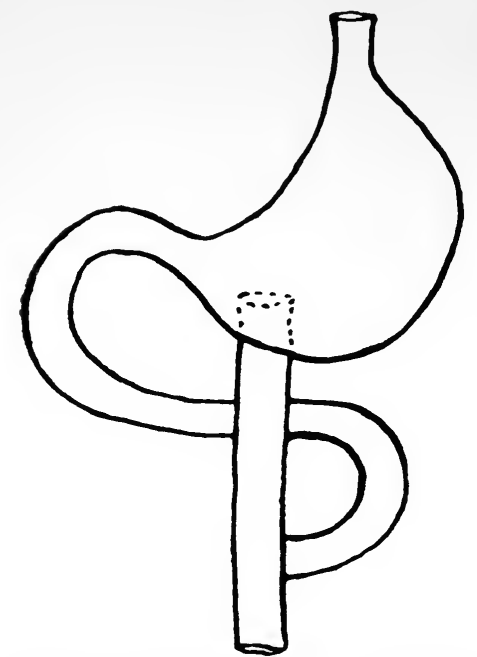

FIG. 37.-Gastro-jejunostomy " En-Y" (Roux's method)

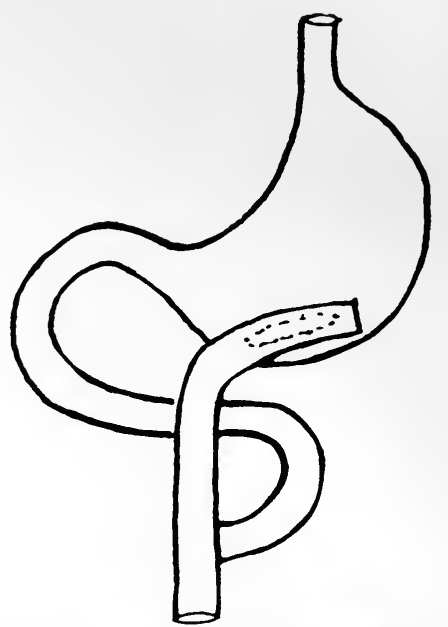

FIG. 38.-Gastro-jejunostomy " En-Y" (Moynihan's modification)

of the gastric juice, without the neutralising effects of the bile and pancreatic juice.

The method is not one to be recommended as the operation of choice, but in the treatment of jejunal ulcer, and regurgitant vomiting, it is sometimes the only procedure available. I have performed it for these conditions on several occasions. The results are satisfactory on the whole, but it has seemed to me that the opening from the stomach is not sufficiently large, and so a certain degree of stasis is present after the operation.

This objection can be overcome by anastomosing the 
jejunum to the stomach laterally, as suggested by Sir Berkeley Moynihan, instead of in the manner originally carried out by Professor Roux.

I consider Sir Berkeley's modification to be preferable to the older method. The operation is performed as follows : A portion of the stomach is clamped as for the ordinary anterior operation. Two clamps are then placed on the jejunum eight or nine inches from its origin, and the intestine divided between the clamps, the cut being extended into the mesentery. The distal end of the jejunum is now closed by two continuous sutures, an inner, penetrating all coats, and an outer serous suture.

This portion of the jejunum is anastomosed to the stomach exactly as in an anterior gastro-jejunostomy. Finally, the proximal end of the jejunum is implanted into the left side of the distal portion of the jejunum, three inches below its attachment to the stomach.

\section{GASTRO-PLASTY AND GASTRO-GASTROSTOMY}

Gastro-plasty is the application to the stomach of the principle of the operation of pyloroplasty. Its rôle is a very limited one. It has been performed frequently for hourglass stomach, but the after-results are not satisfactory. Relapse ensues in 25 per cent. of the cases. To guard against relapse after gastro-plasty, gastro-jejunostomy should be performed in addition. If, however, a gastro-jejunostomy be performed, the gastro-plasty, in most cases, is unnecessary.

Gastro-plasty is performed as follows: After opening the abdomen the stomach is packed round with pads to prevent contamination of the peritoneum. An incision $3 \frac{1}{2}$ to 4 inches is made in the long axis of the stomach, having its centre at the constriction. The edges of the stomach at the middle of the incision are seized with forceps. These are gently 
pulled apart, so as to change the direction of the incision from the long axis to the vertical axis of the stomach. The forceps are held by an assistant, and the incision is sewn up with two continuous sutures of linen thread, an inner suture penetrating all coats, and an outer serous suture. The abdominal wound is closed in the usual manner without drainage.

Gastro-gastrostomy. - In gastro-gastrostomy the pouches on each side of the constriction are anastomosed, so as to make a free passage from the cardiac to the pyloric pouch below the contracted isthmus.

The operation is performed in the following manner: The stomach is clamped on either side of the constriction. A continuous serous suture is then passed from the constriction to the greater curvature, so as to bring into apposition the cardiac and pyloric pouches below the constricted area. The needle is then laid aside, and incisions are made into each pouch in front of the suture line, so as to open the interior of both pouches. All redundant mucosa is excised, and a continuous suture of linen, penetrating all coats, is passed right round the opening. Then the original serous suture is continued in front of the anastomosis. The technique is exactly the same as that of gastro-jejunostomy except that stomach is united to stomach, instead of jejunum to stomach.

Gastro-gastrostomy is open to the same objections as gastro-plasty, namely the frequency with which relapse ensues. In at least 30 per cent. of the cases the patient does not obtain permanent relief.

\section{GASTRECTOMY}

Gastrectomy may be partial or complete.

(a) Partial Gastrectomy.-In partial gastrectomy for cancer, at least one inch of the duodenum, one-half of the 
greater curvature, and the whole of the lesser curvature should be removed, together with the glands lying along the lesser curvature, and those connected with the pyloric half of the greater curvature.

Personally, I prefer to make the section of the greater curvature at the junction of the middle third with the cardiac third, so that two-thirds of the stomach are removed.

The steps of the operation are as follows :

(i) An opening is made through the gastro-hepatic

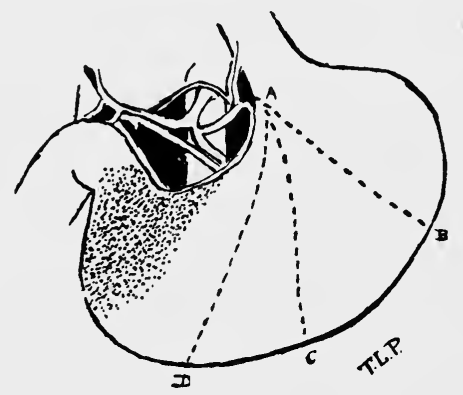

Fra. 39.-Lines of incision used in partial gastrectomy.

$A B$-Line of incision advisable except in very early cases (Mayo, Moynihan, the author).

$A C$-Line of incision in very early cases (Mikulicz).

$A D$-Line of incision of Hartmann.

omentum so as to open the lesser peritoneal sac. The limits of the growth are explored thoroughly, so as to ascertain whether the case is suitable for excision. Adhesion to the pancreas is not necessarily a contra-indication to resection if the conditions be favourable otherwise. According to Haberkant, removal of a portion of the pancreas raises the mortality rate to 76 per cent., and Mikulicz gives 74 per cent. as the death-rate in such cases. Dr. W. J. Mayo does not consider that there is any special mortality due to operable pancreatic complication.

In one of my cases, there was a freely movable, large mass attached to the lesser curvature and pylorus, which I took to be infiltrated omentum. When proceeding to cut 
away the stomach after the ligation of the vessels and the dissection had been completed, I found to my dismay that the mass consisted of the head of the pancreas, which had been lifted out of its bed by the pyloric growth. There was no alternative but to remove about two-thirds of the head of the pancreas with the stomach. This I did, and the patient made an excellent recovery, complicated only by a pancreatic fistula which persisted for three weeks. The operation was five years ago, and the patient has been in excellent health ever since.

Of greater importance is the extent of glandular infection. Enlargement of glands does not necessarily mean that they are infected with carcinoma. This cannot be determined except by microscopical examination. If, therefore, there be enlarged glands which cannot be removed completely, one of them should be excised and submitted to microscopical examination, the resection postponed until a later date, and the gastro-jejunostomy performed meanwhile.

(ii) Having decided that the case is one suitable for resection, the next step is to make the anastomosis between the jejunum and the stomach. Most operators leave this step until the last. In my Hunterian lectures I suggested that the gastro-jejunostomy should be performed before the resection. My reasons for this are as follows: First, if the gastro-jejunostomy be left to the last there is a risk that the surgeon may be tempted to perform it hurriedly, owing to the condition of the patient; secondly, after the gastrojejunostomy has been completed, the surgeon is in a better position to judge how the patient will stand the additional operation of resection; thirdly, the gastro-jejunostomy is performed more easily before resection; and fourthly, in the case of a tumour of doubtful nature, or when enlarged, inoperable glands are present, the time occupied can be utilised to freeze, and examine microscopically, a portion of 
the tumour, or of the suspicious gland. Such investigation, however, requires a pathologist specially skilled in such work, and the report is of most value if it negative malignant disease. I do not act on a positive report of cancer made under these conditions, unless I feel sure from the clinical aspect of the case that the growth is malignant.

The only objection to performing the gastro-jejunostomy first is, that the site chosen for the anastomosis may be in the way of an adequate resection. This objection is met by making the anastomosis as far to the left as is possible.

Whether an anterior or posterior gastro-jejunostomy be performed is immaterial. If the resection is to be a wide one, usually it is advisable to make the gastro-jejunostomy on the anterior wall of the stomach. Whichever method be selected, it is performed in exactly the same way as described previously.

The surgeon now decides whether he will complete the operation by performing resection, or whether he will postpone the remainder of the operation until a later date. There is much to be said for the operation à deux temps, as suggested originally by Tupolske. The chief argument in favour of it is, that by delaying the resection the patient can be fed up, and so be in a better condition to undergo the severer operation. I think, however, this depends on the type of the growth. Those patients who have a hard growth at the pylorus, which has caused partial obstruction, and so resulted in great emaciation, undoubtedly will be benefited by the gastro-jejunostomy, and will put on weight rapidly. Those patients, on the other hand, who have a soft ulcerating or fungating growth, and are suffering from toxæmia rather than from obstruction, will not improve to any extent, and so delay will not improve necessarily their prospects of recovery.

Then again, if the resection be postponed, recent adhesions, 
due to the first operation, are often troublesome, and may make what would have been, in the first instance, an easy resection, a very difficult one. Another objection to postponing the resection is, that sometimes the patient improves so rapidly that the second operation is declined. To a sensible patient, however, the position can be explained beforehand. If, at the completion of the gastro-jejunostomy, the patient's condition be fairly satisfactory, I think it is better to complete the operation.

(iii) If the surgeon decide to perform partial gastrectomy, the next step is the ligation of the four blood-vessels, the gastric, the superior pyloric, the gastro-duodenal, and the left gastro-epiploic arteries.

The stomach is drawn well downwards and to the right, and the gastric artery is felt for with the left forefinger. A curved needle armed with a double thread of No. 2 catgut is now passed between the artery and the stomach as high up as possible. The artery is tied in two places, and divided below the ligature with a pair of scissors, the omentum being divided at the same time right up to the stomach.

(iv) The pyloric artery is sought for and tied with a double ligature, at a point as far away as possible from the duodenum, and divided between the two ligatures. From the point of ligation the gastro-hepatic omentum is divided with scissors, as close to the liver as possible, until the point of ligation of the gastric artery is reached.

(v) From the point of ligation of the gastric artery the glands and fat are dissected downwards along the lesser curvature for a distance of $1 \frac{1}{2}$ inches. This is an important step in the operation, as to prevent recurrence it is essential to remove the whole of the lesser curvature and attached glands. Similarly, the fat and glands lying above the duodenum are dissected off the duodenum, from the right as far as the pylorus. By these dissections, the duodenum is 
freed above, and a clear space is left at the left extremity of the lesser curvature.

(vi) Next, the gastro-colic omentum is dealt with. The left gastro-epiploic vessels are ligatured to the left of the proposed line of section. To the right of this point the gastro-colic omentum is dissected off the greater curvature for a distance of $1 \frac{1}{2}$ inches. The gastro-colic omentum is next ligatured in sections as close to the transverse colon as possible, so that all glands are left attached to the stomach. If the glands lying along the transverse colon be infected with cancer, the transverse colon ought to be resected, otherwise recurrence almost certainly will ensue (see p. 237).

In ligaturing the gastro-colic omentum great care must be taken not to wound the middle colic artery, as injury of this vessel probably will result in gangrene of a portion of the bowel. It has been stated by G. Feuerer, that if the middle colic artery be wounded, the danger of gangrene is obviated by wrapping the bowel round with omentum. Personally, I would hesitate to trust to this. It is better to take no risks. Wound of the middle colic artery indicates resection of the transverse colon. If resection of the transverse colon be necessary, I think it is simplest to perform a lateral anti-peristaltic anastomosis. This is easier than an iso-peristaltic one, as there is less difficulty in getting the parts in apposition. In some cases it may be necessary to divide the outer layer of the peritoneum at the splenic and hepatic flexures in order to bring together the two portions of bowel without tension.

(vii) The next step is the dissection of the sub-pyloric group of glands.

The omentum is separated upwards along the inferior border of the duodenum, the gastro-duodenal artery, lying in the groove between the duodenum and the pancreas behind 
the pylorus, being ligatured. The omentum and the glands are raised upwards to the left, remaining attached to the pylorus and greater curvature of the stomach.

(viii) The posterior surface of the first two inches of the duodenum is separated from the pancreas, and a clean pad placed in position behind the duodenum.

(ix) A small pair of clamps is placed on the duodenum at the line of the proposed section, usually 1 to $1 \frac{1}{2}$ inches from the pylorus. A pair of pressure forceps is placed on the duodenum to the pyloric side of the clamp and the bowel divided between them. Both ends of the duodenum are cauterised with the actual cautery. The forceps holding the pyloric end of the duodenum is turned over to the right, and the cut surface of the duodenum is covered with a piece of gauze wrung out in warm saline solution.

The distal end of the duodenum is sutured with a continuous suture of catgut, passed right through the duodenum immediately below the forceps, and then over the forceps until the suture has been completed. The clamp is slipped off and the two ends of the suture tied together and cut short, thus closing completely the open end of the duodenum. Round the stump a purse-string suture of linen thread is passed. The end of the duodenum is now invaginated and the purse-string suture tied and cut short. Over this suture a single Halsted stitch is passed. After this stitch has been tied the needle is passed through the sheath of the pancreas, and another knot is made. Thus the stump is buried against the head of the pancreas, a manouvre suggested by Dr. W. Meyer. The blind end of the duodenum closed in this manner rarely leaks. Still it does so occasionally, and as I prefer to take no risks, I leave a small drainage tube from the upper end of the wound down to the duodenal section. In my early cases I did not drain. In two of them leakage occurred, but fortunately the duodenal contents found their 
Plate XVIII.

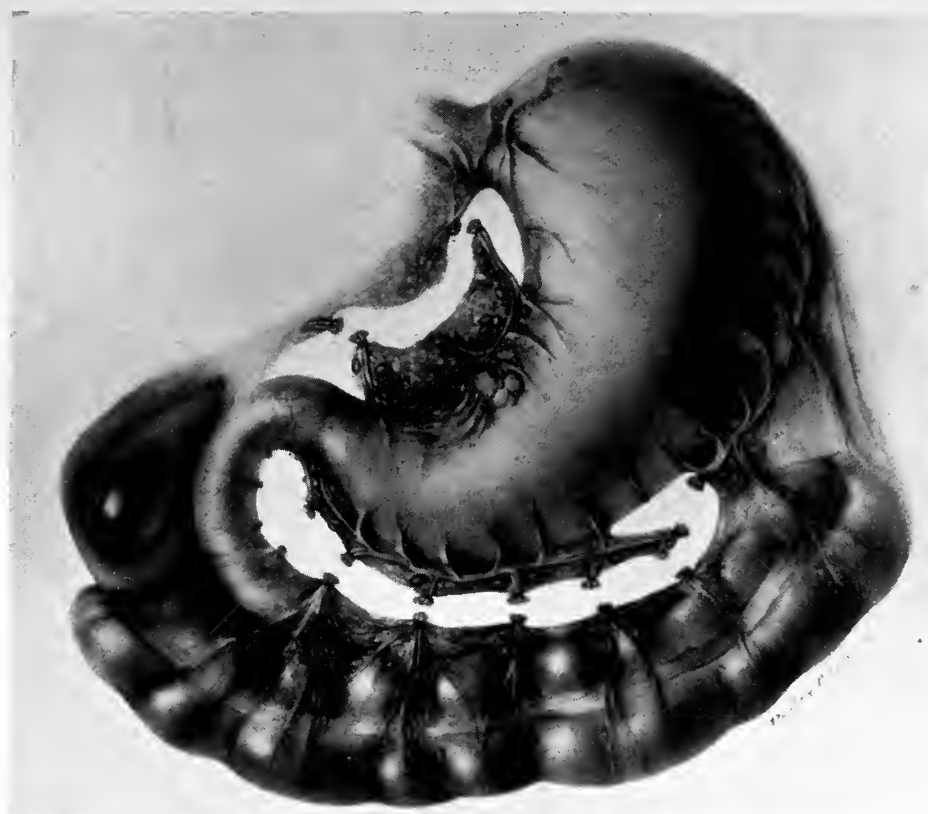

Fig. 40.-Portion of stomach usually resected, showing the dissection of the glands. (W. J. Mayo.)

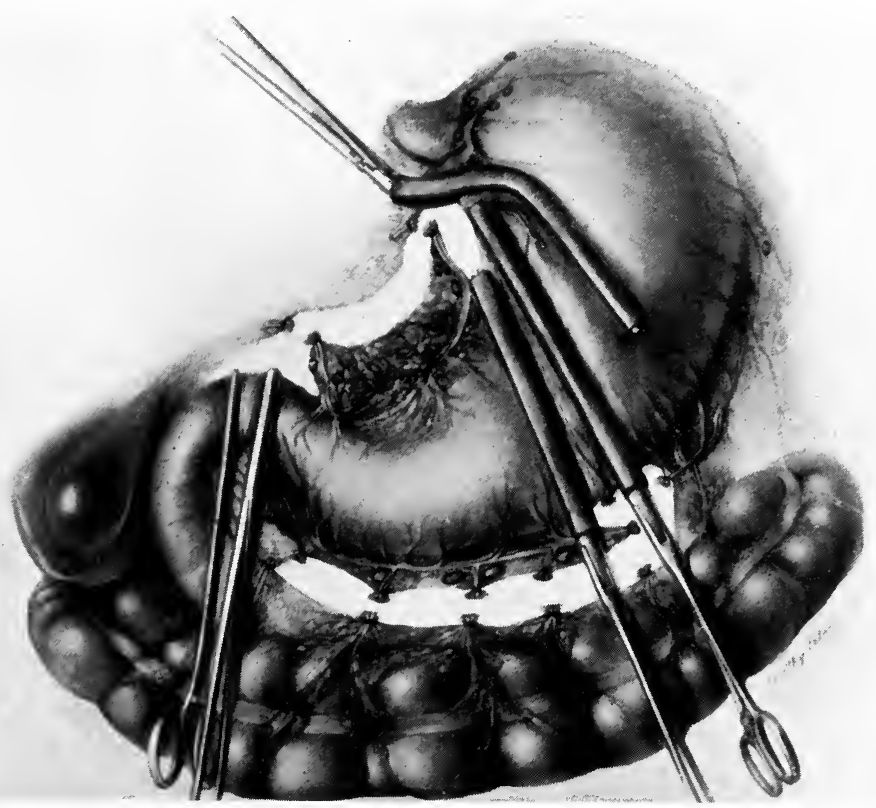

FIG. 41.-Clamps placed on stomach and duodenum isolating pyloric end and lesser curvature. (W. J. Mayo.) 


\section{OPERATIONS ON THE STOMACH}

way through the upper part of the wound, and both patients recovered.

(x) The stomach is drawn downwards and to the right, and a long pair of clamps is placed on it half an inch to the cardiac side of the line of proposed section. Usually it is recommended that clamps covered with rubber tubing should be used. I prefer naked clamps, as they are not so apt to slip, and if not pressed too tightly, they have no injurious effect on the gastric tissue. A second pair of clamps is placed on the stomach distal to the first pair and at an interval of half an inch. A small pair of Porrier's forceps is placed close to the first clamp on its distal side at the lesser curvature, and a second pair at the greater curvature.

These forceps are to prevent the possibility of retraction of the stomach from the clamp when the section is made.

The stomach is now divided with a knife close to the distal pair of clamps, and both cut surfaces are cauterised with the actual cautery. The cut surface of the portion of the stomach to be removed is covered with moist gauze. Commencing at the lesser curvature, a continuous suture of linen thread is passed through all coats of the stomach. This suture should be passed at intervals of about three-sixteenths of an inch, and must be pulled tightly so as to arrest all bleeding.

Before the ends are cut short, the clamp is removed and the cut surface inspected to see if there be any vessels which require ligation.

Another clamp, covered with rubber, is placed on the stomach at a distance of one inch from the suture line. This is used to steady the stomach while the peritoneal suture is being passed. It is most important to secure complete inversion of the cut end of the stomach at the lesser curvature and at the greater curvature. This is accomplished with greater certainty if the peritoneal suture 
be commenced, not at either of the ends, but at the middle of the remaining portion of stomach.

Therefore, I commence the peritoneal suture at the middle of the stomach. This suture is of linen thread, and is passed by the Cushing method, and inverts completely the cut mucous surfaces. If there be much tension, an additional Halsted mattress suture at each end of the suture line gives greater security. All soiled pads are now removed.

(xi) The portion of the stomach which has been separated is now turned over to the right, so that any posterior adhesions may be dealt with deliberately under direct inspection, instead of by touch alone, as would be the case if they were dealt with at an earlier stage of the operation. If the growth be adherent to the pancreas, it is better to shave off a piece of the pancreas, rather than to separate it by tearing. If the pancreas be injured, the raw surface should be covered over with peritoneum as completely as possible, and of course in such a case drainage is imperative.

The retro-pyloric glands are dissected off so that they remain attached to the stomach.

The growth and attached stomach and duodenum are now taken away, all pads removed, and the operation area sponged dry. The gastro-jejunostomy should be inspected, as possibly the resection may render it advisable to correct a kink, by the insertion of an additional suture.

(xii) The abdominal wound is closed in the usual manner, a drain to the blind end of the duodenum being brought out at the upper angle of the wound.

The steps of the operation may be recapitulated as follows:

(i) Making an opening into the gastro-hepatic omentum in order to make an exploration of the growth.

(ii) The performance of gastro-jejunostomy.

(iii) The ligation of the gastric artery. 
Plate XIX.

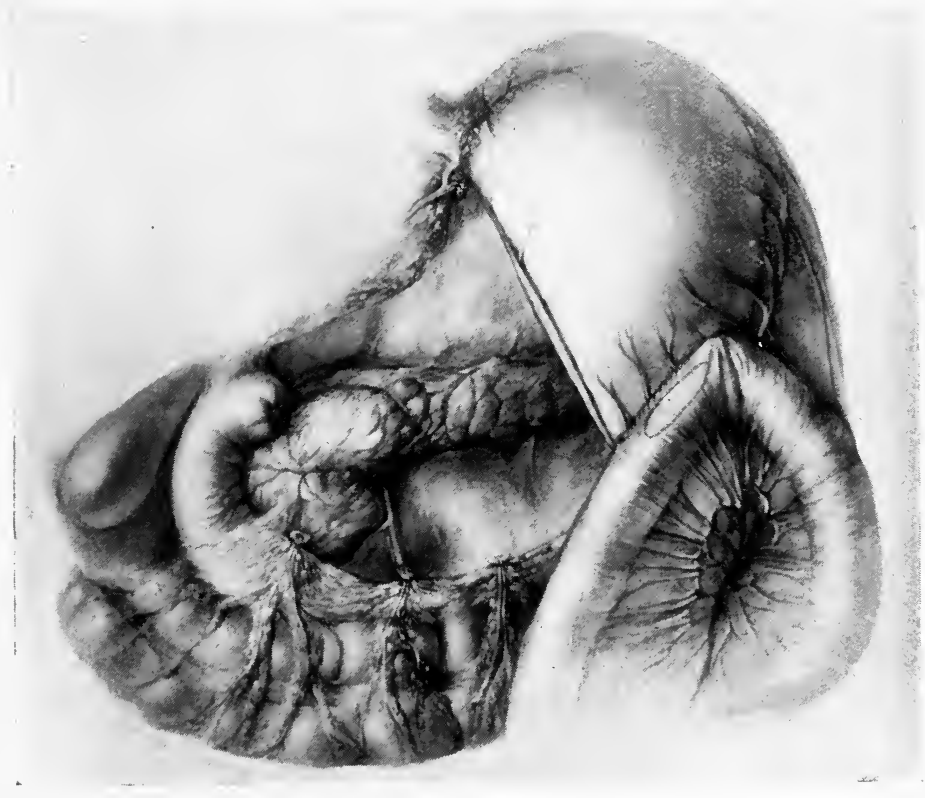

FIG. 42.-Restoration of intestinal continuily by an anterior gastro-jejunostomy. (W. J. Mayo.) 


\section{OPERATIONS ON THE STOMACH}

(iv) The ligation of the pyloric artery.

(v) Dissection of the gastro-hepatic glands from off the stomach at the pyloric and cardiac end of the lesser curvature.

(vi) The ligation of the left gastro-epiploic vessels and of the gastro-colic omentum.

(vii) The dissection of the sub-pyloric group of glands, and freeing of the inferior border of the duodenum.

(viii) The freeing of the posterior surface of the duodenum.

(ix) Division and suture of the duodenum.

(x) Division and suture of the stomach.

(xi) Separation of the posterior adhesions of the growth.

(xii) Closure of the abdominal wound.

The important points are :

1. The use of carefully placed pads to prevent contamination of the peritoneum.

2. The use of clamps with a similar object.

3. Preliminary ligation of the blood-vessels to prevent loss of blood.

4. Complete dissection and removal of all infected glands in one mass, with the portion of stomach removed.

5 . Disinfection with the cautery and perfect invagination of all mucous surfaces.

(b) Complete Gastrectomy.-Occasionally, a case is met with in which, although the whole stomach is infiltrated with cancer, there is an absence of metastatatic deposits and of extensive glandular infection. In such cases the whole stomach may be removed, and the jejunum united to the œsophagus.

The technique is similar to that of partial gastrectomy, except that the jejunum is anastomosed to the anterior wall of the œsophagus instead of to the stomach. The anasto- 
mosis is facilitated by passing a large-sized œsophageal tube from the mouth into the stomach.

\section{EXCISION OF GASTRIC ULCERS}

The method to be adopted varies with the position of the ulcer. In ulcers near the pylorus, the affected portion is excised, the cut ends of the duodenum and stomach closed by suture, and the continuity of the alimentary canal restored by the performance of gastro-jejunostomy. This treatment of calloused ulcers near the pylorus is known as Rodman's operation. The procedure is the same as that described under partial gastrectomy, except that the operation is much less extensive, the excision being limited to the ulcer area.

In ulcers of the anterior wall, the stomach is clamped on either side of the ulcer, two small holes being made into the gastro-colic omentum for the passage of the clamps ; an incision is made in healthy stomach wall encircling the ulcer, which is then cut out, and the gap left is sutured by means of two continuous sutures, an inner including all coats of the stomach and an outer serous suture. The abdomen is closed without drainage.

In saddle ulcers of the lesser curvature a V-shaped incision, having its base at the lesser curvature, is made through the gastric wall so as to include the ulcer and a small margin of healthy stomach. As a rule, a similar incision has to be made on the corresponding part of the posterior gastric wall, a hole being made in the gastro-hepatic omentum in order to expose the posterior aspect of the stomach. The edges of the stomach are sutured in the usual way.

Excision has been practised for hour-glass stomach. Clamps are applied on each side of the constriction. The whole of the portion of the stomach which is the seat of induration is cut away, and the two halves of the stomach united by two continuous sutures. It is advisable in these 
, 


\section{Plate XX.}

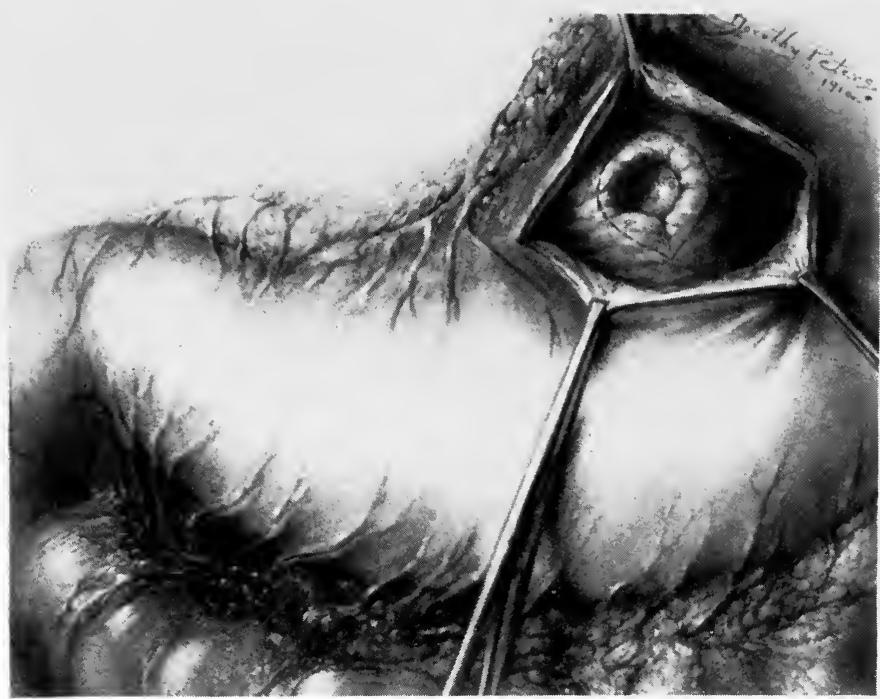

FiG. 43.-Trans-gastric excision of calloused ulcer of the posterior wall of the body of the stomach. The anterior wall of the stomach is opened and the ulcer exposed. The dotted lines show site of proposed excision. (W. J. Mayo.)

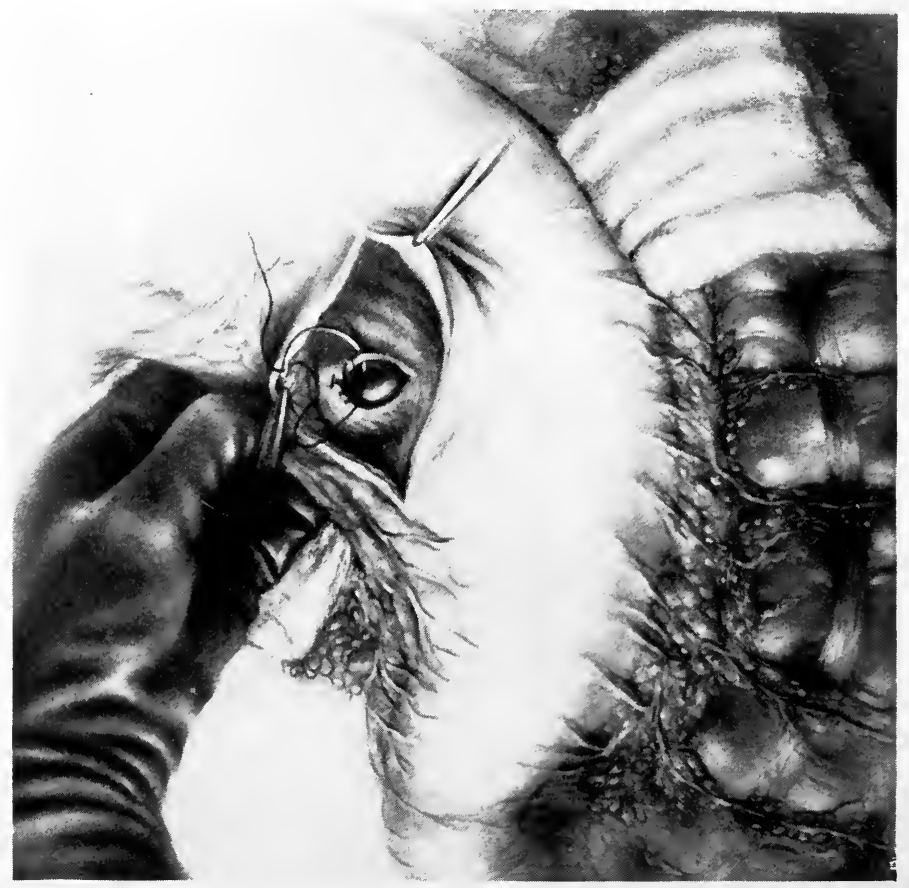

FIG. 44.-Ulcer excised and through-and-through mucous suture partially completed. (W. J. Mayo.) 
cases to pass a tube into the lesser sac for drainage in case of leakage.

For ulcers of the posterior gastric wall, trans-gastric resection was suggested and first performed by Dr. Lewis Pilcher. The method has been advocated by Dr. W. J. Mayo. The steps of the operation are described by Dr. W. J. Mayo as follows :

"The gastro-hepatic and gastro-colic omenta are opened above and below the ulcer. Gauze protection is introduced, adhesions are carefully separated, and if possible, the ulcer surface is cut free from the posterior attachments without opening the stomach. A piece of gauze is packed into the denuded area behind, and in all but one of our cases this temporary pack was adequate to stop hæmorrhage without the ligation of vessels. The anterior wall of the stomach is opened, and with the fingers behind, the entire ulcerated surface is pressed through the anterior incision and the ulcer excised. The gap is sutured with through-and-through sutures of chromic catgut from the mucous side transversely, and this suture line is further protected by several mattress sutures of linen, applied from the mucous side to prevent separation due to the early absorption of the catgut. The anterior wall of the stomach is then closed. Several rubbertissue drains are carried down behind the stomach and brought out at the upper end of the abdominal wound as a safeguard."

\section{JEJUNOSTOMY}

Indications :

(i) Pyloric stenosis, with extreme infiltration of the stomach by cancer, and glandular infection rendering the case unsuitable for resection, or for the performance of gastrostomy or gastro-jejunostomy.

(ii) Cicatrical contraction of the stomach due to the action of strong acids or alkalis. 
The operation rarely is indicated, and seldom is justifiable. When the patient is in such a condition that this operation is the only surgical treatment possible, it is scarcely worth doing. Life can be prolonged only for a few weeks. It is better to give the patient morphia and make the end as comfortable as possible.

When, however, for special reasons, such prolongation of life is highly important, the operation may be done. The best modification of the operation is that of Mr. Mayo Robson. The following is his description :

"The operation consists in taking a loop of the beginning of the jejunum just sufficiently long to reach the surface without tension; the two arms of the loop are short-circuited about three or four inches from the surface, the short circuiting being done either by means of sutures around a decalcified bone bobbin, or by suture alone; personally I prefer the former.

"A small incision is then made into the top of the loop just large enough to admit a No. 12 Jaques catheter, which is inserted and passed for three inches down the distal arm of the loop; this is fixed to the margin of the incision in the gut by a silk or Pagenstecher suture, and the entrance of the tube into the bowel is further guarded by two pursestring sutures, one over the other. The top of the loop is fixed to the skin by one or two stitches and the wound closed. The patient can then be fed at once with some peptonised milk and brandy. The whole operation can be done in from fifteen to twenty minutes, and with very little visceral exposure.

"Should the patient be too ill to bear the little extra time occupied by the short circuiting, the tube may be inserted as directed, and surrounded by two or three pursestring sutures, a proceeding which can be accomplished in a few minutes. In this case the loop of the bowel must not 
be brought to the skin, but had better be fixed by sutures to the peritoneal margin and the aponeurosis, in order to leave part of the lumen of the attached loop within the abdomen for direct passage onwards of the intestinal fluid with the bile and pancreatic secretion."

\section{GASTROPEXY}

Gastropexy has been practised for the relief of gastroptosis. The operation consists in raising the dropped stomach and fixing it higher up in the abdomen. Various methods have been adopted. Thus, Duret fixed the lesser curvature to the anterior parietal peritoneum. Rovsing fixed a considerable portion of the stomach to the anterior abdominal wall, by passing several sutures transversely through the outer coat of the stomach, then passing the sutures through the abdominal wall and tying them over a glass rod. Dr. Coffey supports the stomach by suturing the omentum along the greater curvature to the abdominal wall above the umbilicus. Dr. Beyea raises the stomach by plicating, and so shortening the gastro-hepatic and gastrophrenic ligaments by three rows of interrupted sutures.

Probably the best method is the modification of Dr. Beyea's operation, suggested by Sir Frederic Eve. It is described thus :

"A sandbag is placed beneath the lower part of the thorax as for operations on the liver ; the abdomen is opened to the left of the middle line, and the liver well raised up and held by an assistant, the lesser omentum being thus fully exposed. If the thorax is very long and narrow a headlamp is advisable. The stomach is suspended by four or five interrupted silk sutures passed above and through the upper part of the gastro-hepatic omentum, and below through the lesser curvature.

"Advantage is taken of the fact that the gastro-hepatic 
omentum close to its attachment to the diaphragm and transverse fissure of the liver is much thicker than lower down near its attachment to the stomach; but if the whole membrane is equally thin the sutures are passed above through the liver substance itself, just anterior to the transverse fissure. This is rarely necessary. Below, the sutures are carried through the serous and muscular coats of the stomach just in front of the attachment of the lesser omentum, the vessels, of course, being avoided. The suture furthest on the right is not placed quite up to the pylorus, in order that when the sutures are tied the pyloric end of the lesser curvature may be on a slightly lower level than the portion on the cardiac side.

"A very long needle-holder is required in passing the sutures above under the diaphragm."

It is very doubtful whether any operation for gastroptosis is of real value. Personally, I am strongly against operating in such cases. The patients are seldom better for it, and are often worse.

\section{REFERENCES}

Gastrotomy for Foreign Bodies

Bull, New York Med. Jour., Oct. 29, 1897.

Cathelin, Bull. de la Soc. Anatom., Jan. 1902.

Credé, Archiv f. Klin. Chir., 1886.

Edmunds, F., The Lancet, Feb. 23, 1901.

Ericker, Deutsche Med. Woch., 1897.

Friedenwald and Rosenthal, New York Med. Jour., July 18, 1903.

Heydenreich, La Semaine Med., 1891.

Kooy, Ker., Zeit. f. Klin. Med., 1888, xiv. p. 203.

Paterson, H. J., Clin. Soc. Trans., xxxvii. 1904, p. 215.

Paton, E. P., The Lancet, 1902, i. p. 163.

Richardson, M. H., Boston Med. Jour., Dec. 16, 1886.

Robson, Mayo, The Lancet, 1894, ii. p. 1028.

Schopf, Wiener Med. Woch., Nov. 16, 1899.

Schreiber and Eiselsberg, Mitt. a. d. Grenzgeb. d. Med. u. Chir., 1896, i. p. 729 .

Spencer, W. G., The Lancet, 1900, ii. p. 1423. 
Gastrostomy

Depage, Jour. de Chir., Nov. 1901.

Frank, Wiener Klin. Woch., 1893.

Kader, Cent. f. Chir., 1896.

Senn, Jour. Amer. Med. Assoc., 1896.

Witzel, Cent. f. Chir., 1891.

\section{PyLOROPLASTy}

Berg, Brit. Med. Jour., 1900, ii. p. 1000.

Carle and Fantino, Archiv f. Klin. Chir., xlvi. S. 1, 2.

Caujole, Thèse de Lyon, 1895, No. 1134.

Czerny, Archiv f. Klin. Chir., lvii. p. 461.

Koeppelin, Lyon Médicale, Sept. 24, 1899.

Morison, Rutherford, Brit. Med. Jour., 1905, p. 778.

\section{Pyloro-gastro-Duodenostomy}

Finney, Med. Record, Mar. 7, 1903.

Finney, Brit. Med. Jour., 1905, ii. p. 773.

Mayo, W. J., Annals of Surgery, Nov. 1905, p. 643.

Mayo, W. J., Annals of Surgery, June 1908.

Moynihan, The Lancet, July 29, 1905.

\section{HISTORICAL BIBLIOGRAPHY}

\section{The Technique of Gastro-Jejunostomy}

Wölfler, "Original Operation," Centralbl. f. Chir., No. 45, p. 705 . 1881

Wölfler, "Valvular Opening," Twelfth German Surgical Congress . 1883

Wölfler, "Constriction of Afferent Limb by Silk Ligature," Twelfth German Surgical Congress . . . . . . $\quad . \quad . \quad . \quad . \quad .1883$

Courvoisier, "Posterior Operation," Centralbl. f. Chir., No. 49, p. 794

Wölfler, "Anterior "en-Y,", Verhand. der Deutsche Gesselschaft f. Chir., p. 22 . $\quad . \quad$. $\quad . \quad . \quad . \quad . \quad . \quad . \quad$.

Rydygier, "Anterior with Suture of Efferent Limb to Stomach,"

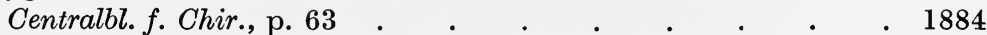

Socin, "Transverse Opening in Gut," Correspbl. Schweiz Aertze . 1884

Von Hacker, "Posterior Operation," Archiv f. Klin. Chir., xxxii. p. 616 . . . . . . . . . . . . . . 1885

Rochwitz, "Isoperistalsis," Deutsche Zeitschr. f. Chir., xxv. p. 555 . 1887

Senn, "Use of Bone Plates," Transactions Ninth International Medical Congress, i. p. 435, and Annals of Surgery, vii. pp.

1, 99, \&c. . . . . . . . . . . .

Robinson, "Raw-hide Plates," New York Med. Journ., p. 429, Oct. 18

Knie, "Operation in Two Stages," Verhandlungen der International Medicinische Congress, ii., section vii., p. 86 . . . .

McGraw, "Use of Elastic Ligature," Journ. Amer. Med. Assoc., xvi. p. 685 
V. Hacker, "Infolding, and Constriction of Afferent Limb," Wien. Klin. Woch., v. No. 48

Maydl, "Anterior "en-Y," Wiener Med. Woch., Nos. 18, $19{ }^{\circ}$.

Maunsell, "New Method of Suture," Amer. Journ. Med. Science, i. p. 245

Von Baracz, "Plates made of Turnip," Archiv f. Klin. Chir., xliv. p. 580

Murphy, "Anastomosis with Button," New York Med. Record, ii. p. 665 .

Brenner, "Anterior Retrocolic," Wiener Klin. Woch., p. 375 •"

Bramam, "Anterior Retrocolic," Centralbl.f. Chir., p. 101 . .

Braun, "Entero-anastomosis between Efferent and Afferent Loops," Centralbl. f. Chir., p. 102

Postinkow, "In Two Stages with Silk Ligature," Centralbl. f. Chir., xix. p. 1018

Haasler, "Anterior Retrocolic," Archiv f. Klin. Chir., xlv. p. 201 . Jaboulay, "Entero-anastomosis between Afferent and Efferent Loops," Archiv Provinc. de Chir., i. p. 429

D'Ursi, "Excision of Great Omentum," Centralbl.f. Chir., p. 448 . McGraw, " "Flap' Incision," Annals of Surgery, xviii. p. 313 . . Robson, "Bone Bobbin," Brit. Med. Journ., i. p. 688 . . . Chaput, "H-shaped Incision," Presse Médicale, July 14 .. . Bastianelli, "Operation in Two Stages," Riforma Medica, iii. p. 506 Sonnenburg, "Valvular Opening (Schrötter)," Deutsche Zeitschr. f. Chir., xxxviii. p. 296

Lauenstein, "Entero-anastomosis inadvisable," Deutsche Zeitschr. f. Chir., xxiv., Nos. 3,4 .

Souligoux, "Operation in Two Stages with Angiotribe," La Semaine Médicale, p. 283

Chaput, "Operation in Two Stages," Bulletins et Mémoires de la Soc. de Chir., p. 602, July 15

Roux, "Posterior "en-Y,", Rev. de Gynécol. et de Chir. Abd., No. i. p. 67

Kocher, "Valvular Opening," Chirurgische Operationslehre . .

Faure, "Invagination of Stomach into Loop," Eleventh Congress of French Surgeons, p. 421

Bouri, "In Two Stages with Caustics," Clinica Chirurgica, p. 290 . Faure, "Modification of Previous Method," Rev. de Gynécol. et de Chir. Abd., ii. p. 81

Paul, "Operation in Two Stages," Brit. Med. Journ., i. p. 439 . Carle, "Sutures with Murphy's Button," Archiv f. Klin. Chir., lvi. p. 55

Sykoff, Archiv f. Klin. Chir., lvi. p. 421 .

Sokoloff, "Uncertainty of Crucial Ligature," Centralbl. f. Chir., p. 590

Modlinski, "Operation in Two Stages," Centralbl. f. Chir., p. 102 Credé, " Perforated Silver Plates," Centralbl.f. Chir., p. 65 


\section{OPERATIONS ON THE STOMACH}

Hildebrand, "Modification of Murphy's Button," Centralbl. f. Chir.,

p. 657 . . . . . . . . . . . 1900

Petersen, "No Loop' Method," Beit. z. Klin. Chir., xxix. p. 597 . 1901

Hall, "Supracolic Route," Lancet, ii. p. 657 . . . . . 1902

Mayo, "Omental Cuff," Boston Med. and Surg. Journ., p. 456, May 1902

Mayo, " Occlusion of Patent Pylorus," Annals of Surgery, p. 44, July 1903

Mikulicz, " No Loop' Method and Transverse Incision of Jejunum," Annals of Surgery; p. 42, July . . . . . . . . 1903

Mayo, W. J., "Direction of Jejunum," Annals of Surgery, p. 537, April . . . . . . . . . . . . . 1906

Mayo, W. J., " Relation of Mesocolic Band," Annals of Surgery, Jan. 1908

\section{Gastroplasty and Gastro-gastrostomy}

Bardeleben, Report by Klemperer, Berlin. Klin. Woch, 1889, p. 221.

Watson, Annals of Surgery, July 1900, p. 85.

Wölfler, Beit. z. Klin. Chir., 1895, No. 13.

\section{Partial Gastrectomy}

Billroth, Deutsche Gesselsch. f. Chir., 1885, Fourteenth Congress.

Feuerer, G., Deutsch. Zeit. f. Chir., 1912, cxvi.

Franke, Centralblatt f. Chir., 1897 ; Beil, p. 98.

Kocher, Op. Surgery (translated by H. J. Stiles), 1903, p. 264.

Krönlein, Beit. z. Klin. Chir., 1896, xvii. p. 93.

Kummel, Archiv f. Klin. Chir., liii. p. 87.

Mayo, W. J., Jour. Amer. Med. Assoc., 1910, liv. p. 1608.

Moynihan, B. G. A., Abdominal Operations, 1906, p. 264.

Paterson, H. J., Gastric Surgery, 1906, p. 102.

Quénu, Rev. de Chir., 1895, p. 850.

Ribera, Revista de Med. y Chir. Pract, April 21, 1903.

Tupolske, Phil. Med. News, March 1893, p. 263.

\section{Total Gastrectomy}

Von Bardeleben, Deut. Med. Woch., 1901, No. 15.

Bockel, Bull. de l'Acad. de Med., Jan. 8, 1901.

Brooks-Brigham, Boston Med. and Surg. Jour., 1898, cxxxviii. p. 415.

Connor, quoted by Hemmeter, New York Med. Rec., 1898, p. 409.

Fedoroff, Russische Med. Rundschau, i., 1903.

Gallet, Soc. Roy. des Sc. Méd. et Anat. de Brux., 58th year, p. 52.

Harvie, J. B., Annals of Surgery, 1900, xxxi. p. 344.

Herczel, Beit. z. Klin. Chir., Bd. xxxiv.

Koble, New York Med. Jour., 1898, ii. p. 125.

Kocher, Th., Deut. Med. Woch., 1897.

Macdonald, Jour. Amer. Med. Assoc., 1898, p. 338.

Moynihan, Brit. Med. Jour., 1903, ii. p. 1458.

Pauchet, Bull. de la Soc. de Chir., June 20, 1900.

Richardson, M., Boston Med. and Surg. Jour., 1898, p. 381.

Schlatter, Lancet, 1898, i. p. 1314. 


\section{Excision of Gastric UlCERS}

Deansley, Brit. Med. Jour., 1910, ii. p. 956.

Dobson, J. F., Brit. Med. Jour., 1912, ii. p. 864.

Mayo, W. J., Annals of Surgery, Dec. 1910.

Pilcher, Lewis, Long Island Med. Jour., 1907.

\section{JeJunostomy}

Moynihan, Brit. Med. Jour., June 1902.

Robson, Mayo, Trans. Roy. Med. Chir. Soc., 1905, p. 236.

\section{Gastropexy}

Beyea, Phil. Med. Jour., 1903, p. 257.

Coffey, Phil. Med. Jour., Oct. 11, 1902.

Davis, Western Med. Review, 1897.

Duret, Revue de Chir., 1896, p. 430.

Eve, Brit. Med. Jour., April 7, 1906, and 1910, i. p. 1101.

Laur, Presse Méd. Belge., 1901.

Ranshoff, Jos., Surg. Gynoec. and Obstet., July 1912, p. 21.

Rovsing, Sammlung. Klin. Vortrage Chir., No. 120, 1906. 


\section{CHAPTER IV}

\section{THE TREATMENT OF PATIENTS AFTER GASTRIC OPERATIONS}

WHEN the operation is completed, the patient should be carried back to bed with the utmost care and gentleness. Sudden lifting or jolting not only puts an unnecessary strain on the wound, but may cause the patient to vomit. The bed should be warmed thoroughly by means of hot-water bottles, but these should be removed, except one for the feet, before the patient is put back to bed. It is desirable to keep the patient warm, but not to cause perspiration. The hot-water bottles should be of stone or indiarubber, and should be enclosed in a flannel bag. The tin hot-water bottles used so commonly in hospitals are very undesirable, and sometimes cause severe and troublesome burns.

Position. - The patient should be propped up in the sitting position (Fowler's position) by means of half a dozen pillows, or a bed-rest. The best arrangement that I know of for preventing the patient from slipping down in the bed, is that devised by Sister Agnes of King Edward VIIth's Hospital for Officers. It consists of a board made of hard wood, $\frac{3}{4}$ inch thick, $6 \frac{1}{2}$ inches wide, and of a length corresponding to the width of the bed on which it is to be used. At each end of this board is fixed an iron bracket, from which projects a short pin which fits into a hole drilled in the side rails of the bed. The brackets are made so that when the pins are fitted into the side rail of the bed, the board lies across the mattress inclined at an angle of $45^{\circ}$ (Fig. 45). The patient's thighs rest against the board, on which a pillow 
is placed for greater comfort. This simple contrivance can be made at a comparatively trifling cost, and is most effectual in maintaining the patient in the desired position.

In an emergency, a simple but less efficient " bed stop" may be improvised in the following manner : A stout bolster (about nine inches in diameter) is placed under the patient's

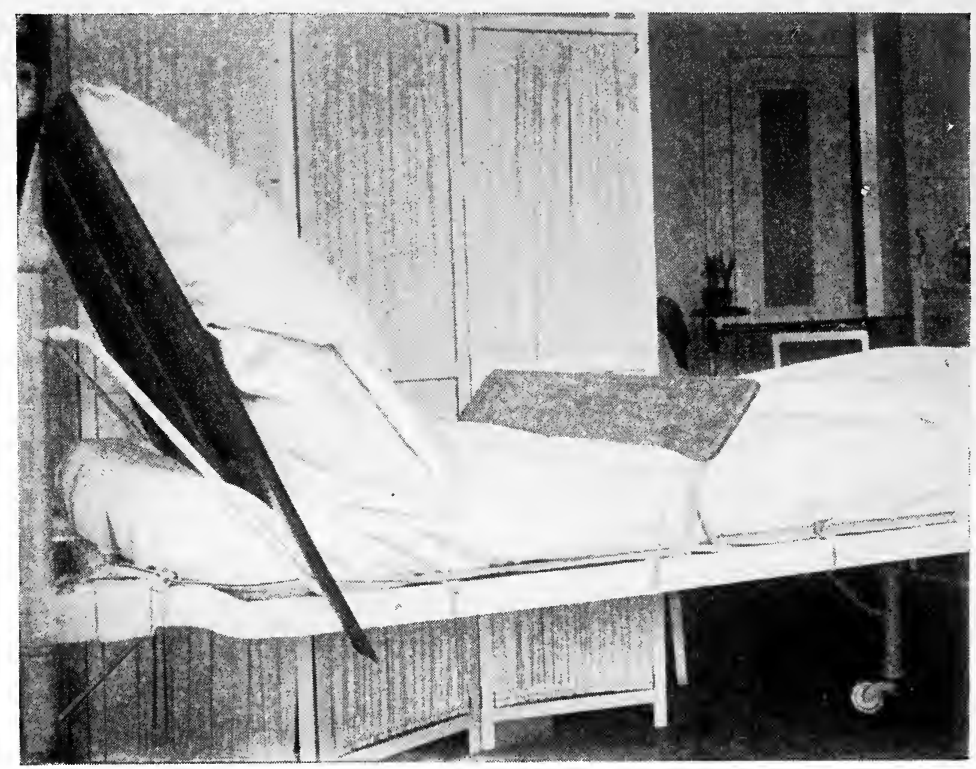

Frg. 45.-Sister Agnes' " Bed-Stop."

thighs. To each end of the bolster a piece of stout webbing is fixed. By tying the other ends of the webbing to the head of the bed, the bolster is fixed, so that it prevents the paitient from slipping. The bolster is more efficacious if it be stiffened by passing a broomstick through its centre from end to end. The webbing in this case is fixed to the ends of the broomstick instead of to the bolster. The disadvantage of this method is that the webbing stretches, and so allows the patient to slip down. To some extent 


\section{TREATMENT AFTER GASTRIC OPERATIONS 63}

this drawback may be obviated by using leather straps instead of webbing to fix the bolster. If webbing straps be used, the arrangement at best is a makeshift.

At night the patient may be turned partially on one side, and after three or four days may, if preferred, lie down on one or other side during the night. I think the Fowler position is the best, after all abdominal operations, but is indicated especially in cases of septic peritonitis and suppurative appendicitis, because it allows free fluid in the abdominal cavity to gravitate to the pelvis, and in suppurative cases pus is thus less likely to track up towards the diaphragm. This is an important matter, for it has been shown that the upper half of the abdomen is much more susceptible to micro-organisms than the lower half. To a considerable extent, the pelvic peritoneum can take up and destroy micro-organisms, but if they reach the area round the liver they become absorbed uninjured, and may cause pleurisy, empyema, and even pyæmia. If there be severe shock, it may be advisable to keep the patient lying flat for a few hours, but in all septic and suppurative cases, I have the patient propped up at once, whether shock be present or not.

Continuous Proctoclysis.-For the first forty-eight hours all my abdominal cases have continuous saline injections by the rectum. This procedure is indicated especially in cases of suppurative peritonitis, but I am satisfied that it is of high value as a routine treatment after all abdominal operations. It is commenced as soon as the patient is back in bed. The largest size rubber rectal tube should be used, with a hole at the end and two holes at the side. The turbe should be passed about three inches into the rectum, and should be kept in place by a piece of strapping passed round the thigh, or one of Murphy's self-retaining tips ${ }^{1}$ may be used.

1 Messrs. Allen \& Hanburys have made some of these for me, and they answer very well. 
The rectal tube is connected by means of two feet of large rubber tubing with a douche can containing from four to five pints of saline solution, which is kept at a temperature of $125^{\circ} \mathrm{F}$. The douche can is raised from four to eight inches above the level of the bed, so that the fluid runs slowly into the rectum at the rate of a pint an hour. After $1 \frac{1}{2}$ pints have been given the flow should be stopped, but the rectal

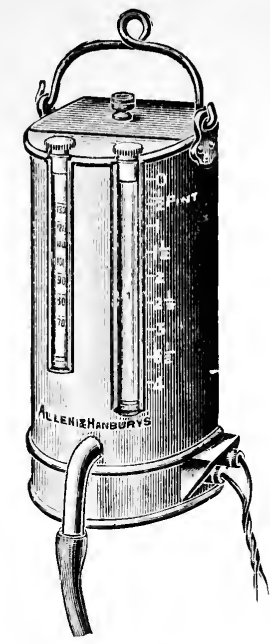

Fig. 46.-The author's electrically heated and if the saline be not retained, generally saline can. should be started again two hours from the time it was originally commenced. After some hours it may be necessary to diminish the rate of flow, or lengthen the interval between the administrations. In this way from ten to fourteen pints can be given in the course of twenty-four hours. The important points are: (1) to keep the temperature of the saline solution constant, and (2) to regulate the flow by gravity, and not by constricting the delivery tube. A feeling of discomfort on the part of the patient indicates that the flow is too rapid, tube should not be removed, and the flow it means, either that the flow is too rapid, or that the temperature is too high, or too low. Dr. Murphy, to whom we are indebted for this valuable method, maintains that if the saline be not retained, it is because it is not given properly. While I am not prepared to deny that a few patients do not retain salines well, I have no doubt that the successful employment of continuous proctoclysis depends mainly on the skill and attention of the nurse. Some nurses are far more successful than others. After an abdominal operation every patient should have the undivided attention of a special nurse for the first thirty-six or forty-eight hours. In a properly 


\section{TREATMENT AFTER GASTRIC OPERATIONS 65}

managed hospital or nursing home, this is arranged as a matter of routine. The regulation of the temperature of the saline requires constant attention, and to get over this difficulty, I have had an apparatus made which simplifies greatly the use of this method.

The apparatus (Fig. 46) consists of an electro-plated douche can which holds about five pints. In the front of the can are a thermometer and a gauge glass, by the side of which the can is graduated in half-pints, so that the amount of saline entering the rectum can be estimated readily. The saline leaves the can through a deliverytube with a $\frac{1}{2}$-inch bore, to which is attached two feet of rubber tubing connected with a large rectal tube. Under the bottom of the can is an electric heater, which can be connected with any electric supply of suitable voltage by means of a flexible cord and wall plug. The can is suspended on an adjustable stand mounted on castors, so that it can

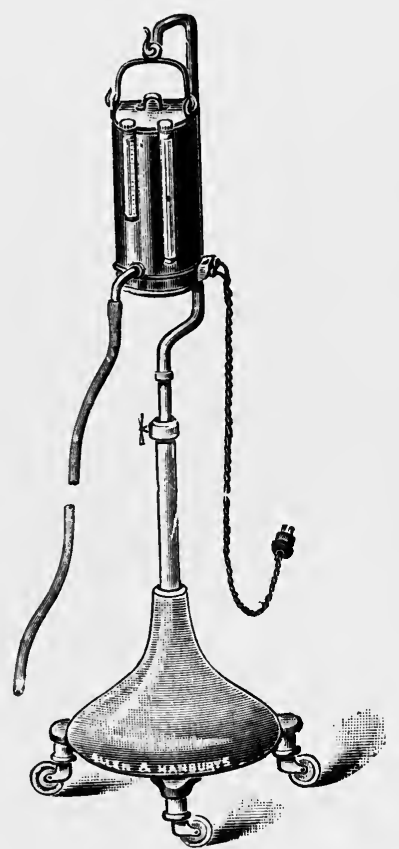
be readily wheeled up to the bedside Frg. 47.-The author's apparatus (Fig. 47). I have found by experifor continuous proctoclysis. ment that with a ward temperature of from $65^{\circ}$ to $70^{\circ} \mathrm{F}$. the solution in the can must be kept at a uniform temperature of $125^{\circ} \mathrm{F}$. in order to ensure that the saline solution enters the rectum at a temperature of from $99^{\circ}$ to $100^{\circ}$. The electric heater is so made, that if the saline solution be put into the can at a temperature of $125^{\circ} \mathrm{F}$. the temperature remains almost constant so long as the current is switched on.

Continuous proctoclysis acts in three ways : (1) It dilutes 
toxins and renders them less harmful. (2) It stimulates the lymphatic flow and so drains the peritoneal cavity. (3) It raises the blood pressure and so diminishes shock. Incidentally thirst is relieved. In cases of septic trouble it should be continued until the patient is out of danger.

Diet.-There is still a lingering superstition that patients must be half-starved after an abdominal operation. A few months ago a surgeon writing on this subject advised nothing but water for twelve hours, and in stomach operations nothing but a little water for three days. I regard such starvation as totally unnecessary, and in old or feeble patients positively harmful. Even after operations on the stomach I begin feeding my patients at once. In one of my gastrojejunostomies for pyloric obstruction, I allowed the patient to have two mutton chops and a milk pudding on the third day. He was thoroughly exhausted by months of vomiting, and was ravenously hungry. He thoroughly enjoyed his meal, and was all the better for it. Of course, I do not suggest this as a routine treatment, but I mention the case to emphasize how groundless is the fear of early feeding. If the anastomosis be efficiently performed, the risk of the sutures giving way may be neglected, so far as feeding is concerned.

As soon as the patient desires a drink, small quantities of hot water are given, and if this be retained one-ounce doses of milk diluted with two parts of water. The quantity is increased gradually up to two ounces hourly. I allow a cup of tea the same day as the operation if the patient wish it. After severe operations, and in elderly and feeble patients, I always order an ounce of Sanatogen to be given during the course of the twenty-four hours. I regard this preparation as a valuable restorative in these cases. On the day after the operation, Benger's food and calves' feet jelly are given as well as milk. As soon as the bowels have been opened 
TREATMENT AFTER GASTRIC OPERATIONS 67

the patient is allowed fluid ad libitum, eggs, thin bread and butter (no crusts), and other soft solids, and usually ordinary diet is resumed in a week or ten days.

As a general rule the patient's inclination is a reliable guide to the quantity of food required, although in a few cases some coaxing and diplomacy are necessary to induce the patient to take adequate nourishment. It is impossible to lay down hard-and-fast rules as to feeding, and general rules have to be modified in individual cases. After operation for septic peritonitis, no food is given by the mouth until the bowels have been opened thoroughly. After gastrojejunostomy for gastric or duodenal ulcer, especially if associated with hyper-acidity, the diet must be more limited in quality, although the quantity need not be curtailed. I always urge these patients to keep on a milky diet for at least six months. On the other hand, after gastro-jejunostomy, or partial gastrectomy for cancer, I feed the patients up more rapidly, allowing mutton or beef essence, jelly, eggs, and Benger's food on the second day, and often fish cream or chicken cream on the third day. Patients who have been exhausted by weeks or months of vomiting will not stand starvation, and their tissues possess feeble power of repair, unless they be provided with plenty of nourishing food.

Rectal Feeding.-Rectal feeding is a scientific term for starvation. Formulæ for so-called nutrient enemata composed of eggs, milk, beef-tea, \&c., still survive in text-books and hospital pharmacopœias, relics of the superstitions of the past. I often wonder whether the surgeons who prescribe them have seen or smelt the odoriferous mess which is washed out of the rectum when such concoctions are administered. In my experience rectal feeding is quite unnecessary after abdominal operations, and I have satisfied myself by experiment that little, if indeed any, of the constituents of these mixtures is absorbed. After an ordinary 
abdominal section, usually the patient can take enough nourishment by the mouth, and the fluid required by the body can be administered by the rectum in the manner already described. In cases of septic peritonitis, as a rule, the patients have been in good health up to the time of the onset of the illness, and can do without much nourishment until food can be administered by the mouth. In these patients it is fluid that is required rather than nourishment. In those exceptional cases in which nourishment is necessary, and cannot be given by the mouth, I add dextrose to the saline injection.

The Bowels.-I regard the early opening of the bowels as one of the most important points in the treatment of patients after abdominal operations. No hard-and-fast rules can be laid down as to how soon an aperient should be given. Each case must be dealt with according to the conditions present, and there is no detail of after-treatment in which sound judgment and experience are of such importance. My guiding principle is, that the sooner the bowels are opened, the sooner is the patient out of danger. In an ordinary uncomplicated case I give an aperient usually on the third morning, and in my opinion there is no better aperient than calomel given in half-grain doses every hour until the patient has had three or four grains.

A few hours after the administration of the last dose a turpentine enema is given. If this be ineffectual, three or four drachms of castor-oil are given at night, followed by another enema. In pelvic cases, in cases in which there has been much handling of intestine, or in cases in which there is distension after operation, I commence the administration of calomel on the second day. After operations for septic peritonitis and for perforated gastric or duodenal ulcer (provided I have performed a gastro-jejunostomy as well as sutured the ulcer), I often commence the administration of 
TREATMENT AFTER GASTRIC OPERATIONS 69

calomel the same night as the operation, in some cases as soon as the patient has come round from the anæsthetic. In these cases, in addition to calomel, sometimes I give subcutaneous injections of eserine salicylate, gr. $\frac{1}{100}$ every four hours until five or six doses have been given. When there is distension, as well as vomiting, a good plan is to give five grains of calomel immediately after an attack of vomiting. This may be repeated if necessary. In the case of a woman with extreme distension and vomiting after operation for extra-uterine gestation, nearly twenty grains of calomel were given in this way during twenty-four hours. On the third day the patient's bowels were freely opened, she experienced immediate relief, and thereafter made an uneventful recovery. I have never yet had to regret giving an aperient early.

Treatment of the Wound.-I believe in a tight bandage for the first twelve hours; after this it may be loosened and readjusted. There is nothing so comfortable as a many-tailed bandage made of strips of domette seven inches wide and about seven feet long. The strips should be placed one inch apart, and should not be sewn together behind, as is usual. It adds to the patient's comfort if the bandage be undone and rebandaged every day, without disturbing the dressings. Every two or three days the bandage may be taken off and a fresh one put on. The old one can be washed and ironed ready for the next change. I have my wounds dressed and the skin suture removed on the sixth day. The sutures used to anchor the dressing are removed a few days later.

Drainage Tubes.-Drainage tubes occasionally are left in too long, but far more frequently they are removed too soon. This is especially the case after operations for general suppurative peritonitis, in which it has been necessary to place a drainage tube in Douglas's pouch. Such a tube should be kept in situ for at least a week, and sometimes for longer. If removed earlier it may be impossible to replace it, and as 
a result of too early removal, an abscess may form which may require another operation for the evacuation of the pus. As a general rule a drainage tube should not be removed until any discharge of pus has ceased for three or four days.

The Duration of Rest in Bed.-There is a great diversity of opinion as to how long a patient should be kept in bed after an abdominal section. Personally, I believe in shortening rather than in lengthening this period. My practice varies according to the situation of the wound. After operations for appendicectomy, in which I use the Battle incision, I allow the patient to get on to a couch in a week, if so inclined, and to sit in an armchair two or three days later. When the incision is above the umbilicus, three weeks in bed, and when below the umbilicus a few days longer, are advisable. In operations for cancer I insert some additional through-and-through sutures, and then get the patient into a chair on the fourth or fifth day. Patients suffering from cancer do not bear confinement to bed well, and the sooner they are got up the better. After operation for gastric ulcer in patients who have been losing blood, or who have been on a restricted diet for a long time, the decision as to when it is advisable to allow them to get up needs some discrimination. Often such patients have weak, flabby hearts, and require feeding up, and a course of digitalis and iron, before they are fit for much movement. If they be allowed up too soon, they may die quite suddenly from syncope. In such cases, a subnormal temperature and a slow pulse should be regarded as danger signals. On the other hand, I believe that too long confinement to bed favours thrombosis, with the danger that when the patient gets up an embolus may be detached.

All patients should wear a firm binder or abdominal belt for six months after operation, and before discarding the belt should have abdominal massage for two or three weeks. 


\section{CHAPTER V \\ COMPLICATIONS AFTER GASTRIC OPERATIONS}

1. Shock.-For practical purposes we need not draw a distinction between shock and collapse. The treatment is the same, namely, the treatment of "the condition resulting from a fall in the general blood pressure." The chief causes of shock after an abdominal operation are: 1. A prolonged operation. 2. Hæmorrhage. 3. Exposure or rough handling of viscera.

There is another type of shock due to the absorption of toxins, which is apt to occur after operations for suppurative peritonitis. This is the most serious type of shock, and the most difficult to treat, and I am convinced, contrary to the opinion expressed by some, that washing out the peritoneal cavity greatly increases its severity.

The ideal treatment of shock is preventive. In my judgment by far the most potent means of preventing, or at any rate lessening, shock is the use of morphia and ether instead of chloroform for the production and maintenance of anæsthesia. When ether is given by the open method it is rare for symptoms of shock to be marked, owing to the action of this combination of narcotics in blocking the afferent nerve impulses. I do not say that shock is absent entirely, but that in the cases in which it occurs, its onset is delayed until the effects of the ether and morphia are passing off, and at this stage it is easier to treat shock than during, or just after, the operation.

The first thing to bear in mind in the treatment of shock 
is that strychnine, brandy, and other so-called stimulants are useless, and indeed positively harmful. This has been shown conclusively by the work of Dr. G. W. Crile and others. Strychnine given to a healthy person raises the blood pressure, but when administered to a person already suffering from shock, it causes a marked fall of blood pressure, a result exactly opposite to that required in the treatment of shock. The best treatment for shock is the administration of continuous saline solution by the rectum, by the method described already. As regards drugs, adrenalin causes a marked rise in the blood pressure. A drachm of adrenalin chloride ( 1 in 1000) may be added to each pint of saline solution. The objection to adrenalin is that its effect is very transient, so that to be of service the administration of the drug must be continued. Extract of pituitary body is a much more powerful agent, and its effects are more lasting. In an adult, seventeen minims (1 c.c.) injected into the deltoid muscle causes a marked rise in the blood pressure, which lasts for eight or ten hours. It is, however, a powerful drug, and the dose must be regulated carefully according to the age and strength of the patient. Until shock has passed off, the patient's head should be kept low by raising the foot of the bed. The repeated application to the head and forehead of cloths wrung out in very hot water is a valuable addition to the methods for treating shock which I have mentioned.

I never give intravenous injections of saline solution, as $I$ believe that in some cases they do harm. If there be any difficulty with rectal administration, I prefer to let the saline solution run into the subcutaneous tissues of the breast and axilla. As I have mentioned already, I have all suppurative cases propped up in bed at once, whether shock be present or not.

2. Pain.-I have included pain among the complications 
of abdominal operation, because I believe that pain as distinct from discomfort is the exception rather than the rule. There is no doubt that patients vary very much in their susceptibility to pain, and in their capacity for bearing it. It is rare for hospital patients to complain much of pain after an abdominal operation; private patients, on the other hand, complain much more frequently of pain during the first twenty-four hours. I am very strongly averse from giving morphia after an abdominal operation; it relieves pain, but it is harmful in other ways, and certainly adds to the patient's subsequent discomfort. We should try to ascertain the cause of the pain, and if possible treat such cause. Pain may be due to the patient's position, to undue tightness of the bandage, to excessive tightness of sutures, and in wounds above the umbilicus to the movements of the diaphragm. A frequent cause of pain, especially after the first twenty-four hours, is intestinal colic. This can be relieved at once by a turpentine enema, or a dose of calomel. In such a case morphia is the very worst mode of treatment. I confess that I dislike giving any drugs after an abdominal operation, but if pain be so severe as to require treatment, ten grains of aspirin repeated every six hours up to thirty grains, or a dose of trional, is the least harmful remedy. In neurasthenic patients, the value of an hypodermic injection of sterile water should not be overlooked. Often it acts like a charm.

3. Septic Peritonitis.-It is scarcely an exaggeration to say that septic peritonitis as a sequence of operation in a clean case has been abolished by the methods of modern aseptic surgery. It may occur from leakage owing to faulty application of the sutures of an anastomosis. With a double row of sutures carefully applied, leakage should not occur, and I have not yet met with it. Leakage may occur in rare instances through no fault in technique, but owing to 
feeble repair on the part of the patient. Septic peritonitis may result also from contamination of the peritoneum with intestinal contents at the operation. By the use of clamps and pads such soiling is reduced to a minimum. Thorough preparation of the patient beforehand, on the lines laid down in a previous chapter, greatly reduces the probability that such soiling as occurs will do serious harm. The infection produced by the soiling will be slight, and the peritoneum is able to deal with it safely.

The treatment of septic peritonitis following operations is beset with difficulty. In by far the larger majority of the cases, the safest course is to treat the patient by absolute rest, lavage of the stomach, and ice to the abdomen, in the hope that the peritonitis may be limited. If it be probable that leakage has occurred, the patient's only chance is a second operation, to repair the leak, to cleanse the surrounding parts, and to establish drainage. Such operations rarely are successful. The patient's strength and nervecells have been exhausted already by one operation, and will be depleted still further by another. Therefore, before deciding to reopen the abdomen, the surgeon should have strong grounds for believing that a leakage has occurred. It is only under such circumstances that a second operation is justifiable.

When septic peritonitis is present at the time of the original operation, early opening of the bowels is indicated. In my experience, when once the bowels are thoroughly well opened, the patient may be regarded as out of danger.

Our aim should be to prevent peritonitis; strict asepsis, the use of clamps and pads to prevent the soiling of the peritoneum, and early opening of the bowels after operation, are the chief safeguards against this complication.

4. Post-anæsthetic Vomiting.-When ether by the open method, in conjunction with morphia, is the anæsthetic em- 
ployed, post-anæsthetic vomiting is rare as a complication of abdominal operations. Since this anæsthetic has been the one usually adopted for my patients, I do not think that I have had to treat a single case of post-anæsthetic vomiting. If vomiting, due to the anæsthetic, persist, the best plan is to give a good drink of warm water containing 20 grains of bicarbonate of soda. If this fail the stomach may be washed out. Vomiting is certainly less common when the patient is propped up in the sitting position. Care should be taken that the head be turned to one side by placing a pillow under the opposite shoulder. The persistent vomiting which sometimes follows chloroform narcosis, may be relieved, in some instances, by adding an ounce of glucose to the saline injection. In operations for intestinal obstruction, often it is advisable to wash the stomach out before the anæsthetic is given, and in all cases it is well to do so before the patient is put back to bed after the operation. In cases of vomiting from septic peritonitis, nothing should be given by the mouth, gastric lavage should be repeated at regular intervals, and the administration of calomel and eserine salicylate should be commenced at once in order to secure emptying of the intestinal canal as early as possible. When this has been accomplished usually the vomiting ceases.

5. Pseudo-ileus.-Pseudo-ileus, or paralytic distension of the intestine, is most likely to occur in cases in which there is suppurative peritonitis, or in which there has been much handling, damage, or exposure of the intestines, or in cases in which it has been necessary to pack part of the abdominal cavity with gauze. If calomel be administered early, as I have advised, this complication will not occur often. When it does occur, the treatment is energetic administration of eserine salicylate, calomel, and turpentine enemata. I confess, however, that I am very sceptical as to the muchvaunted efficacy of eserine salicylate. When gauze packing 
has been employed, the packing should be removed as early as possible. Strychnine is strongly recommended by some for the treatment of pseudo-ileus. In my experience it has proved quite valueless.

6. Lung Complications.-Bronchitis and pneumonia follow abdominal operations with far less frequency than formerly was the case. Often they are attributed to the anæsthetic, especially to the chilling and irritating effect of ether vapour on the respiratory passages.

I confess I am very sceptical as to the occurrence of a true ether pneumonia. Mr. Bellamy Gardner has shown that when ether is being given by the open method, the temperature of the vapour under the mask is only one degree below that of the surrounding atmosphere. Dr. W. J. Mayo has told me that in more than one instance he has seen pneumonia follow a gastric operation performed under local anæsthesia.

Dr. W. Pasteur has shown that the incidence of lung complications is greater after chloroform than after ether, the percentage in the case of chloroform being $7 \cdot 74$, and in the case of ether 5·18. Possibly in many instances lung complications have been due directly to the rebreathing of the contaminated air in the bag of a Clover's inhaler, an apparatus which has served a useful purpose in the past, but which, now that we have the open method of ether administration, should be seen only in museums of medical antiquities. I think there can be no doubt that the adoption of the Fowler position after abdominal operations has diminished greatly the frequency of lung complications. Formerly pleurisy and empyema were not infrequent after operations for acute appendicitis. Since the routine use of the Fowler position I do not remember that I have met with either of these complications. Probably in some instances lung complications are due to slight local sepsis resulting in 
the detachment of a small septic embolus. In by far the greater number of cases I believe bronchitis and pneumonia following operation are due to deficient aëration of the bases of the lungs and to the accumulation of secretion in these situations. This hypostatic congestion is the result of the interference with the working of the diaphragm, partly from too tight bandaging, and partly from the involuntary inhibition of its movements caused by the wound.

It is no doubt a truth, based on the observations of many surgeons, that lung complications follow gastric operations more frequently than any others. And it is in stomach operations that the wound in the abdominal wall is situated nearest to the diaphragm.

I feel convinced that the occurrence of pneumonia and bronchitis in my practice has been diminished greatly by encouraging the patient to take deep breaths at regular intervals during the days following operation.

In the prevention of lung complications I attach great importance to keeping the patient warm during the operation, both by means of clean, warm clothing, and by the use of an operation table heated by electricity or other means.

The treatment of the bronchitis and pneumonia after abdominal operations presents no unusual features.

7. Parotitis.-Parotitis may occur in cases in which food by the mouth is withheld. The treatment is preventive, with careful attention to the cleansing of the mouth, and frequent rinsings with listerine or hydrogen peroxide. In addition, the plan adopted by Dr. Soltau Fenwick is a good one. He gives the patient a baby's rubber teat to suck. The constant sucking induces a flow of saliva which helps to keep the mouth clean. The teat should be sterilised frequently by boiling. When parotitis occurs, fomentations should be applied, and if pus form, it should be evacuated in the ordinary way. 
8. Phlebitis and Thrombosis.-Phlebitis and thrombosis are less common after gastric than after pelvic operations. Thrombosis usually is secondary to phlebitis, but occasionally thrombosis is the primary condition. It is more likely to occur in feeble, debilitated patients whose blood current is sluggish. One of my patients died quite suddenly three days after an ovariotomy. At the post-mortem examination an ante-mortem clot was found in the pulmonary artery. Phlebitis is probably septic in origin, and is met with most frequently in the saphena and femoral veins of the left lower limb. It is rare for this complication to occur before the second or third week.

The treatment is absolute rest, and elevation of the affected limb, and the period of confinement to bed should be increased to five or six weeks. Sir Berkeley Moynihan speaks well of the intravenous injections of collargol.

9. Post-operative Hæmatemesis. - Hæmatemesis may occur after operations on the stomach owing to imperfect application of the sutures, so that bleeding ensues from the cut edges of the stomach, or intestine. This may be spoken of as avoidable post-operative hæmorrhage, in distinction from unavoidable post-operative hæmatemesis.

By unavoidable post-operative hæmatemesis is meant, hæmorrhage for which there is no apparent cause. Although many papers have been written on the subject, the ætiology of such hæmorrhage is not clear. Professor Von Eiselsberg considers that it is due to injury of the omentum. This certainly does not explain all cases. Dr. Rodman attributes it to sepsis, an explanation doubtless applicable in some cases, but not in others. It is a very fatal complication, with a mortality rate, according to Dr. Powers, of nearly 70 per cent. I have met with one instance, after separating adhesions around a jejunal ulcer. The patient had considerable hæmatemesis on the second and third days, but recovered. 
The amount of blood vomited at one time is small, but the vomiting is frequently repeated. Surgical intervention is contra-indicated in these cases. The treatment should be ice to the abdomen, gastric lavage, very hot saline by the rectum, and abstinence from food by the mouth and the administration of calomel.

10. Acute Dilatation of the Stomach.-Acute post-operative dilatation of the stomach is a serious complication. In some cases the gastric dilatation is the result of septic peritonitis, and is accompanied by a general distension of the intestines. In other cases the stomach alone is dilated. Why dilatation occurs is not quite clear. Allbrecht suggested that the superior mesenteric artery presses upon and causes obstruction of the third part of the duodenum. This explanation doubtless holds good for some cases. Other writers maintain that the dilatation is the result of a primary paralysis of the musculature of the stomach. Dr. Box and Mr. Wallace suggest that two factors come into play in producing acute gastric dilatation. "First, a paralytic condition of the viscus which leads to distension and then, at a certain stage, the distended stomach actually produces obstruction by pressing on the duodenum on the front and to the left of the spinal column." An excellent account of the subject has been published by Dr. Campbell Thomson.

Repeated vomiting is the prominent symptom, the vomit being brought up in gulps without straining, and at frequent intervals. When, after an abdominal operation, vomiting persists or comes on without apparent reason, the dressings should be undone and the abdomen examined. The bulging of the abdomen above the umbilicus, and retraction below, are very striking in cases with this complication. As to treatment, the stomach should be washed out at intervals, and the patient placed in the knee and elbow position. In the majority of instances, unless the dilatation be due to 
septic peritonitis, these means will suffice. By keeping the stomach empty by lavage, gradually it recovers its tone and is able to empty itself. Acute dilatation may occur after any abdominal operation, especially after operations for perforated gastric or duodenal ulcer; therefore a close watch should be kept for this complication, as sometimes a fatal issue may be prevented by timely lavage.

I have met with this complication four times. In one of them I performed gastro-jejunostomy with a fatal result. I think gastro-jejunostomy is contra-indicated in these cases, as with the existence of gastric atony the short circuit is of little use. In three of my cases acute dilatation followed gastro-jejunostomy; two of them were after operation for perforated duodenal ulcer, and in the third after operation for " trifid stomach." In the latter case the patient vomited profusely for sixteen hours and I was not informed of her condition. When I saw her she was cold and clammy, the pulse almost imperceptible. I drew off nearly five pints of dirty fluid from the stomach. I did not expect her to recover. To my surprise, after being very ill for twenty-four hours, she made an uneventful convalescence.

\section{Complications peculiar to Gastro-jejunostomy}

(a) Immediate.- The condition known as " circulus vitiosus," or regurgitant vomiting, was formerly the most dreaded complication of gastro-jejunostomy. The vomiting was attributed to the reflex of bile into the stomach from the afferent loop of the jejunum. The presence of bile, in the stomach has no injurious effect, provided it can get out again. It is not the presence of the bile, but the obstruction to its leaving the stomach, which causes the vomiting. Various explanations have been given as to the cause of regurgitant vomiting. Some believe that it is due mainly 
to the leaving of a loop of jejunum between the flexure and the anastomosis, and that this loop, becoming "waterlogged," is unable to pass on its contents, or by its weight causes a kink at the site of the anastomosis. Why a piece of healthy intestine should become water-logged, apart from mechanical obstruction, is not explained. There is not, I think, any evidence of such a causation. I believe that most, if not all, cases of regurgitant vomiting are due to mechanical obstruction at the efferent opening.

As Dr. Terrier has remarked: "The establishment of the circulus vitiosus is due to faulty operating." Whether the vomited material regurgitate from the stomach into the afferent loop, and thence back to that organ through the pylorus, is immaterial; the real factor is obstruction at the efferent opening, and when water-logging of the jejunal loop occurs it is secondary to some obstruction.

After the anterior operation, obstruction may be caused by a kink at the point where the efferent limb is attached to the stomach, or by the use of a jejunal loop of insufficient length to allow of its being brought over the transverse colon without tension.

After the posterior operation, I am of opinion that in many of the cases, obstruction is due to accidental twisting of the jejunum on its longitudinal axis during the process of suturing, or to flattening of the jejunum by the inclusion of too much tissue in the sutures.

Dr. W. J. Mayo has pointed out that the normal direction of the jejunum at its origin is from right to left. If, then, the jejunum be attached to the stomach iso-peristaltically, a sharp angle is produced immediately above the anastomosis, which may give rise to trouble.

In the majority of cases gastric lavage for a few days suffices to cure the patient.

Dr. P. Mauclaire states that the knee-elbow position for 
twenty-four hours is sufficient to arrest the symptoms in many instances. If, in spite of these measures, serious vomiting continue, the abdomen must be reopened. If an anterior gastro-jejunostomy has been performed the surgical treatment is very simple; an entero-anastomosis is performed between the afferent and efferent limbs. I have never had occasion to treat a case of acute regurgitant vomiting after the posterior operation, but the performance of an enteroanastomosis in these cases must be a very difficult proceeding.

I believe that some of the slighter cases of regurgitant vomiting, which do not follow operation immediately, are due to simple constipation. One of my own patients returned two months after gastro-jejunostomy, and stated that about once a fortnight she had severe attacks of vomiting. By inquiry I elicited the fact that the attacks invariably were preceded by constipation for several days. The patient was instructed to pay great attention to obtaining daily evacuation of the bowels, and since that time she has had no further trouble. I have observed two other similar instances. Why after gastro-jejunostomy should constipation give rise to vomiting? I think that a simple explanation may be offered. It is probable that when any part of the intestine is loaded with fæces, the onward peristaltic waves are arrested by the distension and temporary paresis of that portion of the gut. In such a case a reflux wave of peristalsis may occur, tending to drive the intestinal contents backwards. Now, in a normal individual, probably the duodenum acts as a syphon-trap, and so checks regurgitation of intestinal contents into the stomach from such slight reflux movements as would be caused by temporary obstruction. This syphon action of the duodenum is further aided by the contraction of the pylorus, so that little or none of the intestinal contents gains entrance to the stomach. After gastro-jejunostomy, however, both the syphon-trap and the 
pyloric sphincter are put out of action, so that there is no obstacle to regurgitation from the efferent loop into the stomach. In this way a degree of constipation, which in a normal individual would cause discomfort only, may produce vomiting in a person upon whom gastro-jejunostomy has been performed. I think that this is the explanation of the so-called " bilious attacks" from which patients suffer sometimes after gastro-jejunostomy.

After gastro-jejunostomy there is always a slight regurgitation of bile into the stomach. In some instances this is excessive. The patient does not vomit, but is uncomfortable, has a feeling of nausea, and suffers from loss of appetite. In some of the cases the fault is one of technique, a slight obstruction at the anastomosis, but not sufficient to cause vomiting. With modern technique this ought not to happen. More often the reason is that gastrojejunostomy has been performed for gastric symptoms in patients who had no gastric lesion. If the patient have some other lesion, such as gall-stones, or appendicitis, gastrojejunostomy will not give relief. If gastro-jejunostomy be performed on a markedly atonic or gastroptosed stomach, the result almost certainly will be a failure. I have opened the abdomen in many instances in which symptoms have persisted after gastro-jejunostomy has been performed elsewhere, and have found no lesion present which rendered the anastomosis necessary. In these cases undoing the gastrojejunostomy and restoring the continuity of the intestinal canal usually has given complete relief. In some of them either a diseased appendix or cholelithiasis was discovered and dealt with.

Diarrhœa.-Cases have been recorded in which death has resulted, apparently from simple diarrhœa. Attention has been called to this complication by Kelling, Anchütz, and by Carle and Fantino, who state that of eleven fatal cases 
of gastro-jejunostomy, in four the cause of death was diarrhœa. I have observed this complication twice, but in neither instance did it cause any alarm. I believe that it is probably a symptom of intestinal toxæmia, and is an indication for the administration of calomel. In my first case, after gastro-jejunostomy for ulcer, it was checked at once by the administration of two doses of calomel and a large turpentine enema. In the other case, after gastro-jejunostomy for advanced carcinoma, it proved more troublesome, but ceased gradually after the patient was given one-sixth of a grain of calomel four times a day.

Internal hernia.-Several instances of internal hernia after gastro-jejunostomy have been recorded. In most of them a portion of small intestine has slipped through the artificial aperture in the mesocolon and has become strangulated.

So far as I am aware, since the adoption of the practice of suturing the edges of the mesocolon to the jejunum or stomach, no instance of this complication has been recorded.

(b) Remote.-The complication of jejunal and gastrojejunal ulcer is dealt with in a later chapter (Chap. VII).

\section{REFERENCES}

\section{Post-operative Complications. Lung Complications}

Bibergeil, Brit. Med. Jour. epit., 1906, i. p. 41.

Graves, W. P., Boston Med. and Surg. Jour., Sept. 29, 1910.

Mayo, W. J., Annals of Surgery, Aug. 1902.

Munro, J. C., Jour. Amer. Med. Assoc., Aug. 7, 1909.

Pasteur, W., The Lancet, May 20, 1911.

Riley, E. S., Boston Med. and Surg. Jour., Jan. 20, 1910.

\section{Phlebitis and Thrombosis}

Cordier, Jour. Amer. Med. Assoc., 1905, ii. p. 1792.

Friedmann, Beit. z. Klin. Chir., Sept. 1910.

Moty, F., Semaine Méd., Mar. 18, 1908.

Moynihan, B. G. A., Abd. Operations, 1906, p. 89. 


\section{Parotitis}

Bucknall, The Lancet, 1905, ii. p. 1158.

Dyball, Annals of Surgery, xl. p. 886.

Paget, S., Trans. Med. Soc., 1887.

Soubeyran and Rives, Archiv génér. de Chir., May 1908.

\section{Post-operative Hamatemesis}

Meyer, W., Surg. Gynoecol. and Obstetrics, Aug. 1911. Purves, R., Edin. Med. Jour., March 1902.

\section{Acute Dilatation of the Stomach}

Box and Wallace, The Lancet, 1901, ii. p. 1260.

Chavannar, Jour. de Méd. de Bordeaux, Jan. 3, 1909.

Hardouin, P., Presse Médicale, Aug. 20, 1910.

Landau, L., Berlin. Klin. Woch., June 15, 1908.

Müller, P., Deutsche Zeit.f. Chir., Aug. 1900.

Romme, R., Presse Médicale, Jan. 23, 1909.

Schlesinger, Semaine Médicale, Mar. 1, 1911.

Smith, H. B., Boston Med. and Surg. Jour., Oct. 14, 1909.

Thoma, F., Deut. Med. Woch., March 19, 1908.

Thomson, Campbell, Acute Dil. of the Stomach, 1902.

Wiesinger, Deutsche Med. Woch., Feb. 1901.

\section{Regurgitant Vomiting}

Braun, Archiv f. Klin. Chir., 1893, xlv. p. 361.

Chlumskij, Beit. z. Klin. Chir., 1898, xx. p. 252.

Mayo, W. J. Annals of Surgery, 1906, xliii. p. 537.

Mauclaire, P., Archiv génér. de Chir., June 6, 1912.

Paterson, H. J., Gastric Surgery, 1906, p. 5.

Terrier, Chirurgie de l'estomac, p. 164.

\section{Diarrhata}

Anchütz, Mittheil. a. d. Grenzgebeit, 1905, xv. p. 305.

Carle and Fantino, Archiv. f. Klin. Chir., 1898, lvi. p. 217.

Kelling, Archiv f. Klin. Chir., 1900, lxii.

\section{Internal Hernia}

Barker, A. E., The Lancet, 1904, ii. p. 1277.

Gordon, T. E., The Lancet, 1905, ii. p. 1477.

Gray, H., The Lancet, 1904, ii. p. 526.

Mayo, W. J., Annals of Surgery, Aug. 1902.

Moynihan, B. G. A., The Lancet, 1906, i. p. 1345. 


\section{CHAPTER VI}

\section{THE PHYSIOLOGICAL EFFECTS OF THE OPERATION OF GASTRO-JEJUNOSTOMY}

WHEN, as was the case a few years back, the operation of gastro-jejunostomy was performed only as a last resort in cases of pyloric stenosis, physiological questions were a secondary consideration. At the present time, when the operation is advised for conditions such as intractable gastric ulcer, in which it is an operation of expediency rather than of necessity, a knowledge of its precise effects on the gastric and metabolic functions of the body must be one of the determining factors in the decision for, or against, operation.

The Presence of Bile in the Stomach.-After the operation of gastro-jejunostomy a certain amount of bile finds its way into the stomach. The quantity varies, and usually diminishes after the first few weeks.

In my own experience, it is rare to find bile in the fasting stomach, and I regard its presence under such conditions as evidence of inefficiency of the efferent opening. The fluid drawn off one hour after a test-breakfast often appears to contain bile, but Gmelin's test usually gives a negative result. If the gastric contents be tested two hours after a test-meal, Gmelin's test is obtainable frequently. I use the expression "obtainable" advisedly, for considerable care is requisite to demonstrate the presence of bile, although from the appearance of the gastric contents one would judge it to be present in some quantity.

Indeed, I believe that the statements as to the amount 
of bile which regurgitates into the stomach are exaggerated. It is probable that the regurgitated bile forms not more than 5 or 6 per cent. of the stomach contents. I need hardly point out that if bile gain entrance into the stomach pancreatic secretion must do so as well.

Granted, then, that there is a permanent reflux of bile into the stomach after gastro-jejunostomy, the question arises, Is its presence harmful? All the evidence indicates that it is not, provided the efferent opening of the anastomosis is efficient. Dastre and Massé have shown that in dogs the presence of bile in the stomach does not interfere with the digestion and general health. The well-known case reported by Sir Berkeley Moynihan, as well as the results of the operation of cholecysto-gastrostomy in man, confirm these observations, while the metabolism experiments, to which I will refer later, afford further evidence to the same effect. Indeed, I think we may affirm safely that, in part at least, the beneficial effects of gastro-jejunostomy on gastric ulcer are due to the entrance of the alkaline pancreatic juice and bile into the stomach.

The Effect of Gastro-jejunostomy on the Gastric Secretion.-The most marked effect of gastro-jejunostomy is the diminution of the total acidity of the gastric contents which follows operation. At first sight it would appear that this diminution is but the result of partial neutralisation of the gastric contents by the bile and pancreatic juice which gain entrance into the stomach. But the problem is not such a simple one.

Space will not permit me to describe in detail the observations which I have made on this subject, so I will indicate briefly what appear to me to be the chief factors at work.

From an examination of a considerable number of cases I find that the average diminution of the total acidity after gastro-jejunostomy is about 30 per cent. There are several 
reasons which appear to me to indicate that this diminution is not due solely to partial neutralisation.

First, judging by the amount of bile usually found in the stomach, the diminution is too great to be the result of a purely chemical reaction.

Secondly, if the diminution were the result of a chemical reaction we should expect all the free hydrochloric acid to be neutralised, but this is not invariably the case. In several instances I have found free hydrochloric acid present, and in those cases in which it has been present before operation usually it can be demonstrated after operation, by giving a suitable test-meal, such as Leube's.

Thirdly, there is a diminution of the total chlorides in the gastric contents which must be the result, not of neutralisation, but of diminished secretion. Two factors, at least, therefore, appear to be at work in bringing about the diminution of total acidity which is found after gastrojejunostomy : (1) a diminution of the total chlorides secreted ; (2) partial neutralisation of the gastric contents by bile and pancreatic juice. The brilliant researches of Pawlow and his co-workers have demonstrated the striking interdependence of the secretions of the digestive glands, and it is this interdependence which seems to me to offer a reasonable explanation of the diminution of chlorides. We may, I think, assume safely, that during the churning movements of the stomach some of the gastric juice will be squeezed, as it were, through the anastomotic opening. This escape of some of the acid will prevent the total acidity rising as high as would otherwise be the case. But it will have a further effect. The presence of an acid fluid in the jejunum causes a secretion of pancreatic juice (Wertheimer).

Thus after gastro-jejunostomy, if my hypothesis be correct, the early presence of acid in the jejunum will lead to a flow of pancreatic juice at an earlier period of digestion 
than in a normal individual. With this earlier stimulation of the pancreas there will be a correspondingly earlier diminution of the gastric secretion, and, as a consequence of this, a diminution in the total chlorides secreted by the gastric mucous membrane. Possibly other factors are at work, such as an inhibitory effect of secretin or other constituent of the pancreatic juice. In those cases in which there has been hyper-acidity before operation, the removal of the abnormal excitation of the gastric mucosa, which results from spasmodic stenosis of the pylorus, will lead to a considerable diminution of the total chlorides, and it is certainly significant, that it is just in these cases of gastric ulcer with hyper-acidity, that the diminution of the total acidity of the gastric contents is most marked.

The Effect of Gastro-jejunostomy on Gastric Digestion.Some authorities maintain that gastric digestion is lost after gastro-jejunostomy. Even if this were true, it would not be of such importance as would appear at first sight, for we know from the experiments of Pachon and Carvalho on dogs, from the observations made on Schlatter's celebrated case of total gastrectomy, as well as from the clinical evidence afforded by those patients who have survived complete removal of the stomach, that Nature is able to dispense with this organ. There is, perhaps, nothing more wonderful in the whole range of surgery than that human beings should not only survive the operation of total gastrectomy, but should remain for years in the enjoyment of perfect health and activity.

Notwithstanding Nature's adaptability, the retention of gastric digestion adds undoubtedly to the resisting power of the individual in the struggle for existence. Personally, I do not believe that loss of gastric digestion is a necessary consequence of a short circuit between the stomach and jejunum. Those who have expressed an opposite view 
base their conclusions on the subsequent diminution of chlorides found in the gastric contents. This argument is not conclusive, as the chlorides may be diminished greatly without the loss of gastric digestion. Further, unless there be advanced atrophy of the mucous membrane owing to disease existing before operation, in my experience there is usually a progressive increase in the chlorides secreted during the digestion of a test-meal. A more conclusive indication of the extent of gastric digestion can be gained from an examination of the fæces. Schmidt has shown that only the gastric juice can digest raw connective tissue. On many occasions after gastro-jejunostomy, I have fed patients on raw beef, but have never found any undigested connective tissue on microscopical examination of the fæces.

The Effect of Gastro-jejunostomy on the Motility of the Stomach.-Impairment of the motility of the stomach is a much more serious matter than impairment of its secretion, for it appears that the stomach, if its peptic power be reduced, endeavours to make up for the failure of digestive power by greater energy in moving on its contents. Under these circumstances the food leaves the stomach incompletely digested, but this does not seem of serious import, for Von Noorden maintains that the intestine can assume vicariously the peptic functions of the stomach. I have pointed out already, that after gastro-jejunostomy some diminution of the gastric secretion occurs; if it were the case, that in addition, gastro-jejunostomy impairs the motility of the stomach, then the usefulness of the operation would be curtailed considerably.

Diverse views have been expressed on this subject. Hartmann and Soupault, Rydygier and Rosenheim, found the motility of the stomach permanently impaired; Carle and Fantino, on the other hand, maintain that the emptying of the stomach is hastened rather than retarded. I think 
it is possible that in such investigations the existence of impaired motility before operation has not always been excluded. Although motor insufficiency be present after operation, we cannot assume that the insufficiency is due to operation, unless we have shown the stomach to have been normal in this respect before operation.

From numerous observations I think we may conclude that, in those cases in which the motility is impaired markedly by pyloric stenosis, or by adhesions, the operation of gastro-jejunostomy results usually in a marked improvement in the evacuation of the stomach contents. In those cases, on the other hand, in which before operation the motility is unimpaired, gastro-jejunostomy may either slightly retard, or slightly hasten evacuation, but inasmuch as this retardation or acceleration falls within the physiological limits, we are justified in saying that in cases in which the motility is normal before operation, the evacuation of the stomach, for practical purposes, is unchanged by gastrojejunostomy.

Is Gastro-jejunostomy a Drainage Operation?-For several years I have been urging the view that gastrojejunostomy is not a drainage operation. The action of gastro-jejunostomy is physiological, not merely mechanical. The correctness of the view I put forward is being confirmed now by the X-ray investigation of cases in which gastro-jejunostomy has been performed. Almost all writers on the subject have attributed the beneficial effects of gastro-jejunostomy on gastric ulcer, to hastened evacuation of the stomach. If this be true it follows, that in those cases of gastric ulcer in which the motility of the stomach is normal, the operation of gastro-jejunostomy should be of no avail. This has not been my experience. Indeed, some of the most striking successes I have witnessed have been cases in which, before operation, there was no evidence of delayed 
evacuation of the stomach. If gastro-jejunostomy act by drainage, gastric lavage would be a simple and equally efficacious substitute.

I think, therefore, that we must look for some other factor than drainage to explain the influence of gastrojejunostomy on gastric ulcer. There is general agreement that hyper-acidity is the factor which prevents the healing of an ulcer. After gastro-jejunostomy there is a marked diminution of the total acidity of the gastric contents, which diminution does not depend on improved evacuation. It is to this lowering of the gastric acidity that the beneficial effects of gastro-jejunostomy are due.

The Effect of Gastro-jejunostomy on the Metabolism of the Human Body.-In the year 1897 Jöslin published some observations on the metabolism of two patients on whom gastro-jejunostomy had been performed. He maintained that, as a result of this operation, there is a marked diminution of the proportion of nitrogen absorbed from the food, and an even more marked diminution in the fat absorption. These observations were accepted without question, and evil consequences were attributed to gastro-jejunostomy of which it is not guilty.

In my experience the average diminution of nitrogen absorption after gastro-jejunostomy is 1.7 per cent. and the average diminution of fat absorption 1.9 per cent. In all the cases I have investigated, the variation of absorption, both of nitrogen and of fat, has been within the limits found in individuals who are in good health. My observations have been repeated by Dr. H. C. Cameron, who has obtained very similar results.

These experimental observations are supported by the evidence of clinical experience. After gastro-jejunostomy patients may live for years in the enjoyment of perfect health. Even more striking are the results of gastro-jejunos- 
tomy for infantile pyloric stenosis. There are children alive and in robust health on whom this operation was performed in early infancy. It is inconceivable that they would have lived for seven or eight years, and developed into strong, healthy children, had the operation the harmful effects which have been attributed to it.

\section{SUMMARY}

To sum up, I think we are justified in drawing the following conclusions as to the physiological effects of gastrojejunostomy :

1. A certain amount of bile and pancreatic juice enter the stomach after gastro-jejunostomy, but the amount is small and has no injurious effect.

2. The acidity of the gastric contents is diminished markedly, usually about 30 per cent. This is due partly to a diminution of the total chlorides secreted, and partly to the partial or complete neutralisation of the free hydrochloric acid by the alkaline bile and pancreatic juice, and probably also to earlier stimulation of the pancreatic secretion, and compensatory earlier fall of the gastric secretion. In ulcer near the pylorus the removal of spasmodic stenosis of the pylorus tends likewise to diminish the total acidity.

3. Gastric digestion is impaired, but not lost, after gastrojejunostomy.

4. The motility of the stomach, if normal before operation, for practical purposes is unaffected. Therefore gastrojejunostomy is not a drainage operation. Its beneficial effects on gastric ulcer are due to the diminution of the acidity of the gastric contents.

5. Gastro-jejunostomy has no material effect on the metabolism of the human body, the percentage of nitrogen and fat absorbed being within the limits observed in indi- 
viduals who are apparently healthy. This chemico-pathological evidence is supported by the evidence of clinical experience.

\section{REFERENCES}

The Physiological Effects of the Operation of GastroJEJUNOSTOMY

Bayliss and Starling, Proc. Roy. Soc., 1902, p. 352.

Cameron, H. C., Brit. Med. Jour., 1908, i. p. 140.

Carvalho, Comptes rendus de la Soc. de Biol., 1893, p. 794.

Dastre, Archiv de Physiol., 1890, ii. p. 316.

Hofman, Munich Med. Woch., May 3, 1898.

Jöslin, Berlin. Klin. Woch., 1897, p. 1047.

Massé, Congrès Frangais de Chir., 1898, p. 300.

Moynihan, B. G. A., Brit. Med. Jour., 1901, p. 1136.

Paterson, Herbert J., Gastric Surgery, 1906, p. 23.

Paterson, Herbert J., Jour. Amer. Med. Assoc., Aug. 24, 1907, xlix. p. 657. Pawlow, The Work of the Digestive Glands (trans. by W. H. Thompson). 


\section{CHAPTER VII}

\section{JEJUNAL AND GASTRO-JEJUNAL ULCERS}

THE first case of jejunal ulcer was reported by Braun in 1899. In 1909 I published a short monograph on the subject, with a review of all cases recorded up till that date.

I pointed out that in a considerable proportion of the cases recorded as jejunal ulcer, the ulcer was situated at the anastomosis, and therefore was gastric as much as jejunal. In order that such ulcers should be distinguished clearly from jejunal ulcer proper, I suggested that they should be described as "gastro-jejunal ulcers." This classification has been adopted by most of those who have written since on the subject.

The characters of these two varieties of ulcer differ, and I believe the conditions determining their occurrence are dissimilar. Jejunal ulcers are the result of altered physiological conditions produced by operation; gastro-jejunal ulcers probably are a direct consequence of operation; their origin is due to faults in technique, and their persistence to hyper-acidity.

Frequency.-It is impossible to estimate with accuracy the proportion of cases in which gastro-jejunostomy is followed by jejunal ulcer. Mikulicz had two cases in 160 gastro-jejunostomies. Schostak has reported that in the Krönlein clinic one instance of jejunal ulcer occurred in 92 gastro-jejunostomies. Wickenhauser observed three jejunal ulcers in 115 gastro-jejunostomies, and Rotgans one in 49 . Jejunal ulcer occurred in three of the 348 patients whose 
after history I have been able to trace. Doubtless some cases have not been recorded, and probably more have not been recognised. Even so the total number would be markedly small in comparison with the enormous number of times the operation of gastro-jejunostomy has been performed. Now that attention has been directed to the subject, doubtless more cases would have been recorded were jejunal ulcer at all common. There can be little doubt that with modern technique it is now a less frequent complication. In the present state of our knowledge I think we are justified in estimating the probable risk of jejunal ulcer following gastro-jejunostomy at under two per cent.

\section{Clinical Groups}

Clinically, the cases of jejunal ulcer hitherto recorded are divisible into two groups :

Group I.-Cases in which perforation into the general peritoneal cavity occurs.

Group II.-Cases in which, owing to the formation of limiting adhesions, perforation does not result in the escape of bowel contents into the general peritoneal cavity.

This group includes two subdivisions :

(a) Cases in which the base of the ulcer becomes adherent to the abdominal parietes, so that perforation results in inflammatory exudation into the abdominal wall.

(b) Cases in which the base of the ulcer becomes adherent to and perforates into a hollow viscus, the colon in the cases so far recorded.

Symptoms. - The clinical picture presented by the cases in which perforation into the general peritoneal cavity occurs, differs materially from that in cases in which general peritonitis is prevented by the formation of protective adhesions. 
Symptoms in Cases belonging to Group I.-In almost all the recorded cases belonging to this group, the patients, after gastro-jejunostomy, had lost all gastric symptoms and had been in good health until, suddenly, "like a bolt from the blue," they were seized with severe abdominal pain, heralding a perforative peritonitis, which in the untreated cases, speedily had a fatal ending.

Symptoms in Cases belonging to Group II.-Pain, varying in intensity, and in many of the cases of a severe character, is the prominent symptom of cases in this group. In some instances the pain was not sufficiently severe to prevent the patients from following, more or less, their usual vocations, for some years before they sought surgical relief ; while in others the patients were reduced to an extreme degree of exhaustion. The pain does not appear to have any definite relation to the ingestion of food, although often it is aggravated by it.

An important sign of jejunal or gastro-jejunal ulcer is the existence of an induration or swelling in the abdominal wall. Usually such a swelling is present if an anterior gastrojejunostomy has been performed. This swelling is the result of the adhesion of the ulcerated area to the anterior abdominal wall. Perforation of the base of the ulcer leads to an inflammatory exudation into the abdominal wall, and consequent induration, and in some instances to a very definite mass. Sometimes the skin over the swelling becomes ulcerated and destroyed with the formation of a jejunal fistula. As a rule, the situation of the swelling is in the left rectus muscle about the level of the umbilicus. Thus the presence of a swelling in the left rectus is highly suggestive, if indeed not diagnostic, of jejunal or gastrojejunal ulcer, while the absence of any induration or swelling makes the diagnosis of this condition more difficult and less certain. When a posterior gastro-jejunostomy has been 
performed, as a rule such a swelling cannot be felt. There is usually hyper-acidity of the gastric contents.

Diagnosis.-In the cases in which perforation into the general peritoneal cavity occurs, the signs and symptoms are similar to those of a perforated gastric ulcer. We cannot distinguish between the two conditions, but it appears that after gastro-jejunostomy a perforated jejunal ulcer is a more likely event than a perforated gastric ulcer.

When protective adhesions are present, the diagnosis of jejunal or gastro-jejunal ulcer must often be uncertain. But this much we can say: that when, after an interval of good health, a patient, who has had gastro-jejunostomy performed, begins to suffer constant pain, especially if the pain be accompanied by hyper-acidity or hyper-secretion, we should bear in mind the possibility of the existence of a jejunal or gastro-jejunal ulcer. When, in addition to pain, there is an induration or swelling of the abdominal wall in the neighbourhood of the stomach, the diagnosis is almost certain. We must remember, however, that a swelling or induration rarely is present in patients on whom the posterior operation has been performed. For practical purposes, we may say that every case of recrudescent pain of a constant character after gastro-jejunostomy, should be regarded as a case of potential jejunal or gastro-jejunal ulcer, and treated accordingly.

Pathology.-First, as to jejunal ulcer proper. This in appearance and clinical course bears a close resemblance to the ordinary round ulcer of the stomach and duodenum. As in the stomach, we have the "punched-out" ulcer suggestive of rapid destruction of tissue, apt to perforate before protective adhesions are formed. Naturally, the thin jejunal wall is destroyed more readily by ulceration than is the gastric wall, with its thicker musculature and tough submucous layer. Then, too, we have the jejunal ulcer (Fig. 48) 
with shelving thickened edges, more chronic in course, becoming adherent to neighbouring structures, finally perforating and leading to inflammatory infiltration of the adherent structures. Jejunal ulcers are situated most commonly in the efferent limb, either opposite to or just below the anastomosis, an observation which has a bearing on the ætiology of these ulcers.

Secondly, as to gastro-jejunal ulcer (ulcer at the site of

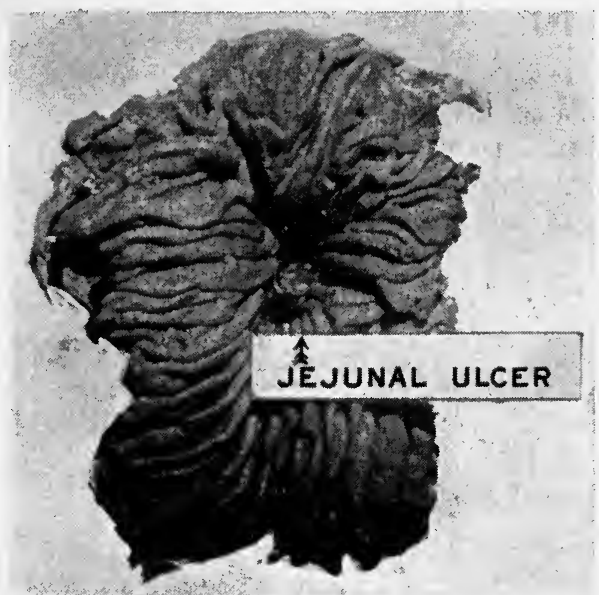

FIG. 43.-A portion of resected jejunum, showing a chronic jejunal ulcer. From a woman aged fifty-two years, on whom an anterior gastro-jejunostomy had been performed five years previously.

anastomosis). Gastro-jejunal ulcer lacks the definite localised appearance of the jejunal ulcer. It appears as an irregular ulceration round the margin of the anastomotic opening, sometimes invading the jejunal as well as the gastric mucosa. If healing occur, the process of cicatrisation may lead to partial, or even complete, obliteration of the communication between the stomach and jejunum. Gastro-jejunal ulcers are less likely to perforate into the general peritoneal cavity than are jejunal ulcers.

Etiology.-Various hypotheses have been adduced as furnishing the explanation of jejunal ulceration following 
gastro-jejunostomy; and although, until our knowledge, as to the causation of gastric and duodenal ulcers, is more precise, conclusions regarding the ætiology of jejunal ulcers may require subsequent modification, nevertheless, we can form fairly reliable indications as to the conditions favouring its occurrence, and so come to some conclusions as to the best means of prevention and treatment. The three chief hypotheses which have been put forward to explain the occurrence of jejunal ulcers are as follows :

1. That jejunal ulceration is due to circulatory disturbances in the attached jejunum.

2. That jejunal ulcer is an infective process.

3. That jejunal ulcer is due to the digestive action of gastric juice on mucous membrane accustomed to the presence of alkaline contents only.

The "peptic" hypothesis has been adopted by most of those who have written on this subject, so that jejunal ulcer following gastro-jejunostomy usually is spoken of as. "peptic" jejunal ulcer. This appears to me to be putting the cart before the horse; the digestion of the living cells. must be the consequence of their injury by the hydrochloric acid of the gastric juice. The peptic action is secondary, the primary factor being the injury of the mucous membrane by the toxic action of the hydrochloric acid. I believe that the amount of free hydrochloric acid, not the total acidity of the gastric contents, is the important factor. The free hydrochloric acid acts as a poison on the cells of the mucous membrane, so injuring them that they fall a ready prey to digestive action. In a normal individual, probably all the free hydrochloric acid of the gastric contents is neutralised before the chyme leaves the duodenum. Now after gastro-jejunostomy, the gastric contents pass directly into the jejunum. Two circumstances, however, prevent the diversion of the gastric contents from exercising an 
injurious effect upon the jejunal mucous membrane. As I have pointed out elsewhere, after gastro-jejunostomy there is, first, a distinct diminution of the chlorides secreted by the stomach, and secondly some regurgitation of bile and pancreatic juice into the stomach. In consequence of these changes the percentage of free hydrochloric acid in the gastric contents is diminished markedly, so that after a satisfactory gastro-jejunostomy the amount of free hydrochloric acid entering the jejunum is so small, that it is neutralised at once by the bile and pancreatic juice.

My view is that jejunal ulcer following gastro-jejunostomy is the result of a toxic agent or poison which so injures or kills the cells of the jejunal mucous membrane that they are readily digested by the intestinal juice. The toxic agent usually present is free hydrochloric acid, but possibly other toxic substances may be present, and either may increase the effect of the other. Thus, a small percentage of free hydrochloric acid in the jejunum, which by itself would not cause ulceration, may in the presence of some other toxic agent, produce ulceration. The circumstances under which free hydrochloric acid may be present in the jejunum are :

1. Hyper-acidity of the gastric contents, so that the bile and pancreatic juice are unable to neutralise completely all the acid entering the jejunum.

2. Normal percentage of hydrochloric acid in the gastric juice but excessive secretion, so that the amount of hydrochloric acid discharged into the jejunum is greater than can be neutralised.

3. Diversion of the course of the bile and pancreatic juice, so that part of the jejunum is exposed to the action of the gastric contents unmixed with bile and pancreatic juice as after operations of the "Y type," and gastro-jejunostomy with entero-anastomosis. 
4. Normal acidity and normal amount of gastric secretion, but incomplete neutralisation in the jejunum, owing to temporary diminution of the flow of bile and of the secretion of pancreatic juice.

In a few cases jejunal ulcers are probably of infective origin. Such cases present the following features: $(a)$ the ulcers occur within a very short period after gastro-jejunostomy; (b) usually the ulcers are multiple.

So far I have discussed very briefly the ætiology of jejunal ulcer proper. Much of what has been said applies also to gastro-jejunal ulcer. Hyper-acidity is an important factor in both, but there is this differerce: jejunal ulcers are a result of altered physiological conditions produced by operation ; probably gastro-jejunal ulcers are a direct consequence of operation. When a wound becomes ulcerated, the presumption is that the ulceration is a consequence of the wound. Beyond the general rule that jejunal ulcers occur most commonly in that part of the jejunum in which the acidity of the intestinal contents is greatest, we do not know what determines the site and area of ulceration. The site of a gastro-jejunal ulcer is determined by the wound made in effecting the anastomosis, and the ulceration persists and spreads if there be hyper-acidity of the gastric juice.

In connection with the origin of gastro-jejunal ulcer, the question arises, Does primary union take place between the mucous membranes of the stomach and jejunum when they are maintained in apposition by the use of an inner suture ? It is obvious that the necrosis produced by a Murphy's button must result in a ring of ulceration which heals by cicatrisation. Non-apposition of the gastric and jejunal mucous membranes, injury to the mucous membrane during the operation, or infection of the suture used for approximation must also result in healing by granulation. Drs. A. H. 
Gould and Harrington, ${ }^{1}$ as the result of experiments on animals, conclude that an inner layer of stitches has little influence upon the healing of the wound. They found that whether the mucous membranes were apposed by suture or not, the mucous membrane round the margin of the anastomosis sloughed, and the separation of the slough left an ulcer which became covered over with mucous membrane in about three weeks. If this be true, then it follows that every gastro-jejunostomy is followed by a gastro-jejunal ulcer. If hyper-acidity or hyper-secretion exist, then the ulcer, instead of healing, may persist or spread, and eventually cause symptoms such as have been described in an earlier part of this chapter.

I must state my belief, that in human beings primary union of the gastric and jejunal mucous membrane may occur. To attain such a result rigid asepsis is imperative, an impossibility in operations on animals. In human beings, on the other hand, by adequate preparatory treatment and by careful dieting, it is possible to render sterile at least the upper part of the gastro-intestinal tract, and so to operate under conditions more favourable for primary union than can be obtained in experimental work on animals. I have had an opportunity of examining a considerable number of anastomoses from patients who have died at various intervals after gastro-jejunostomy. In all I found direct continuity between the gastric and jejunal mucous membrane, and, further, the thread used for suturing was encapsuled and had not been discharged into the intestine. In several instances microscopical preparations from recent anastomoses showed continuity of mucous membrane, and no evidence of

1 The Technique of Operations on the Intestines and Stomach, 1906, p. 20. The process of repair following the use of Murphy's button has been investigated by Barbat, and F. T. Murphy has described the process of healing after anastomosis by means of McGraw's elastic ligature. A. H. Gould, in the work to which I refer, gives an admirable summary of these researches. 
the sloughing which Gould states is the invariable rule. Whether primary union be possible or not, we should employ the means most calculated to obtain it, and of this at least I am convinced-that the use of an inner suture ensures an opening which subsequently contracts less than an anastomosis effected by a button, or by the use of serous sutures only.

The regurgitation of bile and pancreatic juice which takes place into the stomach after simple gastro-jejunostomy, must be favourable to the union of the apposed surfaces, by diminishing the acidity of the gastric contents as they pass through the opening, and Nature in her wisdom has so ordered it, that this regurgitation is at its maximum just when it is most needed-in the early days after operation. This protective regurgitation, so far as I have observed, is absent in operations of the "Y type" and when an anteroanastomosis is performed.

The Closure of Gastro-jejunostomy Openings. - A few years ago we heard a good deal about the closure of gastrojejunostomy openings in cases of patent pylorus. The Radiographers stated that after gastro-jejunostomy the food continues to pass out through the patent pylorus, and it was assumed that as the anastomotic opening becomes functionless, it gradually contracts, or becomes completely closed. Now it is obvious, that closure of a gastro-jejunostomy opening premises ulceration. The closure is the consequence of a gastro-jejunal ulcer.

A gastro-jejunal ulcer is the result of inaccurate apposition of the mucous membrane, or too tight constriction thereof. The ulcer may heal speedily, or may extend and result in marked contraction or obliteration. Now just as hyper-acidity is the condition which prevents healing of a gastric ulcer, so hyper-acidity plays the chief rôle in the prevention of the healing of a gastro-jejunal ulcer. Closure 
of the anastomotic opening occurs, not because the pylorus is patent, but because the cases in which the pylorus is patent usually are cases of ulcer with hyper-acidity. The hyper-acidity is the causal factor, not the patent pylorus. It is in such cases that gentle handling of the mucous membrane, careful application of the sutures, rigid asepsis, and strict dieting after operation are especially important, in order to prevent the occurrence of gastro-jejunal ulcer with its attendant risk of subsequent contraction of the opening.

If jejunal ulcer be the result of the toxic action of hydrochloric acid on the jejunal mucous membrane, it follows that operations of the "Y type" are wrong in principle. After this type of operation the bile and pancreatic juice enter the jejunum some inches below the anastomosis, so that this and several inches of jejunal mucous membrane are exposed to the action of unneutralised gastric contents. Mons. le Dr. Monprofit, one of the few advocates of this operation at the present time, has obtained good results from it; nevertheless, I think that it is a method which, if employed at all, should be used only in those cases in which free hydrochloric acid is absent from the gastric contents. Primary entero-anastomosis is an unnecessary and now seldom performed complication of gastro-jejunostomy, and is open to the same objection as gastro-jejunostomy " En-Y." Inasmuch as the relative frequency with which these methods have been employed is unknown, we cannot prove that they are followed by jejunal or gastro-jejunal ulcer more frequently than simple anterior or posterior gastro-jejunostomy. But if, as is almost certainly the case, operations of the "Y type" (including entero-anastomosis) have been far less commonly performed than the simple anterior or posterior method, it is perhaps not without significance that in 24 per cent. of the recorded cases, jejunal or gastro-jejunal 
ulcer has followed gastro-jejunostomy " $\mathrm{En}-\mathrm{Y}$ ” or gastrojejunostomy with entero-anastomosis.

A perusal of the recorded cases shows that a large proportion have followed the anterior operation, and it has been argued that this method predisposes to jejunal ulceration. At first sight this appears to be the case, but I do not think that a closer examination of the records justifies this assumption.

Very few instances of jejunal ulcer following a posterior, short-loop operation have been recorded. It has been suggested that the upper part of the jejunum is more resistant than the lower to the toxic action of hydrochloric acid. I think this is doubtful. Possibly the comparative immunity of the short-loop operation may be due, not to any difference in the physiology of this method, but to improvements in technique in gastric operations in general.

The Cause of Hyper-acidity after Gastro-jejunostomy.I have already pointed out that after gastro-jejunostomy there is a marked fall in the total acidity of the gastric contents, the results of the altered physiological conditions effected by the operation. This diminution, as a rule, is sufficient to convert hyper-acidity before operation into hypo-acidity after operation. What, then, are the causes of hyper-acidity after operation? A clear distinction must be drawn between two classes of case :

1. Cases in which hyper-acidity is present before and persists after operation.

2. Cases in which, after a period of normal or subnormal acidity following operation, the gastric contents subsequently become hyper-acid.

Hyper-acidity may persist after operation for the reason that before operation the hyper-acidity was so extreme, that the diminution caused by gastro-jejunostomy is insufficient to reduce the acidity of the gastric contents to normal, 
unless the patient be kept on a milk diet for some months. Hyper-acidity may persist also because of a too small or inefficient or defective anastomotic opening.

Hyper-acidity of the gastric contents succeeding a period of normal or subnormal acidity is due probably to one of two causes; too small an opening, or indiscretions in diet.

In some cases both these factors may be present. When gastro-jejunostomy is performed on a dilated stomach, the operation usually is followed by a considerable diminution of the size of this organ, and so it may happen that an opening originally of good size may become too small owing to the contraction of the stomach around it. Hayem states that the presence of an acid liquid in a fasting stomach is an indication that the opening does not allow of complete evacuation of the stomach. A small or inefficient opening leads to gastric stasis, gastritis, and hyper-acidity. I quite admit that a slight degree of gastric stasis may have no ill result; but it is reasonable to argue that in such a case a slight attack of gastritis due to some error in diet, insufficient to cause trouble if the anastomotic opening were larger, may lead to such a degree of gastric stasis that hyper-acidity ensues. Doubtless in some cases long-continued indiscretions in diet may lead to gastritis, however large the opening may be.

Treatment.-The treatment of the cases in which perforation into the general peritoneal cavity occurs is obvious. Immediate laparotomy and suture of the perforation offer the only prospect of saving the life of the patient. If the patient recover, the condition of the gastric contents should be investigated, and if hyper-acidity, hyper-secretion, or gastric stasis exist, the gastro-jejunostomy opening should be enlarged, or a new gastro-jejunostomy performed.

In the cases of Group II in which protective adhesions are present, the indication for surgical treatment is not so 
clear. The results of operation are not such as to encourage us to resort to surgery until after a trial of medical treatment.

In connection with medical treatment the question arises, What evidence have we of the spontaneous healing of jejunal and gastro-jejunal ulcers? In some of the recorded cases obliteration of the gastro-jejunostomy opening has occurred, and this obliteration must have been the result of cicatrisation following ulceration. It is true that in those cases surgical intervention was necessary subsequently, nevertheless they show that ulceration at the site of the anastomosis under some circumstances may heal spontaneously.

The evidence as to the possibility of permanent relief by medical treatment is very inconclusive. All we can say for certain is, that occasionally jejunal and gastro-jejunal ulcers heal, but the process of healing is apt to result in a condition requiring surgical intervention.

Medical Treatment.-In my judgment, before an operation is performed, the patient should undergo a course of careful dietetic and medicinal treatment for two reasons: first, there is just a possibility, remote it is true, that in this way a cure may be brought about; and secondly, if subsequently operation be necessary, success will be more likely to follow if, as the result of medical treatment, the acidity of the gastric contents be diminished. The patient should be kept at absolute rest in bed, and on a diet of milk and eggs, as recommended by Lenhartz for the treatment of gastric ulcer. Bismuth, combined with small doses of hydrocyanic acid, usually relieves the pain, and the researches of Pawlow appear to me to show conclusively the value of the administration of alkalis in diminishing the acidity of gastric contents, an opinion confirmed by my experience in the cases I have seen.

If under medical treatment the pain disappear and the 
acidity become diminished, we may persevere in the hope of ultimate cure, and the treatment should be prolonged for six months at least. The two indications for surgical treatment appear to me to be : first, persistence of the pain and hyper-acidity in spite of treatment, and secondly, evidence of hyper-secretion, or of gastric stasis. The presence of these conditions indicates that the anastomotic opening is at fault, and under these circumstances there is little likelihood of complete and permanent cure without surgical intervention.

Surgical Treatment.-The surgical treatment adopted must depend necessarily on the condition found at operation. Adhesions must be separated, and the perforation sutured, if there be one. A study of the recorded cases indicates plainly, that the less extensive the operation performed, the better is the result, especially if marked hyperacidity exist. The anastomotic opening should be examined carefully, and if it be small it should be enlarged, or a new gastro-jejunostomy performed. In my judgment the former is the preferable course, as it is not only simpler, but does not necessitate a "Y type" operation, which, for reasons already given, is not to be recommended. If, on account of severe pain or rapid loss of strength, operation has to be undertaken before an attempt has been made to reduce any hyper-acidity which is present, I would suggest that the enlargement of the gastro-jejunostomy should be deferred; but if, on the other hand, a strict course of medical treatment has been ineffectual in reducing acidity, then no object is gained by deferring the completion of whatever operative procedure is requisite.

In a number of cases, unfortunately too numerous, the symptoms have recurred after surgical treatment. In such it might be worth trying the effect of a gastro-jejunostomy or " En-Y," but with implantation of the proximal limb of 
the jejunum into the stomach, so that the bile and pancreatic juice are diverted directly into the stomach.

Our great aim should be the prevention of jejunal or gastro-jejunal ulcer, and I will indicate very briefly, under two headings, what appear to me the points of importance in this connection :

(1) The technique of gastro-jejunostomy.

(2) The after-treatment.

(1) The Technique of Gastro-jejunostomy

First, I would emphasize the necessity for a large opening. There is invariably some contraction of the opening, and in cases of dilated stomach subsequent diminution in the size of this viscus still further narrows the communication between the jejunum and stomach.

Secondly, accurate apposition of the mucous membrane of the stomach and jejunum, so as to secure, if not primary union, at any rate, union with the formation of a minimum of scar tissue.

Thirdly, careful application of the inner suture so as to control bleeding, but, at the same time, so as to avoid such a degree of tightness as will cause localised necrosis of the tissues.

Fourthly, the use of simple suturing in preference to mechanical appliances, especially when hyper-acidity is present.

Fifthly, the avoidance of entero-anastomosis and of " $\mathrm{Y}$ type " operations.

It has been suggested that catgut should be used for the inner suture in preference to silk or linen, on the ground that the latter is more apt to serve as a septic focus. I do not think there is a particle of evidence in favour of this assumption. 


\section{(2) The After-treatment of Gastro-jejunostomy}

The necessity for prolonged after-treatment in cases of gastro-jejunostomy, perhaps has not received the attention which it deserves. My rule is to advise all patients whose gastric contents have been hyper-acid before gastro-jejunostomy, to avoid meat in any form for six months at least, and until such time as examination shows that the gastric acidity is sub-normal. The immediate relief which is experienced by patients on whom gastro-jejunostomy has been performed, tempts them to indulge in food unsuited to the condition of the gastric mucosa. In most cases in which gastro-jejunostomy is necessary, the mucous membrane is chronically inflamed, and many months must elapse before it is restored to a healthy condition.

\section{SUMMARY OF CONCLUSIONS}

1. The risk of jejunal ulcer following gastro-jejunostomy is probably under two per cent.

2. At the present time apparently this complication occurs less frequently than formerly.

3. Clinically, there are two groups of cases : (1) those in which perforation into the general peritoneal cavity ensues; (2) those in which general peritonitis is prevented by the formation of adhesions.

4. Pathologically the cases may be classified as follows: (1) ulcers of the jejunum ; (2) gastro-jejunal ulcers, or ulcers at the site of the anastomosis.

5. In some instances jejunal ulcers are of infective origin ; in these cases ulceration commences within a very short interval after gastro-jejunostomy, and usually the ulcers are multiple.

6. In a large proportion of cases the ulcer is single, and is probably the result of the toxic action of hydrochloric acid, 
which injures the cells of the mucous membrane so that they are digested by the intestinal juice. Possibly, other agents than hydrochloric acid may play a part in injuring the mucous membrane.

7. Gastro-jejunal ulcers are a direct consequence of the wound made in effecting the anastomosis, and their persistence is probably the result of hyper-acidity of the gastric juice.

8. Closure of a gastro-jejunostomy opening is the consequence of cicatrisation of a gastro-jejunal ulcer. It is more likely to occur when the pylorus is patent, not because of the patency of the pylorus, but because in such cases, hyperacidity usually is marked.

9. Any procedure or disease which diminishes the amount of bile and pancreatic juice in the jejunum favours the occurrence of jejunal and gastro-jejunal ulcer. For this reason operations of the "Y type" and entero-anastomosis are inadvisable, at any rate in cases in which free hydrochloric acid is present in the gastric contents, as after these procedures the anastomosis and a portion of the jejunum are deprived of the protective influence of the alkaline bile and pancreatic juice.

10. The reason that ulceration has followed the anterior operation more frequently than the posterior operation with a loop, is probably that in former times the anterior operation was more frequently performed.

11. As so few instances of ulcer after the posterior shortloop operation have been recorded as yet, for the present we must assume that its occurrence after this type of operation is less likely. It is possible, however, that this immunity is partly the result of improvements in technique and in the after-treatment of gastric operations in general.

12. In cases in which perforation into the general peritoneal cavity occurs, immediate laparotomy offers the only chance of saving the patient's life. 
13. Inasmuch as there is some evidence that jejunal and gastro-jejunal ulcers may heal, an operation should not be performed in the chronic cases, until after a thorough trial of medical treatment.

14. Even when surgical intervention is necessary an attempt should be made first to diminish hyper-acidity, if this be present.

15. Our aim should be to prevent the occurrence of this complication of gastro-jejunostomy. Preventive treatment consists in (1) careful and appropriate surgical technique, and (2) prolonged after-treatment.

16. Lastly, every case of recrudescent pain of a constant character after gastro-jejunostomy should be regarded as a case of potential ulcer, and treated accordingly, especially when the pain is associated with hyper-acidity or hypersecretion.

\section{BIBLIOGRAPHY}

Battle, Lancet, 1906, i. pp. 274, 1246.

Braun, Verhandl. d. deutsch. Gesellsch. f. Chir., 1899, ii. p. 94.

Brodnitz, Verhandl. d. deutsch. Gesellsch. f. Chir., 1903, i. p. 77.

Cackovic, Liecnicki viestnik, 1903, No. 7.

Connell, Gregory, Surg., Gynoec., and Obstet., Chicago, 1908, vi. p. 39.

Czerny, Beitr. f. klin. Chir., 1903, xxxix. p. 98 (supplement).

Delaloye, Deutsch. Zeitschr. f. Chir., 1906, lxxxiii. p. 518.

Edington, Glasg. Med. Journ., 1907, lxvii. p. 463.

Fraenkel, Deutsch. Arch.f. klin. Med., Leipz., 1905, lxxxiv. p. 217.

Goepel, Verhandl. d. deutsch. Gesellsch. f. Chir., 1902, i. p. 108.

Gosset, Rév. de Chir., Par., 1906, xxxiii. p. 59.

Graser, Verhandl. d. deutsch. Gesellsch. f. Chir., 1906, i. p. 95.

Hadra, Verhandl. d. deutsch. Gesellsch. f. Chir., 1900, i. p. 152.

Hahn, Verhandl. d. deutsch. Gesellsch. f. Chir., 1899, i. p. 74.

Hamann, Cleveland Med. Journ., 1907, vi. p. 183.

Heidenhain, Verhandl. d. deutsch. Gesellsch. f. Chir., 1902, i. p. 108.

Herczel, Congrès de la Soc. internat. de Chir., Brussels, 1905, p. 139.

Hofmann, Beitr. z. klin. Chir., 1906, 1. p. 736.

Jahr, Berl. klin. Wochenschr., 1905, xlii. (Festnummer Ewald) p. 100.

Kaufmann, Mitteil. a. d. Grenzgebeit. d. Med. u. Chir., 1905, xv. p. 151.

Kelling, Verhandl. d. deutsch. Gesellsch. f. Chir., 1906, i. p. 78. 
Key, Einar, "Bidrag till den kirurgiska Behändlingen af Ulcus Ventriculi," Stockholm, 1907.

Kocher, Verhandl. d. deutsch. Gesellsch. f. Chir., 1902, i. p. 103.

Körte, Verhandl. d. deutsch. Gesellsch. f. Chir., 1900, i. p. 137.

Kreuzer, Beitr. z. klin. Chir., 1906, xlix. p. 460.

Lyle, New York Med. Journ., 1906, lxxxiv. p. 1230.

Maylard, The Lancet, 1910 , i. p. 497.

Mayo, W. J., Surgery, Gynoecology, and Obstetrics, Mar. 1910, p. 227.

Mikulicz, Verhandl. d. deutsch. Gesellsch. f. Chir., 1899, i. p. 74, and Boston Med. and Surg. Journ., 1903, cxlviii. p. 609.

Moynihan, Universal Medical Record, Jan. 1912. i. p. 11.

Neumann, Deutsch. Zeitschr. f. Chir., lviii. p. 270.

Nyrop, quoted by Einar Key, Ugeskr. f. Laege, 1907, p. 54.

Paterson, Gastric Surgery, 1906, p. 20, and Jejunal and Gastro-jejunal Ulcer, 1910.

Quénu, Bull. et Mém. de la Soc. de Chir. de Paris, 1902, xxviii. p. 250 ; and also 1904, xxx. p. 194.

Robson, Mayo, Med.-Chir. Trans., Lond., 1904, lxxxvii. p. 339, and Brit. Med. Jour., 1912, i. p. 1.

Rotgans, Congrès de la Soc. internat. de Chir., Brussels, 1905, p. 170.

Schloffer, Wien. klin. Wochenschr., 1903, xvi. p. 492, and Beitr. z. klin. Chir., 1902, xxxii. p. 357.

Schostak, Beitr. z. klin. Chir., 1907, lvi. p. 360.

Steinthal, Verhandl. d. deutsch. Gesellsch.f. Chir., 1900, i. p. 139.

Tiegel, Mitteil. a. d. Grenzgebeit. d. Med. u. Chir., 1904, xiii. p. 897. 


\section{CHAPTER VIII}

\section{GASTRIC ULCER, ITS PATHOLOGY, SYMPTOMS, AND DIAGNOSIS}

Clinically, there are two varieties of gastric ulcer, the acute and the chronic. Whether these varieties are distinct pathologically is doubtful. Probably they are not. It is reasonable to suppose that the chronic ulcer originates as an acute ulcer, which, instead of healing, persists in the form we recognise as a chronic ulcer.

Pathologically the following varieties of gastric ulcer are described :

\section{(i) Erosions}

(a) Simple erosions, which appear as minute superficial abrasions of the gastric epithelium. During life these erosions are seen as numerous bleeding points, but after death they are difficult to detect.

(b) Exulceratio simplex of Dieulafoy.-This is simply a more extensive erosion with greater destruction of the surface epithelium. In some cases the blood-vessels of the muscularis mucosæ are exposed, and if ulcerated, very severe hæmorrhage may ensue.

\section{(ii) Simple Ulcer}

(a) The acute round ulcer, much written of but seldom seen. Its edges are sharply defined and clear-cut, giving it a punched-out appearance. As a rule, more of the gastric mucosa is destroyed than of the deeper layers, so that the ulcer has the shape of a funnel or truncated cone with its 
base at the mucous surface and its apex towards the peritoneal surface of the stomach. The edges of the ulcer are not indurated, but there is often considerable œedematous swelling of the surrounding gastric wall.

(b) Chronic Ulcer.-The commonest variety of gastric ulcer, and the one which requires surgical treatment most frequently. Chronic ulcers vary greatly in size and shape. Usually they are oval or irregular rather than round, the edges being much indurated and raised above the level of the surrounding mucosa. The mucous membrane round the ulcer is deeply congested, numerous varicose veins often being visible. Usually the floor of the ulcer is composed of the muscular wall of the stomach, which is exposed by the destruction of the mucous membrane. In other instances, the whole of the gastric wall may be destroyed, so that the floor of the ulcer is formed by some neighbouring organ, such as the pancreas, to which the stomach, at the seat of ulceration, has become adherent before complete destruction of the whole thickness of the gastric wall has ensued.

Formerly, it was believed that gastric ulcer occurs far more frequently in women than in men. Since surgical activity has invaded the field of gastric disease, our ideas as to the sex-incidence of ulcer have been modified considerably.

We know now that symptoms, supposed to be pathognomonic of gastric ulcer, may arise from other abdominal diseases, such as chronic appendicitis, or gall-stones.

Formerly it was taught that sixty per cent. of gastric and duodenal ulcers occurred in women. According to Drs. W. J. and C. H. Mayo sixty-two men are affected to thirty-two women.

As, however, duodenal ulcer is much more frequent in the male, the incidence of true gastric ulcer in the two sexes is nearly equal, fifty-two men to forty-eight women. 
Plate XXI.

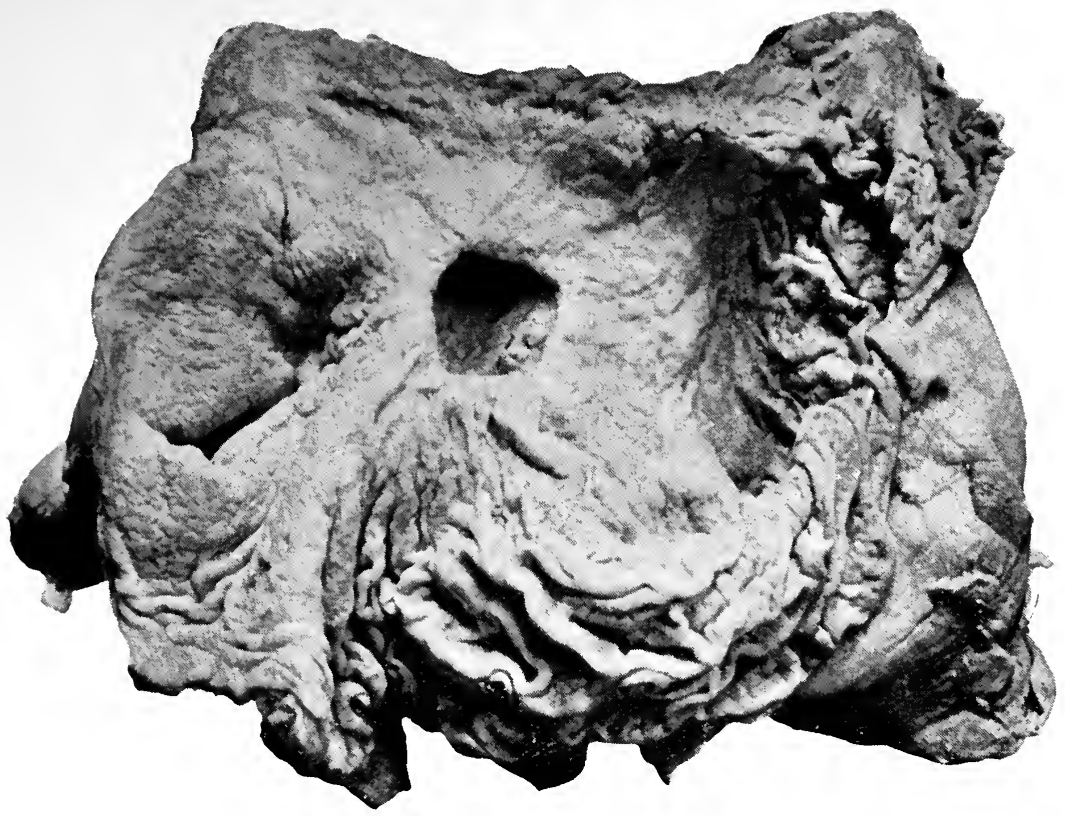

FIG. 49,-Chronic ulcer of quadrilateral shape on the posterior wall of the stomach, which formed a deep excavation on the substance of the pancreas. (W. Soltau Fenwick.)

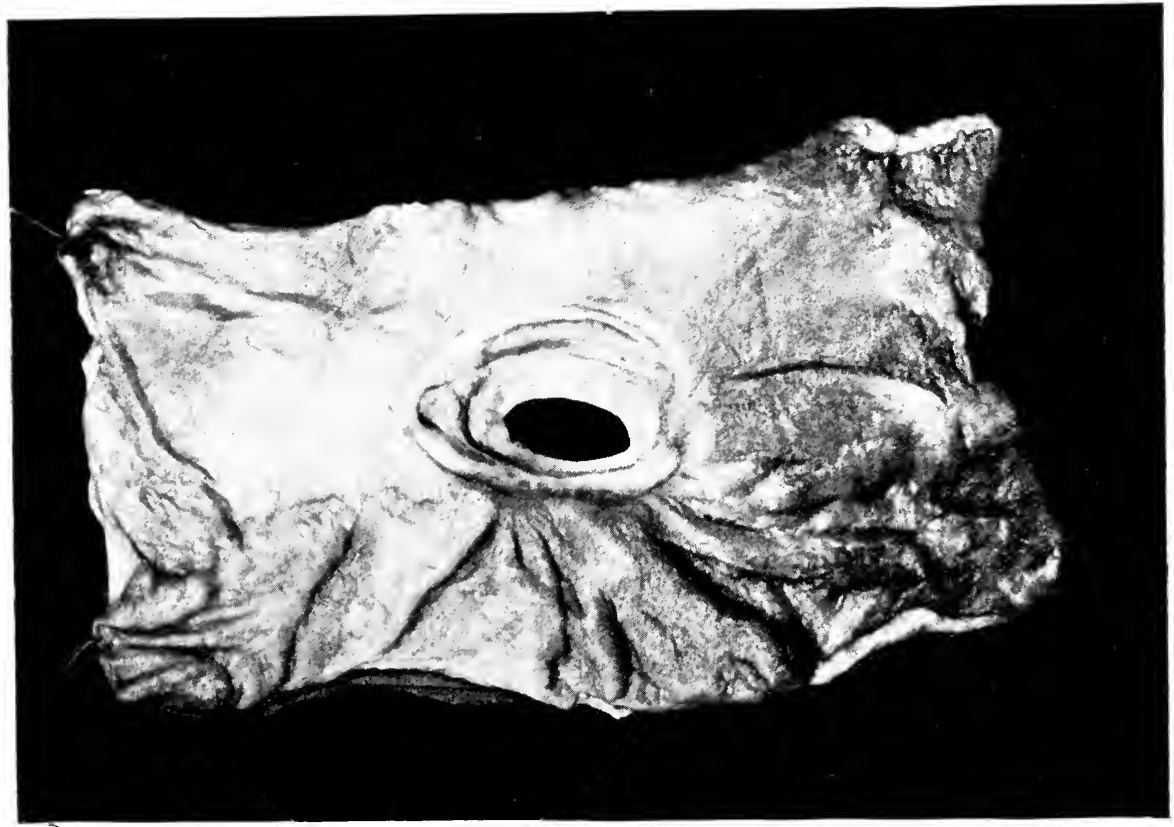

FIG. 50.-A chronic ulcer. The "terraced" appearance of the ulcer is well shown. (St. Bartholomew's Hospital MIuseum, No. 1911.) 


\section{SYMPTOMS}

The three cardinal symptoms of gastric ulcer are: pain, hæmorrhage, and vomiting.

Pain.-This is by far the most important, most constant, and most characteristic symptom. It varies from slight discomfort, sometimes a little more than a feeling of fullness, to pain of such intensity that the patient is " doubled up."

Usually, it is referred to the epigastrium, and is limited to an area of from two to three inches in extent. Sometimes, in cases of ulcer towards the cardiac end of the stomach, the patient is very definite in localising the pain to the left of the middle line. As a general rule, the nearer the ulcer to the pyloric end, the more often is the pain referred to the right of the middle line; but the site of the pain is not an infallible guide to the situation of the ulcer.

Radiation of the pain to the back is not so common as text-books would have us believe, but sometimes the pain radiates to the shoulders.

The pain comes on from half an hour to one hour after food: the nearer the ulcer is situated to the pyloric end of the stomach, the longer the advent of pain is delayed. In gastric ulcers close to the pylorus, the pain may not come on for three or four hours, and in such cases the differentiation from duodenal ulcers is difficult. (See illustrative case, p. 122.)

The pain increases in intensity as digestion progresses, and is at its maximum before the time of the next meal, so to the patient it seems as if the pain were the result of want of food. Sometimes taking more food relieves the pain, but this is not the case so often as in duodenal ulcer. Relief is obtained also by vomiting, whether spontaneous or artificially induced. 
It is common for those suffering from gastric or duodenal ulcer to state that the pain wakes them up in the early morning hours, and they find by experience that it is advisable to keep a glass of milk by the bedside, so that on waking they can drink it, and so secure temporary relief.

Almost all sufferers from gastric ulcer are troubled with flatulent distension of the stomach; the gas is belched up, often with small quantities of fluid, which is very bitter or acid to the taste (water-brash).

Hæmorrhage.-Hæmorrhage from gastric ulcer is not so frequent as is supposed. Hæmatemesis is not due necessarily to gastric ulcer, and so it is not certain evidence of ulcer, neither does its absence contra-indicate a diagnosis of gastric ulcer. The amount lost may be so small that repeated examinations of the fæces are necessary to detect its presence (occult blood), or may be so great that it proves fatal immediately.

If vomited soon after the hæmorrhage has occurred, the blood is bright red in colour, and clots may be present. If the blood has been retained in the stomach for any length of time, the vomited material may be dark red, or a dirty brown colour (the so-called "coffee ground" vomit). If blood in considerable quantity pass through the pylorus, it gives rise to black stools (melæna).

Vomiting.-In the early stage of gastric ulcer, vomiting is infrequent. It may occur if the patient over-indulge in food, but as a rule a patient with a gastric ulcer learns by experience to avoid this indiscretion. When it occurs, vomiting comes on usually some hours after food, when the pain is at its worst.

Some patients produce vomiting artificially, in order to empty the stomach and so gain relief from the pain. Some years ago I operated on a lady who, in order to conceal the severity of her symptoms from her husband, took an ordinary 


\section{SURGERY OF THE STOMACH}

dinner every night and immediately afterwards made herself vomit, to prevent the onset of the pain which she knew would follow invariably within an hour or so.

Vomiting in gastric ulcer may be due to one of three causes :

(i) In the early stages, vomiting of partially digested food, without much mucus, is reflex-Nature's effort to get rid of the irritating food.

(ii) In the later stages vomiting of small quantities of ill-digested food, with much mucus, is the result of chronic gastritis.

(iii) Vomiting of large quantities of sour fluid with partially digested food is an indication of pyloric stenosis, or hour-glass stomach.

Physical Examination.-As a rule there is tenderness over the epigastrium, but this is not constant. Between the attacks physical examination may reveal nothing abnormal. Sometimes the induration and adhesions round the ulcer form a mass sufficiently large to be felt on palpation. This is very important, as the presence of a swelling may lead to a diagnosis of cancer. In many such instances, patients have been denied operation which would have given them complete relief.

Examination of the Gastric Contents.-Hyper-secretion is not an invariable accompaniment of gastric ulcer. In more than half the cases the stomach is empty in the early morning. In 40 per cent. of the cases from 20 to 50 c.c. of clear acid fluid may be withdrawn by aspiration in the early morning. Unless the ulcer be near to the pylorus, or cicatrisation has resulted in pyloric stenosis or an "hourglass" constriction, the motility of the stomach is unimpaired; food-remains are not present in the morning, and the amount recovered after a test-meal is not greater than in health. When there is pyloric stenosis, food-remains 
are present in the morning, and the amount of gastric contents recovered on withdrawing the test-meal may be very considerable.

When a gastric ulcer is situated near to the pylorus, as a rule, the free hydrochloric acid is increased; on the other hand, when the ulcer is in the middle or at the cardiac end of the stomach, the free hydrochloric acid is diminished. In cases of hour-glass stomach free hydrochloric acid is absent.

Probably the free hydrochloric acid always is increased in the early stage of the disease. Later, there is a progressive diminution, so that when marked pyloric stenosis is present, free hydrochloric acid may be absent entirely.

The following are two typical analyses:

1. Gastric ulcer near the pylorus :

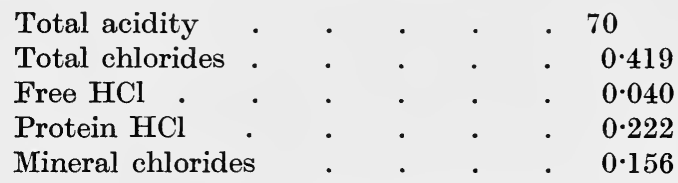

2. Gastric ulcer at the cardiac end of the stomach :

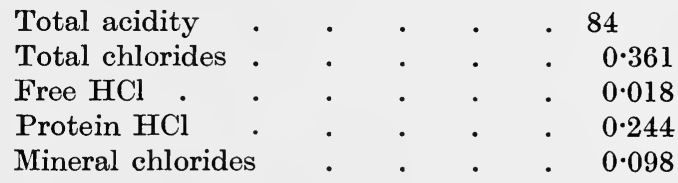

When a gastric ulcer is near the pylorus the clinical differentiation from duodenal ulcer often is impossible.

The following is a good illustrative case of gastric ulcer :

A. W., a hospital nurse aged thirty. Patient has suffered from indigestion for many years. She dates her present illness to seven years ago, when she began to have attacks of severe pain and a feeling of fullness after meals. The onset of pain varied between ten minutes and three hours after food. Until a year ago the attacks occurred at irregular intervals, and sometimes were accompanied by vomiting. 
She has never had hæmatemesis. For the last year the attacks have come on regularly two hours after every meal, and almost invariably have been accompanied by vomiting. Patient has lost flesh considerably and is anæmic. Diagnosis : gastric ulcer. (See Analysis No. 2 on p. 121.)

At operation a large ulcer was found near the cardiac end of the lesser curvature, causing slight constriction of the stomach. An anterior gastro-jejunostomy was performed, and the patient was completely relieved of all her symptoms.

The following case illustrates the difficulty of differentiating gastric ulcer, near the pylorus, from duodenal ulcer, as the symptoms suggested duodenal rather than gastric ulcer.

H. B., aged forty-nine. For the last thirty years patient has suffered more or less from indigestion, water-brash, occasional vomiting, flatulence, and pain, coming on three or four hours after food. He usually has three or four attacks in the course of a year, and is comparatively well in the intervals. During the past three years, the attacks have been much more severe than formerly. He has never had hæmatemesis or melæna. The pain comes on three or four hours after food, and is relieved by water-brash, or by vomiting. Usually he is wakened up at night every four hours by pain or flatulence. For the last six months he has been getting steadily worse, and has lost nearly two stone in weight. He is now extremely emaciated. (See Analysis No. 1 on p. 121.)

At the operation a large indurated ulcer was found in the lesser curvature, about two inches from the pylorus. A posterior gastro-jejunostomy was performed, and the patient is now in excellent health, and has put on a stone and a half in weight.

Occasionally hæmorrhage is the most prominent symptom of gastric ulcer.

A lady, sent to me by Dr. Soltau Fenwick, had a severe hæmatemesis seven months before I saw her. She brought 
up about a pint of blood, and her hæmoglobin was reduced to 21 per cent. A month later she had another, slighter hæmatemesis. Shortly afterwards she began to have pain in the stomach, which did not have any constant relation to food. A month later she had another attack of hæmatemesis and melæna, preceded by ten days of severe indigestion. Latterly, the pain had wakened her up at night. The patient was extremely anæmic.

Analysis of gastric contents :

Recovered one hour after test-meal-90 c.c. Good motility.



Diagnosis : gastric ulcer.

On opening the abdomen I found a puckered ulcer in the stomach near the pylorus, and performed a posterior gastrojejunostomy. The patient was completely relieved.

Diagnosis. - It must be admitted that often the diagnosis of gastric ulcer is very difficult. Many cases of supposed gastric ulcer are really instances of appendicular gastralgia. The symptoms are gastric, but the lesion is in the appendix. In other cases the symptoms are due to chronic gastritis, or to gall-stones.

When a gastric ulcer is close to the pylorus, differentiation from duodenal ulcer may be impracticable, as in the illustrative case given (p. 122).

The points in the diagnosis are:

(i) The chronicity of the disease.

(ii) The periodicity of the disease.

(iii) The time relation of the pain to food, usually one hour after food, but often less than this period.

(iv) The diminution of free hydrochloric acid. 
(v) The control of the pain and other symptoms by the administration of alkalis.

Differential Diagnosis.-Gastric ulcer has to be distinguished from duodenal ulcer, gall-stones, appendicular gastralgia, syphilis of the stomach, Bright's disease, gastroptosis, and intestinal stasis.

As pointed out already, a gastric ulcer near the pylorus may be indistinguishable clinically from a duodenal ulcer. As a rule, in gastric ulcers near the pylorus the onset of pain is from one to two hours, and in duodenal ulcer from two to three hours, or even longer, after food. Occasionally, however, in gastric ulcer, the interval between the taking of food and the onset of pain is several hours. The pain in duodenal ulcer is referred to the middle line, sometimes to the right of it. In gastric ulcer the pain is usually in the middle line, but not infrequently to the left of it. If there be radiation of the pain, it is usually to the right in duodenal ulcer, to the left in gastric, unless the ulcer be near the pylorus, when the radiation may be to the right.

Hæmorrhage is more often evidenced by hæmatemesis in gastric ulcer, and by melæna in duodenal ulcer.

In gastric ulcer, hyper-acidity, if present, is not marked; more often there is hypo-acidity. In duodenal ulcer hyperacidity is much more common than hypo-acidity.

In gastric ulcer hyper-chlorhydria is exceptional, unless the ulcer be near the pylorus. In duodenal ulcer hyperchlorhydria is the rule, hypo-chlorhydria the exception. The hyper-tonic stomach so common in duodenal ulcer rarely is seen in gastric ulcer. Hyper-secretion is more common in duodenal than in gastric ulcer.

From gall-stones in the early stage, gastric ulcer often is hard to differentiate. Usually, close attention to the history will determine the diagnosis. In gall-stones the attacks of 
pain or discomfort after food are slight, occur at irregular times, sometimes after food, sometimes not, are accompanied by much flatulence, often by a " catchy" pain on inspiration. The attacks pass off as suddenly as they have come, and are uninfluenced by drugs, or by diet.

In the later stage of gall-stones, the attacks of pain are more severe, and, like the milder attacks, come and go suddenly, bear no relation to food, radiate through to the back, and are sometimes of an agonising intensity never seen in cases of simple gastric ulcer. In gall-stone disease the gastric contents vary little from the normal, any change being in the decrease of hydrochloric acid and lowering of the total acidity. Hyper-secretion is sometimes present, but it is not common.

Chronic disease of the appendix frequently gives rise to gastric symptoms (appendicular gastralgia). The pain is more indefinite, discomfort rather than pain, referred to the epigastrium, but less definitely to any particular spot. It follows quickly on the ingestion of food, and the kind of food makes little difference. Even milk causes discomfort. Sometimes the pain radiates to the right iliac fossa. Exercise and physical exertion bring on the pain in a manner that is rare in gastric ulcer. The symptoms are very chronic, often last for years with little tendency to get worse. In gastric ulcer, if untreated, usually there is progressive aggravation of the distressing symptoms.

The pain of appendicular gastralgia is uninfluenced by alkalis. Occasionally there is some tenderness over the appendix region. The gastric findings are those of chronic gastritis, much mucus, bad motility, diminished chlorides, absence or great diminution of free hydrochloric acid, and, as a rule, hyper-acidity, partly due to the increase of volatile acids. Commonly, there is hyper-secretion, and delayed evacuation of the stomach. 
The differentiation of a simple ulcer from syphilis of the stomach is difficult, and at the same time of the utmost importance. If syphilis be the cause of the symptoms, the use of anti-syphilitic remedies is imperative, if the patient is to be relieved. Pain after food, and vomiting, are symptoms common to both complaints. If there be a history or evidence of syphilis, the possibility that the gastric symptoms are due to this cause must be considered.

In syphilis of the stomach hæmatemesis is not common, but there is often profound anæmia. The cachectic appearance of the patient is suggestive. The extreme severity of the pain, and its obstinacy to ordinary treatment, are the chief clinical features of gastric syphilis. Gastric analysis shows an extreme degree of chronic gastritis, great diminution of chlorides, low total acidity, and absence of free hydrochloric acid. In cases of doubt a Wasserman's test should be made.

In Bright's disease, digestive disturbances with anæmia and emaciation may suggest ulcer. The less severe character of the pain, the presence of albumen in the urine, the condition of the heart and vessels, usually enable a correct diagnosis to be made.

Gastric ulcer should not be confounded with gastroptosis. In gastroptosis usually there is hyper-acidity and normal or slightly diminished free hydrochloric acid. Discomfort rather than pain is the chief complaint. Contrary to what obtains in gastric ulcer, the patient usually is more comfortable on a solid diet. Distension of the stomach with air reveals the displacement of the viscus.

In some cases of intestinal stasis the dyspeptic symptoms are so marked as to suggest gastric ulcer. Pain or discomfort, and tenderness over the stomach, nausea or vomiting, together with the pallid, emaciated appearance of the patient, present a clinical picture suggesting gastric ulcer. 
There is no doubt that not infrequently gastro-jejunostomy has been performed in these cases, and naturally has not been followed by relief. The signs of intestinal stasis have been described admirably by Mr. Arbuthnot Lane.

The main indications of intestinal stasis are :

1. The stained and inelastic condition of the skin.

2. The feebleness of the circulation, and the lowering of blood-pressure.

3. The subnormal temperature.

4. The drowsy and apathetic condition of the patient.

5. The delay in the passage of the fæces through the large bowel, as demonstrated by X-rays, or by the administration of charcoal.

In intestinal stasis, symptoms of auto-intoxication are marked, in gastric ulcer absent. In intestinal stasis, as a rule, the cæcum is markedly distended and gurgling is felt readily. Tenderness is not limited to the epigastrium, but is present in both iliac fossæ, often all over the abdomen.

In intestinal stasis the symptoms usually are relieved at once by rest apart from diet; in gastric ulcer it is the diet, not the rest, which influences the pain.

In intestinal stasis there is marked diminution of all the constituents of the gastric juice.

\section{REFERENCES}

Bardeleben, Verschir's Archiv, v. p. 250.

Bolton, C., Proc. Roy. Soc. Med., ii. No. 2 (Path. Sect.), p. 54.

Brinton, Ulcer of the Stomach, 1854.

Cruveilhier, Anatomie Pathologique, 1829.

Debove and Remond, Traité des Maladies de l'estomac, p. 255.

Dieulafoy, Clinique Médicale de l'Hôtel Dieu, 1899, pp. 1-62.

Dittrich, Prager Vierteljahrschr., vol. vii.

Fenwick, Soltau, Ulcer of the Stomach, 1900.

Lane, Arbuthnot, Brit. Med. Jour., 1912, i. p. 991.

Murchison, Path. Soc. Trans., xvii. p. 145.

Rokitanski, Oesterreich. Med. Jahrb., 1839, Bd. xviii. p. 184.

Welch, Peper's System of Practical Medicine, 1885, ii. p. 482. 


\section{CHAPTER IX}

\section{DUODENAL ULCER, ITS PATHOLOGY, SYMPTOMS, AND DIAGNOSIS}

Duodenal ulcer occurs in two forms, the acute and the chronic. Usually the acute ulcer is secondary to extensive burns of the skin, more rarely to acute appendicitis. It occurs also as a complication of septicæmia, pyæmia, or acute nephritis, and in the course of the specific fevers. Probably such ulcers are toxic in origin; as a rule they cause no symptoms, and are recognised only on post-mortem examination. In other cases hæmorrhage or perforation ensues and hastens the death of the patient. When these complications result, the patients are already so seriously ill, that operation is not likely to prove of service. Therefore acute duodenal ulcer is of little surgical importance.

Chronic Ulcer of the Duodenum is by far the commoner and more important variety. It is situated most commonly on the anterior wall of the duodenum within three-quarters of an inch of the pylorus. Sometimes the ulcer extends up to, and is continuous with, the pylorus. Dr. W. J. Mayo was the first to call attention to the importance of the "pyloric vein" as a surgical landmark. By observing the relation of the ulcer to this vein, whether the ulcer is gastric or duodenal, may be determined without difficulty.

On the operation-table the site of the ulcer is indicated by injection or mottling of the peritoneal coat. In ulcers of long standing, the base may appear as a yellowish-white area with a depressed and puckered centre. On palpation the ulcer feels hard, like a ring of cartilage. If the wall of 
the bowel be pushed in front of the examining finger the "crateriform" shape of the ulcer often can be recognised clearly. In the majority of cases there are no adhesions, but in other instances the base of the ulcer may be adherent to the liver, gall-bladder, or to a tag of omentum-Nature's protection against acute perforation.

Cicatrisation of the ulcer may produce considerable narrowing of the duodenal lumen. Sometimes the stricture takes an annular form, dividing the first portion of the duodenum into two compartments (" hour-glass duodenum "). Dr. W. J. Mayo and Sir Berkeley Moynihan have recorded instances of double stenosis (" trifid duodenum ").

How long a duodenal ulcer may exist before its presence can be detected by inspection and palpation on the operation table, we do not know. It is possible, indeed probable, that in the early stages an ulcer may be present which cannot be felt. Some years ago I had an interesting case bearing on this point. A lady, aged forty-two, had had severe vomiting, hæmatemesis, and melæna. On opening the abdomen, I found an enormously large appendix measuring $3 \frac{1}{2}$ inches in diameter, and containing three large, foulsmelling concretions. I explored the stomach and duodenum carefully, but could find no evidence of gastric or duodenal ulcer. The symptoms recurred, and she had further severe attacks of hæmatemesis and melæna. A year later I reopened the abdomen, and on this occasion found a wellmarked indurated duodenal ulcer adherent to the liver. I have little doubt that, in this case, a superficial ulcer, which could not be felt, existed at the time of the first operation. The ulcer persisted, and in due time became indurated, so that at the second operation it was palpable as well as recognisable by sight.

More than one ulcer is present in about 15 per cent. of the cases. When two ulcers exist, usually they are opposite 
each other, suggesting that the second ulcer is due to infection from the first. Such ulcers have been termed " contact ulcers."

Formerly, it was believed that gastric ulcers were far more common than duodenal, the relative frequency being given variously as from five to ten gastric to one duodenal.

Many ulcers which were really duodenal were classed as pyloric and therefore gastric. Now that we can distinguish between gastric and duodenal ulcers, by observing the situation of the ulcer in relation to the pyloric vein, we know that duodenal ulcer is as frequent, if indeed not more frequent, than gastric ulcer.

In 200 patients operated on at the Mayo clinic, 60 ulcers (31 per cent.) were situated in the stomach and 119 (61 per cent.) in the duodenum.

In my own series of patients, in 60 per cent. the ulcer was in the duodenum, in 34 per cent. in the stomach, while in 6 per cent. there was an ulcer both in the stomach and in the duodenum.

As to the sex incidence: at the same clinic, duodenal ulcer was found 77 times in men and 23 times in women. Sir Berkeley Moynihan gives 73.6 per cent. in men, and 26.4 per cent. in women. In my own series, 80 per cent. of the duodenal ulcers have been in men and 20 per cent. in women. It is interesting to note that the sex incidence of cholelithiasis is just the opposite of this, namely 76 per cent. in women to 24 per cent. in men.

Ætiology.-The cause of duodenal ulcer is not ascertained as yet. Sir Berkeley Moynihan, in his classical work on duodenal ulcer, does not even discuss the ætiology.

In operating for duodenal ulcer I have been struck with the frequency with which there is coexisting appendicular disease. I have found that obvious disease of the appendix is present in 66 per cent. of the cases in which, at operation, 
I have found duodenal ulcer. This association seems to me to be so frequent that it is not a mere coincidence, and I have suggested elsewhere, that latent appendicitis is one cause of duodenal ulcer. The evidence of this is at present only presumptive. It is highly probable, in some instances, that gastric and duodenal ulcers are toxic in origin, and I think it is possible that duodenal ulcer is the result of toxic absorption from the appendix.

It is now established clearly, that appendicular disease does produce definite gastric symptoms, a condition for which I have suggested the term " appendicular gastralgia." Some years ago I pointed out that in these cases the gastric mucosa often shows an extreme degree of erosion. It is possible that this condition is the prelude to definite ulceration of the stomach or duodenum, the sequence of events being: appendicular disease; secondarily, erosion of the gastric mucosa; then superficial ulceration (the so-called "medical ulcer"); and later a demonstrable indurated ulcer. The brilliant work of Mr. Arbuthnot Lane has thrown new light on obscure abdominal symptoms, and it is quite possible that intestinal stasis may be another factor in the ætiology of duodenal ulcer.

Clinical History.-In chronic duodenal ulcer three features are very definite and constant: the chronicity of the disease, the periodicity of the attacks, and the well-being of the patient in the intervals between the attacks. The duration of the disease is marked by years rather than months. In my own series of cases the average duration of symptoms is eight years. In reply to the query, "How long have you been ill?" it is not uncommon for the patient to reply, "As long as I can remember."

The onset of the symptoms is insidious; the patient notices that he has a feeling of discomfort two or three hours after the principal meals. Thus, a common history is, that 
the discomfort is experienced about four or five o'clock in the afternoon, and continues until the next meal be taken. If dinner be taken in the evening, the discomfort may recur about ten o'clock. In the early stages of the ulcer the discomfort may be trifling, and little importance is attached to it, but it increases gradually, until it merits the description of pain. The frequency of its return likewise increases, so that after every meal there is the same sequence of events : pain coming on within two or three hours, continuing up to the time of the next meal, and relieved temporarily, by the taking of food. To the patient it appears as if the pain were due to want of food, not to the food taken some hours previously. For this symptom Sir Berkeley Moynihan has suggested the apt description " Hunger pain."

In the later stages of the disease a prominent symptom is pain coming on in the early hours of the morning. The patient goes to sleep in comfort, but is awakened about two or three o'clock, by a burning pain in the epigastrium. This early-morning pain is symptomatic of hyper-secretion.

A most marked feature of duodenal ulcer is the intermittence of the symptoms. They occur from time to time in attacks, and during the intervals between the attacks the patient may be in excellent health, and be able to enjoy an ordinary diet without sign of discomfort. The attacks may last a few days or continue for weeks. Often there is a definite cause for the onset of the attack-“" a chill," some indiscretion in diet, or a change of climate.

One of my patients, who had had symptoms for nearly thirty years, told me that during his undergraduate days, while he was at Cambridge, he was always perfectly well, but directly he came to London for his vacation, he had an attack which continued as long as he remained in town.

Sometimes the intervals between the attacks are measured by years, so that the patient may think he is quite cured, 
until he is disillusioned by a return of his trouble. Gradually the severity, or frequency of the attacks, or both, increases, and the intervals of good health become shorter. Even between the attacks the patient suffers from discomfort after food, accompanied by flatulence and belching of gas. Up to this point the patient may have maintained his normal weight and natural energy. But with increasing inability to take normal diet without pain, he restricts his food supply, begins to lose weight, and suffers in mental and bodily vigour.

\section{SYMPTOMS}

Pain.-The pain in duodenal ulcer usually is referred to the epigastrium. Radiation to other regions is uncommon. It is burning and gnawing in character, in the early stages, discomfort rather than pain, but in the later stages it may be very severe. Indeed, I have known patients in whom the pain was of such severity, that they were, for a short time, " doubled up" with it. This, however, is exceptional. My experience is that pain comes on rather sooner after a meal than is generally taught. I should put the usual time of onset at about two hours, sometimes it is delayed for five or even seven. In a few instances patients state that the pain comes on immediately after, or within half an hour to an hour of taking food. In some of these cases adhesions are present, or there is puckering of the duodenum, but this is not the case invariably. Possibly, in the early stages, the onset of the pain was not so rapid, but the later and more immediate pain has impressed itself so much on the patient's mind, that the memory of the earlier sequence of events has been obliterated. The pain of duodenal ulcer can be relieved, temporarily at least, by the administration of bicarbonate of soda.

Vomiting.-In 60 per cent. of my cases there is a history 
of vomiting. Some writers state that vomiting is rare in duodenal ulcer, except when there is duodenal stenosis. With this statement I do not agree. I think that vomiting is not uncommon, but is rarely due to duodenal stenosis. It is due, either to reflex emptying of the stomach, or to reverse peristalsis of the duodenum, beginning at the site of the ulcer. Some years ago I suggested that the vomiting might be due to reverse peristalsis, and my hypothesis was characterised as being very improbable. Since then, on more than one occasion, by means of the X-rays, I have been able to see this reverse peristalsis commencing at the site of the ulcer, and, as it were, shooting the duodenal contents back into the stomach. Further evidence of such regurgitation is the observation, that in duodenal ulcer, it is not uncommon to find small quantities of bile in the fasting stomach.

As vomiting was present in 60 per cent. of my cases, and organic obstruction, as verified by operation, in only 20 per cent., it is clear, that in a considerable proportion the vomiting is not due to organic stenosis.

Usually the amount vomited is not large in quantity; the emesis occurs two or three hours after food, and is followed by immediate relief from pain. When there is stenosis due to cicatrisation of the ulcer or duodenal adhesions, the patient may vomit large quantities, and there is marked food retention.

Hæmorrhage.-Hæmorrhage, as evidenced by hæmatemesis or melæna or both, occurred in 22 per cent. of my cases. It is usually a late symptom. Contrary to the accepted teaching, I believe that in the majority of cases hæmorrhage, when it occurs, comes, not from the ulcer, but from the mucous membrane of the stomach. The hæmorrhage is out of proportion to the extent and erosion of the duodenal ulcer. In such cases I have seen the whole of the gastric mucosa studded with numberless bleeding points. The 
interior of the stomach is in the condition described as "weeping blood." I have several microscopic sections of the gastric mucosa in cases of duodenal ulcer, in which blood clearly can be seen escaping between the epithelial cells. Probably this condition of the mucosa is due to the irritation of hyper-acid gastric juice, or to the continual presence of gastric juice in the fasting stomach. In support of this view it is an interesting fact, that marked hyperchlorhydria was present in all but one of my patients who had had recent hæmorrhage.

As I have said before, as a rule, hæmorrhage is a late symptom, but occasionally an alarming hæmorrhage is the first symptom that attracts the attention of the patient. In these cases careful questioning will always elicit the fact, that there has been previous indigestion.

Examination of Gastric Contents.-Very often (in 50 per cent. of my cases) duodenal ulcer is accompanied by hypersecretion, so that if a tube be passed in the early morning, from 20 c.c. to 50 c.c. of clear acid fluid is drawn from the stomach. That this is hyper-secretion, and not the result of obstruction, is proved by the circumstance that the fluid drawn off contains no food-remains, and that in these cases the stomach empties itself within the normal limit of time, and that the amount of the test-meal withdrawn is not greater than the average in health.

In my experience duodenal obstruction, due to adhesions or cicatrisation, is present in 20 per cent. of the cases. When this is so, food-remains are found in the stomach in the morning, together with a considerable amount of fluid, sometimes as much as 500 c.c. The amount, too, of gastric contents recovered after a test-meal is in excess of the normal.

I believe that at some period of the disease, hyperchlorhydria is present in most cases of duodenal ulcer. In 
some instances hyperchlorhydria is present during, or shortly after, the attacks, but between the attacks the free hydrochloric acid is normal, or a little above normal. Sometimes the hyperchlorhydria is latent, that is, there is excess of free hydrochloric acid, but its presence is masked by the regurgitation of bile from the duodenum into the stomach, which, as I have pointed out already, occurs, not infrequently, in cases of duodenal ulcer. In 52 per cent. of my cases there was hyperchlorhydria, and hyper-acidity in 70 per cent.

In a few cases (10 per cent. in my series) no free hydrochloric acid is found on examination of a test-meal.

I am of opinion that in these cases the absence of free hydrochloric acid is secondary to intestinal stasis, or to appendicular disease. I think we may state, that as a general rule, hyperchlorhydria accompanies duodenal ulcer, and that it is uncommon to find the free hydrochloric acid diminished.

Diagnosis.-In the vast majority of cases of duodenal ulcer, the diagnosis presents no difficulty. The history alone is sufficient. So far as diagnosis is concerned physical examination of the patient is of secondary importance.

Investigation of the gastric motility and secretion should never be omitted. It is true that in the majority of cases it is valuable only as a confirmation, but in a number of cases it is of the utmost value in the differential diagnosis.

As regards the history - as has been well emphasized by Dr. Christopher Graham-it is not the periodicity of the attacks nor the chronicity; both these characteristics are present in appendicular gastralgia; it is (i) the time of the onset of the pain and other symptoms, (ii) the regularity of the pain and other symptoms, (iii) the means by which the pain and other symptoms are relieved.

The important points in the diagnosis of duodenal ulcer are : 
1. The definite relation of the pain to food.

2. The length and constancy of the time interval.

3. The recurrence of the attacks.

4. The chronicity of the disease.

5. The intervals of good health between the attacks.

6. The relief of pain by alkalis.

7. The presence of hyperchlorhydria, hyper-acidity, and hyper-secretion.

The following is a good illustrative case of duodenal ulcer : A gentleman aged forty-three years began to suffer from attacks of discomfort in the abdomen twenty-seven years before I saw him. The attacks came on at intervals, and lasted two to three weeks at a time. During the attacks the discomfort came on regularly at five o'clock in the afternoon and lasted until dinner-time. He did not suffer much from flatulence. While he was out of London he was free from discomfort, but directly he came back, invariably he had an attack. Ten years before I saw him the attacks became more severe, so that instead of discomfort he had pain, which, during the attacks, came on from two to two and a half hours after food. For five years the attacks had recurred at longer intervals, but had been more severe, and the pain more continuous. Latterly, the pain had come on at any hour of the day or night; often he was awakened during the night by it, and was accustomed to take some bread and butter, after which he obtained relief for about an hour. Food relieved the pain immediately. $\mathrm{He}$ had never had any vomiting, but during the attacks had acid eructations and a desire to vomit. Between the attacks he was always absolutely well. Almost invariably the attacks were brought on by some definite cause, such as getting a chill, by worry, or by taking indifferent whisky. There was no tenderness anywhere in the abdomen.

The analysis of the gastric contents was as follows : 

60 c.c.

In the stomach in the morning after ten

Recovered one hour after test-meal-120 c.c motility, some mucus, normal colour.

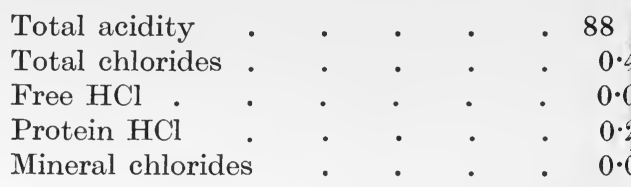

Diagnosis : duodenal ulcer.

At the operation I found a puckered, cicatrised ulcer on the anterior wall of the duodenum close to the pylorus. The appendix was chronically inflamed and was removed, and a posterior gastro-jejunostomy performed. The patient was completely relieved, and now feels better than he has done for many years.

Occasionally hæmorrhage is the first symptom that attracts the patient's attention.

A man aged forty-three (admitted under the care of my colleague, Dr. Soltau Fenwick), noticed some years before admission that he was passing black motions. Cross-examination elicited the fact that for many years previously he had suffered from indigestion. Five years before admission he began to suffer from severe pain in the stomach three hours after food, sometimes accompanied by vomiting. A few days before admission he began to suffer from vomiting one and a half hours after food, and again he noticed that his motions were black. On admission he had some melæna, which continued with slight intermission for three weeks. He became extremely anæmic, but under medical treatment gradually improved.

The following is the result of the gastric analysis :

In the stomach in the morning after ten hours' fast-nil.

Recovered one hour after test-meal-165 c.c. 
The contents were normal in colour, good motility, not a marked amount of mucus present. Volatile acids, $6 \cdot 4$.

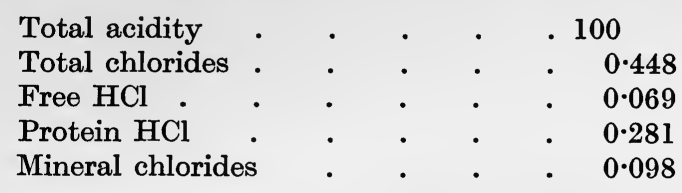

Differential Diagnosis.-The conditions which present the greatest difficulty in diagnosis are, gastric ulcer, gall-stones, appendicular gastralgia, and partial obstruction of the large bowel.

The differential diagnosis of gastric and duodenal ulcer has been discussed already (p. 124).

In gall-stones the pain has no relation to food, appears suddenly without warning, and disappears as suddenly as it comes. It is not relieved by food or alkalis. Vomiting gives no relief. The pain may be exceedingly severe (biliary colic), and cause spasm of the diaphragm. The pain is epigastric and radiates to the back and right scapula, or may surround the right half of the chest like a girdle.

Cholelithiasis in my experience is never accompanied by hyperchlorhydria; any change in the gastric contents is in the direction of diminished rather than of increased free hydrochloric acid, evidence of a secondary gastritis.

Vomiting is common in cholelithiasis, but unlike the vomiting in duodenal ulcer, is not followed by any abatement of the pain. In the early stage of gall-stone cases often there occur mild dyspeptic attacks, that is, irregular attacks of slight discomfort after food, accompanied by flatulence, sometimes coming on after food, sometimes not, appearing suddenly, disappearing as suddenly as they come. The irregularity of the onset of these attacks, the absence of any constant relation to the ingestion of food, render these 
attacks very suggestive. In such cases gastric analysis will reveal little or no deviation from the normal.

In appendicular gastralgia the symptoms sometimes mimic duodenal ulcer with remarkable fidelity. There is often the same history of recurrent attacks, the same chronicity, and in rare instances, appendicular gastralgia is accompanied by hyperchlorhydria. Even "hunger pain " may be a symptom of appendicular disease, or to put it more accurately, I have met with cases in which "hunger pain" was a symptom, and in which there was a diseased appendix, but no discoverable duodenal ulcer.

It is possible of course, that in such cases an early duodenal ulcer may be present which is confined to the mucous coat, and so not palpable at operation. (See case on p. 129.)

The points which aid us in distinguishing the two diseases appear to me to be :

First, in duodenal ulcer, usually the patient is free from symptoms between the attacks; in appendicular gastralgia, even between the attacks, the patient suffers from flatulence and discomfort after food.

Secondly, the radiation of the epigastric pain towards the lower part of the abdomen is highly suggestive, if not diagnostic, of appendicular trouble.

Thirdly, the discovery of tenderness over the appendix sometimes throws light on a doubtful case.

Fourthly, the pain of duodenal ulcer is more severe than that due to appendix disease. Sometimes, however, the sufferers from appendicular gastralgia have attacks which compel them to lie up. In some instances these attacks are accompanied by a rise of temperature which suggests at once the true cause of the symptoms. In other cases the temperature remains normal throughout the attack.

Fifthly, duodenal ulcer usually is associated with hyper- 
chlorhydria ; in appendicular gastralgia the free hydrochloric acid is diminished or absent.

With regard to the appendix cases in which there is hyperchlorhydria, my impression is, that in duodenal ulcer hyperchlorhydria is associated with much more severe pain than is the case with hyperchlorhydria in appendicular disease.

On several occasions I have met with early cases of malignant growth of the large intestine in which the history suggested duodenal ulcer. The patient's chief symptom has been, pain coming on from two to three hours after food, and relieved by vomiting. A curious feature of some of the cases has been the periodicity of the attacks. In the cases which I have seen, cross-examination invariably has elicited the fact, that during the attacks the pain comes on quite independently of food and is not relieved by taking more food. Such cases illustrate the importance of a carefully taken and accurate history.

\section{REFERENCES}

Graham, C., Illinois Med. Jour., Aug. 1909.

Mayo, W. J., Annals of Surgery, June 1907.

Mayo, W. J., Jour. Amer. Med. Assoc., Aug. 15, 1908, 41, p. 556.

Moynihan, Duodenal Ulcer, 1910.

Paterson, H. J., The Lancet, 1911, ii. p. 97. 


\section{CHAPTER X}

\section{THE TREATMENT OF GASTRIC AND DUODENAL ULCERS}

The Treatment of Gastric Ulcer.-To Rydygier belongs the credit of having performed the first operation for the cure of a gastric ulcer. In the year 1881 he resected a gastric ulcer from the posterior wall of the stomach ; his patient recovered and was completely cured. In the year 1893 Doyen substituted gastro-jejunostomy for excision of the ulcer. This surgeon and Talma of Berlin, independently, had come to the conclusion that some form of pyloric stenosis, probably spasmodic, is the chief factor in maintaining the hyperacidity which prevents the healing of gastric ulcers. Gastrojejunostomy was soon adopted as the operation of choice for gastric ulcer. Rydygier, however, continued to advocate excision, in the belief that the scar of an ulcer may become the seat of carcinoma. The results of gastro-jejunostomy, when performed for pyloric stenosis, were brilliantly successful. To some extent, gastro-jejunostomy for ulcers without stenosis proved disappointing. It was thought that the failures were due to the patency of the pylorus. Some surgeons, therefore, advocated that when gastro-jejunostomy was performed in such cases, the pylorus should be excluded deliberately by suturing, a measure based on erroneous pathology and little calculated to prevent closure of the anastomotic opening. Doubtless some patients were unrelieved on account of mistakes in technique, but probably many more were no better because gastro-jejunostomy was performed without proper indication. The operation was 
performed on a clinical diagnosis, and not on the evidence found on the operation-table. Before operation a diagnosis of gastric ulcer was made, at the operation no ulcer was found. It was assumed that the symptoms were due to an ulcer of the mucous coat, without induration, and so not palpable. Gastro-jejunostomy was performed, and a lesion in the appendix or elsewhere was undiscovered and left untreated. The patient was no better, the case being recorded as an operative failure, whereas it was really, as Dr. W. J. Mayo puts it, " a mistake both in diagnosis and operation."

During the last few years the treatment by excision has been revived, but it is advocated for different reasons than formerly. It is argued, first, that gastro-jejunostomy is a drainage operation, and therefore its performance in cases of ulcer away from the pylorus is useless, as the food continues to pass through the patent pylorus and not through the new opening. In other words, the drain does not act. I have already expressed my firm conviction that gastrojejunostomy is a physiological, and not a drainage operation. Whether the food passes out through the pylorus, or through the anastomosis, is of no consequence. I have performed gastro-jejunostomy many times in cases of ulcer of the lesser curvature, or ulcer at the cardiac end of the stomach, and have kept in touch with all the patients who had ulcers in these positions, and I can say, without hesitation, that the results have been more satisfactory than the results of gastrojejunostomy for other ulcers. Every one of these patients has been completely relieved.

Secondly, with regard to callous ulcers at the pyloric end of the stomach, it is said that these should be excised, because they are apt to degenerate into cancer. It is stated that a large proportion of gastric carcinomata have their origin in simple ulcer. The treatment by resection, in selected cases, has received the imprimatur of so great an 
authority as Dr. W. J. Mayo. The experience at St. Mary's Hospital (Rochester) is that in no less than 71 per cent. of the cases of gastric cancer, the starting-point is a gastric ulcer. From personal knowledge I have such a high admiration and respect for all the work of the Mayo clinic, that I feel it is almost presumptuous to disagree with any conclusions arrived at by Dr. Mayo.

I do venture to think, however, that the evidence, on which the existence of ulcer previous to malignant disease is based, is far from conclusive. If the view that cancer be grafted on ulcer be correct, gastro-jejunostomy must be performed often on ulcers which already are malignant. Why is it, then, so rare an event for a patient to die from cancer, after gastro-jejunostomy for supposed simple ulcer? When a mistake is made, it is more likely to be a mistake in the opposite direction. It is the supposed malignant case which proves innocent, not the supposed simple case which proves malignant.

Most surgeons who have had much experience in gastric surgery have met with cases in which there is a hard mass at the pylorus, tightly bound down to the pancreas and other structures, with enlarged glands along the lesser curvature, a condition of things rendering resection absolutely impracticable. A gastro-jejunostomy is performed as a palliative operation, in the hope that the patient will gain a few months' comfort. To the surprise of the surgeon the patient has regained perfect health, and lived so long that the existence of cancer is negatived. Yet if the grafting hypothesis be correct, it is in just such a case as this, that one would expect death eventually to occur from cancer.

I have had a fairly large experience of the after-results of gastro-jejunostomy, both as performed by myself and as performed by others, and I have met with two cases only (one operation performed by myself, and one by another 
Plate XXII.

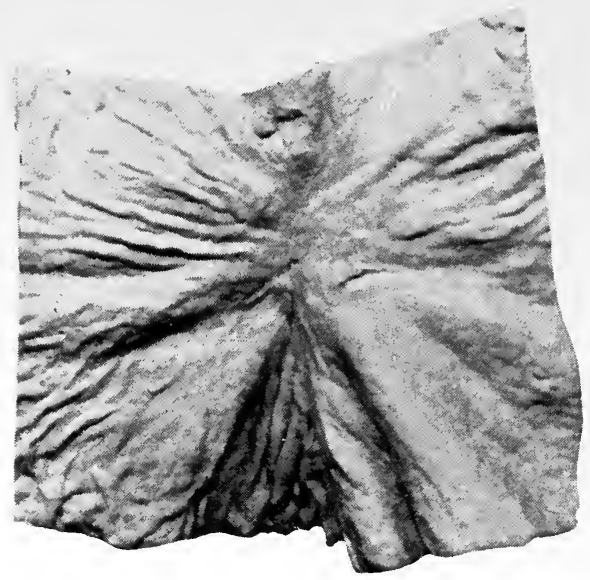

FI(x. 51.-Cicatrix of a chronic ulcer. (Soltau Fenwick.)

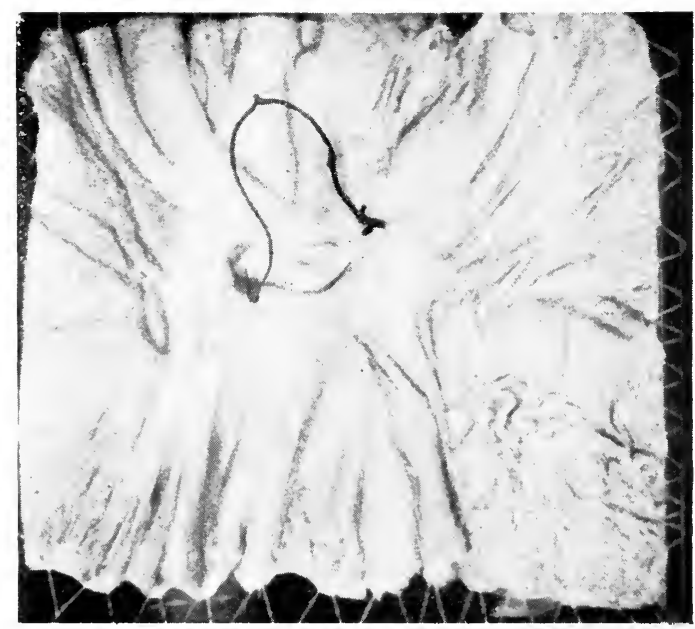

FIG. 52.-Recurrent ulcer in the operation scar, from a man who died of hæmatemesis three months after excision of gastric ulcer. (St. Bartholomew's Hospital Museum, No. 1917b.) 
surgeon) in which, after gastro-jejunostomy for supposed simple ulcer, the patient has died from gastric carcinoma. Professor Kocher has published lately a review of fifty cases of gastro-jejunostomy, in which the periods which have elapsed since operation, varied from two to twelve years. There was no recurrence in any case, and Dr. Kocher concludes with justification that there is therefore no need for resection. Dr. Gressot states that malignant degeneration of surgical ulcers occurs in only $2 \cdot 3$ per cent. of all cases after gastro-jejunostomy. In view of these observations, it appears to me difficult to accept the hypothesis that carcinoma is grafted on simple ulcer in such a high percentage of cases as is taught at the present time.

Personally, I believe that gastro-jejunostomy is preferable to excision, the risk of which is undoubtedly greater than that of the short-circuiting operation.

Further, judging from recorded cases and from my own experience, I have little doubt that relapses are much commoner after excision than after gastro-jejunostomy.

This is just what one would expect, because excision removes the ulcer, but does not influence the causal factor of ulceration. This, in my judgment, is the chief objection to excision.

Gastro-jejunostomy, therefore, should be the operation of choice, because it is a physiological operation, and so alters the gastric contents as to promote healing of the ulcer.

I will now attempt to answer the question, "When should surgery interfere in cases of gastric ulcer?" (Acute ulcer, apart from the complications of gastrorrhagia and perforation, does not call for surgical measures.) The answer to this question appears to me to depend on three considerations :

\section{What is the Natural History of Gastric Ulcer treated Medically?}




\section{The Mortality of the Surgical Treatment Proposed. \\ 3. The Prospect of Cure after Operation.}

1. The Natural History of Gastric Ulcer.-The mortality from gastric ulcer has been estimated variously by different writers, some placing it as high as 50 per cent., and others as low as 20 per cent.

The important point is to determine what proportion of the cases considered to be "cured" remain perfectly well. In my Hunterian lectures in 1906 I gave an analysis of the after-results of a series of cases of gastric ulcer; put in tabular form the results were as follows :

"Not cured:"

Signs of gastric ulcer . . . . . . . . . . 40

Operation subsequently necessary $. \quad . \quad . \quad$. $\quad 5$

Died from gastric disease . $\quad . \quad$. $\quad . \quad$. $\quad . \quad 1$

"Cured" • . . . . . . . . . . . . . 19

"Probably cured". . . . . . . . . . . . $\quad .2$

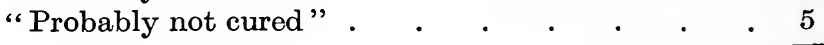

Total . $\overrightarrow{72}$

To avoid any possible exaggeration I will include the five "probably not cured" patients among the "cured." Even so, 64 per cent. of the patients who have been traced have been uncured by medical treatment. Looking at the figures in the most favourable light, at least 31 per cent. of the patients discharged as cured have relapsed, while if the condition of the untraced patients be as unsatisfactory as is that of those who have been traced-and there is no reason to suppose that it would be better-the proportion of relapses would be more than double this figure. When I point out that these patients were in hospital on a strict milk diet for an average period of six weeks, and that they were warned to keep on a milk diet for a further period of six weeks, and to exercise great care for three more months, the significance of such a large proportion of relapses is 
apparent. But the worst has not been said. Although nineteen of the patients are classified as cured, the use of the term is relative, not absolute, and only implies that for the present these patients are free from symptoms of gastric ulcer. One of them, although quite well at the time of inquiry, had been in hospital again for six months, while another was an in-patient for a shorter period. Only seven of the nineteen patients have remained free from any symptoms of gastric ulcer since they left hospital. Of the seventy-five patients whom we have been unable to trace, it is noted that two had been in hospital twice before, and ten once, so that of the untraced patients eleven-i.e. 14 per cent.-were the subjects of relapsing gastric ulcer.

Considering that this series does not include those patients admitted with perforated gastric ulcer, or those admitted with pyloric stenosis, or other sequels of gastric ulcer, I think we may conclude, that so far as hospital patients are concerned, between one-half and two-thirds of the serious cases relapse, and that the proportion of real cures is under 25 per cent.

Gastric ulcer in private practice presents a totally different picture. Private patients, amid more favourable surroundings, bear more readily the necessary restriction of their diet, and relapse is much less common. Consequently operation is required less often for the treatment of an existing ulcer, as for those cases in which, owing to neglect to commence treatment early, cicatrisation of the ulcer has produced pyloric stenosis, gastric adhesions, or hour-glass stomach.

2. The Mortality of the Surgical Proceeding Proposed.My view is that the best method of treating an intractable gastric ulcer is by the operation of gastro-jejunostomy.

The mortality of this operation in skilled hands is not greater than 3 per cent. When it is realised more fully 
that gastro-jejunostomy does not interfere with the metabolic processes of the human body, and that after it patients may live for years in perfect health, doubtless these relapsing cases will be dealt with surgically at an earlier stage, and we may hope reasonably that the mortality will be further diminished. Indeed, there is no reason why the operation of gastro-jejunostomy, if performed before the patient has been worn out and emaciated by months or years of suffering, should be attended with a greater mortality than the operation of appendicectomy during a quiescent interval.

3. The Prospect of Cure by Operation.-Some years ago I collected 278 cases of gastro-jejunostomy from surgical literature and endeavoured to ascertain the after-results. The after-history was obtained in 143 of the cases, and the results are shown in the following table:

"Cured" of gastric trouble :

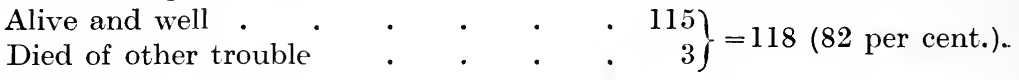

Unsatisfactory cases :

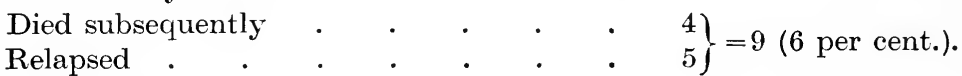

Fairly good results :

Occasional dyspepsia .

Some care in dieting necessary .

$$
\left.\frac{9}{143}\right\}=16 \text { (11 per cent.). }
$$

When it is remembered that these operations were performed by many different operators, and for various conditions, the results must be considered highly satisfactory.

Investigation of my own cases gives a very similar result : 81 per cent. of my patients have been relieved completely; 12 per cent. have been greatly improved, that is, have been completely relieved of their pain, but suffer from occasional discomfort, or have to be careful as to their diet; and 5 per cent. either have been unrelieved, or have had recurrence of their symptoms. 
When should Surgery interfere in Cases of Chronic Gastric Ulcer?-We have now seen that the mortality from gastric ulcer treated medically is at least 20 per cent., and further, that at least 50 per cent. of the so-called "cures" relapse, while probably not 25 per cent. of the patients treated medically are really cured. On the other hand, gastrojejunostomy holds out a great prospect of cure, the proportion of relapses being probably not more than 10 per cent. The immediate risk of operation is about 3 per cent., to which may be added the remote risk from jejunal ulcer of under 2 per cent. I realise fully the danger of advocating operation from comparisons of this sort, but at least these figures suggest that the question of surgical treatment should be considered seriously in every case which fails to yield to medical treatment.

Leube says that if a gastric ulcer be not cured by four or five weeks of treatment, it will not be cured by medical treatment alone. Allowing a wider latitude than this, I would formulate the principles on which to act, as follows: If, after six weeks' complete rest, on a milk diet, a further period of six weeks on a milk diet with comparative rest, followed by three months' careful dieting, the patient be not free from definite symptoms, or if, after apparent cure, the patient have a relapse, operation is probably in the best interests of the patient. The symptoms, however, must be clear and definite. The performance of gastro-jejunostomy in cases of vague gastric disorders, unaccompanied by definite clinical or chemical signs, cannot but lead to the discrediting of a valuable, and in suitable cases, legitimate operation.

The Treatment of Duodenal Ulcer.-In duodenal ulcer the indication for surgical treatment is much more definite than in gastric ulcer. We have evidence that a gastric ulcer may cicatrise and give rise to no further symptoms. In duodenal 
ulcer, such a favourable issue is rare, if it occur at all. My experience is that when once a patient has marked symptoms of duodenal ulcer, recurrent attacks of pain coming on two hours or more after food, with hyperchlorhydria, he will be subject to periodic return of symptoms until surgical treatment be adopted. Dieting, medicinal treatment, and gastric lavage will give relief but will not bring about a cure. There is no doubt that a duodenal ulcer may heal, but this does not necessarily mean relief. The process of cicatrisation often causes stenosis. Even if the ulcer be small, and no narrowing of the duodenum follow the process of healing, the symptoms appear to continue. I have operated in a number of cases in which I found a small healed ulcer, but yet hyperchlorhydria was present and the patient had continued to suffer from typical periodic attacks of duodenal ulcer pain.

Recently, I operated on a young woman who had been in hospital for six weeks, and had been discharged, apparently quite well. She returned three months later with recurrence of her former symptoms. At the operation I found a small scar in the duodenum, not larger than a threepenny piece. There was no stenosis, and no other lesion discoverable. Why in such cases the symptoms persist, is difficult to understand, unless it be that the presence of a scar sets up pyloric spasm. Another factor which renders operation in duodenal ulcer more imperative than in gastric ulcer, is, the greater risk of perforation. On account of the thinness of the duodenal wall, perforation is a more likely complication than is the case in gastric ulcer.

In the early stages medical treatment should have a thorough and extensive trial. Milk diet, rest in bed, the administration of half an ounce of olive-oil three times daily before meals, as recommended by Professor Matthieu, should be persevered with for at least six months. At first, pro- 
bably the ulcer is limited to the mucous coat, and if treated during this stage, we are justified in acting on the belief that it may heal and cause no further trouble, although we do not know whether such is the case or not. (See case on p. 129.)

If, in spite of treatment, the symptoms continue, or if, after apparent cure, relapse ensue, only surgical treatment will give relief.

The following operations may be performed :

1. Gastro-jejunostomy.

2. Excision of the ulcer, if it be small and free from adhesions.

3. Finney's operation.

4. Resection of the portion of duodenum in which the ulcer is situated.

5. Resection of a portion of the duodenum with the ulcer, closure of the distal end of the duodenum, and implantation of the proximal end of the duodenum into the side of the distal portion.

Sir Berkeley Moynihan has advocated excision in suitable cases. This method is open to the same objection as resection for gastric ulcer. It removes the ulcer, but does not influence the hyperchlorhydria.

The results of gastro-jejunostomy for duodenal ulcer are so satisfactory, that I think it is preferable to any method at present in use.

The argument for resection of gastric ulcers, that the ulcer may become the site of cancer, does not apply to ulcer of the duodenum, as cancer of the duodenum is exceedingly rare.

Dr. W. J. Mayo and Sir Berkeley Moynihan recommend that when gastro-jejunostomy is performed, the ulcer should be infolded by two or three mattress sutures, to promote healing and guard against hæmorrhage. This addition can 
do no harm, but whether it is necessary is an open question. I do not adopt it as a routine procedure, although I have used it in some cases. I have never yet had a case of hæmorrhage from the ulcer after gastro-jejunostomy.

\section{REFERENCES}

Aitchison, Brit. Med. Jour., 1904, ii. p. 864.

Armstrong, Montreal Med. Mag., 1896, p. 505.

Barker, Brit. Med. Jour., 1896, ii. p. 1583.

Carle and Fantino, Archiv f. Klin. Chir., 1898, i. p. 217.

Czerny, Archiv f. Klin. Chir., 1888, p. 853.

Dalziel, Glasgow Med. Jour., 1896, p. 302.

Dean, Trans. Med. Soc., 1894, p. 305.

Doyen, Revue de Chir., 1896, p. 568.

Eve, The Lancet, 1908, i. p. 1822.

Gressot, Berliner Klin. Woch., xlix., May 27, 1912.

Hofmeister, Beit. z. Klin. Chir., 1896, ii. p. 351.

Kehr, München. Medicin. Woch., lix., June 18, 1912.

Kocher, Th., Deutsche Zeit.f. Chir., cxv., 1912.

Küster, Archiv f. Klin. Chir., 1894, iv. p. 787.

Lauenstein, Deutsche Med. Woch., 1895.

Leube, Archiv f. Klin. Med., 1897, i. p. 69.

Mayo, W. J., Annals of Surgery, June 1908.

Mikulicz, Archiv f. Klin. Med., lv., 1897, i. p. 84.

Moullin, M., Brit. Med. Jour., 1903, i. p. 954.

Moynihan, Duodenal Ulcer, 1910, p. 9.

Paterson, Gastric Surgery, 1906, p. 47, and Trans. Roy. Med.-Chir. Soc., xc., 1907, pp. 262, 410.

Petersen and Machol, Beit. z. Klin. Woch., xxxiii., 1902, No. 3.

Riedel, Archiv f. Klin. Chir., xcviii., 1912, No. 3.

Robson, Clin. Jour., Mar. 2, 1904.

Russell, J. W., The Lancet, 1904, i. p. 288.

Rydygier, Berliner Klin. Woch., Jan. 1882.

Sears, G., Boston Med. and Surg. Jour., clii., Mar. 30, 1905.

Talma, Berliner Klin. Woch., 1895, No. 15.

Tricomi, Riforma Medica, Feb. 1899. 


\section{CHAPTER XI \\ THE COMPLICATIONS OF GASTRIC AND DUODENAL ULCERS}

\section{PERFORATION}

\section{A. Perforation of Gastric Ulcer}

In the field of human disease there is nothing more tragic than death from sudden perforation of a gastric ulcertragic not only from its suddenness, but from the circumstance that the victim is often young, in the prime of life, and to all outward appearance in the enjoyment of perfect health. The measure of success which has attended the efforts to snatch these victims from the very jaws of death must be accounted one of the greatest triumphs of surgery.

Perforation of the stomach may be acute or chronic.

In acute perforation an ulcer gives way suddenly, allowing the escape of gastric contents. In about 96 per cent. of the cases of acute perforation, the gastric contents escape freely into the general peritoneal cavity, and general peritonitis ensues. In about 4 per cent., owing either to the small size of the perforation, or to the rapid closing of the opening by a plug of lymph or omentum, the escape of gastric contents is limited. Such cases are described as sub-acute. Although the initial symptoms may be as severe as those in which there is general extravasation of gastric contents, the prognosis is more favourable, and the resulting peritonitis may be of very limited extent.

In chronic perforation the destruction of tissue takes place more slowly, so that by the time the whole thickness of 
the gastric wall has been destroyed, the general peritoneal cavity is shut off by a barrier of plastic exudation. Thus, the escape of gastric contents is circumscribed, and the suppuration that ensues almost inevitably, is confined within a limited cavity, forming a peri-gastric abscess.

Although acute perforation does occur in cases of acute gastric ulcer, it is more common in the chronic form, owing either to absence of plastic peritonitis, or to sudden yielding of adhesions which have been formed.

Acute Perforation.-The frequency of perforation has been estimated by various writers at from 5 per cent. to 28 per cent. of all cases of gastric ulcer. In a series of 500 cases $I$ find that perforation occurred in 12 per cent. Perforation proved fatal in 7 per cent. of the cases, a figure corresponding exactly to that given by Welch. Perforation is more common in women than in men $(79.3$ per cent. to 20.7 per cent.). Allowing, however, for the greater frequency of gastric ulcer in women, perforation is relatively more frequent in men. Thus, perforation occurred in 23 per cent. of the male patients, but in only 9 per cent. of the female.

In considering these figures we must bear in mind that until comparatively recently a clear distinction has not been drawn between gastric and duodenal ulcers, and so probably, a number of the ulcers classified as gastric really were duodenal.

Perforation occurs much more frequently in an ulcer of the anterior than of the posterior gastric wall. The importance of this is, that in 90 per cent. of the cases, the perforation is in a position readily accessible for surgical treatment.

Site of Perforation.

112 Consecutive Cases of Perforated Gastric Ulcer.

Anterior Wall.

\begin{tabular}{ccccc}
\hline Pyloric & Middle & Cardiac & Posterior & Not stated \\
Third. & Third. & Third. & Wall. & 3 \\
32 & 33 & 32 & 12 &
\end{tabular}


Plate XXIII.

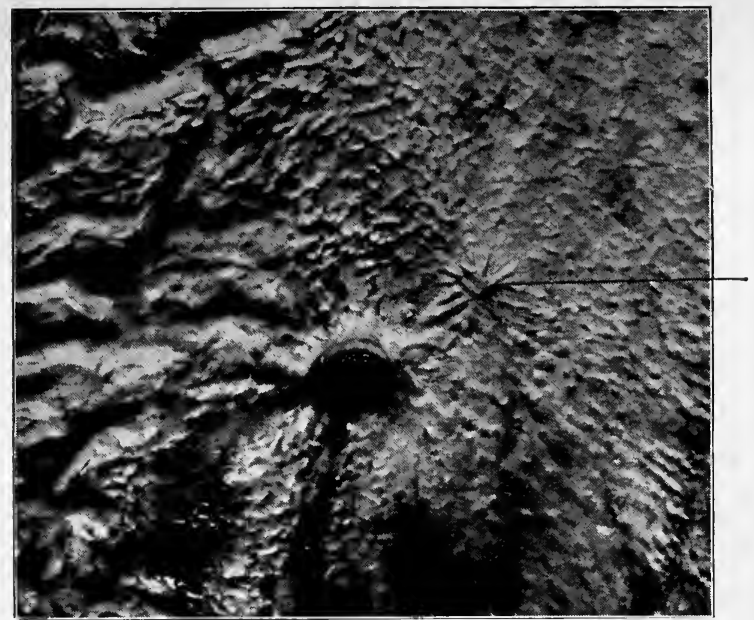

FIG. 53.-Acute perforating ulcer of the stomach in a girl. To the right of the ulcer is a small cicatrix which was discovered by photography. (Soltau Fenwick.)

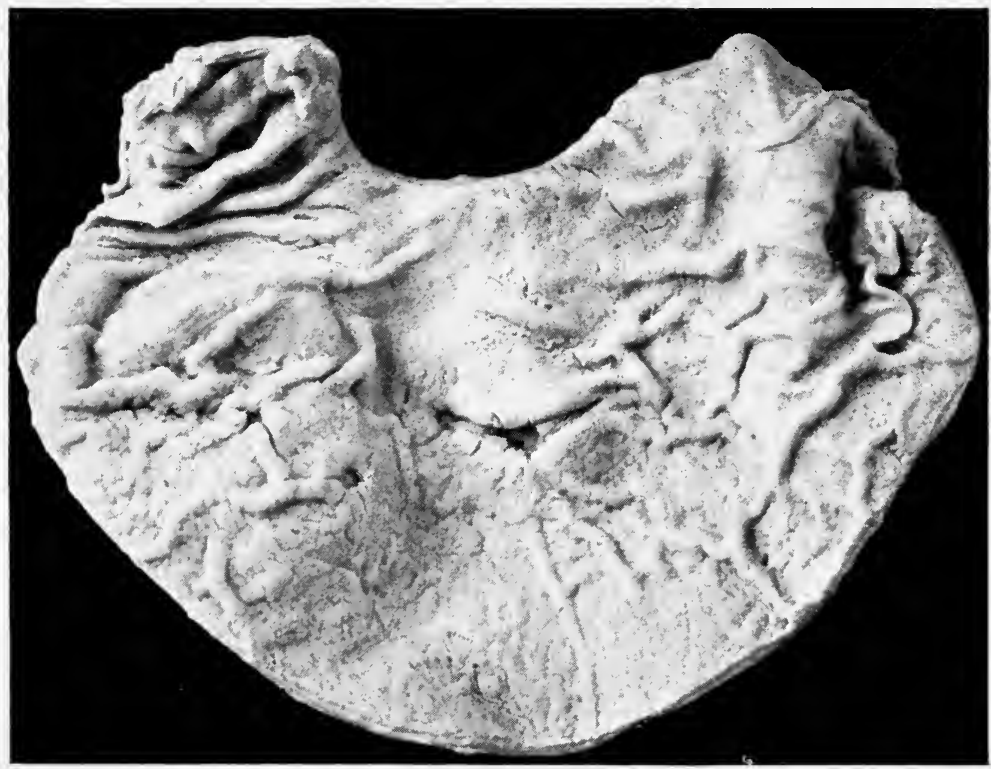

FIG. 54.-Perforation of a gastric ulcer in an infant. (Porter Parkinson.) 
It is noteworthy that of the perforations in the anterior wall only three were near the greater curvature; all the others were situated in the upper half of the anterior wall, and mostly near the lesser curvature. The site of perforation differs in the two sexes; in the male the area of perforation is near the pyloric end of the stomach, while in the female perforation occurs more often towards the cardiac end of this viscus.

A second ulcer was present in 23 per cent. of this series of cases. The size of the perforation varies from a mere pinprick to the size of half a crown, the average being about the size of a threepenny piece.

The suddenness of the onset, and the severity of the symptoms, depend probably in a very large measure on the size of the aperture, and the quality and quantity of the gastric contents. In one of the most acute cases I have seen, perforation occurred two hours after a meal of sausages and potatoes. Although I operated within six hours, the whole abdomen was filled with a purulent fluid which gave cultures of streptococci.

The Previous History.-It is a common belief that a considerable number of the patients who are the victims of perforation, have not suffered previously from indigestion. According to some writers the proportion of such cases is as high as 64 per cent. This is not in accord with my experience. In the great majority of cases, a history of previous gastric trouble can be obtained, if careful inquiry be made. I have met with only one instance in which, on cross-examination, no indications of previous gastric trouble could be elicited.

In the majority of cases the onset of perforation is sudden and unexpected, but in some (about 17 per cent.) there are premonitory symptoms, in the form of increased epigastric pain, with or without vomiting. 
The Signs and Symptoms of Perforation.-In the literature of the subject there is perhaps no more vivid picture of gastric perforation than the historical account of the case of Henrietta, Duchess of Orleans, which has been handed down to us by one of her maids-in-waiting. This interesting story is as follows :

"She had ailed for some time from pain in the stomach and side, but seemed to be otherwise in good health. One day after dinner she complained several times of pain. She drank some succory water as a stomachic, and, putting the cup down with one hand, with the other she pressed her side, and said in a voice which betokened much suffering: 'Ha! what a stitch in the side; what pain! I cannot bear it.' Speaking these words, she flushed, and a moment afterwards turned pale with a wan lividity which astonished everybody. She kept crying out, and begged to be carried away, for she could not longer hold up. Supported by the arms of others, she managed to walk, but with difficulty, and bent double. Put to bed, she cried out more than ever that the pain in the pit of her stomach was past belief. She turned from side to side. A physician was fetched, who pronounced her complaint to be colic, and who prescribed suitable remedies. But the pain continued. She said that her suffering was greater than could be conceived, and that she would die.

"All this occurred in less than half an hour. Whatever she swallowed made her retch; she brought up only a little mucus mixed with food. The efforts to vomit and the excessive pain threw her into a state of exhaustion which resembled repose, but she told the bystanders not to deceive themselves; that the pain was as great as ever, and that she had no strength left to cry out. She heard someone remark that she was easier, and she said: "That is so far from being true that were I not a Christian I would kill myself, so great are my sufferings. It is wrong to wish evil to anyone,' she added, 'yet I would that somebody could feel for a moment what I feel, so as to know what my 
pain is like.' Her pulse became imperceptible, her limbs cold. Her friends anxiously asked if nothing more could be done; they suggested a score of remedies, and at last her physicians in sheer desperation made an attempt to bleed her, but the blood would not flow. They gave her some broth, for she had taken no food since dinner. She no sooner swallowed it than her sufferings increased. She complained that her stomach was filling up. Death was painted on her face. The last struggle was short, and after two or three convulsive movements about her mouth, she died nine hours from the beginning of her illness."

Suddenness of onset is the most characteristic feature of acute perforation. Suddenly the patient is seized with intense abdominal pain. In some instances, at the time of the seizure, the patient experiences a feeling as of something giving way. At first the pain is limited to the epigastrium, but soon it becomes general, and is referred to the whole of the abdomen. It is so acute and agonising as to be almost unbearable, and the patient's face bears witness to the severity of his sufferings. The extremities are cold, beads of perspiration appear on the forehead, the respirations are quick and shallow, and the patient replies to questions in short, jerky sentences. The body temperature is subnormal, often as low as $95^{\circ} \mathrm{F}$. or $96^{\circ} \mathrm{F}$. At first the pulserate is quickened, and its volume poor, but if the patient be not seen until the first shock of the seizure has passed off, the pulse-rate may be quickened little, if at all, and the volume good. The abdomen is retracted, and there is a " board-like rigidity"-a sign most significant of perforation of a hollow viscus. This rigidity is most marked over the upper part of the recti muscles, and sometimes is limited to this region. Usually there is tenderness of the upper part of the abdomen; the gentlest pressure induces protective spasm of the abdominal muscles at once. 
As a rule, the point of maximum tenderness corresponds to the site of perforation. Absence of liver dullness, as determined by percussion, is a sign of no practical importance. Authorities differ as to the frequency of vomiting. In my experience it is present in about half the cases. Such are the early symptoms of perforation. In rare instances the inaugural shock is so profound as to prove fatal immediately (Maurice, Moynihan).

Often the symptoms of collapse pass off, the pulse improves, increasing in volume and decreasing in frequency, and the temperature may regain the normal. The pain continues, less agonising maybe, but no less constant. The improvement, however, is short-lived. Gradually but surely, fresh symptoms appear, harbingers of peritonitis caused by the presence of gastric contents in the peritoneal cavity. First and most important, there is a steady and progressive increase in the pulse-rate, gradually too its volume diminishes until it becomes the small, rapid, running pulse indicative of advanced septic peritonitis. The temperature may rise to $100^{\circ} \mathrm{F}$. or $101^{\circ} \mathrm{F}$; ; the retraction of the abdomen is succeeded by gradually increasing distension; the abdomen becomes tense and tympanitic; the respiration more and more thoracic, the extremities cold, the face pinched and cyanosed, and the patient slowly sinks, life rarely being prolonged more than a few days.

The following is an illustrative case :

A woman, aged thirty-five years, had suffered from epigastric pain for two years. At first the pain used to come on from half to one hour after food, but latterly it had had no definite relation to food, but had been worse at night. Sometimes the pain was relieved by food, but it was relieved always by vomiting. The vomit was very 'acid' and frothy, and dark in colour. One morning at 8 A.M. she was seized suddenly with severe abdominal pain and vomiting. Three and a half hours later she was ad- 
mitted into the hospital. On admission she was extremely collapsed, cold and blanched, and apparently in great pain. Her temperature was $97^{\circ} \mathrm{F}$., pulse-rate 140 , small and feeble. Immediately after admission she vomited two pints of material containing some blood. Her abdomen was distended, with marked rigidity and tenderness in the upper part, especially to the left of the middle line. While preparations for operation were in progress, a pint of saline was run into the rectum. Open ether was administered, and I opened the abdomen. There was much turbid fluid free in the peritoneal cavity. The stomach was very large, and was divided into two compartments by an hour-glass constriction at the junction of the pyloric with the middle third. Immediately to the distal side of the constriction, close to the lesser curvature, there was a perforated ulcer, the size of a sixpenny piece. The perforation was sewn up with two rows of sutures. Anterior gastro-jejunostomy was performed on the proximal side of the constriction, a large drainage tube was placed in Douglas' pouch and brought out through a stab incision above the pubes, and the wound was sewn up. The operation lasted twenty-two minutes. During the operation, and again while being carried back to bed, the patient stopped breathing, but recovered upon artificial respiration being performed. After operation the patient was in a condition of extreme collapse, very cold, pulse 140 , almost imperceptible. Saline was given by the axillæ and by the rectum, and thanks to the devoted nursing of the Sister-in-charge she rallied gradually and made an uneventful recovery. A year later she had gained over three stone in weight, and wrote, "I don't remember ever feeling so well as I do at the present time. I can eat almost anything."

The Diagnosis of Perforation.-When signs of general peritonitis are present, the diagnosis presents no difficulty. It should be our aim, however, to make a diagnosis and to act upon it at a much earlier stage, but this is not always easy, as is shown by the mistakes made by surgeons of great experience. 
In a few cases primary collapse is succeeded by a period of reaction, so that when the patient is seen his general condition may be such as to lead to doubt as to the existence of so serious a lesion as perforation of an ulcer. The pulse-rate may be not more than 80 , its volume good, the temperature not raised, and beyond a little tenderness, nothing is discovered on examination of the abdomen. It is of the utmost importance to realise and to remember, that a normal pulse and temperature do not negative the existence of perforation. Decision in such cases is beset with difficulty. There is little but the history to guide us, and if we rely on this, we may perform an unnecessary operation; on the other hand, if we delay, the lull may be succeeded by a storm which tells us, unmistakably, that intervention is too late.

Difficulty in diagnosis is likely to arise especially in cases in which morphia has been administered. In dealing with cases of supposed perforation, it is a golden rule, never to give morphia except under one of two conditions : first, when immediate operation is decided on; secondly, when a diagnosis of perforation has been excluded absolutely. As soon as it is decided to operate, the morphia and atropine, always injected before the administration of ether, should be given at once. The patient is relieved of pain, and probably his general condition will have improved considerably by the time the preparations for the operation have been completed.

Although from time to time difficult cases are met with, in the vast majority it is possible, with care, to make a diagnosis of perforation. The important points are :

(i) A history of previous indigestion.

(ii) The sudden onset of agonising pain.

(iii) Rigidity of the upper part of the recti muscles. In the rare instances in which rigidity is absent, careful in- 
spection always will reveal impaired mobility of the upper part of the abdomen.

Differential Diagnosis. - The chief diseases which give rise to difficulty in the diagnosis are: early pneumonia or diaphragmatic pleurisy, acute ptomaine poisoning, acute appendicitis, bilary colic, ruptured tubal gestation, and acute pancreatitis.

Of these, the first two are by far the most important, not only because sometimes the differential diagnosis is very difficult, but because in these diseases an operation is absolutely unnecessary and harmful.

In acute appendicitis, ruptured tubal gestation, and in acute biliary colic, surgical treatment is indicated, and so an exact diagnosis is of less importance.

In Early Pneumonia or Diaphragmatic Pleurisy, the most important diagnostic point is the marked increase in the rate of respiration in relation to the pulse-rate. A respiratory rate of thirty-four or more to the minute with a pulserate of a hundred or less, suggests a thoracic and not an abdominal lesion. In pneumonia or pleurisy there is impaired movement of the lower part of the chest, and the abdominal tenderness and rigidity is far less marked than in perforation. The rigidity is not continuous, the muscles may be induced to relax by gradual, firm pressure with the hand, or by instructing the patient to take a slow, deep expiration.

Acute Ptomaine Poisoning may simulate perforation so closely, that at times it is well-nigh impossible to distinguish between the conditions. The points that may be of service are: the possible history of others being attacked in the same way, the occurrence of diarrhœa, and the character of the vomit, which, in ptomaine poisoning, consists usually of undigested food, without that copious secretion of gastric juice and bile which characterises the vomiting, when present, in perforation. 
A woman, aged twenty-six years, was seized, while out walking, with severe abdominal pain, and fell down in a state of collapse; her extremities were cold, temperature $97 \cdot 4^{\circ} \mathrm{F}$., pulse 80 , respirations 28 . She was well enough to give a clear account of herself, and it was ascertained that she had been in hospital three times for attacks of abdominal pain.

There was some rigidity of both recti above the umbilicus, and apparently pain on palpating the upper part of the abdomen. She vomited slightly after admission. A few hours later her pulse became poor and irregular, and it was thought that she was suffering from a perforated gastric ulcer.

I opened the abdomen, but found nothing to account for her severe symptoms. After she had recovered, we discovered that she was much addicted to drink, and apparently her symptoms were the result of a drinking bout.

In Acute Appendicitis, the rise of temperature, coincident with the initial onset of pain, usually will distinguish this affection from perforation of an ulcer, in which there is at first a fall of temperature. It is important to ascertain whether the pain began in the epigastrium, or in the appendix region.

Some years ago I was asked to see a young man who was seized suddenly with severe abdominal pain. His temperature was subnormal, pulse 96 , and there was extreme tenderness and rigidity over the epigastrium. A diagnosis of perforated gastric ulcer was made, but a small incision above the umbilicus showed that there was no gas or extravasated fluid in the peritoneal cavity. Some months later the patient had a somewhat similar attack, but on this occasion there was extreme tenderness over the appendix. After the acute attack had subsided the abdomen was reopened and a diseased appendix removed. He had evidently had previous attacks of appendicitis.

I have little doubt that when I operated on him, his 
symptoms were really due to acute appendicitis. The mimicry of gastric perforation was remarkable, and I think in such a case a correct diagnosis is impossible.

It is well known that chronic appendicitis may mimic gastric ulcer closely, but this is the only instance ${ }^{1}$ with which I am acquainted in which an acute attack of appendicitis has simulated perforation of a gastric ulcer.

In Biliary Colic the pain may be of agonising intensity, but it is rarely accompanied by such severe initial collapse. Usually the temperature is normal, but there may be pyrexia. Hiccough is commonly a distressing symptom, and vomiting is frequent. The attack is often ushered in by shivering or by a rigor. The pain is referred to the right hypochondrium, and often there is radiation to the right scapular region. In cholelithiasis there may be a history of discomfort and flatulence after food, but not the definite and regular relation of pain to the ingestion of food which obtains in ulcer. In biliary colic there is a history of previous attacks, some of which may have been accompanied by jaundice.

In Ruptured Tubal Gestation the history of previous amenorrhœa will give a clue to the correct interpretation of the symptoms, but it must be borne in mind that a history of vomiting of pregnancy may suggest gastric ulcer. In ruptured tubal gestation, rigidity is more marked in the lower part of the abdomen, and it rarely is so pronounced as in perforation of an ulcer. In tubal gestation the maximum tenderness is below, in perforation above, the umbilicus. As in both these conditions surgical treatment is required, exact differentiation is not essential previous to operation. On opening the abdomen, the presence of blood in the peritoneal cavity points at once to the presence of a ruptured tubal gestation.

1 I have operated since in a case of acute appendicitis, in which a severe attack of hæmatemesis suggested at first a diagnosis of gastric ulcer. 
In Acute Pancreatitis the pain is severe, and is referred to the epigastrium, but as a rule there have been no previous symptoms suggesting an ulcer. From the first the pulserate is very rapid, vomiting is frequent, and the general condition of the patient very alarming considering the short duration of the illness.

In cases of doubt I hold very strongly that it is better to open the abdomen than to delay, provided that the surgeon definitely make up his mind to limit his operation to the making of a simple incision in order to determine whether there be free fluid, or gas in the peritoneal cavity. If the surgeon be content with this, he will do no harm. The danger is that he may proceed to search for some other seat of mischief, and instead of being a simple procedure, unattended by serious risk, the operation may be so prolonged as to jeopardise the patient's life. In cases of doubt there must be no indecision. The surgeon must resolve firmly, to make only a small incision, and to close the abdomen immediately, if there be no free gas and no extravasated fluid in the peritoneal cavity. On no account should the operator allow himself to begin an extensive search for some other condition, unless the indications be very definite.

\section{B. Perforation of Duodenal Ulcer}

Perforation of an Acute Duodenal Ulcer is an occasional occurrence. Acute duodenal ulcers usually are of infective or toxic origin, and are associated with extensive cutaneous burns, or with acute appendicitis. I have had one case in which an acute duodenal ulcer perforated a few days after operation for acute appendicitis.

As a rule, patients with acute duodenal ulcer are so ill, that any operation is out of the question, even if the existence of a perforation be recognised. 
Perforation of a Chronic Duodenal Ulcer is found usually on the anterior wall of the duodenum within an inch and a half of the pylorus. I have met with one instance of perforation of an ulcer on the posterior wall of the second portion of the duodenum, a position very difficult of access.

Perforation of a duodenal ulcer is a more serious catastrophe even than perforation of a gastric ulcer. In a few cases of perforated gastric ulcer, the aperture becomes closed by a plug of lymph or omentum. Rare as is such a happy issue in perforated gastric ulcer, it is more rare after perforation of a duodenal ulcer.

The clinical picture of perforated duodenal ulcer is very similar to that of perforated gastric ulcer. There is the same sudden onset of agonising pain, a similar board-like rigidity of the abdominal walls, a similar progressive sequence of untoward symptoms, the same urgent indication for surgical treatment, and a like fatal issue if no operation be performed. Little, therefore, need be added to what has been written already in the previous part of this chapter.

Perforation of a duodenal ulcer has to be distinguished from the same acute conditions as in the case of perforated gastric ulcer. As a rule, the differential diagnosis between a perforated gastric and duodenal ulcer is not difficult. The history of pain coming on two or more hours after food, the periodicity of the attacks, and the intervals of good health, point to the duodenum as the site of the ulcer. After perforation, the point of maximum tenderness usually indicates the site of the ulcer.

In perforation of duodenal ulcer, the mimicry of acute appendicitis may be most marked and perplexing, and frequently it has led to mistakes. The reason of this mimicry is, that in perforated duodenal ulcer the extravasated fluid gravitates to the right iliac fossa, and causes pain, rigidity, and tenderness in this region. To add to the difficulty in 
diagnosis, in some cases of appendicitis there is a history of pain after food. As a rule, however, the interval between the taking of food and the onset of pain is much shorter than is the case in duodenal ulcer. In appendicitis it is rare to get such a clear-cut picture of pain recurring in so definite and ordered a manner as obtains in duodenal ulcer. In both conditions there is tenderness in the right iliac fossa, but in acute appendicitis the rigidity is less marked, and the pain far less agonising than in perforated duodenal ulcer. In acute appendicitis there may be tenderness in the right hypochondrium, but much less marked than is the case in duodenal perforation.

Now that we recognise and are prepared for this pitfall, errors in diagnosis are far less common. In the great majority of cases a correct diagnosis may be made by attention to the following points :

1. The history.

2. The point of maximum tenderness.

3. The temperature-an initial rise in acute appendicitis, and, as a rule, an initial fall in acute perforation of an ulcer.

4. In my experience, in cases of perforated duodenal ulcer, often there is dullness in the right flank, which disappears in part when the patient is turned on his left side. This sign, present on the right but not on the left, is very suggestive of perforated duodenal ulcer.

The Treatment of Acute Perforation of Gastric or Duodenal Ulcers.-There is only one method of treatment for acute perforation, and that is immediate operation. There are on record undoubted instances of recovery without operation. The following case is probably an instance :

An elderly lady, who had suffered from indigestion for years, was sent to me by Dr. Soltau Fenwick. Nine years previously, on the way to Paris, she was seized suddenly with severe 
abdominal pain, followed by signs of general peritonitis. She was laid up for many weeks, but eventually recovered. At the operation (nine years later) I had great difficulty in finding the peritoneal cavity owing to extensive adhesions. The upper two feet of the jejunum were matted together by dense adhesions, evidently the result of an old perforation. After a tedious dissection I succeeded in separating the adhesions, and performed gastro-jejunostomy. The patient made an excellent recovery.

Such a favourable result is extremely rare, and is no argument against the rule of immediate operation. No case is too bad for operation, and there must be no waiting for a quiescent period which may never come. Whilst preparations for the operation are in progress, a pint or so of saline should be administered by the rectum, and half a pint in each axilla. By these means, and by the application of plenty of warmth, preferably in the form of radiant heat from electric lamps suspended from a cradle, even a most desperate case may be tided safely through the operation, provided that it is performed with rapidity and gentleness. With regard to the anæsthetic, this should be either gas and oxygen, or ether. I hold very strongly that in these cases, as indeed in all acute abdominal cases, the use of chloroform is absolutely contra-indicated, and prejudices greatly the patient's chance of recovery.

The Technique of Operation for Acute Perforation of Gastric and Duodenal Ulcers.-An incision $1 \frac{1}{2}$ inches long is made in the middle line above the umbilicus. If gas or free fluid be found in the peritoneal cavity, the incision is enlarged to three inches. If gastric contents are found distributed generally throughout the peritoneal cavity, I make a stab incision above the pubes at once, through which I pass a large Keith's tube to the bottom of the pelvic cavity. During the remainder of the operation a nurse is instructed 
to suck out, at intervals, the fluid in the abdomen by means of a glass syringe attached to a rubber tube of suitable size for being passed into the Keith's tube.

Then a rapid search is made for the perforation. As a rule it will save time if, in a male patient, the duodenum be examined first, in a female patient, the stomach. Often the site of perforation is indicated by a thick deposit of lymph. The perforation is sewn up by two rows of sutures, the first penetrating all coats, the second row being serous sutures. If there be much induration round the perforation, or if the surrounding tissues be very friable, suture of the perforation may be very difficult; but the surgeon must not be satisfied until, by some means, he has effected a complete closure. When complete closure by suture is impracticable, the aperture may be closed by stitching a piece of omentum or small intestine over it, or, as I have done on one occasion, by doubling over a piece of the stomach and stitching it round the edge of the perforation.

Invariably after closure of the perforation I perform a gastro-jejunostomy. I advocated this as a routine measure in my Hunterian lectures delivered in 1906. Briefly my reasons are as follows: As I shall point out later, in dealing with the remote results of operation for perforation, 23 per cent. of the patients who recover from operation relapse subsequently. This in itself is an argument in favour of my practice. But some more urgent reason is required. Our concern is with the immediate saving of life, the ultimate result is a secondary consideration. My contention is that the routine performance of gastro-jejunostomy diminishes the mortality following operation for perforated ulcer. I will indicate briefly the grounds of this belief.

A perusal of hospital records shows clearly that a number of patients die shortly after operation for perforation, either from perforation of a second ulcer, from hæmatemesis, or 
from leakage along the line of suture. In a series of 112 consecutive cases $I$ found that 11 per cent. of the patients died from one or other of these accidents. In a series of cases published by Dr. H. P. Hawkins, the mortality rate from these causes was 12 per cent. It will scarcely be denied that the danger of perforation of a second ulcer and the risk of hæmorrhage are diminished greatly, if a gastrojejunostomy be performed. Further, if the ulcer be situated near the pylorus, often it is impossible to invaginate the ulcer thoroughly without causing narrowing of the pyloric orifice. In his anxiety to avoid this, the surgeon is apt to turn in a margin round the ulcer insufficient to secure firm union, with the result that the sutures cut out, and allow fresh extravasation of gastric contents. If, however, a gastro-jejunostomy be performed, the ulcer may be enfolded as freely as may seem desirable; and further, owing to the altered physiological conditions produced by the short circuit, far better conditions are secured to promote healing of the line of suture.

I think, therefore, that we are justified in regarding as preventable the deaths which are due to the causes indicated above; and the presumption is strong, that a considerable proportion of these deaths would be prevented by the performance of gastro-jejunostomy. I do not claim a certainty, only a possibility, but I do urge that a procedure which holds out even a possibility of reducing the mortality from perforation by 11 per cent. deserves a very extended trial.

There is, however, an even more weighty reason than any of the foregoing. There can be little doubt that the most effective means of draining the peritoneal cavity is through the intestines and through the intestinal lymphatics. In order to promote and encourage this drainage, our aim should be to start intestinal movements as soon as possible after operation. After gastro-jejunostomy, purgatives may 
be given at once with absolute safety, and from experience I am convinced that they act much more certainly and much more quickly when there is a short circuit between the stomach and the jejunum.

This suggestion has been much criticised, but more extended experience only has confirmed me in my belief that it is right, and that the performance of gastro-jejunostomy in all cases, not only improves greatly the patient's immediate chance of recovery, but ensures a more complete and permanent cure.

Two objections have been raised to this procedure : first, that the tissues are not in a condition for a plastic operation, and secondly, that it prolongs the operation unduly.

In my opinion neither objection is valid. My experience is, that even in cases of septic peritonitis, peritoneal surfaces unite perfectly well, if kept in apposition by the firm pressure of a continuous suture. To the objection that the performance of gastro-jejunostomy prolongs the operation unduly, my answer is, that it ought not to prolong the operation more than five, or at the outside, seven minutes. Several times I have sutured a perforated gastric ulcer and performed gastro-jejunostomy in less than twenty-five minutes.

The illustrative case of perforated gastric ulcer which is related earlier in this chapter (p. 158), is a good example of the value of gastro-jejunostomy; without it I think recovery would have been impossible.

A well-known writer, commenting on my advocacy of a primary gastro-jejunostomy, states that in some very early cases a gastro-jejunostomy may be performed primarily, but, that in the majority of cases, it is safer to perform it at a second operation. He has missed the point of my argument. My chief reason for advocating a primary gastro-jejunostomy is, not to guard against future recurrence, but to improve the patient's immediate chance of recovery. It is in the later, 
and not in the earlier cases, that gastro-jejunostomy is indicated most emphatically.

To recapitulate, the performance of gastro-jejunostomy in addition to suture of a perforated ulcer:

1. Lessens the chance of perforation of a second ulcer in cases in which more than one ulcer is present.

2. Diminishes the risk of a fatal issue in cases in which there happens to be two perforated ulcers, only one of which is discovered.

3. Minimises the risk of hæmatemesis from the ulcer after operation.

4. Allows more efficient enfolding of the ulcer in cases in which the ulcer is in the duodenum or in the pyloric third of the stomach.

5. Promotes more rapid and certain healing of the line of suture, and so lessens the risk of further leakage.

6. Permits earlier feeding, earlier administration and more rapid action of purgatives, and so secures more efficient drainage of the peritoneal cavity.

7. Lastly, but of less importance, it improves the remote result in those patients who recover.

As regards the toilet of the peritoneum, I hold very strongly that this is best left to Nature. My belief is that flushing simply increases shock, and is ineffectual in cleansing the peritoneal cavity. Neither do I employ mopping or sponging, as I think it is much more important to get the patient back to bed as quickly as possible. When once the perforation has been closed, the peritoneum can deal with extravasated fluid much better than can the surgeon. The key to the situation is the promotion of drainage through the intestinal lymphatics by early intestinal peristalsis.

There is considerable difference of opinion as to the advisability of drainage. My own view is this : I believe that drainage does no harm; I am convinced that in some 
cases it does good. Acting, therefore, on the principle, never to take risks which can be avoided, my inclination is in favour of this precaution, neglect of which may do harm, the adoption of which has no injurious result, and may prove of benefit. My usual practice is to put a tube down to the bottom of the pelvis through a stab incision above the pubes, and occasionally in late cases, I leave a drain in the region of the perforation.

As a general rule, at the conclusion of the operation it is advisable to pass a tube down the osophagus and to wash out the stomach.

Rapidity and gentleness are the main secret of success in these operations. The whole operation, including the performance of gastro-jejunostomy, should be completed in twenty-five; or at the outside, in thirty minutes.

After-treatment.-The patient should be propped up in bed in a sitting position. If there be much shock an injection of pituitary extract ( 1 c.c. $=17$ minims) is administered intramusculary. In all cases continuous saline is given by the rectum for at least forty-eight hours. I never give morphia or strychnine. With a view to draining the intestines and the intestinal lymphatics, I commence the administration of calomel early in these cases. If, at the time of the operation, there be general peritonitis, or an extensive extravasation of gastric contents, the first dose is given as soon as the patient regains consciousness. In less serious cases, the administration of calomel is delayed until the evening or the following morning.

The calomel is prescribed in half-grain doses, repeated every hour or two hours, until three grains or more have been taken. Two hours after the last dose, a seidlitz powder is given, followed an hour or so later by an enema, if necessary. The promotion of early intestinal peristalsis is of the utmost importance, so much so that I am accustomed to 
regard the patient as well on the way to recovery, when once there has been a satisfactory evacuation of the bowels.

Feeding is regulated by the condition of the patient. If the patient be in fair condition, and there be much fluid in the peritoneal cavity, nothing is given by the mouth until the bowels have been opened. If, on the other hand, there be little extravasation of gastric contents, or the patient be in bad condition, or very emaciated, I commence the feeding at once, with diluted milk (milk one part, water two parts). In weak, elderly, or feeble patients, usually I give an ounce or two of Sanatogen in milk during the course of twentyfour hours. After the bowels have been opened, soft solids and fluids ad libitum, are allowed. When a drainage tube has been used, usually it is left in for a week.

Vomiting is rare after operation, but if it occur the surgeon must be on the watch for acute dilatation of the stomach. If this occur, the stomach should be washed out, and the administration of calomel repeated, followed, if necessary, by a seidlitz powder.

Immediate Results.-Although the results obtained by the surgical treatment of perforation are better than formerly, they are still far from ideal. In 1898 Dr. Keen prophesied that the time was approaching when operations for perforation would be performed within twelve hours, and the mortality would be reduced to 10 per cent. This prediction still awaits fulfilment. In the year 1904 the mortality rate of the cases operated on in twelve of the London hospitals is 48 per cent. Through the courtesy of the surgical registrars of fourteen of the London hospitals, I have been able to ascertain that during the year 1910, 143 operations were performed for perforated gastric ulcer : of these patients 64 died, a mortality rate of 44 per cent. For perforated duodenal ulcer 58 operations were performed : of these patients 23 died, a mortality rate of 39 per cent. 
Thus so recently as the year 1910 the percentage of deaths following operation for perforated gastric and duodenal ulcers was still as high as 43 per cent.

Many writers have laid stress on the importance of early operation. The tables published by Weir and Foote, and Keen and Tinker, have been quoted extensively as showing that the cases of early operation have a much higher percentage of recoveries than have those operated on later. Thus in Weir and Foote's series the mortality of those cases operated on within twelve hours is 39 per cent., and 76 per cent. in operations performed between twelve and twentyfour hours after perforation. In Keen and Tinker's tables of seventy-eight cases, the mortality of those operated on within twelve hours is 19 per cent., and of those operated on within from twelve to twenty-four hours 50 per cent. Keen and Tinker also divide the cases in their table into two periods, those before the year 1896 and those after that year. In the first period the mortality of those operated on within twelve hours is 28.5 per cent., in the second period it is only 16.6 per cent. "This rapid fall," remarks Dr. Keen, "in the mortality of the cases operated on within the same interval after perforation shows that our technique, and therefore our results, are improving."

These tables, however, do not justify this sanguine conclusion, for they are not consecutive, but isolated, published cases, and therefore comprise an undue proportion of successes, for of the 78 cases, 52-that is, 66 per cent.-of the patients recovered. In the series of consecutive cases referred to above (p. 154), the mortality rate, even in those operated on within twelve hours, is 47 per cent.; in those operated on within from twelve to twenty-four hours, 53 per cent.; in those operated on within from twenty-four to forty-eight hours, 83 per cent. ; and in those operated on over fortyeight hours after perforation, 50 per cent. So that in the 
consecutive series of cases, early operation does not seem to have quite the importance which Keen and Tinker's table appears to show. I need hardly say, I do not suggest that operation should be delayed. Undoubtedly, the earlier operation is performed, the better. The figures which I have given, show the untrustworthiness of statistics compiled from isolated cases, and also that other factors besides early operation may have an important bearing on the result, and of these probably the amount and nature of the extravasation, the presence of other ulcers, and the rapidity and completeness with which the operation is performed, are the most important. But while admitting this, we must not lose sight of the axiom, that perforated gastric or duodenal ulcer is a catastrophe demanding early recognition and immediate operation.

Remote Results.-In view of the suggested advisability of performing gastro-jejunostomy it is important to ascertain what proportion of the patients operated on for perforations suffer subsequently from gastric troubles. Numerous isolated instances of relapse have been recorded.

This subject is one which merits full investigation by inquiry into the after-history of a large number of patients in whom perforated ulcers have been sutured. Of 54 patients in my series who recovered after operation for perforated gastric or duodenal ulcer, I have traced 32. All those in whom gastro-jejunostomy was performed in addition to suture of the ulcer, have remained quite well, with the exception of one patient who died from spinal meningitis. Of the remainder, 17 have suffered from gastric trouble. Of these 17 patients, one died from a second perforation, two subsequently required gastro-jejunostomy, while at the time of inquiry nine had symptoms suggestive of ulcer, and five had symptoms due probably to simple dyspepsia. Therefore, if we regard these figures in the most favourable light, 
by assuming that all the untraced patients have remained quite well, we see that of the cases in which gastro-jejunostomy was not performed 12 patients-that is, nearly 23 per cent. of the patients who recovered-have had symptoms suggesting a recurrence of ulcer, one case proving fatal, and two patients requiring further operation. The ultimate results in the cases comprising the series published by Dr. Hawkins, to which I have alluded, are almost identical. No less than 23 per cent. of the patients sought relief at the hospital for gastric trouble after their operation.

A study, therefore, of the after-histories of patients operated on for perforated gastric or duodenal ulcer, appears to me to furnish most convincing evidence in favour of my contention, that gastro-jejunostomy as well as suture of the ulcer, is indicated in the treatment of perforated gastric and duodenal ulcers.

Chronic Perforation.-In chronic perforation the ulcer destroys the gastric wall slowly, while plastic peritonitis at the base of the ulcer results simultaneously in adhesion of the stomach to surrounding parts. Thus, when the base of the ulcer is destroyed, the escape of the gastric contents is limited by the adhesions which have been formed. Chronic perforation of a gastric ulcer is more common in the posterior wall of the stomach, and frequently the ulcer erodes the pancreas. If the adhesions yield, gastric contents may leak slowly into the surrounding adhesions, leading to a perigastric abscess. The abscess thus formed may increase slowly in size, and finally rupture into a hollow viscus, or track upwards (sub-phrenic-abscess) and perforate the diaphragm into the pleural or the pericardial cavity. A sub-phrenic abscess may be anterior or posterior. An anterior abscess results from perforation of an ulcer of the anterior gastric wall. The pus tracks upwards between the right lobe of the liver and the diaphragm to the right of the falciform ligament, 
or between the left lobe and the diaphragm to the left of the falciform ligament. In some instances the abscess may destroy the overlying skin by pressure, and so discharge externally, but more usually perforation of the diaphragm ensues. The lower limit of the abscess is formed by dense adhesions, the result of plastic peritonitis.

A posterior abscess is limited, at first by the boundaries of the lesser sac, and extends up to the diaphragm over the posterior and upper surfaces of the liver, while later, as in the case of an anterior abscess, perforation of the diaphragm may occur. It must be borne in mind, that a sub-phrenic abscess may result from imperfect drainage of the peritoneal cavity after operation for acute perforation.

Sub-phrenic abscessfromperforation of the stomach, or duodenum is far more common on the left side than on the right.

Symptoms and Diagnosis of Perigastric Abscess.-The symptoms of perigastric abscess may be very indefinite, the signs obscure, and consequently the diagnosis of some difficulty. In some cases there is a history of a sudden onset of pain, indicating the time at which perforation occurred. In others this suggestive feature is absent. The chief symptoms are those of septic absorption, daily rise of temperature, night sweats, occasional vomiting, and later, attacks of coughing accompanied by dyspnœa. As the abscess increases in size there may be physical signs of its presence-impaired movements and bulging of the lower part of the chest, and displacement of the heart. The sudden rupture of the abscess into a hollow viscus, or through the diaphragm into the pleura, may be attended with severe collapse.

The following case illustrates these features very well :

A man, aged twenty-six, suffering from a gastric ulcer, while asleep in hospital, was seized suddenly, during the night, with 
severe abdominal pain. A few hours later $I$ opened the abdomen and found a very extensive perforated gastric ulcer of the anterior wall of the stomach at the extreme cardiac end. I sutured the perforation and performed gastro-jejunostomy. The patient was desperately ill; a large area round the abdominal wound sloughed, exposing the intestines.

The temperature remained fairly regular until three weeks after the operation, when he had a sudden rise to $103^{\circ} \mathrm{F}$.

Gradually his temperature fell again, and became normal in a few days. A week later he had another sudden rise. During the next three weeks he had a hectic temperature varying between $98^{\circ} \mathrm{F}$. and $103.8^{\circ} \mathrm{F}$., with occasional vomiting. A sub-phrenic abscess was suspected, and he was explored on three occasions, but no pus was discovered. A few days later he had a severe rigor, and after this the daily variations in temperature were less great. One week later he had a sudden attack of severe dyspnœa, accompanied by extreme collapse, and a pulserate of 140. The whole of the left side of the chest was dull to percussion. He was aspirated, and 63 ounces of pus were drawn off. After being desperately ill for a few days he improved slowly; the temperature varied between $98^{\circ} \mathrm{F}$. and $100^{\circ} \mathrm{F}$.

One month later he was aspirated again, and 40 ounces of pus withdrawn. Four days later a rib was resected, and a drainage tube inserted. Thereafter he improved steadily, and was discharged with a sinus at the site of resection, after having been seven months in hospital. The sinus persisted for nearly a year after his discharge, and I suggested that he should come in and have a further resection of ribs. The mere suggestion of an operation was sufficient, as within a month the sinus was soundly healed. Seven years later the man was in perfect health and suffered no inconvenience from weakness of the abdominal wall, although over an area of three inches above the umbilicus this consisted of skin only.

The Treatment of Sub-phrenic Abscess.-Sub-phrenic abscess is inevitably fatal, unless it be treated surgically. 
As a rule a sub-phrenic abscess is best opened and drained through the pleura after resection of one or more ribs. An incision about four inches long is made over the ninth rib on the right side, or over the seventh on the left side, and about three or four inches of rib resected. If the pleura do not contain fluid, the diaphragm and both layers of pleura are stitched to the chest wall. If the ribs be close together it may be necessary to resect portions of two ribs.

The edges of the wound are protected with gauze, and a director is pushed through the diaphragm into the abscess cavity. The opening is stretched with a pair of forceps and a large drainage tube inserted. It is not advisable to irrigate the abscess cavity either at the time of operation or subsequently. When a sub-phrenic abscess bulges in the epigastrium, or below the costal margin, it may be possible to open it by an incision in the epigastrium, or parallel with the costal margin. Sometimes a counter-opening in the loin may be made with advantage.

\section{REFERENCES}

Anderson, A. R., The Lancet, 1905, ii. p. 944. ।

Braun, Centralblatt f. Chir., 1897, p. 739.

Dowden, J. W., Edin. Med. Jour., Feb. 1909.

English, T. Crisp, Trans. Roy. Med. Chir. Soc., 1903, Ixxxvii. p. 27.

Finney, J. M. T., Annals of Surgery, July 1900.

Gould, Pearce, Brit. Med. Jour., 1894, ii. 861.

Hawkins, H. P., Med.-Chir. Trans., 1907, xc. p. 339.

Keen and Tinker, Philadelph. Med. Jour., 1898, p. 1104.

Kriege, Berliner Klin. Woch., Dec. 1892, p. 1280.

Maurice, The Lancet, 1894.

Mitchell, A. B., Brit. Med. Jour., 1905, ii. p. 779.

Moynihan, B. G. A., The Lancet, 1901, ii. p. 1656, and 1905, ii. p. 326.

Paterson, H. J., Gastric Surgery, 1906, p. 82.

Smith, Maynard, The Lancet, 1901, ii. p. 1658.

Weir and Foote, New York Med. News, April 25, 1896.

White, Sinclair, Brit. Med. Jour., Feb. 20, 1904. 


\section{CHAPTER XII}

\section{THE COMPLICATIONS OF GASTRIC AND DUODENAL ULCERS-continued}

\section{HÆMORRHAGE}

Gastrorrhagia or Gastric IHæmorrhage is made evident by hæmatemesis or melæna, or by both. It is a symptom both of acute and of chronic ulcer.

In a number of cases in which operation has been undertaken for gastric hæmorrhage, no definite ulcer has been found, but examination of the interior of the stomach has revealed a number of distinct and separate bleeding-points. More than thirty years ago Murchison reported two cases of fatal hæmorrhage from minute ulcers, which had perforated a small artery in the stomach wall. He observes that " they are remarkable for the minuteness of the ulcers, which are little more than hæmorrhagic erosions, but also for the absence of the usual symptoms of ulceration of the stomach. Neither of the patients suffered from vomiting prior to the occurrence of the hæmorrhage." Chiari, in 1882, described a similar case. More recently, Dieulafoy recorded a considerable number, and divided them into two groups-simple erosions and exulceratio simplex. The simple erosions appear to be mere abrasions of the gastric epithelium. In such cases the gastric mucous membrane, when examined during life, appears to be studded with minute bleedingpoints, probably the initial stage of the ordinary round ulcer of Cruveilhier. In exulceratio simplex there is a greater 
destruction of tissue, so that the minute arteries beneath the muscularis mucosæ are exposed.

Both varieties may give rise to alarming hæmorrhage. I have seen another variety which, so far as I know, has not been described. On opening a stomach subsequent to an extremely severe attack of hæmatemesis, a raised, vividly red patch, of the size of half a crown, was observed. No loss of substance was apparent, and the surface was oozing blood, seemingly from the acuteness of the inflammation. The wound was sewn up again and the patient had no further trouble, so that in the light of the after-history the operation was unnecessary.

Contrary to the accepted teaching, I believe that in many cases of duodenal ulcer, the hæmorrhage comes, not from the ulcer as is generally supposed, but from the mucous membrane of the stomach. In several of my cases of duodenal ulcer, microscopic sections of the gastric mucosa show blood escaping between the epithelial cells. Probably the same holds good in some cases of gastric ulcer.

Hæmatemesis cannot be regarded as pathognomonic of gastric ulcer. Not infrequently it occurs in cases of chronic appendicular disease, and is the effect of the hyper-secretion which, as I have shown elsewhere, often results from chronic appendicitis. Hæmorrhage from the stomach may also be secondary to cirrhosis of the liver, or due to rupture of varicose veins in the gastric mucosa, or to rupture of an aneurysm.

Since Dr. Soltau Fenwick pointed out the clinical and pathological distinction between acute and chronic ulcers, most clinicians are agreed that hæmatemesis due to an acute ulcer presents a totally different clinical picture from the hæmatemesis which results from a chronic gastric ulcer, and this difference has an important bearing on the surgical treatment of the bleeding. The pathology of the bleeding 
in acute ulcer and simple erosion is so similar, that surgically we need not draw any distinction between these two varieties.

In acute ulcer, according to Dr. Fenwick, severe hæmatemesis is the first indication of the disease in 75 per cent. of the cases. Usually the hæmorrhage is due to erosion of a small branch of the coronary artery, and is sudden and profuse, so that as much as a pint, or more may be lost in a few minutes. As a rule, Nature's means for arresting hæmorrhage, namely, the contraction of the artery, aided by the diminution of the heart's action due to loss of blood, are sufficient to stay the bleeding. The artery becomes occluded by the formation of an internal and external clot, and the hæmorrhage rarely is repeated.

In hæmorrhage from a chronic ulcer, on the other hand, there is almost invariably a history of chronic dyspepsia for months or years. The amount of bleeding varies from a minute quantity, only detected by an examination of the fæces for occult blood, to an amount so great as to lead to an immediately fatal issue (l'hémorrhagie foudroyante), although fortunately such an event is rare. As a rule the hæmorrhage is less copious and less alarming than that from an acute ulcer. There is also this important difference : the hæmorrhage from an acute ulcer once arrested is seldom repeated, the hæmorrhage from a chronic ulcer tends to recur after a quiescent interval, until the patient is reduced. to a condition of profound anæmia by repeated hæmorrhages. It is the repetition of the hæmorrhage from a chronic ulcer, which is so characteristic, and of such serious import.

What is the reason of this difference? In acute ulcer there is little or no surrounding induration, the arteries are not thickened by inflammatory infiltration, the tissues are soft and yielding, and consequently Nature is able to use her own means for the arrest of hæmorrhage.

In a chronic ulcer the eroded artery is thickened by 
chronic inflammation, and lies in rigid, indurated tissues, and so cannot close the opening in its wall by contraction. The formation of a clot is sufficient to arrest the bleeding temporarily, but the clot, exposed to the gastric juice, and to the discharge from the ulcer, becomes disintegrated, and leads to the recurrence of the hæmorrhage.

The Surgical Treatment of Gastrorrhagia.-The first suggestion of surgical intervention for gastric hæmorrhage was made by Rydygier in the year 1882. His suggestion, like many other original propositions, was received with coldness, and it was not until five years later that Mikulicz performed the first operation for hæmorrhage from a gastric ulcer, while it was reserved for Professor Roux of Lausanne, whose work I have had the privilege of witnessing, to have the credit of performing the first successful operation of this nature. In this case the source of bleeding was the coronary artery, and he excised the ulcer and ligatured the artery on both sides of the bleeding-point.

There is a considerable difference of opinion as to when to interfere in cases of gastric hæmorrhage. At present few are prepared to agree with Dieulafoy's opinion, that operation should be performed during the first bleeding, if as much as half a litre of blood be lost, or if, after a loss of a smaller quantity, the hæmorrhage be repeated within twenty-four hours. Dieulafoy believes that a third attack is likely to prove fatal, a view which, though not generally accepted, is supported in some measure by the facts recorded by Savariaud.

Granted, however, that surgical measures sometimes are indicated, the questions arise, In what class of case should we operate? When should we operate? and How should we operate?

1. In what Variety of Gastric Hæmorrhage is Operation indicated?-By recognising the distinction between bleeding 
from an acute ulcer or from a simple erosion, and bleeding from a chronic ulcer, we can form fairly clear indications for surgical treatment.

Pathologically, we may classify hæmorrhage from an acute ulcer as a primary hæmorrhage, and bleeding from chronic ulcer as a form of secondary hæmorrhage, and we can apply some of the principles which guide us in the treatment of primary and secondary hæmorrhage in other regions of the body. The first general principle in the treatment of primary hæmorrhage is, that no operation should be performed on an artery unless it bleeds. Therefore, in hæmorrhage from an acute ulcer, when once the bleeding has ceased, whether spontaneously or by the aid of medical art, we should hesitate to interfere. There is a reasonable expectation that the bleeding vessel will become securely occluded. If, in spite of absolute rest, the hæmorrhage start anew, we may conclude that Nature has failed, and if the second hæmorrhage has been a severe one, surgery must step in. But (and this is an important point) absolute rest must have been tried and have failed. I emphasize this, because in several of the cases I have seen, the second attack of hæmorrhage apparently has been recurrent or reactionary, due to displacement of the clot from too early stimulation, or injudicious moving of the patient. This also has been the case in some, at least, of the recorded cases. In these circumstances we may give Nature one more chance before deciding on operation. In bleeding from a chronic ulcer our guide must be the first general principle laid down for the treatment of secondary hæmorrhage-namely, that even if the bleeding have ceased, an operation is necessary to prevent its recurrence. Of course the degree of bleeding must be taken into account. Just as in slight secondary hæmorrhage elsewhere, we should first try simpler means; so an operation is not indicated necessarily after every slight hæmorrhage 
from a chronic ulcer. We must remember, however, that in chronic ulcer, it is probably not mechanical displacement, but septic disintegration, which starts the hæmorrhage afresh, so it is probable that the bleeding will be repeated.

Therefore, if already the patient have lost a considerable amount of blood, an operation is advisable to prevent the serious and possibly fatal result of further loss. Likewise, if the hæmorrhage, although slight, be frequently repeated, the indication for operation is clear. The risk of operation, if undertaken during a quiescent interval, probably is less than the risk from further hæmorrhage, and the patient gains a double advantage, prevention of further hæmorrhage and cure of the gastric ulcer.

2. When should we Operate?-Mikulicz took a very pessimistic view of the results of operation during the progress of acute hæmorrhage. He operated on four patients with only one recovery. Billroth had no better results. In the discussion on Mikulicz's paper, Korte gave similar testimony.

In spite of isolated successes, the weight of evidence seems against operation during the progress of the hæmorrhage ; the collapse induced by the loss of blood is favourable for the arrest of hæmorrhage, but unfavourable for operation, and there is not, as in perforation, the risk of increasing peritonitis through delay. Further, in by far the greater number of instances, it is not the initial hæmorrhage which causes death, but the repetition of it before the patient has recovered from the resulting anæmia. It is always dangerous to lay down hard and fast rules. To do so may give those who come after us cause " to reflect on the vanity of received opinion," but both from the cases I have seen, and from a study of the recorded cases, I think it is open to grave doubt, whether it is ever expedient to operate during the progress of gastric hæmorrhage. The number of cases in 
which the hæmorrhage is fatal immediately is very small ( 3 to 5 per cent.), and the percentage mortality of such cases is less than the mortality from operation during gastric hæmorrhage. There are quite a number of cases on record (and I have seen three myself) in which the stomach has been opened for acute hæmorrhage without the discovery of any definite source of bleeding. The stomach has been sewn up and the patient has recovered. Operation has thus been an unnecessary complication to the patient's convalescence. Such cases emphasize the advisability of trusting to Nature more, and resorting to surgery less, in the treatment of acute gastric hæmorrhage.

3. How should we Operate?-Many different procedures have been adopted. Mikulicz, in the four cases to which I have alluded already, applied the thermocautery to the base of the ulcer. Three of the patients died within twenty-four or forty-eight hours. The same method was employed by Korte, but the hæmorrhage continued until the death of the patient eight days later. In other cases the ulcer has been excised, ligatured en masse, or packed with iodoform gauze, and in others gastro-jejunostomy has been performed. In cases in which no definite ulcer has been found, the bleedingpoints have been picked up and ligatured en masse.

If we can rely on gastro-jejunostomy to prevent recurrence of the hæmorrhage, it is undoubtedly the simplest procedure to adopt, and we have good evidence that an efficient short circuit between the stomach and jejunum is an effective method of treating gastric hæmorrhage. Mr. Mansell Moullin advocates dealing with the bleeding-point, either by ligature en masse, or by excision of the ulcer. He argues in support of his view that there are at least two cases on record, in which bleeding has persisted in spite of gastrojejunostomy, and he himself has had a case in which one attack of hæmatemesis followed this operation. That some- 
times hæmorrhage does recur after gastro-jejunostomy, is not open to question, but such cases are rare, and I believe that the majority of them come under one of four categories. First, those in which malignant disease exists ; secondly, those in which a mechanical appliance has been used to effect the anastomosis ; thirdly, those in which the anastomotic opening has become, or originally was, inefficient; fourthly, those in which hæmorrhage occurs from the mucous membrane in the line of suture.

In the museum of St. Bartholomew's Hospital is a specimen (No. 1951C), which appears to show that a fatal hæmorrhage may ensue from the granulating area caused by the pressure of the button on the tissue surrounding the opening.

Some years ago I was present at the autopsy on a man who had died from hæmatemesis fourteen days after gastrojejunostomy. I was able to demonstrate that water injected into the stomach did not escape through the efferent opening, and I have seen two other cases in which a similar condition obtained. In some of the recorded cases it is stated that regurgitant vomiting accompanied the hæmorrhage, a fact suggesting obstruction of the anastomotic opening. That with a distended stomach hæmorrhage should recur, is not surprising.

I believe that in the majority of cases in which hæmorrhage occurs after gastro-jejunostomy, the bleeding is not from the ulcer but from the cut mucous membrane round the anastomosis. There is a specimen in the St. Bartholomew's Hospital Museum (No. 1951a ${ }_{3}$ ) from a patient who died from hæmorrhage after gastro-jejunostomy, in which it is clear that the bleeding was from the anastomosis.

Such cases do not prove that the principle of treatment by gastro-jejunostomy is wrong, but that the method of carrying it out is faulty. Probably the cases are very few 
in which hæmorrhage from the ulcer has recurred after gastro-jejunostomy. Excision is a more serious operation, especially in a patient suffering from the effects of loss of blood, and even after this method of treatment, recurrence of the hæmorrhage is not unknown (see Plate XXII, Fig. 52).

The old rule of surgery, to cut down upon and to tie the bleeding vessel, requires modification in the light of modern experience. That we must look our enemy in the face, is an aphorism which sounds well, but one which may lead to injudicious surgery, if applied in every case. The indirect method of treatment by gastro-jejunostomy is simpler, and the proportion of failures is exceedingly small. There is no doubt, that in many instances, excision of the ulcer does effect a cure, but it is not readily applicable when the ulcer is on the posterior wall of the stomach, the situation of most ulcers which bleed. Further, it may prove ineffectual when a second bleeding, but undiscovered, ulcer exists. Ligature of the bleeding-points en masse, while satisfactory in many instances, appears to be less dependable than gastrojejunostomy.

Supposing, that after opening the stomach, the bleedingpoint be not discovered, what is to be done? Clearly, if the hæmorrhage has been repeated in such quantity, or so frequently, that an operation is justifiable, gastrotomy alone is not likely to prevent its return. If the hæmorrhage be such as to necessitate operation, the probability is that it will recur if nothing more than an exploratory operation be performed. Under such circumstances it appears to me to be better practice to perform gastro-jejunostomy at once, without opening the stomach to search for the bleedingpoint.

Gastro-jejunostomy possesses these advantages: that it is applicable equally to cases of acute, or chronic ulcer, it renders exploration of the stomach unnecessary, and it can be 
performed in very short time. Further, having decided that operation is necessary, we start with a definite procedure in view, whereas an attempt to discover the exact source of bleeding may lead to a prolonged operation on a patient little able to bear it. Human nature being what it is, it is difficult to stay our hand, if we do not discover at once the source of the bleeding which we seek. The rules which I have formulated for my own guidance in these difficult cases are as follows :

In hæmorrhage from an acute ulcer, erosion, or exulceratio simplex - that is, when the onset of bleeding is sudden and history of previous gastric ulcer is absent-first give a thorough trial to absolute rest in bed, ice applied to the abdomen, Trippier's hot-water injections by the rectum, and avoidance of food by the mouth for at least four or five days. If a second profuse hæmorrhage occur, perform gastro-jejunostomy, provided it be quite clear that the second hæmorrhage has not been brought on by want of absolute rest, by too early stimulation, or by too early administration of food. It is advisable to delay operation until the patient has recovered from the collapse induced by the hæmorrhage. In hæmorrhage from a chronic ulcer-that is, in cases with a definite history of pain after food, vomiting, and possibly previous slighter attacks, of hæmatemesis-perform gastrojejunostomy after one severe attack, or after several slighter attacks of hæmatemesis, if the loss of blood be causing serious anæmia.

As I have indicated previously (p. 181), my belief is that in duodenal ulcer, most commonly the hæmorrhage comes, not from the ulcer, but from the mucosa of the stomach. The principles of treatment are the same as in hæmorrhage from a gastric ulcer, and the method of applying them identical. There is, therefore no necessity to add anything to what is contained in the preceding paragraphs. 


\section{REFERENCES}

Andrews and Eisendrath, Annals of Surgery, Oct. 1899.

Chiari, Prager Med. Woch., 1882, vii. p. 489.

Dieulafoy, Clinique Méd. de l'Hôtel Dieu, 1899, pp. 1-62.

Fenwick, Soltau, Trans. Path. Soc., 1892, and Ulcer of Stomach, 1900, pp. 59 and 182.

Korte, Centralblatt f. Chir., 1897, p. 69.

Mikulicz, Centralblatt f. Chir., 1897, p. 69.

Moullin, Mansell, Brit. Med. Jour., 1903, ii. p. 985.

Murchison, Trans. Path. Soc., 1870, xxi. p. 162.

Paterson, H. J., Gastric Surgery, 1906, p. 53.

Roux, Congress of French Surgeons, 1893.

Savariaud, De l'Ulcère Hémorrhagique, Paris, 1898. 


\section{CHAPTER XIII}

\section{THE COMPLICATIONS OF GASTRIC AND DUODENAL ULCERS-continued}

\section{CHRONIC DILATATION OF THE STOMACH}

Chronic dilatation of the stomach may arise from gastric or duodenal ulcer in two ways: (i) cicatrisation of an ulcer situated at, or near, the pylorus may cause definite mechanical obstruction to the exit of the gastric contents, or (ii) the presence of an ulcer near the pylorus may cause spasm of the pylorus, which may persist even after the ulcer has healed.

During the process of healing, a gastric ulcer, like any other ulcer, contracts. This contraction causes certain changes in the stomach which lead to well-marked symptoms, depending on the situation of the cicatrix. If the ulcer be at, or near the pylorus, cicatrisation may produce pyloric stenosis, while if the ulcer be in the body of the stomach, the constriction produced by the cicatrisation of the ulcer may divide the stomach into two portions, communicating by means of a narrow isthmus-a condition known as "hourglass stomach."

Pyloric stenosis may be caused also by pressure from without, as, for example, by the pressure of a tumour growing from the liver or pancreas, or by inflammation of a neighbouring organ, as when an inflamed gall-bladder becomes adherent to the duodenum, with the result that the duodenum also becomes infiltrated with inflammatory products.

The symptoms of pyloric obstruction, dilatation of the stomach, vomiting, and peristaltic movement of the stomach visible through the abdominal wall, form a clinical feature 
which is recognised readily. The nature of the vomiting is characteristic. It does not occur after meals, nor necessarily every day. After food there is a sense of discomfort which increases gradually with each succeeding meal, until at last relief is obtained by vomiting. Sometimes the quantity vomited is enormous, and the vomit consists of fermented food mixed with much mucus. Often articles of food taken some days previously are found in the vomit.

The extent of the vomiting does not depend entirely upon the degree of narrowing of the lumen of the pylorus. I have seen profuse vomiting with a pylorus having quite a fair lumen. I think the important factor is the extent of the interference with the contraction of the circular fibres, by the formation of fibrous tissue. When the musculature of the pylorus is replaced by fibrous tissue, the wave of peristaltic contraction, originating in the stomach, is arrested at the pylorus, and so the emptying of the stomach is imperfect.

The outline of the dilated stomach usually is plainly visible through the abdominal walls, and waves of peristalsis may be seen and felt passing across the upper half of the abdomen from left to right. The extent of the dilatation can be ascertained by palpation and percussion, after distending the stomach with air.

The most important diagnostic sign of pyloric stenosis is the presence of food in the stomach in the early morning. If 340 c.c. (12 oz.) of milk be given overnight, on passing the stomach tube ten hours later, 200 c.c. or more of fluid containing food may be recovered. If some raisins or other easily recognisable food be given with the milk, some of them will be found in the stomach next day.

If the stomach be empty in the morning we can be certain that pyloric stenosis is not present.

The treatment of organic pyloric stenosis is gastrojejunostomy. 


\section{CHAPTER XIV}

\section{THE COMPLICATIONS OF GASTRIC AND DUODENAL ULCERS-continued}

\section{HOUR-GLASS STOMACH}

By the term "hour-glass" stomach is meant a stomach which is divided into two cavities by a constriction. It is a serious complication of gastric ulcer. Not infrequently the patients suffering from it are so emaciated, that they are supposed to have advanced malignant disease, a diagnosis supported by the gastric findings, which resemble closely those of gastric carcinoma. The patient, therefore, may be considered beyond the reach of surgery, and so is allowed to die unrelieved. Such an error is one to be guarded against, as there are few conditions in which relief is so immediate, and so complete, as it is by the surgical treatment of hourglass stomach.

The first accurate description of the condition was given by Morgagni. Usually the constriction is in the middle third of the stomach, sometimes near the pylorus, or more rarely, near the cardiac end. In most of the specimens which I have examined, the communication between the two pouches has been smaller than the external appearance of the stomach would suggest. Indeed, it is of practical importance to recognise, that on opening the abdomen, an examination of the external surface of the stomach may give little indication of the serious constriction within. In one patient upon whom I operated, there was only a slight puckering on the anterior wall of the stomach, but internally, 
an annular stricture so tight, that it admitted only the tip of my little finger. (See Plate XXIV, Figs. 55 and 56.)

In the majority of the specimens and recorded cases, the greater curvature is drawn up towards the lesser, but in one case figured by Schwarz, the lesser curvature is drawn down towards the greater.

The Pathology of Hour-glass Stomach.-Two varieties of hour-glass stomach have been described-the congenital and the acquired; but Mayo Robson, and Moynihan have expressed a doubt as to the existence of hour-glass stomach as a congenital deformity. A perusal of the recorded cases, and an examination of the specimens in the London museums, have led me to a similar conclusion. The cases brought forward by Carrington have been accepted generally as undoubted examples of congenital deformity, but in all of them there were either ulcers, or scars of previous ulcers, or adhesions. The presence of ulcers or scars was noted also in the cases recorded by Roger Williams, Hirsch, and Hudson. Sievers records a case of " congenital" hour-glass stomach in a woman, aged thirty-eight years, who died from suppurative peritonitis due to perforation of a gastric ulcer in the pyloric pouch. No macroscopic or microscopic evidence of pathological change could be discovered in the constricted area. A very small ulcer, however, may lead to very extensive cicatrisation, and the scar of an ulcer is so difficult to detect, hidden as it may be in folds of the mucous membrane, that unless sections of every portion of the constriction be examined, the scar of a healed ulcer may be overlooked easily. (See Plate XXIII, Fig. 53, p. 154.)

As regards Hudson's hypothesis, that the ulceration in these cases is secondary, it is at least suggestive, that in Sievers' case the ulcer which perforated was on the distal side of the constriction and not in the cardiac pouch, where the stagnation and irritation of food must have been greater. 


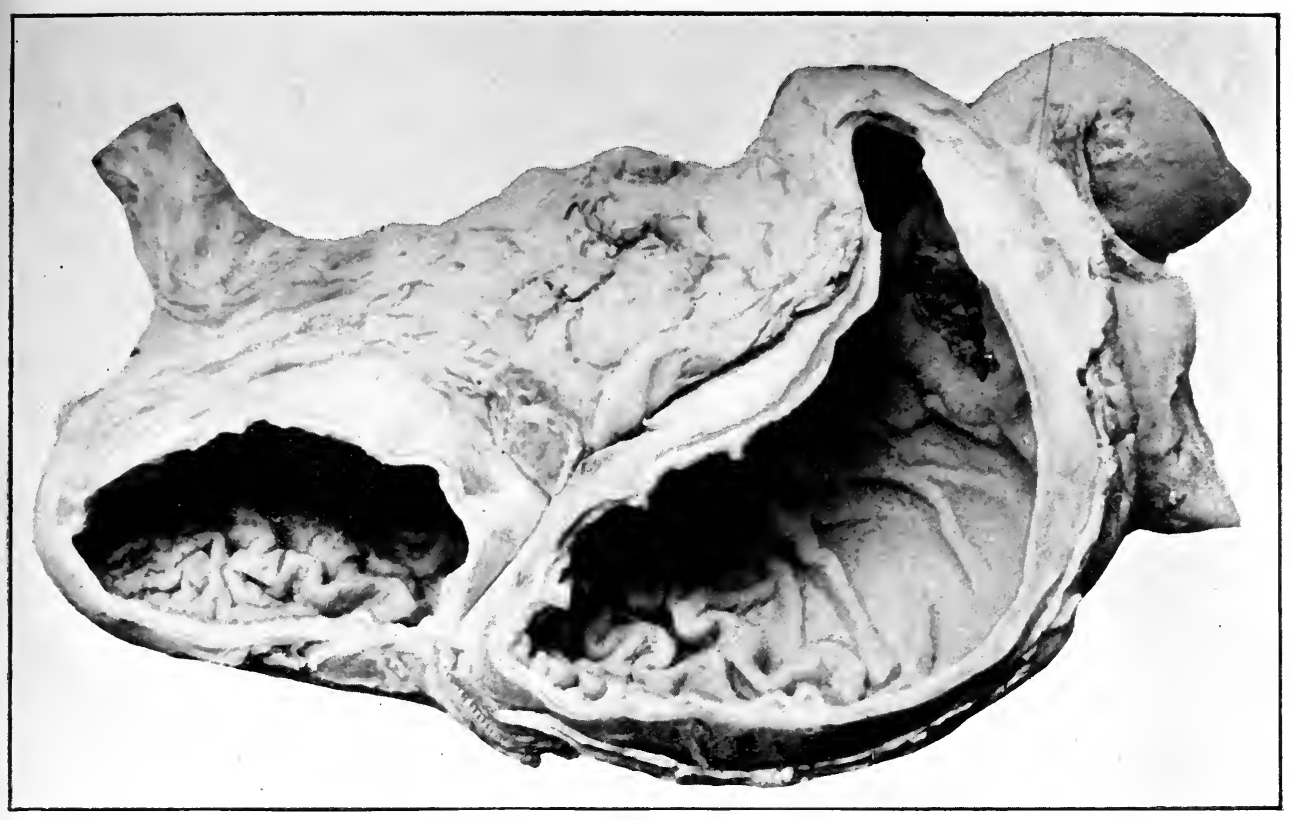

FIG. 55.-The interior of an hour-glass stomach viewed from behind.

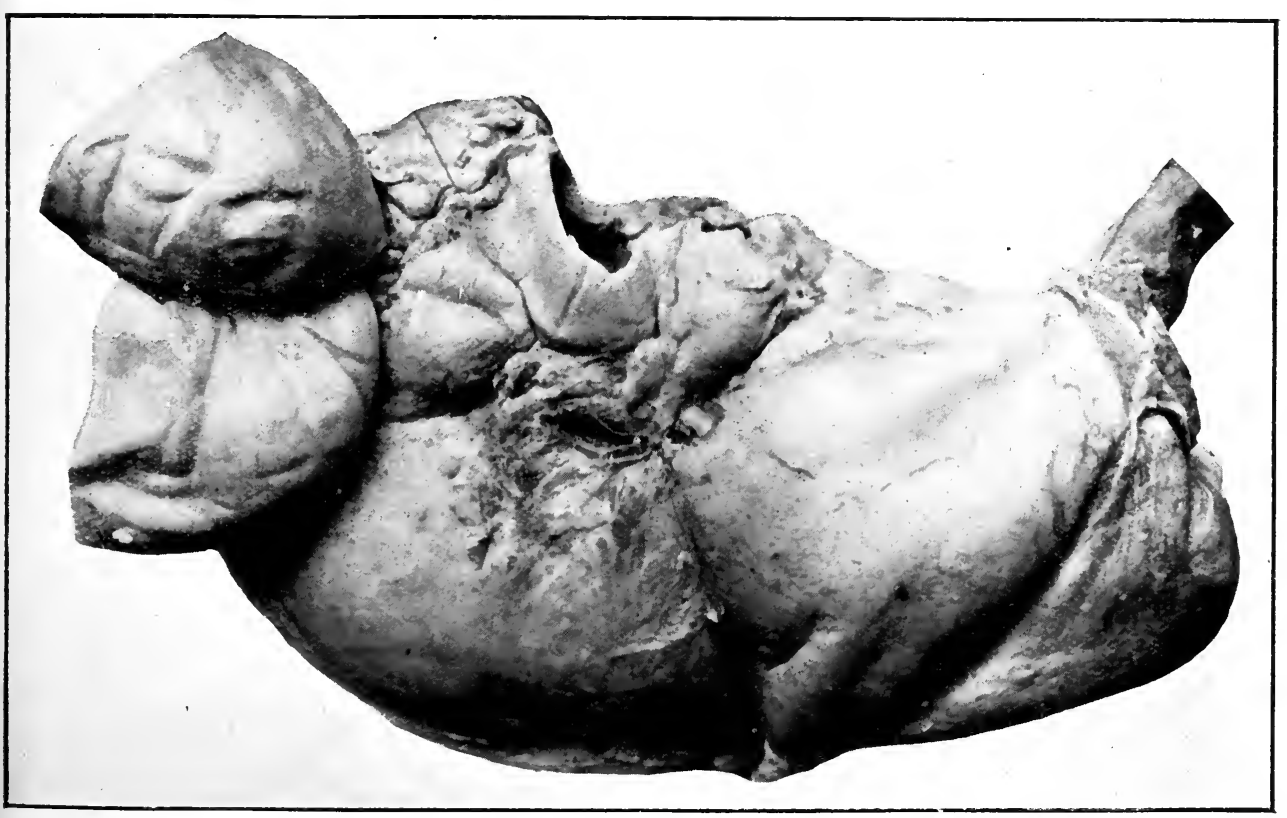

FIG. 56.-The front view of the exterior surface of the specimen shown in preceding figure. Note how little evidence there is of the marked constriction which exists within. 
It is a striking fact, that in all the cases which $I$ have been able to discover, symptoms were absent during early life. Thus, in Sievers' case the patient was quite well until the age of twenty-six years, and in Hirsch's case gastric symptoms began at the age of sixteen years, and increased. gradually after the age of forty years, so that during the last ten years of her life the patient was unable to take solid food. The absence of symptoms in early life is difficult to reconcile with the hypothesis of a congenital origin, while the appearance of symptoms between the ages of fifteen and thirty years is strongly suggestive that gastric ulcer is the causal factor. It is, too, most significant, that up to the present time, so far as I can discover, no instance of an hour-glass stomach in a fotus has been recorded. ${ }^{1}$ Possibly the following observation may explain some of the specimens of so-called congenital cases. I was present at a post-mortem examination when an apparently typical specimen of an hour-glass stomach was discovered. Through an oversight the specimen was not put into formalin at once. On examination of the specimen next day, I found that all trace of the constriction had vanished, and, presumably, what had appeared to be constriction was really only a muscular contraction which had persisted for some time after death. Had the specimen been placed in some hardening reagent immediately, doubtless it would have been preserved as a typical example of congenital hourglass stomach.

The cases of Hochenegg, Doyen, and Cumston are described as congenital, but in all of them there was evidence of ulceration. In Doyen's case the stomach had become

1 Sandifort (Observationes anatomico-pathologae, published 1779 , liber iii., p. 11) figures a stomach with a slight narrowing midway between the cardiac and pyloric ends, which was obtained from a fœtus. The narrowing is very slight, and in the absence of any examination of the interior of the organ, it can hardly be recognised as an hour-glass stomach. 
adherent to the anterior abdominal wall, and on separation of the adhesions a perforation of the stomach was discovered.

In some instances the stomach is divided by two constrictions into three pouches, forming the so-called "trifid stomach." Of this variety I have had two examples.

Hour-glass stomach may be the result of :

(i) Cicatrisation of a gastric ulcer.

(ii) Puckering due to perigastric adhesions.

(iii) Adhesions of the stomach to the anterior abdominal wall following perforation of a gastric ulcer.

(iv) Contraction of a gastric carcinoma.

The Symptoms and Signs of Hour-glass Stomach.-The most constant symptom of hour-glass stomach is pain. In all of my cases pain has been marked, and its presence is noted in 93 per cent. of the recorded cases. Vomiting is present in 85 per cent. of the cases, while gastrorrhagia as evidenced by hæmatemesis or melæna, or by both, occurred in 31 per cent. of the recorded cases. In one of my cases the hæmorrhage was so severe as to prove almost fatal.

Usually, the onset of pain does not follow the ingestion of food immediately, but after an interval varying from half an hour to two hours. In the later stages of the disease the pain may be constant. It is " boring" in character, and is referred to the epigastrium, occasionally with radiation to the back. In the early stages of the disease heartburn (acid eructations) often is one of the symptoms.

The signs are those of dilated stomach which, in many of the cases, has been attributed to pyloric stenosis. If, however, the condition be suspected, there are certain signs which usually will enable the observer to make a correct diagnosis. The signs are :

I. If a definite quantity of water be introduced into the stomach by a tube, a portion of the fluid escapes into the 
pyloric pouch, so that only the fluid remaining in the cardiac pouch can be recovered. Wölfler, who called attention to this sign, said that "Some of the fluid introduced seemed to disappear as though it had flowed through a large hole."

2. If the stomach be washed out until the returned fluid is clear, after waiting a few minutes there may be a gush of dirty, offensive fluid, which has regurgitated from the pyloric into the cardiac pouch (Wölfler's second sign).

3. The stomach resonance is percussed carefully, and then a seidlitz powder is given in two separate halves. After an interval of thirty seconds, there is considerable increase in the resonance of the upper part of the stomach, while the lower part remains unaltered. If the abdomen be watched for a few seconds, the pyloric pouch sometimes may be observed to fill gradually and become prominent (Moynihan's sign).

Personally, I distend the stomach with air by means of an inflater, in preference to the use of the seidlitz powder.

4. Sometimes after apparent emptying of the stomach by means of a tube, there is still a splashing sound on palpating the upper part of the abdomen. This sign is known as "paradoxical contraction." The splashing sound is produced in the pyloric pouch, which, of course, is undrained by the passage of the stomach tube into the cardiac pouch.

5. Usually examination of the stomach by the X-rays, after administration of a bismuth meal, will show clearly the presence of an hour-glass stomach. This is a valuable positive sign ; too much reliance must not be placed upon it if it be negative.

Of all these signs the most valuable are, the washing out test and the distension test (Nos. 1 and 3).

Gastric Analysis.-The gastric findings are those of chronic gastritis. I have never found any free hydrochloric acid in a case of hour-glass stomach. I have not seen this 
mentioned by previous writers. It is an important truth, as the absence of free hydrochloric acid leads frequently to a diagnosis of malignant disease.

At first sight the absence of free hydrochloric acid is difficult to explain. The oxyntic cells are in the cardiac end of the viscus, and therefore we should expect that free hydrochloric acid would be secreted as usual. Possibly the explanation is that the gastric hormone, which is secreted in the pyloric pouch, does not reach the cardiac end of the stomach, and so the oxyntic cells are not stimulated into activity.

The Diagnosis of Hour-glass Stomach.-When the constriction is in the pyloric half of the stomach, the dilated cardiac pouch is often mistaken for a dilatation of the whole stomach due to pyloric stenosis.

Usually Wölfler's two signs, already mentioned, supplemented by distension with air, will enable a correct diagnosis to be made. When the constriction is near the cardiac end of the stomach a correct diagnosis is less easy. The cardiac pouch may be so small that there is difficulty in swallowing, and also regurgitation of food, very soon after it is taken. Such symptoms suggest œesophageal obstruction. The passage of an œsophageal bougie will exclude this condition, if the bougie can be passed more than sixteen inches from the teeth.

Many of the patients are so emaciated and cachectic as to suggest gastric carcinoma. A long history and severe pain suggest inflammatory rather than malignant disease, and in my experience the total acidity in hour-glass stomach is not so low as it is in a case of carcinoma of such long standing.

In hour-glass stomach of inflammatory origin usually the total acidity is about forty or fifty, while if the disease be malignant we may expect a total acidity of twenty to thirty, or even less. 
The Treatment of Hour-glass Stomach.-The following procedures may be adopted in the surgical treatment of hour-glass stomach :

r. Gastro-jejunostomy.

2. Gastroplasty.

3. Gastro-gastrostomy.

4. Stretching the constriction.

5. Partial gastrectomy.

In addition to one or more of these operations, it may be necessary also to divide gastric adhesions (gastrolysis).

In operating for hour-glass stomach it is most important to examine carefully the whole stomach before deciding what is to be done, otherwise the presence of a third pouch, or of pyloric stenosis, may be overlooked.

Gastroplasty is the simplest method of dealing with hour-glass contraction, but it is open to the same objection as pyloroplasty for pyloric stenosis-namely, the frequency with which relapse ensues. In at least 25 per cent. of the patients who have recovered, either no relief has followed, or relapse has occurred subsequently. This happens especially when there is any active ulceration at the time of operation, while, even if there be no active ulceration, recurrence is not improbable for two reasons. First, gastroplasty is not a preventive of gastric ulcer, and does not modify the conditions predisposing to gastric ulceration, which presumably are present in these cases. Secondly, adhesions frequently are present which impair the motility of the stomach and keep up a certain degree of gastric stasis. This leads to hyperchlorhydria, which in its turn causes, or at least predisposes to, and keeps up, ulceration.

The same criticism applies to the operation of gastrogastrostomy. At least 30 per cent. of the patients on whom this operation has been performed, either have obtained no relief, or have relapsed. It appears, therefore advisable, 
that if gastroplasty or gastro-gastrostomy be the procedure adopted, gastro-jejunostomy should be performed in addition.

In my earlier cases I performed the double operation of gastroplasty and gastro-jejunostomy, but I think that in most cases this is unnecessary, and I now perform a gastrojejunostomy in the proximal pouch. If pyloric stenosis exist as well as hour-glass stomach, my practice is to perform a double anastomosis by making a connection between the jejunum and both the cardiac and the pyloric pouches, as was suggested by Weir and Foote.

A simple gastro-jejunostomy, or in cases associated with pyloric stenosis, a double gastro-jejunostomy, has the merit that it does not necessitate cutting into, or operating on, the diseased area. In principle it seems the best method, and I consider it to be the method of choice. In cases of malignant hour-glass stomach, if practicable, partial gastrectomy should be performed.

Prognosis. - The prognosis after operation for hour-glass stomach is good, provided a gastro-jejunostomy be performed.

Owing to the extreme emaciation of many of those who are suffering from hour-glass stomach, the mortality rate is perhaps slightly greater than in gastro-jejunostomy for simple ulcer or for pyloric stenosis. Operation for hour-glass stomach should be a rare operation, as the patients should be treated surgically long before the ulcer has progressed to the extent of producing marked constriction of the stomach.

I have operated on fourteen patients suffering from hourglass stomach, and so far have been fortunate in that all of them have recovered.

My first operation was performed in 1902, and all the patients except one have remained quite well ever since. The one exception remained quite well for five years, and then had symptoms of intestinal obstruction. I reopened 
the abdomen and found that both the jejunum and transverse colon had been obstructed partially, at the site where they crossed one another. I performed an entero-anastomosis and an ileo-sigmoidostomy, and since then (nearly three years ago) the patient has remained in perfect health. In all my cases a gastro-jejunostomy was performed, in some of them combined with gastroplasty.

\section{REFERENCES}

Blake, Annals of Surgery, Feb. 1903, p. 280.

Carrington, Trans. Path. Soc., 1882, xxxiii. p. 130.

Childe, Brit. Med. Jour., 1901, i. p. 695.

Doyen, Traitement chir. des affections de l'estomac, 1895, p. 308.

Gilford, H., Brit. Med. Jour., 1902, ii. p. 1527.

Hirsch, Virchow's Archives, 1895, cxl., No. 3.

Hudson, Brit. Med. Jour., 1886, ii. p. 973.

Jaworski, Wiener Klin. Woch., April 10, 1899.

Morgagni, Opera omnia, 1645.

Moynihan, B. G. A., Brit. Med. Jour., 1904, i. p. 413.

Paterson, H. J., The Practitioner, Nov. 1911, p. 642.

Robson and Moynihan, Diseases of the Stomach, 1904, p. 173.

Rowlands, R. P., Brit. Med. Jour., 1911, i. p. 669.

Schwarz, Wiener Klin. Woch., June 1896, p. 48.

Sievers, Berliner Klin. Woch., April 10, 1899, p. 15.

Watson, S., Annals of Surgery, July 1900, p. 85.

Williams, R., Jour. Anat. and Physiol., 1883, xvii. p. 460.

Wölfler, Beit. f. Klin. Chir., 1895, No. 13, p. 221. 


\section{CHAPTER XV}

\section{THE COMPLICATIONS OF GASTRIC AND DUODENAL ULCERS-continued}

\section{GASTRIC TETANY}

Gastric tetany due to dilatation of the stomach was first recorded by Kussmaul, so recently as the year 1869 .

In the majority of cases the dilatation has been due to cicatricial pyloric stenosis. In a few instances the disease was in the first part of the duodenum (Reuvers, Baumberger, Dujardin-Beaumetz), in others, both the pylorus and the duodenum have been affected (Loeb, Müller).

In rare instances the obstruction of the pylorus has been due to malignant disease.

Often the early symptoms, pricking and numbness of the hands, pass almost unnoticed by the patient. Generally the first noticeable symptom is a tonic spasm of the muscles of the extremities. As a rule its onset is quite sudden, and is preceded by an attack of severe vomiting.

In 1902 I saw a typical case under the care of Dr. Soltau Fenwick. I will quote from my notes the description I wrote of the patient's condition during an attack: "Arms abducted, forearms flexed and strongly pronated. The wrists strongly flexed, the fingers drawn together, flexed at the metacarpo-phalangeal joint, the phalangeal joints extended. Thumb strongly adducted into the palm of the hand. Lower limbs fully extended, the ankles extended, and the soles of the feet adducted and rotated inwards. Some twitching about the mouth. Patient absolutely rigid all over, and unconscious." 
The attack lasted six hours, during which time the patient remained unconscious, while the temperature gradually rose to $102 \cdot 8^{\circ} \mathrm{F}$., the highest point being reached just as the attack was passing off.

A few days after the patient had recovered from the attack I performed an anterior gastro-jejunostomy. The patient has never had a day's illness since the operation ten years ago, and he has put on three stone in weight. The case is of interest as it is, I believe, the first in England in which gastro-jejunostomy was suggested and performed for gastric tetany. In Scotland, a few months earlier, Mr. Caird had performed gastro-jejunostomy in a similar case, but it was not reported until a later date, so that when Dr. Fenwick suggested gastro-jejunostomy in this case, he was unaware that this operation had been performed previously for gastric tetany. Before this, several cases had been treated surgically, but in all of them pyloroplasty had been performed.

Usually certain symptoms are present. These are :

(i) Trousseau's Symptom.-As the attack is passing off, pressure over the principal nerve-trunk or blood-vessels, sufficient to impede the venous or arterial circulation, will cause a recrudescence of the paroxysm.

(ii) Chvostek's Symptom.-A slight tap over the principal nerve of the part affected will cause spastic contraction of the muscles supplied by the nerve.

(iii) Erb's Symptom.-Great increase of electrical irritability to a faradic current.

(iv) Hoffman's Symptom.-Slight pressure over a sensory nerve may cause paræsthesia in the area supplied by the nerve.

As the attack passes off there is considerable pain and tingling in the limbs. Usually the first attack is followed within a short time by others. Occasionally an interval of several days, or even months, separates the first and second attacks. 
Slight albuminuria is an almost constant symptom, although no evidence of renal mischief has been discovered. The albuminuria is due, probably, to the excretion of some toxin, and is in favour of the view that the spasms are due to auto-intoxication, the result of abnormal chemical processes in the stomach (Bouveret and Devic). Marked tetany is a very serious complication of gastric ulcer, for about 70 per cent. of the cases recorded have ended fatally. At the same time it is a complication which ought not to occur. Prevention is better than cure, and the proper method of treating gastric tetany, is by dealing with all intractable gastric ulcers surgically. Fortunately, gastric tetany rarely is fatal during the first attack, but Marten has reported a case which proved fatal four hours after the first seizure, and Trevelyan has recorded a similar case. The dread of a second attack weighs on the patient's mind, and has a most depressing mental effect.

The treatment of gastric tetany, due to pyloric stenosis, is gastro-jejunostomy. The prognosis is good. Most of the patients have recovered, and have been relieved completely of their symptoms.

\section{REFERENCES}

Bamberger, Virchow-Hirsch Jahrsbericht, 1892, p. 174.

Berlizheimer, Berlin. Klin. Woch., 1897, p. 773.

Bouveret et Devic, Revue de Med., Feb. 1892, pp. 48, 97.

Caldwell, Brit. Med. Jour., 1902, i. p. 1603.

Chovstek, Zeit. f. Klin. Med., 1891, p. 489.

Collier, The Lancet, 1891, i. p. 1251.

Cunningham, J. H., Annals of Surgery, 1904.

Dickson, C., The Practitioner, 1903, p. 44.

Dujardin-Beaumetz, L'Union Médicale, xxxvii. p. 169.

Erb, Archiv f. Physiolog., iv. p. 271.

Fenwick, Soltau, Trans. Clin. Soc., xxviii. p. 13.

Gerhardt, Berlin. Klin. Woch., 1888, p. 74.

Hoffman, Deut. Archiv f. Klin. Chir., 1888, p. 603.

Jonesco and Grosman, Presse Médicale, July 1, 1905. 


\section{GASTRIC TETANY}

Kussmaul, Deut. Archiv f. Klin. Med., 1869, p. 455.

Loeb, Deut. Archiv f. Klin. Med., 1889, p. 95.

Marten, The Lancet, 1887, i. p. 74.

Muirhead, T. G., The Practitioner, Aug. 1904, p. 160.

Renvers, Berlin. Klin. Woch., 1884, p. 74.

Riegel, Diseases of the Stomach, p. 164.

Russell, Risien, Allbutt's System of Med., viii. p. 48.

Von Sievers, Berlin. Klin. Woch., 1898, p. 681.

Trousseau, New Sydenham Soc. Trans., 1868, i. p. 376. 


\section{CHAPTER XVI}

\section{THE PATHOLOGY, SYMPTOMS, AND DIAGNOSIS OF GASTRIC CARCINOMA AND SARCOMA}

\section{THE PATHOLOGY OF GASTRIC CARCINOMA}

Frequency.-The stomach enjoys the unenviable distinction of being affected more frequently with carcinoma than any other organ of the body. According to Häberlin, 41.5 per cent. of 27,511 cases of carcinoma that ended fatally were carcinoma of the stomach. D'Espere found that $44 \cdot 3$ per cent. of all cancers are situated in the stomach. According to the Report of the Registrar-General, during the years 1901 to 1907 there were 207,764 deaths from malignant disease in England and Wales, and 16.8 per cent. of these deaths were the result of cancer of the stomach. Probably a considerable number of the deaths from gastric carcinoma are not certified as such.

During fifteen years, out of 39,568 patients admitted into St. Bartholomew's Hospital, in 183 the diagnosis was cancer of the stomach. In other words, of every 216 patients admitted, one was suffering from gastric carcinoma, or just under 0.5 per cent., a proportion almost identical with that found by other observers. Of these patients 118 were men and 65 women. Dr. Soltau Fenwick gives the proportion as rather less than six men to four women.

Gastric carcinoma is a disease of late middle life. It is rare in young people. Of the 183 patients referred to above, 58.8 per cent. were between the ages of forty and sixty years. According to the Registrar-General's 

Plate XXV.

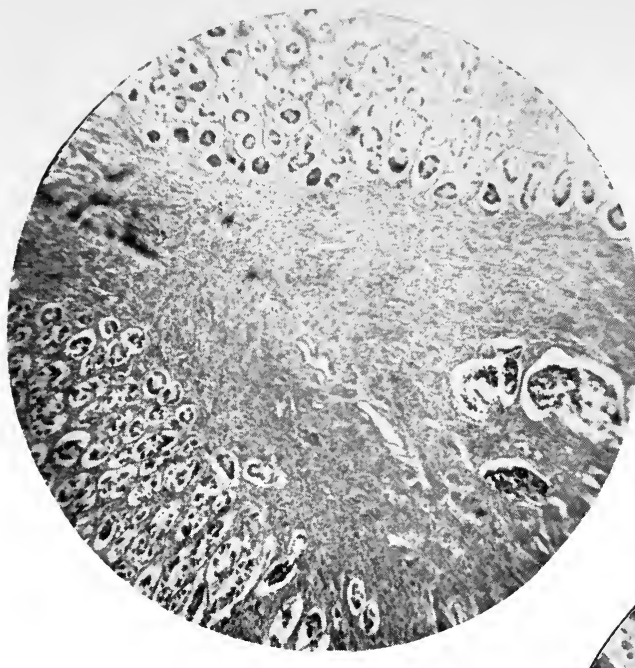

FIG. 57.-Microphotograph of spheroidal-celled carcinoma.
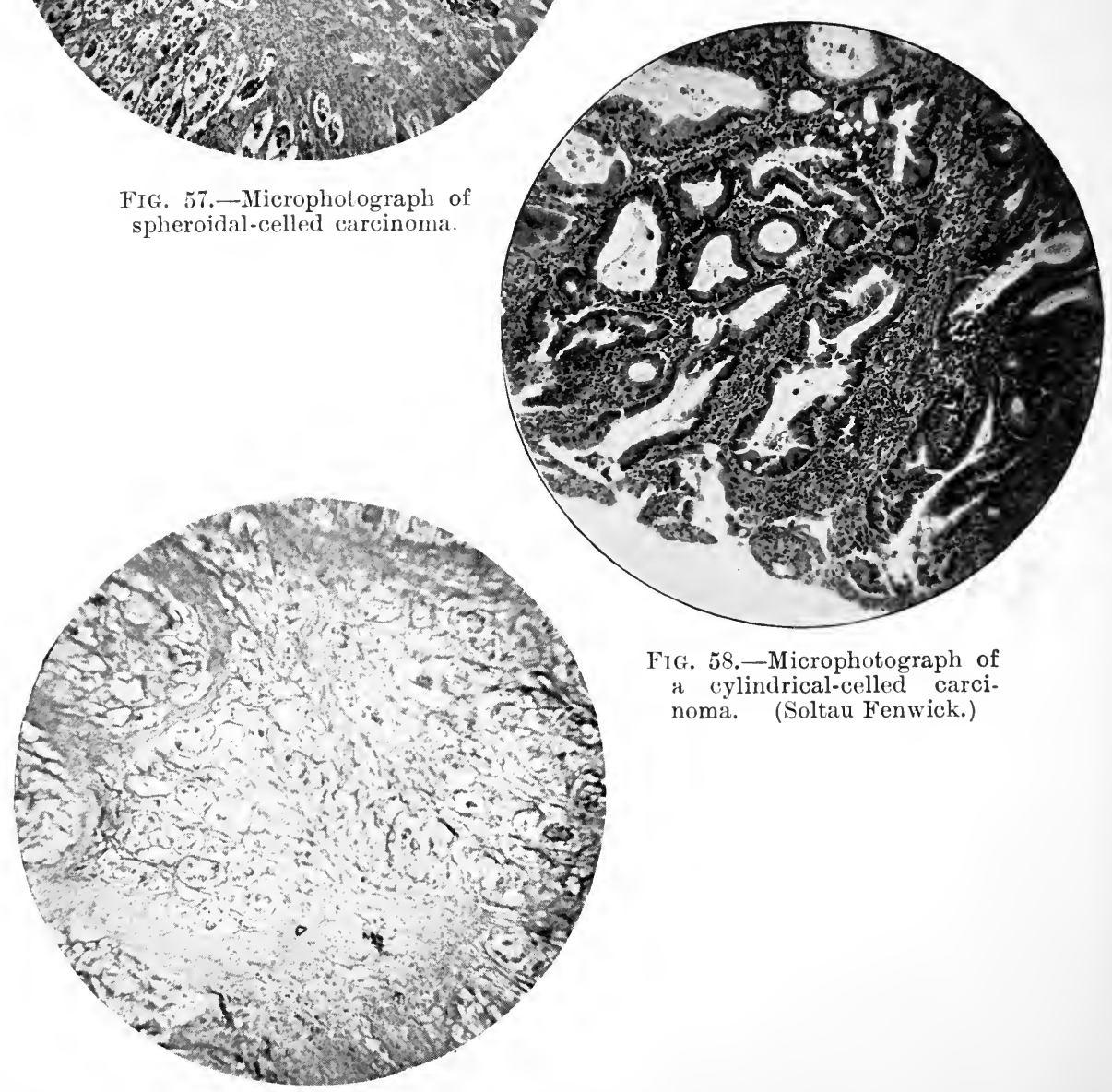

F1G. 58.-Microphotograph of i cylindrical-celled carcinoma. (Soltau Fenwick.)

FIG. 59.-Microphotograph of carcinoma, showing colloid degeneration. 


\section{GASTRIC CARCINOMA AND SARCOMA 207}

returns, 79 per cent. of the patients who died from malignant disease of the stomach during the years 1901-1907, were between the ages of forty-five and seventy-five years.

Pathological Anatomy.-Two types of carcinoma occur in the stomach, the spheroidal-celled, and the cylindrical-celled or adeno-carcimona. In both types colloid degeneration may occur (Plate XXV, Figs. 57, 58, 59).

Various estimates have been given as to the relative frequency of these two types. According to Cornil and Ranvier, cylindrical-celled is the more frequent. Dr. Fenwick concludes that the spheroidal-celled variety is more than twice as common as the cylindrical-celled. I have investigated the records of nearly two hundred cases in which an operation was performed, and an analysis of the records suggests that the cylindrical-celled variety is the more frequent. These cases, however, were not consecutive, and so cannot be accepted as conclusive evidence, but they do suggest that the cylindrical-celled type of carcinoma is more often amenable to radical treatment than is the spheroidalcelled.

At first the growth is limited to the gastric mucosa, but gradually it spreads to the submucous coat, and later extends to the peritoneum. The mucous surface of the growth ulcerates, and there is slight oozing of blood from the surface. In some cases the growth may destroy a large vessel, and so lead to alarming hæmorrhage.

The situation of the growth is important, not only from the point of view of surgical intervention, but because the symptoms depend, to some extent, on the part of the stomach affected.

The pylorus is more often implicated than any other part of the viscus. In the following table is shown the situation of the growth according to various authorities, together 
with the analysis I have made from 168 consecutive postmortem examinations at St. Bartholomew's Hospital.

Table showing the Position of Growth as calculated by Welch, Brinton, Habershon, Fenwick, and the AUTHOR.

In order to compare their statistics with mine I have expressed all the tables in percentages.

\begin{tabular}{|c|c|c|c|c|c|c|c|c|}
\hline & & & & Welch. & Brinton. & Habershon. & Fenwick. & Author. \\
\hline Pylorus & . & ${ }^{\circ}$ & & $60 \cdot 8$ & $60 \cdot 8$ & $51 \cdot 9$ & 58 & $61 \cdot 3$ \\
\hline Lesser curvature & . & . & . & $11 \cdot 4$ & $10 \cdot 5$ & $13 \cdot 9$ & $11 \cdot 5$ & $14 \cdot 2$ \\
\hline Cardiac orifice & . & . & & $8 \cdot 0$ & $10 \cdot 0$ & $12 \cdot 6$ & $9 \cdot 8$ & $5 \cdot 9$ \\
\hline Anterior wall & 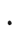 & . & & $2 \cdot 3$ & $3 \cdot 0$ & $6 \cdot 3$ & $2 \cdot 2$ & $5 \cdot 3$ \\
\hline Whole stomach & ${ }^{\circ}$ & . & • & $4 \cdot 7$ & $3 \cdot 6$ & $5 \cdot 0$ & $6 \cdot 0$ & $4 \cdot 7$ \\
\hline Central . & . & . & & $\ldots$ & $1 \cdot 1$ & $5 \cdot 0$ & $\ldots$ & $4 \cdot 1$ \\
\hline Posterior surface & . & . & & $5 \cdot 2$ & $3 \cdot 0$ & $\ldots$ & $5 \cdot 0$ & $4 \cdot 1$ \\
\hline Greater curvature & . & . & . & $2 \cdot 6$ & $3 \cdot 0$ & $1 \cdot 2$ & $2 \cdot 8$ & $\ldots$ \\
\hline Not stated & . & . & . & ... & $4 \cdot 7$ & $1 \cdot 2$ & ... & $\cdots$ \\
\hline
\end{tabular}

It is clear, then, that the growth far more frequently affects the pylorus and adjacent portion of the stomach than any other, the lesser curvature being the next most common seat of new growth.

When the growth is situated at the pylorus, it leads, sooner or later, to obstruction, which in some cases may be almost complete (Plate XXVI, Fig. 60).

In a few cases, the growth, instead of being limited to the pylorus and its immediate neighbourhood, infiltrates the wall of the stomach in its whole extent (Plate XXVI, Fig. 61). In these cases the stomach is sometimes small and densely hard, and from its shape is called "leather-bottle stomach."

When the peritoneum is invaded, the growth becomes adherent to neighbouring structures (liver, pancreas, colon, anterior abdominal wall), and when the whole thickness of the gastric wall is destroyed by ulceration, the base of the malignant ulcer is formed by the organ to which it has become adherent. When this is one of the hollow viscera, 


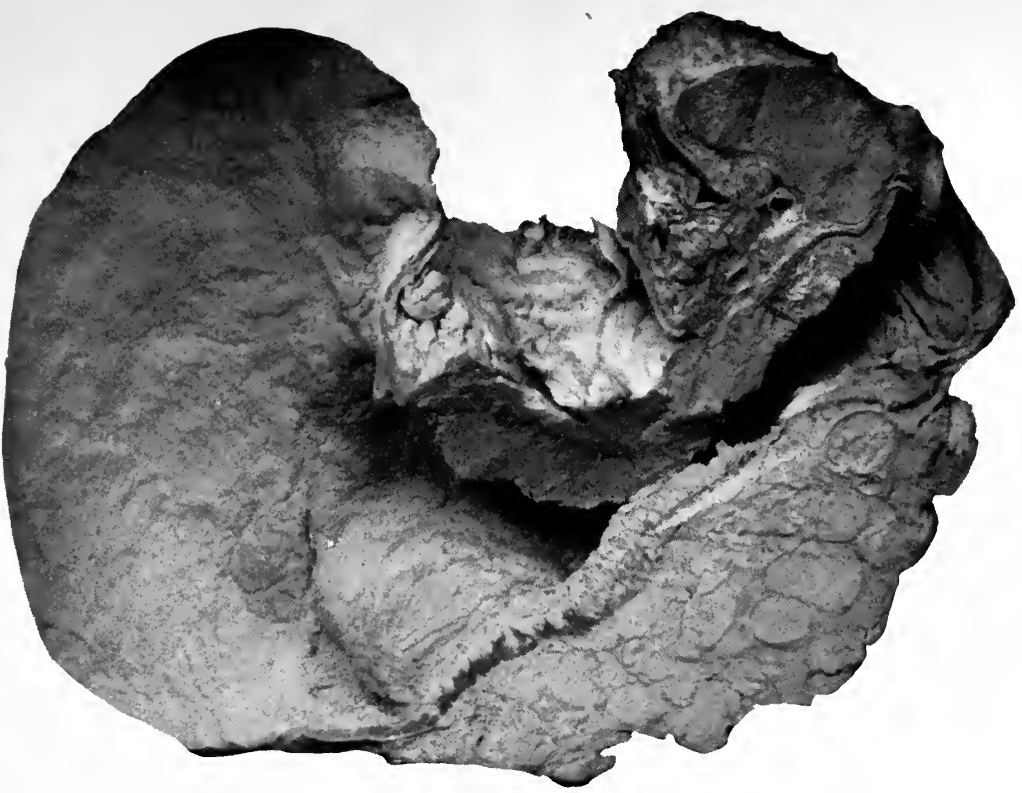

FIG. 60.-Cylindrical-celled carcinoma of the pylorus which has resulted in stenosis. Viewed from behind. (Soltau Fenwick.)

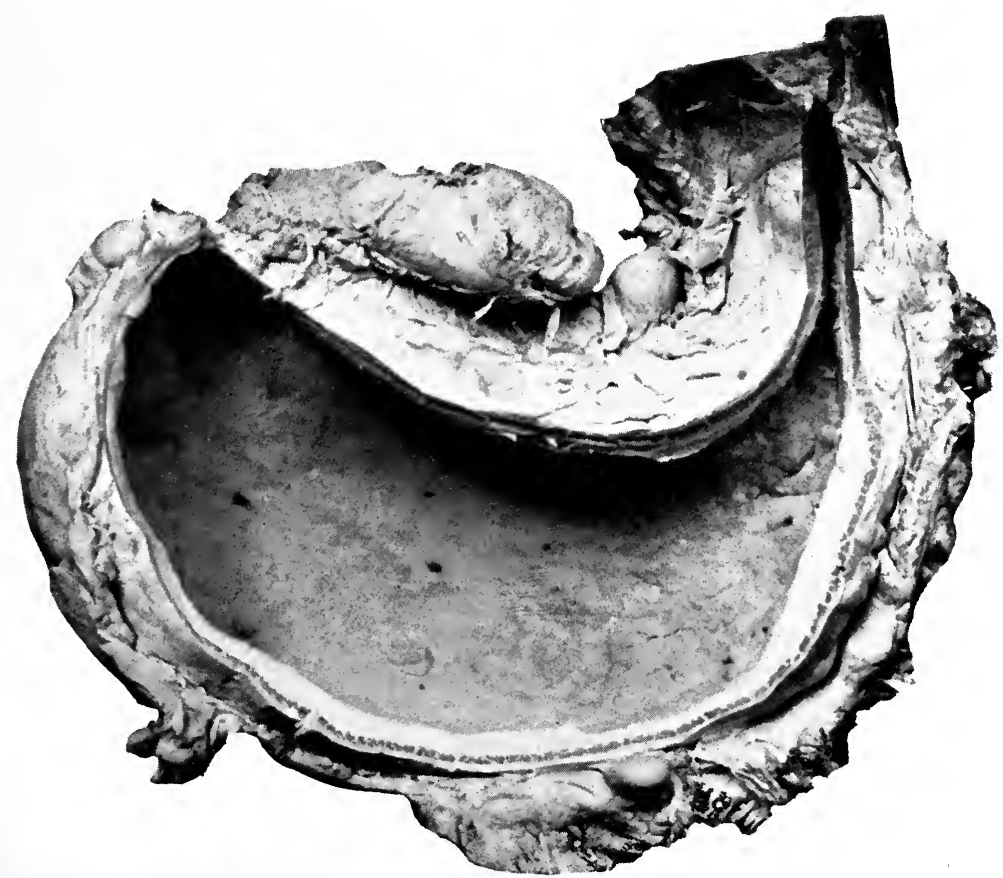

FIG. 61.-A stomach viewed from behind, showing diffuse infiltration by scirrhus carcinoma, with enlarged glands along the curvatures. (Soltau Fenwick.) 

a fistula may be formed between the stomach and the viscus to which it has become adherent (e.g. gastro-colic fistula).

The Lymphatic Glands in Gastric Cancer.-The stomach is supplied richly with lymphatic vessels. According to Cuneo the lymphatic spaces between the gastric glands are intercommunicating, form large spaces in the mucosa, pass

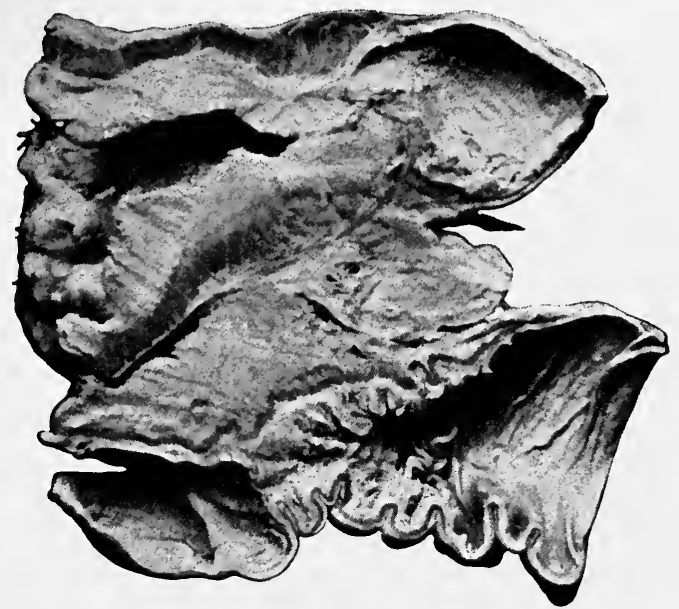

Fra. 62.-Colloid carcinoma of the pylorus invading the transverse colon. Specimen viewed from behind. (Soltau Fenwick.)

through the muscular coats, collect in the subserous layer, and enter lymphatic channels just under the peritoneum.

The lymphatic vessels of the stomach form three principal groups.

1. Those passing upwards towards the lesser curvature, which drain the upper half of the pyloric two-thirds of the stomach.

2. Those passing downwards towards the greater curvature, which drain the lower half of the pyloric two-thirds of the stomach.

3. Those passing across the fundus, which they drain, enter the gastro-splenic omentum and terminate in the glands of the hilum of the spleen. 
The glands into which the lymphatic vessels from the stomach drain, may be divided into four groups (see Fig. 63).

(a) The glands along the lesser curvature (gastro-hepatic group), situated in the gastro-hepatic omentum. Their efferent vessels pass backwards near the cardiac orifice and enter the glands around the coliac axis.

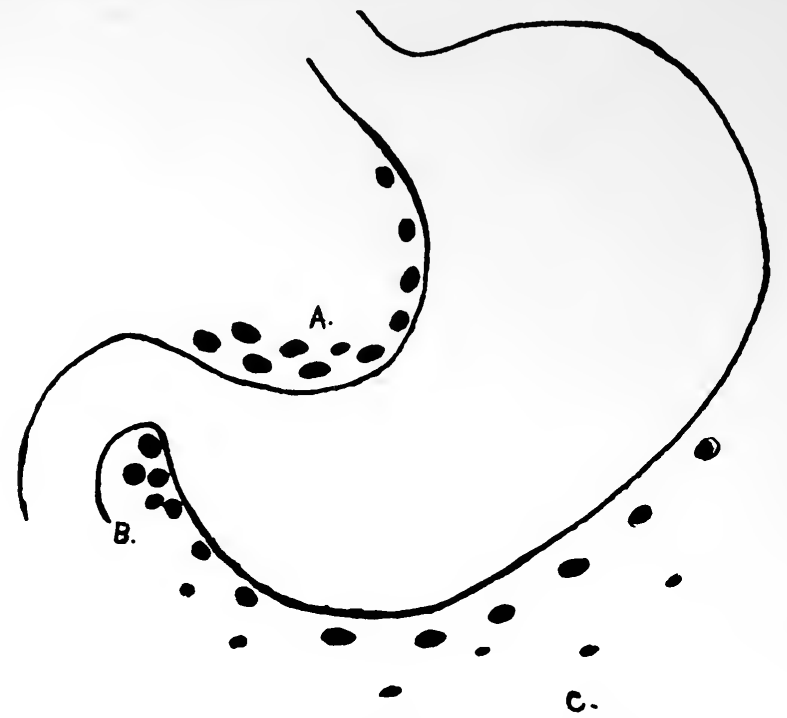

FIG. 63.-Diagram to show lymphatic glands of stomach.

$A$. Glands of lesser curvature (gastro-hepatic glands).

$B$. Sub-pyloric glands.

C. Glands of greater curvature (gastro-colic glands).

(b) The glands along the lower border of the pylorus (sub-pyloric group). Their efferent vessels pass into the cœliac glands.

(c) The glands behind the pylorus, around and over the head of the pancreas (retro-pyloric group). Their efferent vessels pass to the coliac glands.

(d) The glands along the greater curvature (gastro-colic group), situated in the gastro-colic omentum along the lower border of the stomach. Their efferent vessels pass into the glands around the cœliac axis. 


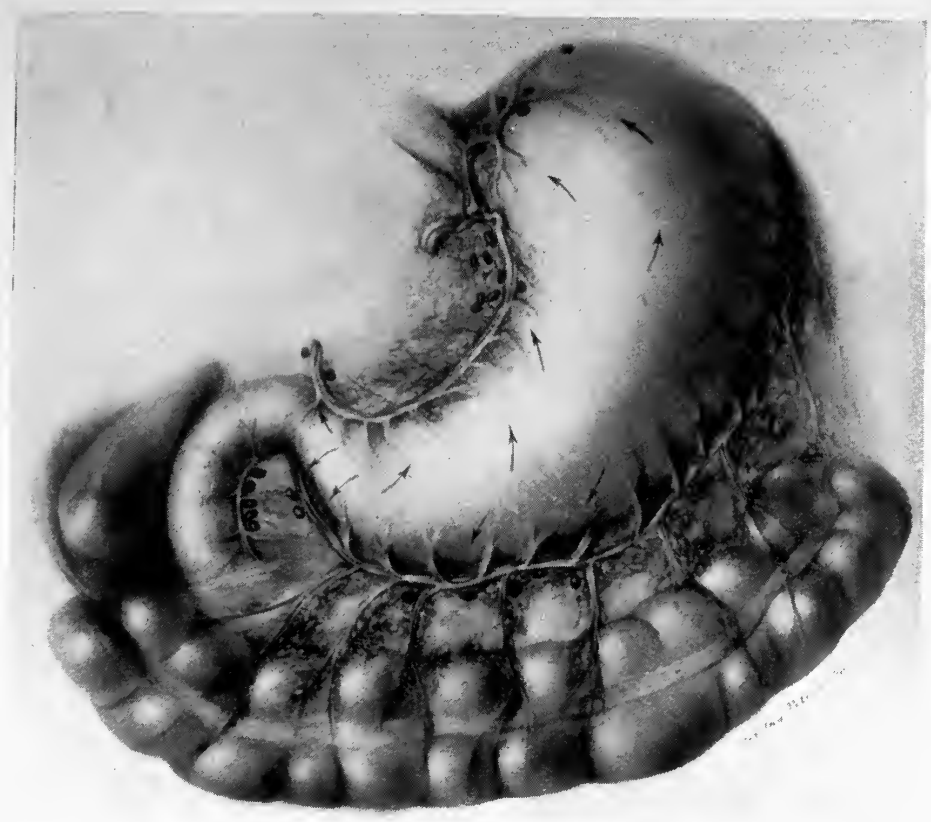

FIG. 64.- The stomach and transverse colon, showing the areas drained by the different groups of lymphatic glands. The arrows show the direction of the lymph current. (W. J. Mayo.)

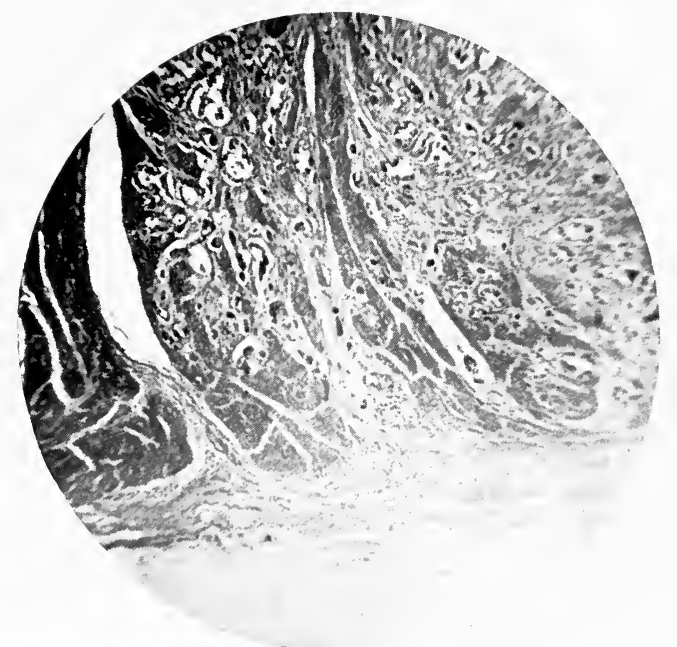

FIG. 65.-Microphotograph of a section cut beyond the area of induration in a case of gastric carcinoma, showing islets of growth infiltrating the muscular tissue. 

A practical point, the importance of which will be indicated later, is, that the glands along the greater curvature communicate with the glands lying along the upper border of the transverse colon.

As carcinoma usually is situated close to the pylorus at the lesser curvature, the gastro-hepatic glands are the first to become enlarged; sooner or later all the groups above-mentioned may become affected. The circumstance, that the glands are enlarged, does not necessarily mean that they are the seat of carcinomatous deposit; the enlargement may be due to inflammatory infiltration. Quite small glands may contain carcinomatous deposit, while glands of considerable size may be simply inflammatory, a very important point in considering the advisability of resection. In carcinoma of the cardiac orifice the infection of the lymphatic glands of the stomach is slight, but the deep cœliac glands may be implicated at an early stage of the disease. A very valuable paper has been published recently by Dr. MacCarty on the lymphatic glands in stomach cancer. His conclusions are as follows :

A. Negative Conclusions :

(i) The size of regional lymphatic glands bears no apparent relation to the size of the primary lesion in the stomach.

(ii) The size of a lymphatic gland is no criterion of the presence or absence of carcinoma.

(iii) Gross diagnoses of lymphatic glands are of no value, except in advanced carcinoma of the glands.

(iv) The duration of symptoms bears no apparent relation to the size and extent of involvement in the lymphatic glands.

(v) The average age at operation and sex bear no direct relation to the glandular involvement.

B. Positive Conclusions :

(i) The average loss of weight increases with the increase in extent of glandular involvement. 
(ii) The immediate hospital post-operative mortality is in direct proportion to the amount of glandular involvement.

(iii) The subsequent mortality is in direct proportion to the amount of glandular involvement.

(iv) Carcinomatous glandular involvement is very often microscopic.

(v) The surgeon who desires to treat early carcinoma must depend upon the microscope in the hands of an experienced pathologist for early carcinomatous lymphatic involvement.

(vi) The diagnosis of early carcinomatous involvement requires extensive experience in the study of so-called precarcinomatous reaction of lymphatic glands.

The Spread of Gastric Carcinoma.-From a surgical point of view, knowledge of the manner and direction in which the disease spreads, is of considerable importance.

Gastric carcinoma spreads in three ways :

(i) By Direct Continuity.

(ii) By Contiguity, or Invasion of Surrounding Structures.

(iii) By Dissemination through the Lymphatics.

(i) By Direct Continuity.-It is a curious fact, to which attention was directed first by Brinton and Rokitansky, that cancer of the stomach rarely spreads far into the duodenum. This is in striking contrast to the course of carcinoma of the cardiac orifice, as in this situation, the growth almost invariably spreads directly into the osophagus.

Another practical point in connection with extension by continuity is, as shown by Czerny and Cuneo, that islets of cancer-cells may be found in the submucous coat beyond the area of infiltration. This is seen well in the microphotograph (Plate XXVII, Fig. 65), which is taken from a section half an inch beyond the indurated area. A group of cancercells is seen infiltrating the muscle.

In order to be certain of removing the disease completely, 
the section should be made at least two inches beyond the indurated area.

The disease spreads along the lines of the lymph current, and as the greater number of lymphatic vessels run towards the lesser curvature, it follows that the growth extends in this direction. Thus the lymphatic glands along the lesser curvature may be affected by direct continuity. Consequently, it is of little use to dissect them out; they must be removed in one mass, together with the portion of stomach wall to which they are attached. If this be not done, the tributary vessels will be cut across, allowing the escape of cancer-cells which may infect the tissues with which they come in contact, and so lead to an early recurrence.

The growth may also spread by direct continuity along adhesions which have resulted from localised inflammation of septic origin. These facts emphasize the importance of wide removal of the disease whenever excision is performed.

The lymphatic vessels and glands of the dome of the stomach rarely are infiltrated. This portion of the stomach, for which Sir Berkeley Moynihan has suggested the term " isolated area," usually may be left with safety.

(ii) By Contiguity. - Spread of the disease by contiguity may occur in two ways $(a)$ by implantation on a contiguous surface, or $(b)$ by invasion of a neighbouring structure along adhesions.

(iii) By Dissemination.-Dissemination occurs through the lymphatic vessels. The liver is the organ affected most frequently (36 per cent. of the cases in my series).

The cases most suitable for radical treatment are those in which the growth is limited to the pyloric end of the stomach.

When the growth has invaded the duodenum, or has spread by continuity to the gastro-hepatic and gastro-colic glands, complete removal of the disease is practicable in 
many instances. When the growth has invaded surrounding structures by contiguity, e.g. the pancreas, complete removal is very difficult, often impracticable. When the growth has become disseminated to other organs, radical cure is impossible. Local removal can be only a palliative measure.

Unfortunately, in a large number of instances, when the patient first is seen by the surgeon, the growth is too advanced for radical cure.

It is a lamentable truth that many patients are allowed to die, who might have been relieved, if not cured, by a timely excision. From investigations I have made, I believe that in 20 per cent. of the fatal cases, there is no extension of the disease by contiguity, and no secondary deposits are discoverable at the post-mortem examination.

\section{THE SYMPTOMS OF GASTRIC CARCINOMA}

There is no sign or symptom pathognomonic of cancer of the stomach. In the early stages the symptoms are few and slight; in the later stages so plain, that he who runs may read.

Many of the so-called symptoms, such as ascites, jaundice, thrombosis, are late complications. When such are present, the opportunity for successful surgical treatment has gone for ever. To be of real benefit to the patient, the diagnosis must be made before these complications are present.

A discussion on these later so-called symptoms would serve no useful purpose. I propose, therefore, to deal with the symptoms of the earlier stages only, in which radical treatment may be possible.

The earliest symptoms of cancer of the stomach are; pain or discomfort after food, loss of appetite, failure of strength, anæmia, and loss of weight. 


\section{GASTRIC CARCINOMA AND SARCOMA 215}

Pain, or rather a feeling of discomfort and uneasiness after food, usually is the first symptom which attracts the patient's attention, insidious and scarcely perceptible at first. In some cases the onset is comparatively sudden, perhaps following some indiscretion in diet. At first its onset is determined by food, but as it increases in severity, it becomes more or less constant. It is accompanied by flatulence, and belching of gas. The pain is described as of a dull, sickening character, is referred to the epigastrium, and, as a rule, is less severe than the pain of gastric ulcer. Radiation of the pain is a late symptom, and means that the growth is invading the liver or pancreas. According to Dr. Fenwick, in 14 per cent. of the cases pain is either absent or extremely slight during the whole course of the malady.

Loss of appetite is gradual in its onset. The patient refuses food, not because it causes him pain, but because his appetite is gone. In many instances the patient evinces a marked dislike for meat.

Progressive loss of strength is an early symptom, sometimes the first to attract attention, and often is associated with impaired mental activity, and depression of spirits.

As a rule, even in the early stages of the disease, a slight but increasing degree of anæmia is present. In some cases a marked degree of anæmia is the earliest symptom of the disease. Such cases, in my experience, run a very rapid course, and are beyond the reach of surgery before a diagnosis is made. Fortunately, these form only a small proportion of the total number.

Even in the early stages of gastric carcinoma, almost invariably there is a gradual and progressive loss of weight. If, however, the patient be kept in bed, and be dieted carefully, in some instances the body weight may be maintained or even increased. This is a very important point, for I am convinced that a good many cases of cancer of the stomach 
are overlooked, because with rest in bed and dieting, the patient improves temporarily and puts on weight. It is not uncommon in hospital notes to find a record such as this: "The patient was admitted for supposed cancer of the stomach, but under medical treatment gained in weight, and was discharged much improved." Later, the patient comes under observation again, with obvious and inoperable gastric cancer.

I have observed a patient with cancer of the stomach put on as much as four pounds in weight in ten days. Such improvement is likely only in early cases, before the disease has spread beyond the limits of the pylorus-in other words, just those cases which would be benefited most by operation.

I wish, therefore, to lay particular emphasis on this point-namely, that gain in weight and temporary improvement under medical treatment and rest in bed, does not contra-indicate a diagnosis of cancer of the stomach.

The Presence of Blood in the Fæces.-The persistent presence in the fæces of blood, so small in amount that it is not recognisable macroscopically (occult blood), is very suggestive of gastric carcinoma. When the mucosa over a carcinomatous growth has become ulcerated, usually there is slight but persistent hæmorrhage, which can be recognised in the fæces by special tests. Ulcer may lead to the presence of occult blood, but in this disease the hæmorrhage is irregular and intermittent. It is only if the presence of blood be constant and persistent, that it is significant of cancer. For the test to be of value, the fæces must be examined after the patient has been on a blood-free diet for at least three days. The method of carrying out the test is described in the Appendix (p. 301).

A Palpable Tumour is not an early sign of cancer. In a few cases, however, the detection of a tumour is the first evidence of the disease, the other symptoms having passed 
GASTRIC CARCINOMA AND SARCOMA 217

unnoticed. This possibility emphasizes the importance of a careful and systematic examination of the abdomen in all cases of gastric trouble.

In palpating for a tumour the abdomen should be examined, not only after emptying the stomach, but after distending it with air. In some cases in which this is done, a hardening can be felt in the region of the pylorus, probably due to a spasm of the pylorus set up by the distension. When present this sign is an indication, not necessarily of cancer, but of the necessity for surgical treatment.

Our aim should be to establish a diagnosis, or at least to decide on the necessity of surgical treatment, before a tumour can be felt. Nevertheless a palpable tumour by no means contra-indicates the possibility of complete removal. One of my patients, who had a large tumour, not only palpable but visible, lived nearly four years after partial gastrectomy.

Hæmorrhage, as evidenced by hæmatemesis or by melæna, is a late, rather than an early symptom of gastric cancer.

Vomiting is an occasional symptom in the early stage of carcinoma of the stomach, but when the growth has produced pyloric stenosis, it is a constant and distressing feature. Usually the vomit is dark brown in colour, very acrid or offensive to the smell, and may contain blood in considerable quantity.

Although the existence of pyloric stenosis premises that the disease has progressed beyond the early stage, its presence does not negative the possibility of resection, because, when stenosis is marked, the condition of the patient is such, that a diagnosis of the necessity for surgical treatment is made at an earlier stage than when there is no obstruction at the pylorus.

In Carcinoma of the Cardiac End of the Stomach, the early symptoms are very definite. Usually, difficulty in 
deglutition is one of the earliest signs. The patient notices that he cannot swallow solid substances with ease, or as it is expressed by the patient, "the food sticks on its way into the stomach."

\section{Examination of the Gastric Contents}

When interpreted with the clinical history of the case, gastric analysis is of the greatest help, often the chief aid in assisting us to come to a conclusion as to the necessity for surgical treatment.

The three most important points are :

1. Evidence of Impairment of the Motor Functions of the Stomach.

2. Alteration of the Amount of Chlorides in the Gastric Juice.

3. Impaired Digestion of Albumen.

\section{Evidence of Impairment of the Motor Functions of the Stomach}

In 65 per cent. of the cases of gastric carcinoma, the disease begins at, or near, the pylorus. It follows, therefore, that one of the earliest signs of cancer of the stomach, is impairment of its motility. It is rare, even in the earliest cases, to find the stomach empty in the early morning. On passing a stomach-tube a varying amount of darkish fluid is withdrawn. Sometimes the quantity withdrawn amounts to 200 c.c. or 300 c.c., and has a very offensive smell. In the early morning contents of the stomach, free hydrochloric acid occasionally is present, in contrast to the usual absence of free hydrochloric acid in the gastric contents after a test-meal.

The quantity, moreover, of the gastric contents, withdrawn one hour after the test-meal has been given, usually is considerably above the average; the toast is ill-digested, the fluid contains a large amount of mucus, and consequently 
GASTRIC CARCINOMA AND SARCOMA 219

is so thick and ropy that the gastric contents are difficult to pour. Often it is possible to diagnose malignancy simply by inspection of the contents drawn off after a test-meal.

As a rule there is a considerable increase in the amount of the volatile acids, which vary from 5 per cent. to 20 per cent.

\section{The Alteration in the Amount of Chlorides}

(a) Diminution of the Total Chlorides.-The amount of total chlorides found by analysis after a test-meal, invariably is less, and usually considerably less, than normal. In my experience the amount is never more than 0.300 , and in about half the cases is less than $0 \cdot 200$.

(b) Absence of Free Hydrochloric Acid.-As a rule free hydrochloric is absent from the stomach contents in gastric carcinoma. For long this sign was regarded as pathognomonic of cancer. Now we know that this is not the case. Free hydrochloric acid often is absent in cases which are not malignant. Nevertheless, absence of free hydrochloric acid is the rule, presence of free hydrochloric acid the rare exception. I believe that when free hydrochloric acid is present, the growth is more likely to be situated at the cardiac end of the stomach.

(c) Diminution of the Protein Hydrochloric Acid.-A marked diminution of the protein hydrochloric acid, in my experience, is a very early symptom of gastric cancer. It is true that in chronic gastritis, due to causes other than malignant disease, there is marked diminution of the protein hydrochloric acid, but in estimating the value of this sign, we must consider its importance in relation to the clinical history. Chronic gastritis, secondary to cancer, progresses much more rapidly than gastritis due to other causes.

The relation of the degree of diminution of the protein hydrochloric acid to the duration of the patient's symptoms, 
must be considered. In other words, marked impairment of gastric motility, or marked diminution of protein hydrochloric acid, in a patient over middle age, raises a suspicion of malignancy, and the shorter the history and the greater the degree of gastritis, as shown by analysis, the greater the probability of cancer. Let me illustrate this by giving the analysis of the gastric contents of two patients, neither of whom had any palpable tumour.

No. 1

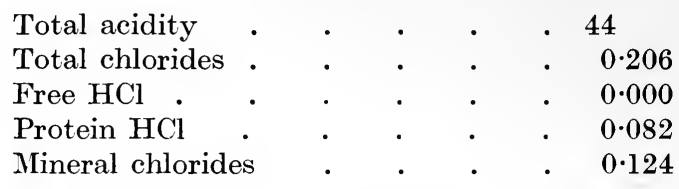

The above analysis was made from the gastric contents of a man who had suffered from gastric symptoms for a year. The chemical findings are those of a chronic gastritis, with very marked diminution of the protein hydrochloric acid. This might be due to inflammatory diseases or cancer. The comparatively long history, and at the same time the fair condition of the patient, and the absence of any degree of anæmia, were against malignant disease. Cancer of such standing should have resulted in a much lower total acidity, and in a more marked diminution of the protein hydrochloric acid. At the operation a gumma of the liver was found which had become adherent to, and implicated the pylorus.

No. 2

\begin{tabular}{|c|c|c|c|c|c|}
\hline Total acidity & - & & & & 29 \\
\hline Total chlorides & . & & & & $0 \cdot 183$ \\
\hline Free $\mathrm{HCl}$ & . & • & • & • & $0 \cdot 000$ \\
\hline Protein $\mathrm{HCl}$ & • & • & • & - & $0 \cdot 070$ \\
\hline Mineral chloride & & • & 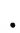 & ․ & $0 \cdot 113$ \\
\hline
\end{tabular}

This analysis was from the gastric contents of a woman who had suffered for four months from symptoms referable to the stomach. 
In this case the degree of gastritis was much greater than in the first case, and yet the history was much shorter. This, coupled with the fact that the patient evidently had lost flesh, and was slightly anæmic, led to a diagnosis of cancer, which was confirmed by operation, and the growth successfully removed.

I have not, as yet, met with a case in which the protein hydrochloric acid was about $0 \cdot 100$ or less, in which there was not some condition requiring surgical treatment.

(d) Increase of Mineral Chlorides.-The mineral chlorides as a rule, are increased, the amount being generally more than $0 \cdot 100$. Seeing that the amount of the total chlorides is diminished, it is probable that the increase of the mineral chlorides is due to neutralisation of free hydrochloric acid by an alkaline fluid secreted by the growth.

Usually the total acidity of the gastric contents is low, varying from fifteen to forty.

\section{Impaired Digestion of Albumen}

In gastric carcinoma the digestion of albumen, as tested by the carmine method, or by Hammerschlag's method, invariably is impaired. This impairment is not pathognomonic of carcinoma, but taken in conjunction with the history and other clinical signs, is a valuable adjunct to our means of diagnosis.

Other important points which can be ascertained by examination of the gastric contents are :

\section{The Presence of Unusual Substances in the Gastric Contents}

(a) Lactic Acid.-For lactic acid to be present in the stomach in excessive amount, four conditions are necessary, namely, gastric stagnation, absence or great diminution of 
the secretion of hydrochloric acid, impaired absorption, and reduced albumen-digesting power.

The presence of excess of lactic acid is not pathognomonic, but it is a valuable confirmatory sign, present, according to Dr. Fenwick, in 91 per cent. of all cases of cancer of the stomach. In my experience, it is not present in excess in early cases. The sign is of value only if the acid be present after a Boas' test-meal (see p. 10).

(b) The Oppler-Boas Bacillus.-Both Riegel and Hemmeter regard the presence of the long, non-motile OpplerBoas bacillus of considerable importance. Hemmeter and Adler found the organism present in 52 out of 55 cases of gastric carcinoma examined, while in 18 cases of gastric ulcer it was absent. According to Kauffman and Schlesinger the presence of a large number of bacilli is an indication of carcinoma.

(c) The Bacillus Filiformis.-According to Dr. Ehret the existence of cancer can be affirmed when masses of this long, slender, thread-like bacillus are found in the contents of the fasting stomach, provided that the contents of the fasting stomach are only moderate in quantity.

(d) Fragments of Growth.-The presence of fragments of growth in the gastric contents necessarily is diagnostic of cancer, but the presence of such fragments implies that the disease is advanced, and so is not of much value for the purpose of early diagnosis.

(e) Cancer Cells.-Hemmeter attaches considerable importance to the examination for cancer cells; he feeds the patient per rectum for forty-eight hours, and then washes out the stomach with saline solution. He uses a stomach-tube with a sharp eye which scrapes away minute portions of the gastric mucosa. The washings are centrifugalised and then examined microscopically. Cells in a state of atypical mitosis may be found. Personally, I must 
GASTRIC CARCINOMA AND SARCOMA 223

confess that I have not found any of these last four signs of much value in the early diagnosis of gastric carcinoma.

\section{The Examination of the Urine in Gastric Carcinoma}

1. Excretion of Uric Acid.-It is possible that the examination of the uric acid excretion may prove of value in the diagnosis of gastric carcinoma. In a healthy individual on a milk diet the amount of uric acid excreted varies from 0.1 to 0.3 grammes. In carcinoma, even on a milk diet, there is a considerable increase in the amount excreted; as much as 0.8 or even 1.0 gramme may be found in the urine collected during the twenty-four hours. So far as my observation goes, this is not so in gastric ulcer or in chronic gastritis, but whether this increase obtains in other conditions, as yet, I have not been able to determine.

2. Decrease in Phosphates.-Miss Elsie M. Royle has done some suggestive work in connection with urine in malignant disease. Miss Royle concludes as follows :

"1. The urine of a patient suffering from cancer is usually richer in uric acid than the urine of healthy persons under similar conditions.

"2. The output of phosphates in the urine is, in the majority of cases of carcinoma, less than is the case with healthy individuals.

" 3 . The ratio of phosphates to uric acid is almost invariably reduced in malignant disease below that found in health, any ratio below four being suggestive, especially if it remains low on several occasions. Any ratio below three is almost diagnostic of malignant disease, or of a blood disease-unless, indeed, further work proves that the same holds good for other conditions." 


\section{THE DIAGNOSIS OF GASTRIC CARCINOMA}

It is, I believe, to chemical pathology that we must look for the solution of the problem-How to diagnose gastric carcinoma in its early stage. At present examination of the gastric contents is our chief help, but it is not conclusive.

Dr. John B. Deaver, in urging earlier diagnosis of cancer of the stomach, says that the greatest source of delay is the test-meal, and that prolonged and useless laboratory investigation is responsible for the hopeless condition in which so many of the patients present themselves to the surgeon. I do not think that this is a fair way of stating the case. There is no need for prolonged investigation. No examination which is likely to prove of value need occupy more than a few days. It is the delay in giving the test-meal, not the delay due to the giving of the test-meal, which is responsible for so many inoperable cases. I am convinced that with routine use of the stomach-tube for diagnostic purposes in all cases of persisting indigestion, the number of inoperable cases of gastric cancer would be diminished greatly, the operation mortality reduced, and the number of cures proportionately increased.

Apart from finding fragments of the growth, or definite cancer cells in the stomach washings, our only certain means of diagnosis lies in abdominal exploration. The practical question then is, Under what circumstances is this justifiable? Are we to open the abdomen on suspicion, on probability, or only on certainty of the diagnosis? If we wait for certainty, in most cases we have allowed the opportunity for radical treatment to pass.

Are we to Open the Abdomen on Suspicion?-There must be suspicion of gastric carcinoma when a patient past middle life complains of persistent stomach trouble, with an onset, more or less abrupt, and without apparent cause. If, in spite of two or three weeks' treatment, improvement do not 
occur; if we find progressive diminution of the gastric - acidity and digestive power, or any other of the special signs to which I have alluded, suspicion becomes probability, and exploration should be delayed no longer. There is no doubt that many cases of gastric carcinoma are treated by medical means for long periods, in the hope that they may prove innocent. Such hope rarely is justified. In most of these cases, the condition found is such, that even if it be inflammatory and not malignant, relief can be obtained only by surgical means.

How many cases of pyloric stenosis, both innocent and malignant, are treated for many weeks by gastric lavage, and yet the futility of such treatment, as a curative measure, is well known! In an elderly person, the probability that even a slight degree of gastric stasis is due to carcinoma, is so great, that its persistence, in spite of careful dieting, renders exploration justifiable. When once we are satisfied as to the probability of cancer, the risk of abdominal exploration is less than the risk of delay. We must not shut our eyes to the axiom, that opening the abdomen is attended with risk, but the risk is reduced to a minimum, if we are content to limit our exploration.

If the disease be in a situation which permits of radical treatment, it is unlikely to be overlooked. The probability is, that a growth which cannot be palpated readily at an exploratory operation is inoperable, and its non-discovery of little practical importance. Some years ago I opened the abdomen of a man whom I supposed to be suffering from gastric carcinoma. I could find no growth. Two months later enlarged glands were noticed above the left clavicle. Some six months later he died from a carcinoma at the junction of the cesophagus with the stomach. Such cases are no argument against the value of abdominal exploration, but are an argument for tempering our zeal with discretion, 
and limiting both the extent and duration of our exploration. No one is more averse than I am from performing an " exploratory operation." At the same time, I think that the really reckless surgeon is not he who explores, after considering carefully the clinical history, and the results of gastric analysis, but he who adopts the policy of "wait and see," until the possibility of performing a radical operation is gone for ever.

There are occasions on which the symptoms, while uncertain, yet are so suggestive that exploration should not be postponed.

The information gained by exploration is not always conclusive. Many cases are on record in which, at the operation, the disease was considered to be malignant, but has subsequently proved to be inflammatory. Fortunately, in such cases the situation and relations of the growth are such that partial gastrectomy, as a rule, is out of the question.

With increasing experience, I believe that in the great majority of cases it is possible to distinguish inflammation from malignant disease, but it must be admitted, that in a few, differentiation is impossible.

To sum up : exploration is indicated as soon as there is a probability of gastric carcinoma. Probability is established when a patient in the middle period of life, suffers from gastric trouble which is progressive, unrelieved by treatment, and accompanied by any two of the following signs :

1. Absence of free hydrochloric acid, and impaired digestive power.

2. Marked diminution of the protein hydrochloric acid.

3. Constant presence of blood in the fæces.

4. Gastric stasis and impaired motility.

5. Abnormal excretion of uric acid.

I have not said anything about the gastroscope as an aid to diagnosis, because, although it is an exceedingly ingenious and interesting instrument scientifically, I am doubtful 
whether it will be of service in the diagnosis of cancer. Considering how difficult sometimes it is to decide whether a swelling be inflammatory or malignant, even when we can feel and see it, I do not think we can expect much help from an instrument which aids us by sight alone.

\section{Differential Diagnosis}

The following are the principal conditions which may give rise to difficulty in diagnosis: ulcer and its consequences, gall stones, severe anæmia, syphilis of the stomach, tubercle, and Bright's disease.

1. Ulcer and its Consequences.-When an extensive ulcer is present the symptoms may simulate closely those of cancer. There is emaciation, hæmorrhage, and consequent anæmia, absence of free hydrochloric acid, and impairment of the gastric motility. In the case of an ulcer, however, the percentage of the protein hydrochloric acid rarely is as low as in carcinoma. In cancer the loss of appetite is an early symptom, while the appetite of a patient with an ulcer remains good until a late stage, but he does not eat for fear of the pain caused by food. In the case of ulcer, the loss of flesh, and the failure of strength, are in proportion to the duration of the disease, and to the extent of abstinence from food. When cancer is present the loss of strength is more marked, progresses more rapidly, and is greater than can be accounted for by the diminished amount of food taken. When an ulcer of the duodenum has resulted in much contraction of the pylorus, or a gastric ulcer has produced an hour-glass stomach, the emaciation is so pronounced that a suspicion of malignancy is raised. The washing-out test (see p. 196), and the results of gastric analysis, usually will settle the diagnosis, although it must be remembered that occasionally malignant disease of the body of the stomach causes hour-glass contraction. 
The most difficult cases of carcinoma to diagnose are those in which there is a history of stomach trouble extending over many years. In such, as a rule, careful crossexamination will elicit the fact that there has been an exacerbation of the symptoms, which lately have become continuous, so that the patient no longer experiences the temporary remissions which occurred in the past.

It is, no doubt, as I have said already, difficult to distinguish gastric carcinoma from inflammatory disease, but there is no difficulty in deciding in which cases surgical treatment is indicated. The clinician may be forgiven if he fails to diagnose cancer, but failure to recognise the necessity for surgical treatment is less excusable.

2. Gall Stones.-Very occasionally the symptoms in the late stages of gall-bladder disease resemble those of gastric cancer. If we can obtain an early history of sudden, severe, irregular attacks of epigastric pain having no relation to food, we may be able to eliminate cancer from our choice of diagnoses. Gastric analysis, too, is helpful, as, although exceptionally, free hydrochloric acid may be diminished or absent in cholelithiasis, there is little or no marked diminution of the protein hydrochloric acid, and the total acidity of the gastric contents is not as low as in carcinoma.

3. Anæmia.-Dyspeptic symptoms and loss of appetite may accompany anæmia, and suggest malignant disease, a suggestion which may be supported by the gastric findings. In anæmia there is pallor, and a waxy appearance of the skin, and often puffiness of the face. Examination of the blood reveals poikilocytosis, low hæmoglobin, and a highcolour index. The spleen may be enlarged. In cancer the percentage of hæmoglobin is either normal, or above normal, and the colour index usually low.

4. Syphilis.-Syphilis of the liver or stomach in the early 
stages may simulate ulcer, and in the later stages, when cachexia is marked, may suggest cancer. On analysing the gastric contents the percentage of the protein hydrochloric acid often is found to be low, but the total acidity is higher than is the case in malignant disease. The patient's general condition, moreover, is much better than would be the case in gastric carcinoma of similar duration. The following case illustrates very well how the two conditions may be differentiated:

A man, aged thirty-three, under the care of my colleague, Dr. S. Fenwick, had suffered from pain, and a feeling of fullness after meals, for about a year. Some months before admission he began to suffer from vomiting, the vomit often being greenish in colour and containing altered food. The patient had lost flesh considerably. There was a history of syphilis. On admission there was a movable, insensitive swelling about the size of a walnut, above and to the right of the umbilicus. The swelling moved with respiration, and could be distinguished from the liver. The stomach was dilated considerably. The patient was very thin and sallow, but otherwise healthy and in good condition.

\section{Gastric Analysis}

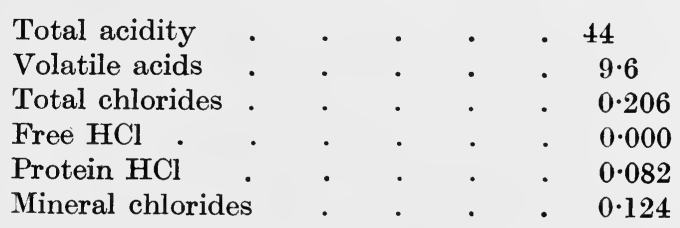

The chemical findings were those of chronic gastritis, suggesting malignant disease.

The absence of marked anæmia, and the good condition of the patient, considering the long history, were against malignant disease. The diagnosis made was " inflammatory pyloric stenosis." At the operation I found a hard, nodular mass under the surface of the right lobe of the liver, extending backwards for some 
distance. On incising this, some gummatous material escaped, suggesting a gumma. A portion frozen and microscoped at once showed no evidences of malignant disease. The cavity was drained. The duodenum was adherent to the under surface of the mass, and was obstructed by adhesions. Posterior gastrojejunostomy was performed, and the patient was completely relieved, and has, since his operation five years ago, remained in perfect health.

5. Tuberculosis.-Tuberculous ulceration of the intestine may, in its later stages, suggest gastric carcinoma. This is the case especially when the ulceration results in stricture of the intestine. Emaciation, pain after food, loss of appetite, and vomiting, make a clinical picture closely resembling cancer. In addition the findings from analysis of the gastric contents are those of malignant disease, except that the motility of the stomach is not impaired to the same degree as in cancer. The slow onset of the disease, the hectic, irregular temperature, suggest tubercle rather than cancer, and by the time that the disease is sufficiently advanced to resemble cancer, there are usually lung complications, and tubercle bacilli in the sputum.

A man, aged thirty-four years, came under my care, who for six years had suffered from pain in the stomach, coming on from one to three hours after food. The pain was often accompanied by vomiting. Latterly he had been much troubled with flatulence and constipation. The patient was extremely emaciated, and his stomach was greatly dilated.

\section{Gastric Analysis}

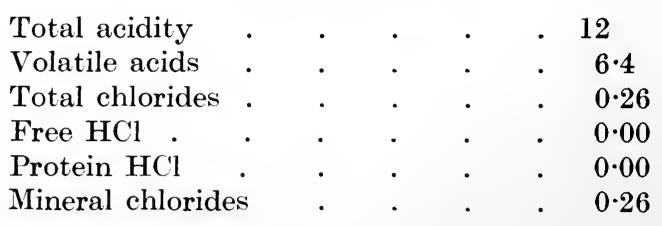

The gastric analysis suggested cancer, but the absence of 
impaired motility of the stomach, the irregular temperature, and the long history were against malignant disease. It was thought that his condition was due to tuberculous peritonitis. On opening the abdomen I found very enlarged mesenteric glands, and two cicatrical strictures of the small intestine, evidently the result of contraction of two tuberculous ulcers. The strictures were short-circuited by a lateral anastomosis, and the patient was relieved of his pain and vomiting, but died about nine months later from tubercle of the lungs.

6. Chronic Nephritis.-Chronic nephritis may be accompanied by anæmia, vomiting, loss of appetite, and wasting to such a degree that cancer of the stomach is suggested. The history of increased frequency of micturition, the condition of the blood-vessels, and the examination of the urine usually will reveal the true nature of the case.

\section{SARCOMA OF THE STOMACH}

Frequency.-Primary sarcoma of the stomach is a rare disease, though probably less uncommon than is supposed to be the case. Some specimens recorded as carcinomata have been found later really to be sarcomata. Drs. Perry and Shaw, on re-examining fifty museum specimens of gastric carcinoma, found that four were sarcomata. Dr. Soltau Fenwick estimates that from 5 to 8 per cent. of all primary neoplasms of the stomach are sarcomata. I think this is over-estimating the frequency of sarcomata, and I believe 4 per cent. to be nearer the correct proportion.

An important feature of gastric sarcoma is its occurrence in the early years of life. A number of cases in children have been recorded, one by Dr. H. Thursfield in a child aged four years.

Gastric sarcoma may grow outwards, and so present itself as a tumour on the external surface of the stomach. Such tumours are termed "exogastric" sarcomata. 
Pathology.-All varieties of sarcoma have been found in the stomach. Those found most usually are round-celled sarcomata, originating in the sub-mucous tissue, and spindlecelled or fibro-sarcomata, occurring generally as circumscribed, rounded tumours of the gastric wall.

Spindle-celled sarcomata may attain a large size. Cartwell has reported an instance, in which a fibro-sarcoma of the stomach weighed twelve pounds.

Less commonly the two following types of sarcoma occur : lympho-sarcoma, commencing in the lymphatic nodules of the sub-serous coat, and myo-sarcoma, having its startingpoint in the muscular wall. Unlike carcinoma, sarcoma originates usually in the body of the stomach. The roundcelled variety is most malignant, and metastases are found most commonly in the liver.

Symptoms.-The symptoms of gastric sarcoma are similar to those of carcinoma. The general symptoms are most marked in the round-celled type, in which early and progressive anæmia is very common. In some instances, especially in the cases of slow-growing fibro-sarcomata, the symptoms may pass almost unnoticed. In one case which I saw, the first symptom which attracted attention was a severe hæmorrhage which proved fatal within a few hours.

Dr. E. von Graff has reported the case of a young woman, aged twenty-seven, who had suffered from occasional attacks of weakness. After two years she noticed that her waist had grown larger, and found that she had lost fifteen pounds in weight. At the end of three years a large tumour had made itself apparent in the lower abdomen, reaching to the umbilicus. There was no anæmia, dyspepsia, or constipation. The tumour grew from a small segment of the greater curvature, and was removed easily by resecting a small portion of the stomach with it.

As the pylorus is affected less frequently in gastric 


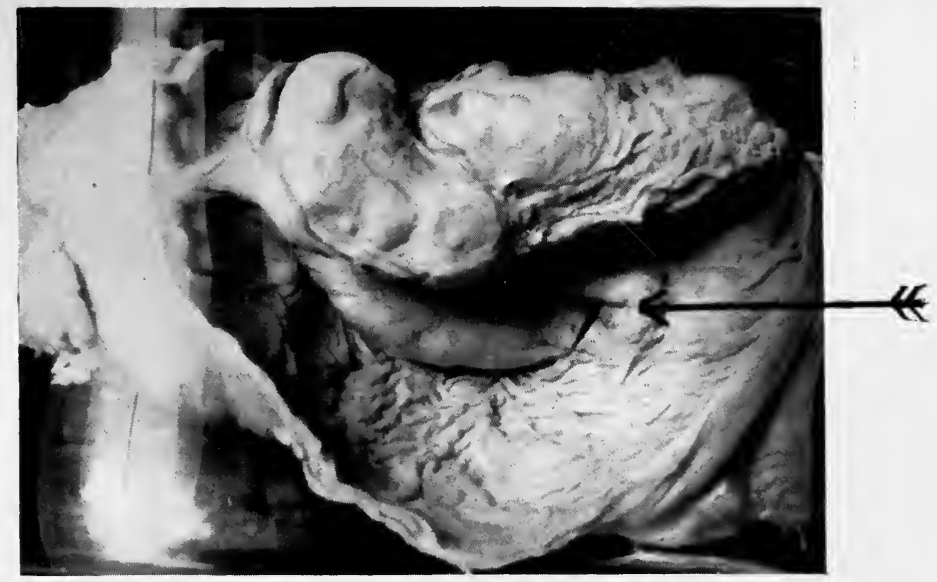

FIG. 66.-A sarcoma of the stomach, from a woman aged 22 , who died with symptoms of perforation. The arrow points to the seat of hrmorrhage which was the cause of death.

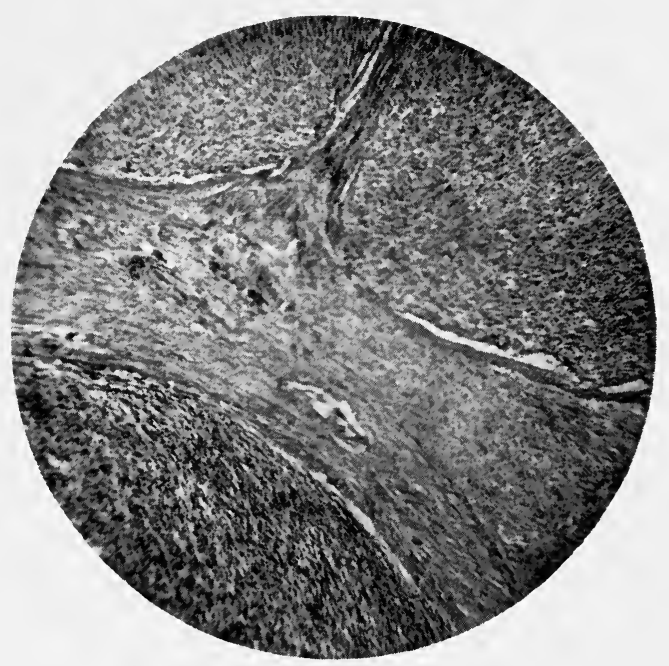

FIG. 67.-A microphotograph of the same case. 
sarcoma than in gastric carcinoma, vomiting is less common. In fibro-sarcomata and myo-sarcomata, a palpable tumour almost always is present, while in round-celled sarcoma a tumour is felt less commonly.

The differential diagnosis of sarcoma from carcinoma in most cases is impossible. The younger the patient the greater is the probability. of sarcoma, and early anæmia and pyrexia are in favour of sarcoma. As to the findings of gastric analysis in gastric sarcoma, I have had no experience.

\section{REFERENCES-CARCINOMA OF THE STOMACH}

\section{Pathology}

Acher, Deutsche Archiv f. Klin. Med., x. p. 173.

Billroth, Virchow's Archiv, 1861, xxi. p. 423.

Bristow, Trans. Path. Soc., xxii. p. 137.

Cayley, Trans. Path. Soc., xviii. p. 90.

Cohnheim, Archiv f. Path. und Anat., 1867, p. 142.

Cruveilhier, Path. Anat., x.

Cuneo, Thèse de Paris, 1900.

Ebstein, Volkmann's Sammlung Klin. Vorträge, 87, p. 21.

Ely, Amer. Jour. Med. Sc., 1890, p. 584.

Féréol, Bull. Soc. Anat., 1857, p. 459.

Gerota, Anatomische Anzeiger, Bd. xii., 1896.

Haeberlin, Archiv f. Klin. Med., 1889, p. 46.

His, Die Anat. Nomenclature, Verlag v. Veit und Comp., 1895.

Hofmann, Zeit. f. Klin. Med., 1897, p. 460.

V. Jez, Wien. Med. Woch., 1898, p. 633.

Kaufmann, Virchow's Archiv, lxxv. p. 317.

Lengemann, Arch. f. Klin. Chir., Bd. Ixviii.

MacCarty, Surgery, Gyncecology, and Obstetrics, May 1910, x.

Mathieu, Archiv gén. de Méd., 1889, p. 402.

Most, Verhand. d. Deutsch. Gesselsch. f. Chir., 28 Kongr., 1899, ii.

Ott, Diss. Zurich., 1867.

Orth, Lehrbuch der Spez. Path. Anat., 1887.

Perry and Shaw, Guy's Hospital Reports, xlviii. p. 137.

Petrick, Deut. Zeits. f. Chir., 1891, Bd. xxxii. p. 530.

Renner, Mitteil a. d. Grenzgebieten d. Med. und Chir., xiii.

Ribbert, Virchow's Archiv, 135, p. 433.

Riegel, Zeit. f. Klin. Med., 36.

Rindfleisch, Trait. d'hisol. Path. Trad. franc., 1888. 
Rosenheim, Zeit.f. Klin. Med., 1891.

Sappey, Anat. Physiol. Path. des Vaisseaux Lymph. Paris, 1874.

Zender, Virchow's Archiv, 1890, cxix. p. 261.

\section{Diagnosis}

Ascoli and Izar, Munich. Med. Woch., 1910, No. 41.

Boas, Speciel-Diagnostik und Therapie der Magen Krankheiten, und Deutsche Med. Woch., 1892, p. 370.

Bird, Golding, London Med. Gaz., 1842, p. 391.

Capps, Boston Med. and Surg. Jour., 1897, p. 498.

Chiaruttini, Gaz. d. osp. Milan, 1897, p. 613.

Ehret, E., La Semaine Médicale, Mar. 6, 1901.

Einhorn, Diseases of the Stomach.

Elsberg, Amer. Jour. Med. Sciences, Feb. 1910.

Ewald, Diseases of the Stomach.

Graham, Chris., North-Western Lancet, April 1, 1910.

Hammerschlag, Archiv f. Verdauungs Krankheit., 1896, i.

Hampeln, Zeit. f. Klin. Med., viii. p. 232.

Hartmann and Silhol, Revue de Chir., 1901, No. 2.

Hemmeter, The Lancet, 1901, ii. p. 829.

Hofmann, Zeit. f. Klin. Med., 1897, p. 460.

Lindner and Kuttner, Die Chirurgie des Magens, 1896.

Neubauer and Fischer, Deut. Archiv f. Klin. Med., 1909, xcvii. p. 499.

Osler and Macrae, Cancer of the Stomach, 1900.

Paterson, H. J., Brit. Med. Jour., 1910, ii. p. 953.

Reineboth, Deut. Archiv f. Klin. Med., lviii. p. 62.

Riegel, Zeit. f. Klin. Med., 36.

Royle, Elsie, Woman's Med. Jour., April 1911.

Sailer and Taylor, Internat. Med. Mag., 1897, p. 404.

Schiff, Centralblatt f. die Grenzgeb. der Med. u. Chir., 1898, xii.

Schlesinger and Kaufmann, Wieser Klin. Runds., 1895, No. 15.

Schüle, Munich. Med. Woch., 1897, p. 737.

Stienon, Jour. de Méd. de Brux., 1888.

Weber, Jour. Amer. Med. Assoc., 1896, p. 8.

\section{Sarcoma of the Stomach}

Brooks, Med. News, 1898, p. 617.

Cartwell, Annals of Surgery, 1899, ii. p. 596.

Cayley, Trans. Path. Soc., 1869, xx.

Dock, Jour. Amer. Med. Assoc., 1900, xxxv. p. 156.

Ewald, Klinik. d. Verdauungs Krankheit., ii. p. 354.

Fenwick, Cancer of the Stomach, 1902, p. 271, and The Lancet, 1901, i. p. 463.

Feuerer, Deutsche Zeit. f. Chir., 1912, cxvi.

Von Graff, Wiener. Klin. Woch., 1912, xxv. p. 991. 


\section{GASTRIC CARCINOMA AND SARCOMA 235}

Hadden, Trans. Path. Soc., 1885, xxxvii. p. 234.

Handford, Trans. Path. Soc., 1888, xl. p. 89.

Hartley, Annals of Surgery, 1896, xxiii. p. 609.

Kundrat, Wien. Klin. Woch., 1893, p. 12.

Legg, Trans. Path. Soc., xxv. p. 121.

Lofaro, Archiv Génér. de Chir., Aug. 1909.

Lowe, Brit. Med. Jour., 1886, ii. p. 1033.

Perry and Shaw, Guy's Hosp. Reports, 1892, p. 48.

Riegel, Die Erkran Rungen des Magens, ii. p. 874.

Schlesinger, Zeitschr. f. Klin. Med., 1897, p. 179.

Thursfield, H., Trans. Path. Soc., 1901, lii. Part 1. 


\section{CHAPTER XVII}

\section{THE TREATMENT OF GASTRIC CARCINOMA AND SARCOMA}

Probably it is hardly realised yet, that although the stomach is more frequently affected with cancer than any other organ in the body, partial gastrectomy is one of the uncommon operations of surgery. This is not as it should be, for gastric carcinoma is markedly a local disease, and can be removed with favourable results, both immediate and remote, whereas no other form of treatment can hold out the slightest prospect of any lasting benefit. The good results which can be obtained in rectal or mammary cancer are well known. There is no reason why the results of surgical intervention for gastric cancer should not be as good, or indeed better, for gastric carcinoma, in its early stage, lends itself to wide removal even more favourably than cancer of the breast or rectum.

Cancer of the Pylorus.-Pyloric cancer should be treated by partial gastrectomy, whenever this is possible (p. 42).

As I pointed out in the previous chapter, the margin of induration in the stomach wall corresponds to the limit of the growth in the mucous membrane. Invariably the disease extends in the submucosa, beyond the limits of the disease in the other coats of the stomach. In partial gastrectomy, therefore, the line of section of the stomach must be made well beyond the indurated margin. In every case, at least one inch of the duodenum, the whole of the lesser curvature, together with the gastro-hepatic glands, must be removed. As to the site of section along the greater curvature, there is some difference of opinion; some surgeons 
TREATMENT OF CARCINOMA AND SARCOMA 237

make the section about the junction of the pyloric with the middle third, others make the section considerably further to the left. Personally, I remove at least half of the greater curvature in early cases, and in more advanced cases I make the division close up to the isolated area, thus removing two-thirds of the stomach, together with the gastro-colic glands lying along the greater curvature.

If the glands at the lower end of the œsophagus, or round the cœliac axis, be infected with carcinoma, a radical operation is impossible, but the surgeon must not assume, because these glands are enlarged, that the enlargement is due to infiltration with carcinoma. In these cases, therefore, it is my practice to remove one of the suspected glands for microscopical examination, then to perform gastro-jejunostomy, the resection being postponed until a later date. If the enlargement of the glands prove to be inflammatory, the resection can be performed three weeks later.

As I have pointed out in the previous chapter, the glands along the greater curvature communicate with the glands lying in the gastro-colic omentum, along the upper border of the transverse colon. In some cases these latter glands are enlarged. If the enlargement be due to carcinoma, then I am of opinion that the transverse colon should be resected. I am convinced, from an examination of many specimens, that recurrence occurs, not infrequently, either in, or close to, the transverse colon, and I believe that such recurrence would be prevented, in some cases, by removal of the transverse colon with its lymphatic glands.

A patient upon whom I had performed partial gastrectomy returned to me nearly three years later, with a large mass in the upper abdomen which I took to be a recurrence. Two months later she was admitted into the London Temperance Hospital in a dying condition, with signs of general peritonitis. At the post-mortem examination general 
suppurative peritonitis was found, the result of perforation of a stercoral ulcer on the proximal side of a small, hard, ring-like stricture of the transverse colon due to a small carcinomatous growth. The large swelling felt during life consisted of a mass of matted omentum and intestine, evidently due to plastic peritonitis secondary to perforation of the stercoral ulcer. The secondary, like the primary growth, was a spheroidalcelled carcinoma. It could have been removed, so that had recognition of the true nature of the abdominal swelling been possible, the patient's life might have been saved. In this case, as in others I have seen, recurrence might have been prevented by excision of the colon. I think, therefore, the advisability of resection of the transverse colon must be considered, when the glands draining it are affected. This does not prolong the operation as much as might be expected, because, although the anastomosis takes extra time, less time is occupied in dealing with the gastro-colic omentum. At the same time, excision of the transverse colon does materially increase the risk of operation.

Personally, I am not in favour of very extensive operations as a routine treatment for malignant disease. I think that if our results are to be improved, it will be by earlier, not by more extensive, resections. In some cases, however, an extensive operation is necessary if the disease is to be removed at all. In an advanced case, the advisability of such an operation depends on the general condition of the patient. Severe anæmia is the chief contra-indication to partial gastrectomy. Such patients do not bear operation well, and usually metastatic deposits are present already. The presence of secondary growths in the pelvis is an absolute contra-indication to resection, therefore a pelvic examination should be made before deciding on surgical treatment. Patients with a very low blood-pressure are bad subjects for a severe operation. Extreme emaciation is not a bar to 


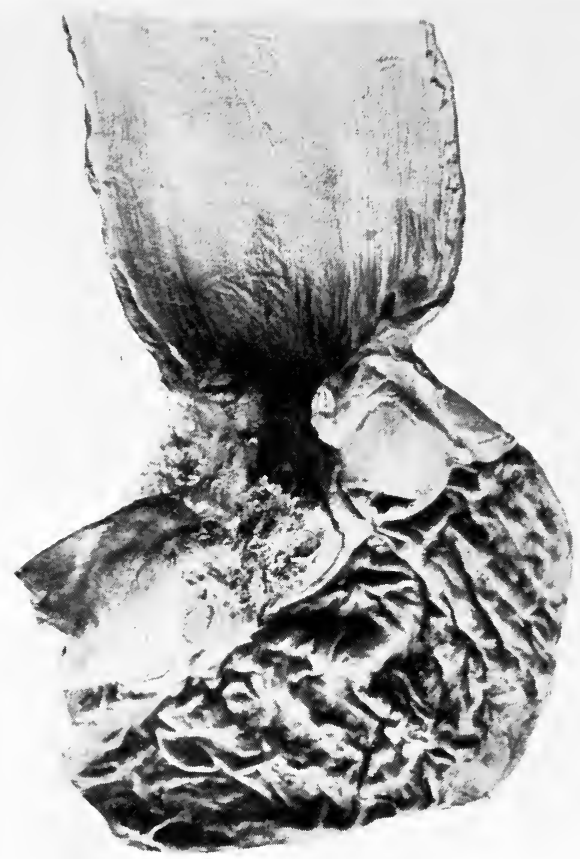

FIG. 68.-Carcinoma of the cardiac end of the stomach. The dilatation of the osophagus above the stricture is well seen. (Royal College of Surgeons' Museum. No. 2417.) 
a radical operation. The thinness of the patient facilitates operation, and as the condition of such patients usually is the result of lack of nourishment, they bear operation better than those who are being poisoned by the absorption of toxins from the growth. Much may be done to improve patients, with gastric carcinoma, for operation, by daily lavage, and by feeding them on mutton or beef essence and milk. In bad cases the period of preparation should be at least a week or ten days. The patient often will gain two to three pounds in weight, and will be in a much better position to undergo the operation.

Cancer of the Body of the Stomach.-In certain cases of cancer forming a ring-like growth at or near the middle of the stomach (cancerous hour-glass constriction), it may be possible to excise the growth and to unite the two separated portions of the stomach. I do not favour this plan. In the endeavour to preserve sufficient of the stomach to enable the junction to be effected without tension, the surgeon is likely to remove the disease insufficiently widely. In these cases I prefer to do a partial gastrectomy and gastro-jejunostomy in the same manner as for pyloric cancer.

Cancer of the Cardiac End of the Stomach.-Partial cardiac gastrectomy for cancer of the cardiac end of the stomach, as yet, has not been performed successfully on a human being. Mikulicz has operated once for this condition, but unsuccessfully. The objections to the operation are: first, the danger of injury to the pneumogastric nerves, and secondly, the difficulty of isolating the lower end of the œsophagus sufficiently freely to anastomose it to the stomach.

Krehl, however, has demonstrated that in dogs, injury of the nerves at the lower end of the œsophagus is unattended with any impairment of nutrition, and Levy demonstrated the possibility of resecting the cardiac end of the stomach in the dead body. 
To carry out such a resection, however, when a mass of malignant disease is present, is a very different matter. Mikulicz has written in optimistic vein as to the possibilities of the operation, but at present the radical treatment of malignant growth about the cardiac orifice is beyond the range of practical surgery.

\section{The Results of Gastrectomy for Malignant Disease}

A. Immediate.-In my Hunterian lectures in 1906 I collected a series of 436 cases of partial gastrectomy by various surgeons, with a death-rate of 28 per cent., and I pointed out that the operative results were steadily improving and that the mortality rate at that time was well under 20 per cent.

Since that time there has been a still further improvement. At the Rochester clinic during the period 1897 to 1909, partial gastrectomy was performed by Drs. W. J. and C. H. Mayo 266 times with 34 deaths, an average mortality of $12 \cdot 4$ per cent. During the year 1909 there were 46 operations with four deaths, an average mortality of 8.6 per cent. My own mortality rate is 14 per cent. I think we may conclude fairly, that at the present time the mortality rate is under 15 per cent., and that if patients are submitted to operation at an earlier stage, before they become exhausted by vomiting, and by poisoning from the ulcerated growth, the operative mortality need not exceed 5 per cent.

B. Remote Results.-The remote results of gastrectomy may be considered from two points of view ; first, as to the effect of removal of all, or part, of the stomach on the health of the patient; and, secondly, as to the freedom from recurrence of the disease.

While the value of a good stomach is undoubted, fortunately Nature is able to dispense with this organ, as most of its functions can be performed vicariously by other portions of the alimentary canal. The stomach performs four main 
functions. First, it acts as a reservoir, but after its complete removal the lower end of the cesophagus becomes dilated and serves this purpose, although to a limited extent. Secondly, the mechanical function of the stomach-namely, maceration - can be replaced by careful dieting. Thirdly, the secretory or chemical functions, the secretion of pepsin, hydrochloric acid, and rennin, can be replaced effectively by the intestine. Thus, the functions of the rennin can be performed by the pancreatic juice, while the antiseptic action of the hydrochloric acid, which prevents the formation of lactic acid, is carried on by the bile. Fourthly, the resorptive functions, by which peptones are absorbed, are relegated to the small intestine. Nature has thus duplicated three out of the four functions performed by the stomach, although the loss of the gastric secretion cannot be compensated altogether; " those who have lost it," says Lubarsch, " have one weapon less in the struggle for existence." Pachon and Carvalho have shown that dogs may gain in weight and remain in perfect health after removal of the entire stomach, while the elaborate observations made on Schlatter's patient, prove that the same holds good of human beings. These observations record that the absorption of albumin was unaffected, and that no putrefactive changes occurred in the intestines, which shows that the absence of the gastric juice does not lead to decomposition in the intestines.

Next as to the freedom from recurrence. In my previous book on gastric surgery I published the following table showing the after-results of total and partial gastrectomy :

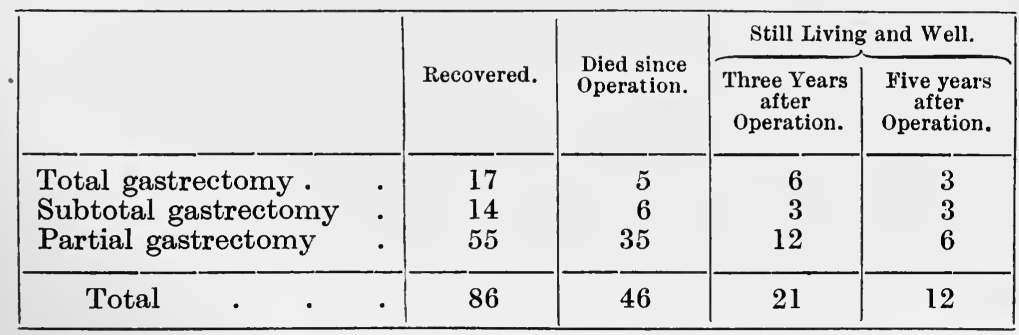


When recurrence ensues after partial gastrectomy, it does so usually within four years. Dr. W. J. Mayo has reported a case in which death from recurrence occurred after eight years, but this is exceptional. If a patient be free from recurrence after four years, there is a reasonable prospect of cure. Judged by this standard, 15 per cent. of the patients in this series who recovered from operation were cured. At the present time even more favourable results are being obtained. In a later report Dr. W. J. Mayo states that of the patients operated on more than three years ago, who recovered from operation, 29 per cent. have remained well. Personally I can point to one patient alive and well seven years after partial gastrectomy, and two over five years. With earlier diagnosis and earlier operation we shall obtain even better results, for there is no reason why, in cancer of the stomach, we should not reach the 50 per cent. of cures, which has been obtained by Dr. Halsted in the operative treatment of cancer of the breast.

Palliative Operations.-Opinions are divided as to the advisability of performing resection in cases in which the whole of the infected glands cannot be removed.

Under such circumstances, some surgeons are in favour of an incomplete or palliative resection for three reasons: first, because the mortality of resection is little greater than that of gastro-jejunostomy ; secondly, if the patient recover from the operation, life is more comfortable and more prolonged than after gastro-jejunostomy; thirdly, as Mikulicz points out, the patient is benefited greatly by the removal of the sloughing mass of growth.

Riese follows the teaching of Mikulicz, and advises extensive resections for carcinoma, even though the disease be widespread. He believes permanent cures may be hoped for, even when there is extensive glandular disease. Let us briefly consider the propositions here stated. It is 


\section{TREATMENT OF CARCINOMA AND SARCOMA 243}

quite true that the mortality of gastro-jejunostomy for gastric carcinoma is high. The cases in which it is performed necessarily are advanced cases. If, however, partial gastrectomy is to be adopted as the routine treatment in very advanced cases, I feel sure that the mortality would prove to be, not a little higher, but a great deal higher than that of gastro-jejunostomy. Whether life is prolonged appreciably by a resection which leaves disease behind, is open to doubt; at any rate the gain is not great, and if the increased mortality rate be taken into account, I question whether, on the balance, resection would show an increased gain in life as compared with gastro-jejunostomy. There is room for difference of opinion as to whether patients are more comfortable after a palliative resection. Doubtless the majority are, but many patients, after a gastro-jejunostomy, live in comfort almost to the last.

As to the statement, that the removal of the sloughing mass of growth greatly benefits the patient, I would reply that a sloughing mass is not present in all cases. The benefit derived from a palliative resection will depend on the type of the growth. With a hard, constricting, slow-growing carcinoma of the pylorus, the patient suffers from starvation more than from toxæmia; with a soft, fungating growth toxæmia is a prominent symptom. In the latter cases patients are undoubtedly much benefited by the local removal of the growth.

It may be argued, that as palliative resection of a cancerous ulcer of the tongue, the inoperable glands being left, is a very beneficial proceeding and is considered to be good surgery, so palliative gastrectomy should be performed for the same reason. There are, however, two important differences in the two cases. In cancer of the tongue, pain is a constant and distressing symptom. In gastric carcinoma, apart from obstruction, which can be relieved by 
gastro-jejunostomy, this is not so. The relief afforded by a palliative resection is much greater in lingual than in gastric carcinoma. Secondly, local removal of a portion of a cancerous tongue, the glands being left, is comparatively safe; partial gastrectomy in an advanced case is a very serious operation.

At this stage let me point out that wide resection may be performed for two totally distinct reasons. A wide resection may be done in an early case, in order to make certain of removing all the microscopic ramifications of the disease. On the other hand, a very extensive excision may be absolutely necessary in order to extirpate all the macroscopic disease. These two classes of gastrectomy should be distinguished clearly. Both the immediate and remote results are in marked contrast. A wide resection in an early stage of the disease is attended with a low mortality rate, and with good remote results. Extensive operations in advanced cases have a high death-rate, and the ultimate results are disappointing.

The advocates of palliative resection may argue, that even in an early case it is possible that, however wide the resection, some cancer-cells are left behind. Mr. Sampson Handley has shown, that carcinomatous cells permeate the lymphatic channels very widely from the primary growth. Fortunately, the clinical results of resection are vastly better than pathological teaching leads us to expect. We must, therefore, suppose that Nature, in some measure at least, is able to deal with the cancer-cells which are left behind. If this were not so, resection, even in an early case, would be a futile proceeding, if we accept as true all that pathologists tell us.

To what extent, and under what circumstances, Nature does this, we are in the dark. We have, however, no evidence that if infected glands be left behind, permanent cure can result. 
TREATMENT OF CARCINOMA AND SARCOMA 245

This being so, my view is, that if there be inoperable carcinomatous glands, it is not worth while to submit the patient to the risk of an extensive resection, the benefit of which cannot but be short-lived. Before deciding against resection in a given case, the surgeon must ascertain by microscopical examination whether the enlargement of the irremovable glands is due to cancer or to simple inflammatory infiltration.

Even in those advanced cases in which it may be possible to remove all the disease by means of a very extensive operation, the advisability of resection, I think, is doubtfuI.

In early cases I am an advocate of resections which are wide in comparison with the extent of the disease, but I am not so sure of the benefit resulting from resections which have to be extensive on account of the advanced stage of the disease. The surgeon ought not to have to consider this problem, and it would be seldom necessary to do so if the stomach-tube, and other available means of diagnosis, were resorted to in the routine of practice. Early diagnosis must be our aim, and it is by realising this ideal, and not by the performance of more elaborate and extensive operations in advanced cases, that the results of the surgical treatment of gastric carcinoma will be bettered.

In cases of pyloric cancer, with gastric stasis and food retention, too advanced for resection, a gastro-jejunostomy should be performed.

The increased nutrition, and relief from vomiting, greatly benefit the patient. The operation, however, should not be advised if there be ascites, or marked hepatic enlargement. Under such circumstances the risk of the operation is so great, and the prospect of prolongation of life so small, that it is better to treat the stenosis by daily gastric lavage. Unless there be food retention, I do not think that a shortcircuiting operation is of any benefit. 
In those cases in which the whole of the stomach is infiltrated with cancer, so that a gastro-jejunostomy is impracticable, the advisability of performing a jejunostomy may be considered. The advantages of this operation are very doubtful. It is not to be recommended except in a few exceptional cases, in which there is some urgent reason for making an attempt to prolong the patient's life for a few weeks. Under ordinary circumstances the benefit to be derived from it is so slight, that it is not worth doing.

In cancer of the cardiac end of the stomach, or of the œsophagus, gastrostomy is the only surgical procedure available. In my judgment, it should not be advised until the patient no longer is able to swallow sufficient nourishment to maintain his body weight. I do not agree with the teaching that gastrostomy should be performed as soon as the diagnosis has been made. If this practice be followed, it is almost certain that in some cases, the operation will precede the cancer. I have seen patients who have had a gastrostomy performed and who have refused to die, because they have been the victims, not of cancer, but of an erroneous diagnosis.

In hard cancerous strictures of the cesophagus the use of a Symonds' tube (Fig. 69) often is very valuable. This

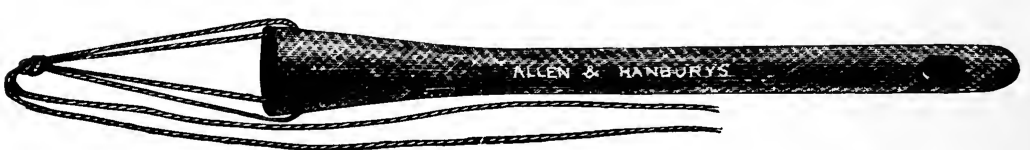

Fig. 69.-A Symonds' Tube.

instrument is less used than its merits deserve. Why, I do not know. If it be passed by the aid of a string guide as suggested by Dr. Plummer, it is as safe as gastrostomy, and in many cases is preferable. In the soft fungating growths its use is attended with greater risk, but even in these cases its use for a few days often will enable the surgeon to feed 


\section{TREATMENT OF CARCINOMA AND SARCOMA 247}

up a starved patient and bring him into a better condition for operation.

Patients with cardiac or esophageal cancer die more often from broncho-pneumonia and metastases than from starvation. In a few selected cases gastrostomy prolongs life and adds to the patient's comfort. The operation, therefore, has its uses, but the number of cases in which it is of marked benefit is limited.

The immediate operation mortality is very small, probably about 2 or 3 per cent.

\section{REFERENCES}

Czerny, Jour. de Chir., Oct. 1908.

Feuerer, G., Deut. Zeit. f. Chir., 1912, cxvi.

Groves, E. W. H., Brit. Med. Jour., Feb. 12, 1910.

Levy, Archiv f. Klin. Chir., 1898, lviii. p. 839.

Mayo, W. J., Jour. Amer. Med. Assoc., 1910, liv. p. 1608.

Moynihan, B. G. A., Abdominal Operations, 1906, p. 257.

Poncet, Gaz. des Hôpitaux, Mar. 25, 1909.

Riese, Deut. Med. Woch., April 23, 1908.

Tupolske, Philadelphia Med. News, Mar. 1893, p. 263. 


\section{CHAPTER XVIII \\ ULCUS CARCINOMATOSUM}

OF late years there has been considerable discussion as to the frequency with which cancer is grafted on simple ulcer of the stomach. Cruveilhier, in 1839, and Dittrich, in 1848, were the first to point out this possibility.

Zenker, in 1882, went further, and put forward the hypothesis that all cases of gastric carcinoma have their origin in simple ulcer. More recently Drs. W. J. Mayo and Christopher Graham have laid great stress on the origin of cancer in ulcer, and Drs. Wilson and MacCarty from the same clinic, have contributed a valuable paper dealing with the problem from a pathological standpoint. These writers state that they have been able to demonstrate a precancerous or non-malignant ulcer in 62 per cent. of their cases. Most observers, however, have placed the proportion at a much lower figure. Thus, according to Sonicksen, 14 per cent. of all cases of cancer of the stomach originate in ulcers; Lebert estimated the frequency at 9 per cent.; Rosenheim at 6 per cent.; Fenwick and Häberlin at 3 per cent.

The possibility that carcinoma may originate in an ulcer cannot be denied; however, it is very doubtful whether such an occurrence is as frequent as some authorities would have us believe. The problem is a very difficult one, and a dogmatic conclusion impossible, but the evidence available in favour of the view that simple ulcer is a frequent precursor of cancer is not convincing. This evidence is both clinical and pathological. 
(a) Clinical Evidence.-A number of the patients who come under observation with gastric carcinoma give a history of gastric trouble extending over many years. The first step in the argument is the assumption, that the gastric symptoms are due to gastric ulcer. When, subsequently, on the operation-table, or in the post-mortem room, the existence of cancer is confirmed, the argument is carried a stage further by assuming, that the cancer has originated in the ulcer, which, from the history, is supposed to have existed previously.

The argument may be summarised briefly thus: Cancer is a disease, the duration of which is comparatively short; a long history of gastric trouble is evidence of the presence of an ulcer; therefore, when cancer is associated with a long history, the cancer must have been grafted on a simple ulcer. Such an argument is unscientific. It contains two fallacies, and disregards an alternative conclusion, at least of equal probability.

The first fallacy is, the assumption that we can diagnose a gastric ulcer from the clinical history alone. In duodenal ulcer, it is true, the history often is so typical, that from it a diagnosis may be made with confidence. This is not so with gastric ulcer. Usually the diagnosis of duodenal ulcer is easy, that of gastric ulcer very difficult. From operative experience we have learnt that gastric ulcer is far less common than formerly was supposed. The symptoms attributed to gastric ulcer frequently are due to disease of the appendix, or of the gall-bladder, to septic gastritis, or to intestinal toxæmia. Although in some instances, when dealing with a particularly intelligent patient, it may be possible from the history alone to differentiate between these conditions, in the majority of cases it is not so. I maintain, therefore, that because a patient with cancer of the stomach gives a long history of dyspeptic trouble, we are not justified in 
assuming that the symptoms have been due to a gastric ulcer. The second fallacy is the assumption that the duration of carcinoma is never protracted. It is conceivable that the growth of cancer in its early stages is much slower than we think. At present we have no means of determining the life-history of cancer of the stomach. We do know, however, that in some instances it is of slow growth, and that dissemination is a late manifestation. Most surgeons have met with instances, in which, after gastro-jejunostomy for inoperable cancer of the stomach, the patient has lived for years before succumbing to the disease.

Some years ago I performed gastro-jejunostomy on a woman, aged twenty-seven years, who had an extensive pyloric cancer with such widespread glandular infection, that a radical operation was impossible. A year later she had gained nearly three stone in weight, and was so well, that when the American Society of Clinical Surgery was in this country, I showed her to the members as a case in which I had been mistaken as to the nature of the disease. The patient remained well for two years, then exhibited signs of sudden, rapid growth of the disease, and died within a few months.

A more remarkable case is that of a man, aged fifty-two years, upon whom I performed gastro-jejunostomy in 1908. He had cancer of the pylorus, which had spread in the submucous tissue almost up to the cardiac end of the stomach. Radical operation was impracticable. I removed a portion of the growth, and microscopically it proved to be a spheroidal-celled carcinoma. It is now more than $4 \frac{1}{2}$ years since the operation, and the patient states that " he is as well as he has ever been in his life." Until we know more of the natural history of gastric cancer, we are not justified in drawing conclusions as to whether life is, or is not, greatly prolonged in some instances. 
The alternative to the conclusion that carcinoma is grafted on ulcer is, that simple ulcer and carcinoma may occur independently in the same patient. That sometimes this should happen is highly probable, for the frequency of the incidence of cancer and ulcer is very similar. Further, it is a hypothesis based on fact. On several occasions, when operating for cancer, I have been able to demonstrate to those present, either an open ulcer, or the scar of an ulcer, quite distinct from the malignant growth. In several, at least, of the cases recorded as instances of ulcus carcinomatosum, two ulcers were observed, one simple and the other malignant.

A perusal of the records of cases of ulcus carcinomatosum reveals one striking feature, namely, the frequency with which there is an interval between the symptoms of supposed ulcer and the onset of the symptoms of gastric cancer. With regard to such cases as these, the assumption that cancer has been grafted on an ulcer is less reasonable than the hypothesis, that an ulcer has been present and has become healed, and subsequently cancer has occurred independently of the ulcer.

(b) Pathological Evidence-According to Drs. Wilson and MacCarty, in 71 per cent. of their series of cases there was " a large ulcer with scar tissue centres and overhanging borders, deep in the bases of which cancer is present, which ulcer in almost every instance has unmistakably originated in the lesser curvature of the stomach, the usual site of gastric cancer. Further, almost every case gives a clinical history suggesting gastric ulcer for a long period of years preceding the relatively short period when the history became that of gastric carcinoma." Apart from the assumption that a previous history of gastric trouble is evidence of gastric ulcer-an assumption which I have criticised already - the weak point of this argument appears to me to be 
absence of proof that an ulcer in which cancer cells are present, has ever been other than malignant. The presence of scar tissue is not necessarily evidence of non-malignancy. We know that in cancer of the breast there may be formation of scar tissue, Nature's attempt at arrest of the disease. Further, the border line between a benign and a malignant growth is a narrow one, and a given tumour may be inherently malignant before, clinically or pathologically, we can recognise its malignant character, with the means at present at our disposal.

There are two truths which appear to me to make it very difficult to accept the view, that the grafting of cancer on ulcer is of frequent occurrence. First, simple ulcer of the duodenum is at least as common as gastric ulcer, and yet cancer of the duodenum is a rare disease. Surely, if simple ulcer be a frequent precursor of cancer, then cancer of the duodenum should be at least as frequent as gastric cancer. Then again, cancer is commoner in the second than in the first portion of the duodenum. Ulcer in the second portion is the rare exception. If ulcer be the precursor of cancer, then cancer should be far commoner in the first than in the second portion. Exactly the contrary is the case.

Secondly, death from cancer, after gastro-jejunostomy for supposed simple ulcer, is a rare event. If the hypothesis as to the frequency with which simple ulcers become malignant be correct, then many of the ulcers supposed to be simple should prove later to be malignant, and consequently, many of the patients on whom gastro-jejunostomy is performed, should die subsequently from cancer. As a matter of fact, the mistake, when made, is more often in the opposite direction; it is the supposed malignant growth which proves to be innocent, not the supposed simple ulcer which proves to be malignant. My own experience is that only one per cent. of the patients on whom gastro-jejunostomy is per- 
formed for simple ulcer, die later from carcinoma. Professor Kocher has published recently, a review of fifty cases of gastro-jejunostomy. The period which had elapsed since operation varied from two to twelve years. In no case was there a recurrence of symptoms, or any suggestion of malignancy. Dr. Gressot states that malignant degeneration of surgical ulcers occurs in only $2 \cdot 3$ per cent. of all cases after gastro-jejunostomy.

In view of such observations it is difficult to accept the view, that grafting of cancer on simple ulcer is a frequent event. Of course I do not deny that a simple ulcer may become malignant. My point is, as regards the frequency of such a happening. That it is anything like so common as some authorities teach, in my opinion is doubtful. The subject requires much further investigation. In the meantime the verdict should be, the Scotch verdict of "Not proven."

\section{REFERENCES}

Brinton, Dis. of Stomach, p. 192.

Cruveilhier, Path. Anat., liv.

Dieulafoy, Presse Médicale, 1897, p. 289.

Dittrich, Präger Vierteljahr, xvii. p. 6.

Fenwick, S., Cancer of the Stomach, 1902, p. 216.

Goodhart, Trans. Path. Soc., xl. p. 78.

Graham, C., Collected Papers of the Mayo Clinic, i. p. 112.

Gressot, Berlin. Klin. Woch., xlix., 1912, No. 22.

Koch, Med. Woch, Petersb., 1894.

Häberlin, Archiv f. Klin. Med., 1889, p. 46.

Hauser, Das Chronische Magengeschwür, 1883.

Hayem, Presse Médicale, 1898, ii.

Kelynack, Brit. Med. Jour., 1897, i. p. 18.

Lebert, Magen. Krankheiten, p. 339.

Maillefert, Diss. Greifswald, 1895.

Mayo, W. J., Collected Papers of the Mayo Clinic, i. p. 62.

Murrell, Med. Press and Circular, Oct. 23, 1901.

Riegel, Zeit. f. Klin. Med., 36.

Rosenheim, Zeit. f. Klin. Med., 1891.

Wilson and MacCarty, Amer. Jour. Med. Sciences, 1909, cxxxviii. p. 846. 


\section{CHAPTER XIX}

\section{INFANTILE HYPERTROPHIC PYLORIC STENOSIS}

I USE the term " infantile" in preference to " congenital " as the hypothesis, that the disease exists before birth, has not been verified. It is only within recent years that the disease has been recognised clinically. According to Sir William Osler, the earliest record of a case is that described by Hezekiah Bardsley in the year 1788, under the title of "A Case of Scirrhus in the Pylorus of an Infant." Additional cases were reported by Williams in 1841, and Daworsky in 1842 , but these isolated records were forgotten until the year 1888, when Hirschsprung drew attention to the subject anew.

It is likely that the disease is more common than recorded cases and museum specimens would lead us to believe. Probably many of the patients suffering from the disease are treated without the true cause of the symptoms being ascertained.

Morbid Anatomy.-The essential feature in all recorded cases and museum specimens is a marked thickening of the pylorus. This thickening is due to a hypertrophy, or rather hyperplasia of its circular muscular fibre, without any evidence of inflammation. The mucous membrane lining the pylorus is thrown into folds. Dr. Cautley and Mr. Dent, in a valuable paper, point out that a single reduplication of the mucous membrance, much more marked than any other fold, is a conspicuous feature in many of the specimens (Plate XXX, Fig. 70). Both these writers and Dr. John 


\section{Plate XXX.}

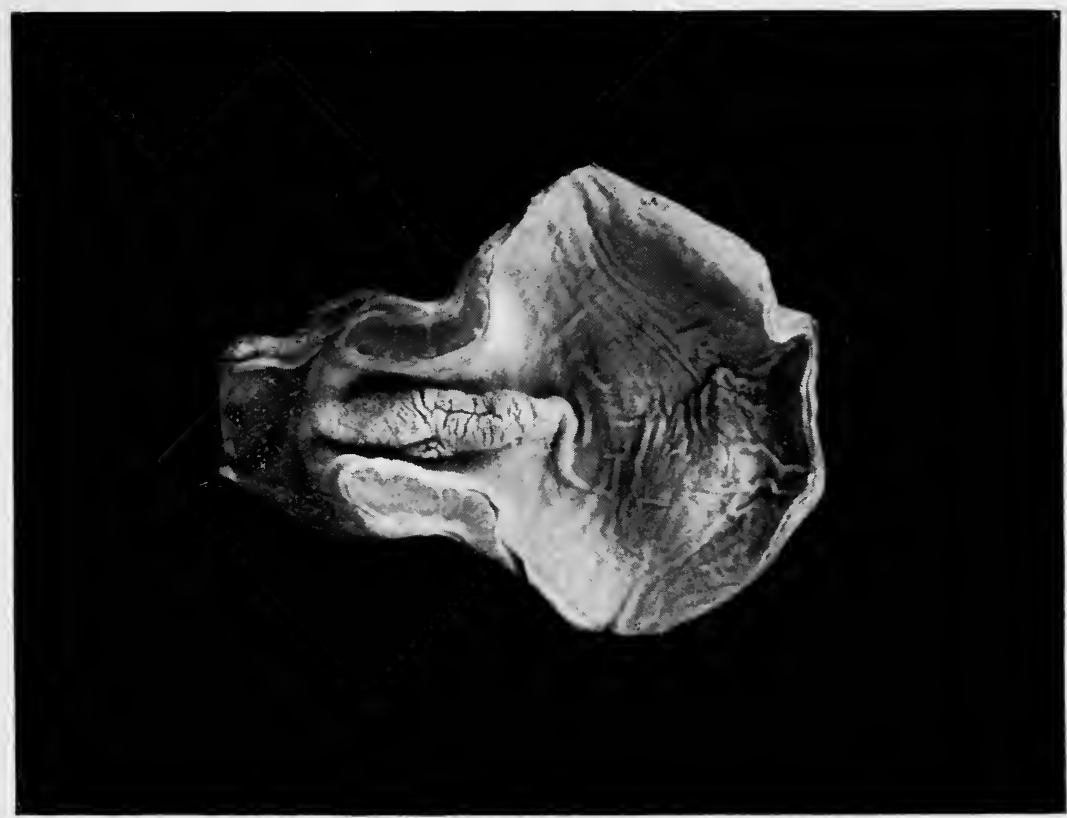

FIG. 70.-Infantile pyloric stenosis. The fold of mucous membrane is well seen. (Royal College of Surgeons' Museum. No. A2398a.) 
Thomson agree that as a rule there is no thickening of the submucous coat. The thickening is usually more marked at the duodenal end of the pylorus, is often of cartilaginous hardness, and in length measures about one inch. Stenosis is not complete, for a probe can be passed readily through the pylorus, but during life obstruction probably is complete owing to the folds of mucous membrane described above.

Two curious features of the disease are, its frequency in male infants, and the relatively large proportion of firstborn children who are affected. About 80 per cent. of all cases occur in males.

Ætiology.-The ætiology of the disease is uncertain. Two hypotheses have been suggested :

I. That the thickening of the pylorus is a definite anatomical condition due to hyperplasia or hypertrophy of the muscular tissue. Some authorities regard the hyperplasia as primary, while according to others the increase of muscular tissue is of the nature of a hypertrophy secondary to spasm of the pylorus.

2. That the symptoms are not the result of an anatomical lesion, but of a functional spasm of the pylorus.

These views may be represented graphically thus:

$$
\text { Infantile Stenosis }\left\{\begin{array} { l } 
{ \text { 1. Anatomical } } \\
{ \text { 2. Functional } }
\end{array} \left\{\begin{array}{l}
a . \text { Primary hyperplasia. } \\
b . \\
\text { Secondary hypertrophy. }
\end{array}\right.\right.
$$

At the present time the available evidence is in favour of the view that the condition is a primary local overgrowthin other words, a freak of Nature. Dr. Cautley, who adopts this view, says that "Nature in her anxiety to provide an efficient pyloric sphincter has over-exerted herself, and produced too large a quantity of muscular tissue." In support of this view, it has been urged that when a child, affected with infantile pyloric stenosis, dies within a few weeks of 
birth, the amount of hypertrophy found is greater than could have been produced since birth (Rolleston). If the pyloric hyperplasia existed at birth, it must have been either a primary developmental abnormality, or due to spasm set up by the swallowing of amniotic fluid in utero. That a fœtus swallows amniotic fluid is at present pure assumption.

As against the view that the hyperplasia is primary, it is urged that hypertrophic pyloric stenosis never has been observed in a dissected fœetus. On the other hand, the occurrence of the disease in three members of the same family, as recorded by Henschell, at least suggests the possibility of a congenital origin.

Symptoms.-While authorities differ as to the ætiology of the disease, there is striking unanimity as to the clinical symptoms.

Usually the infants affected are healthy-looking babies of normal weight. The prominent symptom is vomiting. Within a short period, varying from a day or so to three or four weeks, the infant begins to vomit after its feeds. The vomit consists of altered food with a little mucus. Bile is not present. Change of milk has little or no effect on the vomiting. Dr. Robert Hutchison has pointed out a curious feature of the vomiting, namely, its tendency to cease for some hours with any change of food, and to recur again as violently as ever. At first the quantity vomited is small, and some feeds may be kept down, and later apparently the whole may be brought up at once. Gradually the vomiting increases in frequency and amount, and is often forcible in character, while the infant's weight steadily decreases, and its face becomes pinched and wizened.

The condition is distinguished from the gastro-enteritis, so common in children, by the absence of diarrhœa ; as little or no food passes through the pylorus, there is little fæcal 
residue to be evacuated, and so obstinate constipation is a marked symptom.

In the early stages of the illness, little can be made out from an examination of the abdomen, but later the stomach becomes dilated; the intestine being empty, the lower part of the abdomen is collapsed and hollowed out, so that the contrast between the lower part of the abdomen and the upper part, distended by the dilated stomach, is very characteristic.

At a later stage, the outline of the stomach may be made out definitely, and waves of contraction can be seen passing across the upper abdomen from left to right. In some instances a definite pyloric tumour may be felt, especially if the examination be made under an anæsthetic-a precaution which should not be omitted in a doubtful case.

Treatment.-There is little doubt that some patients recover without surgical intervention. Dr. G. F. Still has had under his care several undoubted cases, in which recovery has ensued after daily lavage of the stomach. In all of his cases there was a dilated stomach and a definite pyloric lump. In a case recorded by Dr. F. E. Batten, the diagnosis was verified subsequently by post-mortem examination, as the child, after gaining $8 \frac{1}{2}$ pounds in weight in five months, succumbed three months later to an attack of gastro-enteritis and pneumonia. Dr. Hutchison records 18 recoveries in a series of 20 cases. Heubner has reported 21 cases treated medically with 19 recoveries, Starck 12 cases with 11 recoveries, Bloch six cases with recovery in all of them, and Bendix 32 cases with recovery in 30 . I myself have seen two patients recover with regular daily lavage.

These facts should make us pause before advocating operation. Further, it is important to note, that in the cases which have been treated successfully without operation, vomiting has diminished and finally ceased, but only after some weeks of regular treatment. 
Therefore a very thorough and extended trial should be given to careful feeding and regular lavage, before resorting to surgical measures. It must be admitted, that in hospital practice this treatment requires so much individual attention, that it is difficult to carry it out, while the impossibility, in an institution, of feeding the children by the breast, is a serious drawback; but in private practice, if the mother be unable to nurse the child, the services of a wet nurse can be requisitioned, in addition to the undivided attention of a skilled nurse.

It is noteworthy, that in many of the cases which have been treated successfully by Loreta's operation, vomiting has not ceased immediately, and the feeding of the infant has required much care and patience for some time. Had the patience been exercised before operation, possibly the necessity for operation might not have arisen. Present experience, then, seems to indicate that if these cases be recognised early, treated by systematic lavage, and fed by the breast, there is at least a possibility of recovery. Whether this recovery is absolutely permanent, time alone can show. At any rate, if operation can be delayed until the infant be older and stronger, something will have been gained.

Schwyzer, in 1897, was the first to propose, and Meltzer, in 1898, the first to practise, surgical intervention for this disease. Four different procedures have been adopted: namely, pylorectomy, gastro-jejunostomy, pyloroplasty, and pylorodiosis or Loreta's operation.

Pylorectomy may be dismissed at once as an unsuitable operation for such young patients. Mr. H. J. Stiles, who, acting on the opinion expressed by Meltzer, performed the only operation recorded, condemns it as unnecessarily severe and unjustifiable.

Gastro-jejunostomy.-The first successful gastro-jejunostomy for this disease was performed by Löbker in July 1898 . 
So far the mortality attending this operation in these cases has been very high, but it is only fair to point out that many of the little patients were very emaciated and in an extremely unfavourable condition for operation.

In most of the cases the posterior method was adopted, but my own opinion is, that in these very young patients, the anterior operation is attended with less risk. In my Hunterian lectures I recorded cases in which the patients were in excellent health six years after operation.

Pylorodiosis.-The mortality of pylorodiosis also is high. There is considerable risk of rupturing the duodenum; on the other hand, if the stretching be not sufficient, it is extremely likely that relapse will ensue.

Pyloroplasty.-The first pyloroplasty was performed by Braun. In some instances the operation has proved very satisfactory, but in others there has been trouble with the feeding-a serious matter when the condition of the child before operation is considered.

The Choice of Operation.- So much turns on the condition of the infant at the time of operation, that it is impossible to estimate the relative mortality of these different procedures, and probably it is on this factor and on the skill of the operator, rather than on the particular operation adopted, that the immediate result depends.

The one thing certain is, that surgical intervention in these cases is a serious matter. Whatever operative method be chosen, the mortality rate is high. Therefore, every effort should be made, in the direction indicated above, to obviate the necessity for operation. On the other hand, we must guard against delay, until the child be in such condition that recovery is almost hopeless, as has happened in many of the recorded cases. Pylorodiosis appears to be the least severe procedure, but the after-results hardly can be considered satisfactory. It is only fair, however, to state that some 
surgeons have obtained satisfactory after-results by this operation. Mr. Burghard tells me that he has had only three instances of recurrence of symptoms in the twenty-five patients who recovered after operation. In two of the cases, a second operation was necessary; in the third case, the patient recovered with lavage.

The mortality rate of all the procedures available is so high, that it is difficult to decide which is the best. My own view is that the choice lies between pyloroplasty and gastrojejunostomy.

Pyloroplasty has been advocated warmly, and supported ably, by Cautley and Dent. They maintain that it is a less severe operation than gastro-jejunostomy, and that it can be performed through a small incision, and that it brings about as complete a recovery as gastro-jejunostomy. To this I would say, that pyloroplasty is not always so easy as its advocates would have us believe. Even in an adult, it is difficult to be certain as to the size of the channel left after suturing, and this difficulty is much greater in an infant; it is significant, that in two of the recorded cases complete pyloric obstruction resulted from the operation, a complication which I think is likely to occur in a considerable proportion of the cases.

With our present knowledge it is difficult to decide whether pyloroplasty, or gastro-jejunostomy be the better operation. Whether the remote results will prove as satisfactory after pyloroplasty, as after gastro-jejunostomy, we do not know. I agree with Mr. Stiles, that in principle gastrojejunostomy is the better operation, and the following are my reasons :

I. It is preferable to operate on normal than on morbid tissues.

2. Feeding can be commenced at once after gastrojejunostomy, no small advantage in these cases, whereas in 
the cases of pyloroplasty the feeding has caused no little anxiety.

3. If the anterior operation be performed, it can be completed in twenty-five minutes, which is but little, if at all, longer than the time required for pyloroplasty.

4. The incision need be no longer than that required for pyloroplasty ; $2 \frac{1}{2}$ inches is quite sufficient. If the patient's shoulders be raised, there is no fear of protrusion of the intestine.

5. The remote results of gastro-jejunostomy in these cases are excellent. We do not know yet that those of pyloroplasty are as good.

\section{REFERENCES}

Batten, The Lancet, Dec. 2, 1899, p. 1511.

Bendix, Med. Klinik, 1909, xlviii.

Block, Jahrbuch f. Kinderheilk., 1907, 1xv. p. 317.

Brenner, Wiener Klin. Woch., 1892, v. p. 375.

Burghard, Trans. Clin. Soc., 1907, xl. p. 98.

Cautley and Dent, Trans. of the Royal Med.-Chir. Soc., lxxxvi. p. 481.

Dawosky, Archives Génér. de Méd., 1843, ii. p. 93.

Fisk, Annals of Surgery, July 1906, p. 1.

Gardner, The Lancet, Jan. 10, 1903, p. 100.

Henschel, Archiv f. Kinterheilkunde, 1891, xiii. s. 32-57.

Heubner, Therapie der Gegenwart, Oct. 1906.

Hirschsprung, Jahrbuch f. Kinderheilkunde, 1888, xxviii. s. 61 .

Hutchison, R., Brit. Med. Jour., 1910, ii. p. 1021.

Löbker, Verhand. der Deut. Gesselsch. f. Chir., 1900, xxix. p. 148.

Osler, Boston Med. and Surg. Jour., Mar. 12, 1903.

Pfaundler, Wien. Klin. Woch., 1897.

Shattock, S. G., Proc. Roy. Soc. Med., ii., Pathol. Section, p. 88.

Starck, Zentralblatt f. Kinderheilk., 1909, xiv., No. 5.

Stiles, H. J., Trans. Roy. Med. Chir. Soc., loc. cit.

Thomson, Scottish Med. and Surg. Jour., June 1897, and Brit. Med. Jour., 1902 , ii. p. 678.

Wernstadt, Jahrbuch d. Kinderheilk., 1907, lxv. p. 674.

Wilcox, The Lancet, Dec. 14, 1907.

Williams, London and Edinburgh Jour. of Med. Sc., 1841, p. 23. 


\section{CHAPTER XX}

\section{FIBROMATOSIS OF THE STOMACH}

The term "fibromatosis" of the stomach has been suggested by Professor Alexis Thomson, to denote the condition which has been described variously as cirrhosis, linitis plastica, and fibroid induration of the stomach.

Pathology.-Aschoff regards cirrhosis as a sequel of chronic catarrh, the fibrous thickening extending from the mucosa to the outer coats of the stomach. Nothnagel expresses the view that it originates in, and spreads inwards from the peritoneal coat. Bret and Paviot maintain that the lesion always is cancerous, basing their conclusions, not on the microscopical examination of the submucosa, but on the condition of the perigastric lymphatic glands, and on the presence of metastatic deposits. Andral, Cruveilhier, Brinton, Wilson Fox, and Wilks take the contrary viewnamely, that the lesion is not malignant. These authorities regard it as a primary cirrhosis of the submucosa, inflammatory in nature, and analogous with cirrhosis of the liver. As Professor Thomson points out, its frequent association with chronic ulcer suggests an infective or toxic origin. In rare instances fibromatosis is associated with tubercle or syphilis.

The Differentiation of Fibromatosis from cancer of the stomach is often very difficult. The white appearance of the peritoneal coat, and the presence of enlarged glands, are extremely suggestive of carcinoma.

A number of specimens figuring in museums as cancer, and which have been removed as such, are probably examples 



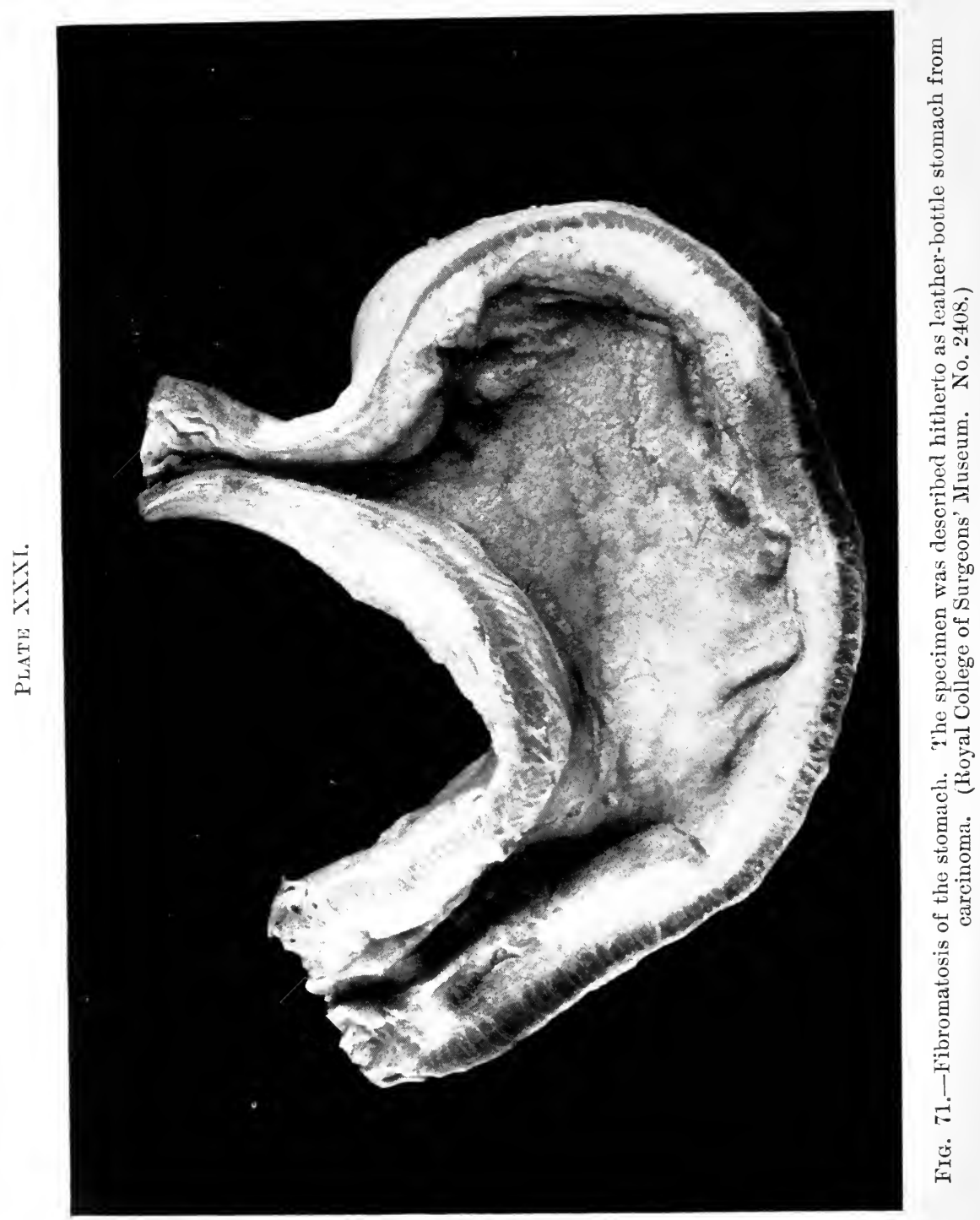


of fibromatosis. Professor Alexis Thomson recently has reexamined seventy-two specimens labelled as cancer, in the University of Edinburgh Museum, and found that in 26.3 per cent. of them the lesion was not cancerous.

Some years ago I came to the conclusion that the specimen of leather-bottle stomach (No. 2408) in the Museum of the Royal College of Surgeons, which has been figured so often as an example of leather-bottle stomach, the result of malignant disease, would prove to be an example of fibrosis (Plate XXXI, Fig. 71). Recently Mr. S. G. Shattock has been good enough to re-examine the specimen, and he reports that microscopical sections show no evidence of malignancy.

A stomach affected with fibromatosis is diminished in size, does not collapse when it is opened, and its capacity is much reduced. As a rule the thickening is most marked about the pyloric end of the stomach, and gradually tapers off towards the cardiac end.

There are three points of difference between fibromatosis and cancer, which usually can be made out plainly, if a section be made through the stomach wall.

First, in cancer there is no distinct differentiation of the layers of the gastric wall; in fibromatosis, the various layers are quite distinct.

Secondly, in cancer the infiltration is mainly in the mucous coat, in fibromatosis in the submucous coat.

Thirdly, in cancer, usually there is an ulcerated mass in the interior of the stomach, in fibromatosis no such growth is present.

As Professor Thomson has pointed out, in fibromatosis the stomach may become adherent to neighbouring organs, which may become infiltrated, thus increasing the resemblance of the disease to cancer. He describes such a case, in which the transverse colon was implicated. He resected 
the stomach and colon in the belief that they were the seat of cancer, and the patient made a good recovery.

Microscopical examination of the stomach walls shows that the submucous coat is replaced by fibrous tissue with newly-formed blood-vessels. The formation of fibrous tissue may extend both to the mucous layer and to the serous coats.

Treatment.-In the majority of cases in which surgical treatment has been adopted, resection has been performed in the belief that the disease was malignant. Probably a gastro-jejunostomy would give the patient relief, but I have been able to find the record of only one case in which this treatment has been adopted. Professor Roux of Lausanne performed anterior gastro-jejunostomy on a man aged thirty-two years. The patient was quite well $3 \frac{1}{2}$ years later.

The important point with regard to these cases is, that gastrectomy should not be performed until the diagnosis is certain. In cases of doubt a portion of tissue must be removed for microscopical examination.

Professor Thomson, in his excellent paper, recommends that the stomach be opened and its interior examined. In this way, in some of the cases at least, fibromatosis can be distinguished from cancer.

\section{REFERENCES}

Andral, Précis d'anat. Path., i. p. 47.

Bret and Paviot, Rev. de Méd. Paris, May 10, 1894, p. 384.

Brinton, Diseases of the Stomach, 1864.

Cruveilhier, Anat. Path., iii. p. 599.

Fox, Wilson, Diseases of the Stomach, 1872.

Hutersham, Diseases of the Stomach, 1869.

Hoche, Rev. de Méd., 1903, xxiii. p. 944.

Hoche and Vautsin, La Presse Médicale, 1903, ii. 477.

McCrae, Bull. Johns Hopkins Hosp., 1901, xii. p. 25.

Roux, Rev. Méd. de la Suisse Rom., 1905, xxv. p. 53.

Sheldon, Annals of Surgery, 1904, xxxix. p. 341.

Thomson, Alexis, Brit. Med. Jour., 1910, ii. p. 949.

Wilks, Trans. Path. Soc., 1862, xiii. p. 83. 


\section{CHAPTER XXI}

\section{BENIGN TUMOURS OF THE STOMACH}

BENIGN tumours of the stomach are exceedingly rare.

The following varieties have been described :

\section{Adenomata. \\ 2. Myomata. \\ 3. Fibromata. \\ 4. Lipomata. \\ 5. Lymphadenomata.}

Benign tumours of the stomach may :

(a) Grow towards the interior of the stomach, and project into its cavity (intra-gastric tumours, polypi).

(b) Remain encapsuled in the wall of the stomach.

(c) Grow towards, and project on, the external surface of the stomach, to which they may be connected by a pedicle (exo-gastric tumours).

1. Adenomata are seen as rounded or lobulated projections from the interior of the stomach. They may be single or multiple, and are commonly found near the pylorus, attached to the gastric wall either by a broad base or by a narrow pedicle. They undergo cystic degeneration readily, and often are described as mucous polypi. They consist of a central core of connective tissue containing blood-vessels and lymphatics, over which is spread a thin layer of unstriped muscular tissue. They are covered with greatly hypertrophied mucous membrane.

Cases are on record in which adenomata have caused 
pyloric obstruction. Some of the cases recorded as adenomata were probably adeno-carcinomata.

2. Myomata of the stomach, first described by Morgagni, occur as smooth, hard, round swellings in the gastric wall. They may grow either into the cavity of, or towards the outer surface of the stomach, and may become pedunculated. Occasionally they attain to a large size, and are recognised as abdominal tumours during life. Von Erlach removed successfully a tumour weighing thirteen pounds from the front wall of a woman's stomach, to which it was attached by a pedicle. Professor Von Eiselsberg removed a large fibromyoma, the size of a man's head, which was attached to the greater curvature. The patient recovered.

Deaver and Ashurst have collected forty cases of gastric myoma. About half of them were growing from the external surface of the stomach. In fourteen recorded cases of gastric myoma, secondary deposits were found in four instances-" leio-myoma malignum."

3. True fibro-myomata are exceedingly rare; tumours so described usually have proved to be fibro-sarcomata. Dr. Soltau Fenwick states that there is " not a single case in the whole of the literature where a large fibroid tumour of the gastric wall was above the suspicion of malignancy." Mr. Walter Spencer, however, has described a pure fibroma which he removed with success.

4. Lipomata occur in the form of the lobulated tumours characteristic of lipomata elsewhere. They originate, either in the submucous layer and project into the cavity of the stomach, or in the subserous layer and grow from the external surface of the stomach. In either situation they may become pedunculated.

5. Lymphadenoma of the stomach is a very rare condition. The mucous membrane of the stomach is thrown into polypoid folds, affecting chiefly the middle and cardiac ends 
Plate XXXII.

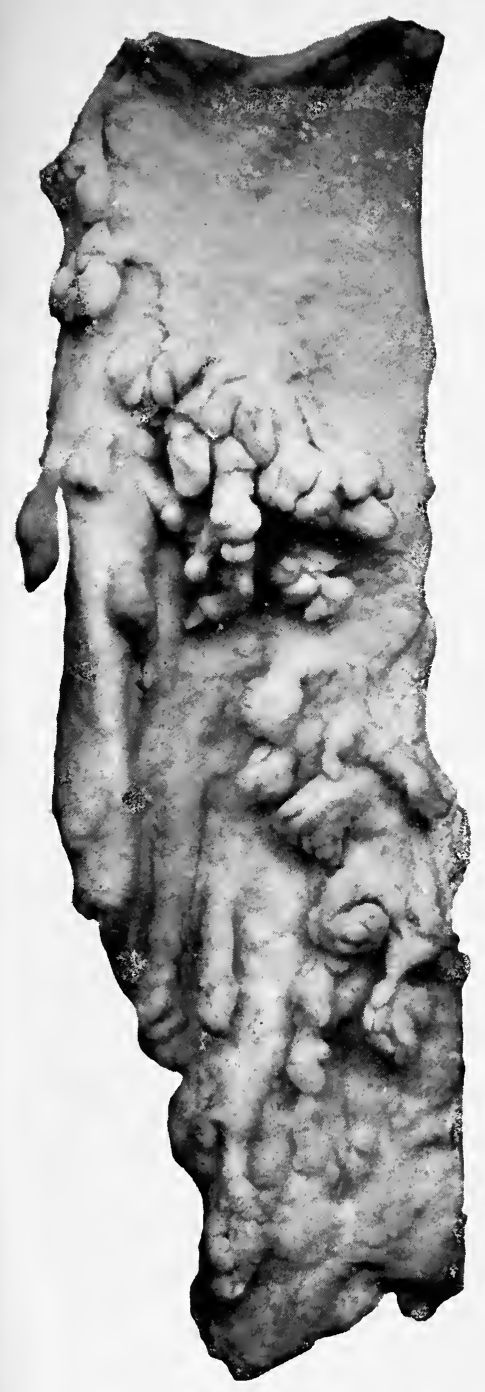

FIG. 72.-Mucous polypi in the stomach. (Soltau Fenwick.)

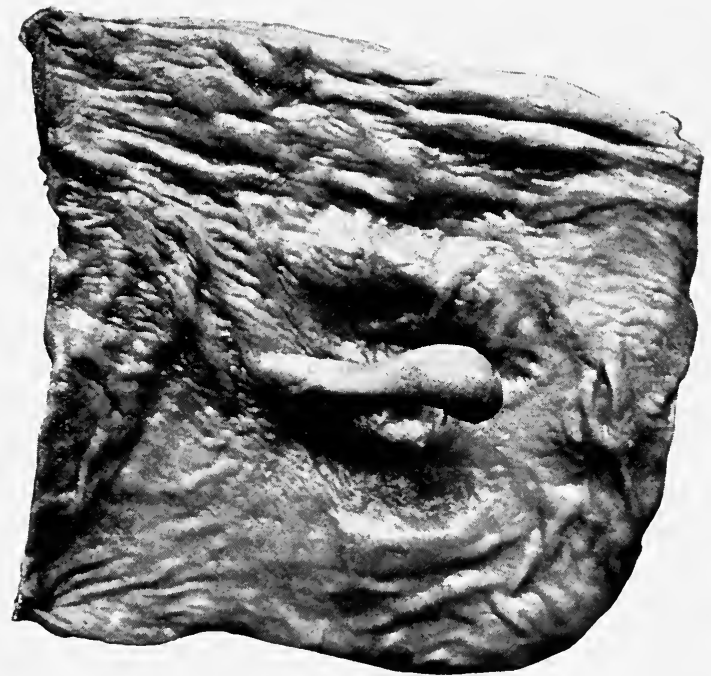

Fig. 73.-Pedunculated fibroma attached to the edge of the pyloric orifice. (Soltau Fenwick.) 
BENIGN TUMOURS OF THE STOMACH 267

of the stomach. The growths consist of lymphoid tissue. The mucosa overlying the growth may become infiltrated and ulcerated.

Lymphadenoma of the stomach is rare before middle life, but a case occurring in an infant eighteen months old has been recorded by Drs. Rolleston and Latham.

Symptoms and Treatment.-As a rule, benign tumours of the stomach give rise to no symptoms, and seldom are recognised during life. The intra-gastric tumours, if situated near the pylorus, may cause obstruction, and lead to such emaciation that the patient is supposed to be suffering from malignant disease.

Occasionally, benign tumours attain to such a size that they are palpable on examination of the abdomen: the chief, often the only symptom, in such cases, is abdominal discomfort. Owing to their mobility, the exo-gastric tumours, when of large size and attached to the stomach by a pedicle, may give rise to difficulty in diagnosis.

On several occasions gastric myomata in women have been diagnosed as ovarian adenomata (Maylard, Herman, Cantwell, Eiselsberg).

When single, benign tumours are amenable readily to surgical treatment. The important point is, that their benign character may not be recognised, and that partial gastrectomy may be performed, when it would be sufficient to shell out the tumour. Thus Von Hacker performed partial gastrectomy, and Hahn pylorectomy, in the belief that they had to deal with sarcomata, whereas subsequent examination demonstrated that the tumours were benign.

It is said that these tumours, if untreated, ultimately become malignant. It is more probable, that from the first, such tumours are sarcomata of slow growth. 


\section{Adenomata}

Andral, Grundriss der Path. Anat., 1830, ii. p. 33.

Beardsley, Trans. Path. Soc., viii. p. 219.

Brisaud, Arch. Gén. de Méd., 1885, p. 257.

Chaput, Bull. Soc. Anat., 1895, p. 534.

Collier, Trans. Path. Soc., xlvii. p. 46.

Cruveilhier, Anat. Path., xxii., pl. lvi.

Ebstein, Arch. Anat. u. Physiol., 1864, p. 94.

Leudet, Bull. Soc. Anat., 1850, p. 179.

Mérétner, Arch. de Physiol., 1888, ii. p. 32.

Norman, Jour. Med. Science, Dublin, 1893, p. 346.

Richard, Bull. Soc. Anat., 1846, p. 209.

Robson and Moynihan, Dis. of Stomach., 1904, p. 76.

\section{Myomata}

Cutler, Boston Med. and Surg. Jour., 1879, p. 513.

Eiselsberg, Arch. f. Klin. Chir., Bd. liv. p. 568.

Erlach, Wien. Klin. Woch., 1895, viii. p. 272.

Förster, Wien. Med. Woch., 1858, ix.

Goebel, Deutsch. Med. Woch., 1907, xxxiii. p. 1708.

Hacker, Wien. Klin. Woch., 1900, xiii. p. 145.

Hartmann, Bull. et. Mém. Soc. de Chir., 1905, xxxi. p. 817.

Herhold, Deutsch. Med. Woch., 1898, xxiv. p. 61.

Herman, Trans. Obstet. Soc., xliii. p. 2.

Kidd, Trans. Path. Soc., 1884, p. 196.

Kuntze, Arch. f. Klin. Chir., 1890, xl. p. 753.

Morgagni, De sedibus et Causis Morborum, Ep. xix., c. 58.

Poirier, Rev. de Chir., 1902, xxv. p. 485.

Sainter, Deutsch. Med. Woch., 1904, xxx. p. 914.

Steiner, Beit. zur Klin. Chir., 1898, xxii. p. 1.

Wesener, Virch. Arch., xciii. p. 377.

Yates, Annals of Surgery, 1906, xliv., p. 599.

\section{Fibromata}

Cleghorn, New Zealand Med. .Jour., 1892, p. 55.

Fenwick, S., Cancer of the Stomach, 1902, p. 350.

Spencer, Trans. Surg. Sect. Royal Soc. Med., ii. No. 9, p. 311.

Ware, Boston Med. and Surg. Jour., 1858, p. 83.

\section{LiPOMATA}

Fischer, Annals of Surgery, 1905, xliii. p. 583.

Murray, Trans. Path. Soc., 1889, xl. p. 78.

Russdorf, Deutsch. Klinik., 1867, p. 115. 


\section{BENIGN TUMOURS OF THE STOMACH 269}

\section{LYMPHADENOMA}

Arnott, Trans. Path. Soc., xxv. p. '150.

Coupland, Trans. Path. Soc., xxviii. p. 127.

Kredel, Berlin. Klin. Woch., 1883, p. 769.

Pitt, Trans. Path. Soc., 1889, xl. p. 80.

Rolleston and Latham, Lancet, 1898, i. p. 1313.

Silcock, Trans. Path. Soc., xxxv. p. 348.

Taylor, ibid., xxviii. p. 135. 


\section{CHAPTER XXII}

\section{APPENDICULAR GASTRALGIA}

That there are cases in which disease of the appendix mimics gastric disease, in some cases with very remarkable fidelity, does not admit of doubt. This does not appear to have obtained general recognition in this country, although abroad it has been recognised for some years. Twelve years ago Ewald referred to such cases under the name "appendicitis larvata," and Senator used the term "atypische appendicitis " for them. In America the rôle of the appendix in the production of gastric disorder has been recognised, not only in the work of the Drs. Mayo, but in the writings of Drs. Murphy, Ochsner, Deaver, and others. Two years ago I read a paper on this subject before the Royal Society of Medicine, and suggested that the term "appendicular gastralgia" is a convenient and descriptive one to apply to this condition.

\section{Symptoms}

Pain, varying in degree, is the prominent symptom in these cases. Some of the patients complain of what may be described as severe, continuous discomfort rather than pain, while some describe their pain as severe. In a large majority of the patients, the pain follows the ingestion of food, although the interval between the taking of a meal and the onset of pain is very variable. Some patients state that the pain comes on immediately after taking food, while in about one-third of the cases the interval between taking food and the onset of pain, varies from half an hour to three 
hours. In most cases the pain is referred to the epigastrium, usually to the right of the middle line. Some of my patients stated that the pain radiated downwards to the right iliac fossa, while, with less definiteness, others said that the pain radiated downwards. In one interesting case the pain radiated to the left iliac region, and at the operation the cæcum and appendix were found in the left iliac fossa. This radiation of the epigastric pain to the lower part of the abdomen is a most important diagnostic symptom, and in my experience, is one which never occurs in gastric, or duodenal ulcer. Occasionally very definite " hunger pain" is a symptom. Sour eructations and vomiting are present in considerably more than half of the cases. Flatulence and a feeling of distension after food is a very constant feature. Hæmorrhage occurs as an occasional symptom (15 per cent. of my cases), and I think there can be little doubt that the hæmorrhage comes from the gastric mucous membrane. In one case, in which I opened the stomach, the whole of the mucosa was studded with numberless bleeding-points. The interior of the stomach was in the condition described as " weeping blood." Probably this condition of the mucosa is due to the irritation of hyper-acid gastric juice, or to the continual presence of gastric juice in the fasting stomach (hyper-secretion).

Periodical Exacerbations.-A noteworthy feature of these cases is, that in many instances (67 per cent.); there is a periodical exacerbation of the symptoms. As a rule the patients are not quite well during the interval between the attacks, but suffer from discomfort and flatulence after food.

Another striking feature is the long history in a large number of the cases. The average duration of illness in my cases is six years, although in one or two of them there was an interval of one or two years between the attacks. Tender- 
ness in the epigastrium, usually to the right of the middle line, is an almost constant feature. In one-third of the cases there was tenderness over the appendix area as well as in the epigastrium. By far the larger majority of the patients are women.

Gastric Analysis. - The gastric findings are fairly constant. As a rule there is diminution or absence of free hydrochloric acid, but very occasionally there is hyperchlorhydria. The total acidity is usually above normal, the total chlorides are diminished, and the volatile acids slightly increased. Usually there is an excess of mucus, and sometimes the gastric contents are very thick and viscid. Hypersecretion is present in a considerable proportion of the cases.

The following is an illustrative case :

Female, aged thirty-seven years. Ten years ago the patient began to suffer from pain in the abdomen. She was said to have a "gastric ulcer." Ever since, she has suffered from pain coming on after food, with occasional free intervals. She used sometimes to make herself sick to relieve the pain. For the last three months she has been worse, as she always suffers from pain coming on an hour or less after food. She has had no vomiting, but has had sour eructations, and much flatulence. She is very constipated. In the morning the stomach contained 30 c.c. of yellowish fluid, but no food residue. After test meal:

\begin{tabular}{|c|c|c|c|c|c|}
\hline Total acidity & • & • & • & . & 75 \\
\hline Total chlorides & & • & 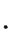 & . & $0 \cdot 318$ \\
\hline Free $\mathrm{HCl}$ & • & $\cdot$ & • & 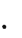 & 0.010 \\
\hline Protein $\mathrm{HCl}$ & • & $\cdot$ & • & • & $0 \cdot 210$ \\
\hline Mineral chlorides & & • & • & 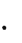 & $0 \cdot 098$ \\
\hline Volatile acids & . & - & . & . & $6 \cdot 8$ \\
\hline
\end{tabular}

At the operation the appendix was found to be bulbous, and bound down by dense adhesions. It contained a concretion. The mucosa was chronically inflamed, and in places hæmorrhagic. After operation her symptoms disappeared, and there was no hyper-secretion, the stomach being empty in the morning. 
The Operation Findings. - As regards the condition of the appendix, as a rule, judged by its external appearance, it is not extensively diseased. The most common condition is that the appendix is bulbous, somewhat thicker than usual, and, on opening it, a narrowing or constriction is found near the base, the distal side of the constriction being slightly dilated and giving rise to the bulbous appearance. A concretion was found in exactly half my cases. In all of my cases, microscopic examination of the appendix showed the existence of chronic inflammation.

In some of the cases the disease of the appendix is more extensive than that described above. There may be minute hæmorrhages in the mucous membrane, or a definite ulcerated area of the mucosa. In one of my cases the appendix was enormously dilated, measuring $3 \frac{1}{2}$ inches in circumference, the mucous membrane was ulcerated, and there were three large, very foul-smelling concretions in the lumen, as well as some blood-clot. This patient had had hæmatemesis as well as melæna.

There can be no doubt that an appendix, which on cursory examination, appears to present no abnormal features, may be the seat of disease fraught with grave danger to its possessor. On a number of occasions I have removed an appendix which, by an onlooker, might be regarded as " normal," and yet, subsequently, have been able to demonstrate to those present, the existence of disease within. Such an appendix usually presents the following features: It may not be obviously larger than usual, but inspection shows, that instead of being a uniform pale yellowish colour, its surface is covered with a branch-work of minute blood-vessels-evidence of chronic inflammation. Careful palpation may detect that the appendicular coats are thicker than usual. On cutting open the appendix, usually it is found to contain fæces, or a concretion, the mucosa is con- 
gested, sometimes petechial, or even definitely ulcerated. Microscopic examination shows the existence of chronic inflammation. However innocent-looking externally, an appendix which contains a concretion, or the mucosa of which is so damaged as to permit of bacterial invasion, is one which, at any time, may lead to a sudden attack of appendicitis.

The evidence that the gastric symptoms are due to the appendicular disease is threefold. First, the majority of the patients are cured by appendicectomy. The pain and discomfort are relieved, and the vomiting ceases. Secondly, usually there is an improvement in the character and quality of the gastric contents, which indicates the close physiological connection between the stomach and the appendix. Thirdly, the frequency with which patients who come under observation with an acute attack of appendicitis, give, on cross-examination, a history of previous gastric trouble, is certainly significant. A number of these patients state, that for years they have suffered from what they describe as bilious attacks-that is, attacks of vomiting, with discomfort after food, in the intervals between the attacks. More frequently, the patients state that for a considerable time before the acute attack of appendicitis, they have had to be careful as to their diet, otherwise they have suffered from indigestion. Dr. Soltau Fenwick has pointed out that before he recognised the connection between appendicular disease and gastric symptoms, he was acquainted with a peculiar type of hyper-secretion in which, frequently death occurred from appendicitis. Some years ago I had an interesting case of this type. I operated on a lady who was supposed to have a gastric, or duodenal ulcer. On opening the abdomen I could find no lesion of the stomach or duodenum. The symptoms were clear and definite, and, as the patient had had hæmorrhage, I thought that an ulcer must exist undiscover- 
able from external examination of the stomach. Accordingly I opened the stomach-a procedure of which, by the way, I now most strongly disapprove-and explored it from within. I found the whole mucous membrane in a state of extreme congestion, and studded all over with hundreds of minute bleeding-points. Accordingly I performed a gastrojejunostomy, and the patient was relieved at once of her symptoms, remained perfectly well for about eighteen months, but then had an attack of acute appendicitis, and narrowly escaped with her life. At that time I did not recognise the association of appendicular disease with the causation of gastric symptoms, but on looking back I have little doubt that the original gastric symptoms were secondary to chronic disease of the appendix. In this particular case gastro-jejunostomy was a success in so far as it relieved the patient of her gastric symptoms; nevertheless, I am convinced that I made a mistake, in that I ought not to have performed gastro-jejunostomy, but to have removed the appendix. Some years ago I saw two cases of gastric catarrh in children, due to latent disease of the appendix, which I did not recognise. In one of these the gastric catarrh was attributed to adenoids, and the boy was sent to me by a well-known physician to have his adenoids removed. This was done, but the boy was not a great deal better, and five months later he had a very acute attack of appendicitis. After appendicectomy he rapidly improved, and since has remained well. The other case was that of a girl who, for several years had been subject to attacks of gastritis without obvious cause. Later, she had definite tenderness over the appendix, and appendicectomy was performed. Since the operation, eight years ago, she has had no further trouble.

Differential Diagnosis.-The chief conditions from which appendicular gastralgia has to be distinguished are duodenal ulcer, gastric ulcer, and gall-stones. Of these, duodenal 
ulcer is the disease which gives rise to the greatest difficulty in the differential diagnosis. The symptoms produced by duodenal ulcer, and by the cases of latent appendicitis to which I am referring, are very similar in many respects. There is often the same history of recurrent attacks; both duodenal ulcer and appendicular disease may give rise to hyperchlorhydria, and also to entire absence of free $\mathrm{HCl}$. Even "hunger pain" may be a symptom of appendicular disease. The points which aid us in distinguishing the two diseases appear to me to be: First, in duodenal ulcer the patient usually is free from symptoms between the attacks ; in appendicular gastralgia, even between the attacks, the patient suffers from flatulence and discomfort after food. Secondly, the radiation of the epigastric pain towards the lower part of the abdomen is highly suggestive, if not diagnostic, of appendicular trouble. Thirdly, the existence of tenderness over the appendix sometimes throws light on an otherwise doubtful case. Fourthly, in many cases of appendicular gastralgia the alteration of the gastric contents is not commensurate with the severity and duration of the symptoms. Let me explain what I mean by this. In a number of the cases which I have examined, the gastric contents have been little altered chemically. This is not often the case in duodenal ulcer. In my experience duodenal ulcer most commonly produces, or is associated with, hyperchlorhydria, at any rate in the early stages ; later, occasionally, there is absence of free HCl. Appendicular disease apparently may exist for a considerable time without a marked alteration of the amount of free $\mathrm{HCl}$. The combination of marked symptoms of duodenal or gastric ulcer, with a negative gastric analysis, is suggestive of chronic appendicular disease. The cases of appendicular gastralgia associated with hyperchlorhydria present the greatest difficulty in diagnosis. My impression is that hyperchlorhydria 
secondary to appendicular disease does not give rise to the severe pain which usually is associated with the hyperchlorhydria which accompanies duodenal ulcer. Fifthly, a point on which too much stress should not be laid, is, that duodenal ulcer is far more common in men; appendicular gastralgia appears to be more common in women.

In gastric ulcer, food, especially milk, often gives relief for the time being, the pain recurring one or two hours later. In appendicular gastralgia the onset of pain is more variable; food, even milk, usually produces pain or discomfort at once. In gastric ulcer, usually the motility of the stomach is not impaired, unless the ulcer be near the pylorus, in which case often there is food retention. In gastric and duodenal ulcer the pain is rarely so severe and continuous that the patient has to take to bed. In several of my cases of appendicular gastralgia, the patients have had to lie up on account of the continuous and exhausting character of the pain. Another point which is helpful in the diagnosis is this: the administration of bismuth and alkalis, in my experience, has little or no influence on the pain and discomfort due to appendicular disease.

(For the points of difference between appendicular gastralgia and gall-stones, see p. 139.)

How are the Gastric Symptoms Produced?-When we attempt to explain why appendicular disease should produce gastric symptoms, we are entering into the region of speculation. I think I have brought forward evidence to show that there is a close connection between the appendix and the stomach, but as to the exact nature of this connection I am not prepared to dogmatise. Dr. W. J. Mayo thinks that appendicular disease causes a protective spasm of the pylorus. Possibly this is the explanation of those cases in which there is hyperchlorhydria. As Dr. Fenwick has pointed out, the symptoms are those of hyper-secretion. Exactly how the 
hyper-secretion is caused, we are not in a position to say. All we know at present is, that in some way, the appendix does affect the gastric secretion. Personally, I think that intestinal stasis, rather than pyloric spasm, is the condition in these cases. The symptoms may be regarded as toxic, the result of the intestinal stasis. This view seems to be supported by the frequency with which the duodenum is found markedly dilated at operation, and by the fact that in a number of the cases the stomach also is dilated. Further, as a rule, the quantity of gastric contents evacuated after a test-meal is greater than in healthy individuals, the percentage of volatile acids is increased, and, in addition, flatulence and constipation are prominent symptoms.

The Indications for Operation.-That latent appendicular disease gives rise to gastric symptoms is now established definitely. The symptoms are those of hyper-secretion or the so-called " acid dyspepsia." I need hardly state that I do not suggest that all, or even a majority, of the cases of acid dyspepsia require operation, unless the symptoms are persistent or recurrent. Persistent acid dyspepsia, in my opinion, is secondary to some definite organic lesion. When we have reason to believe that gastric symptoms are due to appendicular disease, then usually operation is advisable, provided that medical treatment has had a thorough trial and has failed. I hold it as a cardinal principle, that in all cases of gastric disorder, except when there is evidence of some gross lesion, such as carcinoma, pyloric stenosis, or hour-glass stomach, it must be premised that medical treatment has been tried and failed. And when I speak of medical treatment I mean, not a few weeks of drug-taking, but a course of rest and careful dieting, extended over at least six months.

It is the cases in which there are no physical or chemical signs, which require careful consideration. In those cases in 
which, in addition to the gastric symptoms, there is definite and persistent tenderness over the appendix, operation usually is advisable. I confess that I have an old-fashioned prejudice against operating when pain is the only symptom. At the same time, I recognise that there are such cases in which operation is not only justifiable but necessary. I take it that all are agreed that it is right to operate in cases of so-called "appendicular colic" when such attacks are recurrent. Surely it is equally justifiable to remove the appendix, when the patient suffers from recurrent attacks of gastralgia attributable to disease of the appendix, provided medical treatment has been tried and has failed. And in this connection I would point out again the diagnostic significance of radiation of epigastric pain to the lower part of the abdomen. I would insist, however, that the pain must be definite, and such that it interferes seriously with the patient's enjoyment of life.

\section{CONCLUSIONS}

(1) Appendicular disease may give rise to symptoms which mimic closely the supposed symptoms of gastric and duodenal ulcer.

(2) The prominent symptom is epigastric pain or severe discomfort after food ; in many cases there are sour eructations, vomiting, and even hæmatemesis and melæna.

(3) The radiation of epigastric pain to the lower abdomen is very suggestive of appendicular trouble.

(4) Gastric analysis reveals, in a few cases hyperchlorhydria, in others a normal amount of free $\mathrm{HCl}$, but in the majority a marked diminution or absence of free $\mathrm{HCl}$. As a rule there is an increase of the volatile acids, and, in many cases, evidence of hyper-secretion.

(5) Some cases of hyper-secretion or acid dyspepsia, and 
many cases of supposed gastric or duodenal ulcer, are cases of latent appendicular disease.

(6) The evidence that the gastric symptoms are due to appendicular disease is threefold: $(a)$ the majority of the patients are cured by appendicectomy; $(b)$ the influence which appendicectomy has on the gastric contents; $(c)$ the frequency of a history of previous gastric symptoms in those who have an attack of acute appendicitis.

(7) Probably the symptoms are the result of intestinal toxæmia due to intestinal stasis. In the early stages, the effect on gastric secretion possibly is due to pyloric spasm, but more probably to the controlling influence of the appendix on the effluent from the ileum.

(8) Appendicular gastralgia is apparently more common in women than in men.

(9) The important lessons to be learnt from these cases are: (i) That no operation should be performed on the stomach, except when a definite organic lesion of the stomach or duodenum exists. Gastro-jejunostomy will not cure appendicitis. (ii) That in all operations for supposed gastric or duodenal ulcer, the condition of the appendix should be investigated carefully.

\section{REFERENCES}

Ewald, C. A., Berl. Klin. Woch., 1889, xxiv. p. 532.

Fenwick, Soltau, Proc. Roy. Soc. Med. Surg. Sect., April 1910, p. 177.

Graham and Guthrie, Jour. Amer. Med. Assoc., March 1910, liv. p. 96.

Moynihan, B. G. A., Brit. Med. Jour., 1910, i. p. 241.

Paterson, H. J., Appendicular Gastralgia, 1910. 


\section{CHAPTER XXIII}

\section{SYPHILIS AND TUBERCLE OF THE STOMACH}

Syphilis of the stomach is rare, although probably more common than is generally believed. It is more frequent in men than in women, and the patients usually are between the ages of thirty and forty-five years. It is manifested in three forms-gummata, chronic gastritis, and pyloric stenosis.

1. Gummata.-Gummata originate in the submucous tissue, and rarely attain a large size. As a gumma increases in size it undergoes caseation, the overlying mucosa becomes infiltrated, thinned, and finally destroyed, resulting in a gummatous ulcer. Such an ulcer is irregular in shape, with thickened, undermined edges, its base covered with a yellow slough. The surrounding mucous membrane is of a reddish slate-colour, owing to chronic inflammation. Ulcers may also result from interference with the vascular supply of circumscribed areas of the gastric mucosa, due to obliterative arteritis.

2. Chronic Gastritis.-As Dr. Soltau Fenwick points out, chronic gastritis may ensue either as a direct, or as an indirect result of syphilis. The indirect variety is the more common, and " is due either to embarrassment of the gastric circulation from disease of the liver or spleen, to lardaceous degeneration of the vessels of the stomach, to secondary disease of the kidneys, or to the specific cachexia. Chronic inflammation of the stomach directly dependent on the systemic infection, occasionally results from repeated attacks of an acute character during the early phase of the com- 
plaint, but as a rule it appears only at an advanced stage of the disease, and is often associated with gummatous lesions of the bones, liver, or testes."

3. Pyloric Stenosis.-Pyloric stenosis may arise either from cicatrisation of ulcers in the neighbourhood of the pylorus, or from gummatous infiltration of the pylorus. It is a rare condition. I have seen only one example.

The patient, a man aged forty-three, was under the care of my colleague, Dr. Soltau Fenwick, suffering from pain coming on two hours after food, and vomiting. The vomiting gradually increased in frequency, until it occurred regularly once in the day and once during the night. The quantity vomited was often as much as two pints. He had a large, splashy stomach with visible peristalsis. On passing a tube in the early morning, four pints of fluid, containing much food remains, was recovered. Gastric analysis of a test-meal gave the following results :

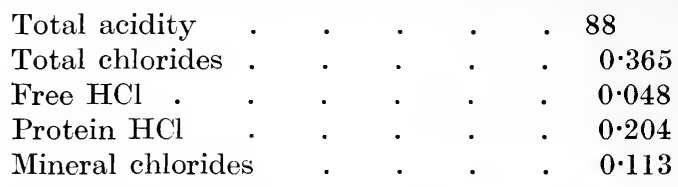

Dr. Fenwick diagnosed syphilitic disease of the stomach, and after a trial of anti-syphilitic remedies, advised operation. At the operation I found well-marked stenosis of the pylorus due to fibrous thickening. There was much fibrous thickening all round the liver, evidently the result of perihepatitis. I performed gastro-jejunostomy, and some years later, I heard that the patient was in perfect health.

Pyloric stenosis, the result of syphilitic disease in the vicinity of the pylorus, is a more common condition. I have met with two examples. In one case, that of a man aged thirty-three years, a gumma of the liver had become adherent to the first part of the duodenum, and caused obstruction. I drained the gumma and performed gastrojejunostomy, which was followed by complete relief. 
Symptoms.-The symptoms of a gastric ulcer due to syphilis are, pain after food and vomiting. Hæmatemesis is not common. As in all cases of visceral syphilis, anæmia is a prominent symptom, and often is very profound. The cachectic appearance of the patient is very striking. According to Dr. Soltau Fenwick these cases differ from the simple variety of ulcer in three particulars: the extreme severity of the pain and vomiting, the infrequency of hæmatemesis, and their resistance to ordinary treatment, and great tendency to relapse.

Treatment.-In cases of intractable gastritis, or gastric ulcer, the possibility of a syphilitic origin must be borne in mind, and if there be any suspicion of the presence of this disease, a Wasserman's test should be performed. If this be positive, a trial should be given to the administration of potassium iodide, and the intra-muscular injection of mercury, together with rest in bed and milk diet. If these prove ineffectual, or the symptoms recur, recourse should be had to gastro-jejunostomy. As has been pointed out by Dr. Fenwick, the possibility should be kept in view that the symptoms of gastric irritation in a syphilitic subject may be due, not to the specific malady, but to the prolonged administration of mercury, and potassium iodide.

In pyloric stenosis, medical treatment is of no avail; gastro-jejunostomy is indicated.

\section{Tubercle of the Stomach}

Primary Tubercle of the stomach is very rare. Usually it is secondary to, or associated with, tubercle of other organs.

The symptoms are indefinite, but may suggest carcinoma, to which the gastric findings bear a close resemblance.

As a rule, surgical treatment is of no avail, although two 
cases have been recorded of successful resection of the pylorus for stenosis of tuberculous origin.

\section{REFERENCES}

Batsère, Thèse de Toulouse, 1901.

Bittner, Centralblatt f. Allgem. Path., 1894, p. 175.

Blumer, Brit. Med. Jour., 1898, suppl., par. 504.

Dubec, Presse Médicale, July 1, 1898.

Ellis, New York Med. Jour., Mar. 12, 1910.

Eppinger, Prag. Med. Woch., 1881, pp. 51, 52.

Fenwick, Soltau, Cancer of the Stomach, 1902, p. 313.

Flexner, S., Amer. Jour. of the Med. Sciences, Oct. 1898.

Lancereaux, Traité historique et pratique de la syphilis, 1873, p. 249.

Musser, Philadelph. Hosp. Reports, 1890, i. p. 170.

Simmonds, M., Med. Review, 1900, p. 463. 


\section{CHAPTER XXIV}

\section{GASTRIC ATONY AND GASTROPTOSIS}

IN gastric atony there is impaired contractile power of the muscular tone of the stomach, so that the stomach becomes dilated, and unable to expel its contents within the usual period after taking a meal.

Causes.-The most frequent causes are: (1) over-distension of the stomach with large meals, (2) too frequent meals, (3) over-indulgence in strong tea, and (4) the extra strain thrown on the stomach by a purely vegetarian diet. Malnutrition, insufficient exercise, a sedentary life in badlyventilated rooms, and prolonged use of drugs such as iodide of potassium, quinine, and arsenic, are also predisposing causes.

Gastric atony may be secondary to such diseases as anæmia, disease of the heart and liver, and chronic gastritis. Last, but not least, gastric atony is an invariable accompaniment of chronic constipation.

Symptoms.-Discomfort, and sense of weight or fullness, coming on immediately after food, and lasting for two or more hours, is the prominent symptom. The discomfort is referred to the epigastrium, and is accompanied by distension, so that often it is necessary to loosen the clothes. Belching of gas is a constant and troublesome feature of the condition, often forming the subject of the patient's chief complaint. Fluids generally cause more discomfort than solids. Constipation is always present. Dr. Soltau Fenwick has pointed out the significance of the presence of a stomach 
splash as indicating the existence of impairment of the gastric motility. "In any case," he writes, " of myasthenia, whether primary or secondary, clapitage may be observed from the commencement of the meal until the expiration of three or more hours, and, in severe instances, it may be only in the early morning that manipulation of the epigastrium fails to produce the characteristic sound."

Another test for atony is as follows: if, on an empty stomach, the patient take a wine-glassful of water, and splashing can be elicited, we may conclude that gastric atony is present.

Gastric Analysis.-The amount recovered after a testmeal is increased : instead of amounting to 150 c.c. it may be as much as 250 c.c. or more. In the later stages of the disease, food may be found in the stomach in the early morning.

The gastric contents are sour-smelling, but the chlorides do not appreciably deviate from the limits of health. When, however, the trouble is of old standing, or chronic gastritis is present, free hydrochloric acid may be absent, and the protein hydrochloric acid may be diminished. The condition must be distinguished from that of pyloric stenosis. In the latter disease the patient is more comfortable on a liquid diet, while in gastric atony a carefully-regulated solid diet gives more relief. In pyloric stenosis vomiting is frequent and severe, and malnutrition of the patient is pronounced.

Treatment.-The diet must be regulated carefully. Sweet or fatty foods and the amount of fluid taken must be limited strictly. Unless there be gastric catarrh, lavage is not indicated, but massage often is very helpful. Drugs are not of much service, with the exception of dilute hydrochloric acid when there is deficiency of gastric juice. Gastro-jejunostomy is contra-indicated. It is of no use in gastric atony. 
The most important symptom to be treated is constipation. The best drug is liquid paraffin, an ounce being given the last thing at night and a similar quantity in the early morning.

Although surgery rarely is indicated in the treatment of gastric atony, we must bear in mind that constipation may be the primary condition, and that constipation may be due to the presence of one of the kinks described by Mr. Arbuthnot Lane. The existence of these kinks has been questioned by some authorities, but as to their presence and injurious effects, I have no doubt whatever. These kinks can be demonstrated by means of the X-rays. I know from experience of a number of cases, that the division of them, or their elimination, by means of a short circuit between the ileum and pelvic colon, is followed by most gratifying results.

\section{GASTROPTOSIS}

Gastroptosis, or downward displacement of the whole stomach, rarely exists except in association with dislocation of other abdominal organs. The condition is often termed Glénard's disease, after the physician who was the first to describe it.

The lengthening and stretching of the ligaments and mesentery of the stomach may be so great, that the lower curvature may reach almost to the pubes, while the upper curvature may be as low as the level of the umbilicus.

Gastroptosis must be distinguished from dilatation of the stomach. In dilatation, the distance between the upper and lower borders of the stomach is much greater than normal, while in gastroptosis this distance is increased little, if at all. Dilatation, with atony of the stomach, may be present in addition to the displacement. 
The limits of the stomach are best determined by inflating the stomach with air, either by means of a stomachtube, or by administering separately, the two halves of a seidlitz powder.

Two Varieties of Gastroptosis are described, the congenital and the acquired.

1. Congenital Gastroptosis.-Usually this is accompanied by a general enteroptosis. Spiller, who has devoted particular attention to the condition, speaks of "an universal asthenia of a congenital nature, or habitus enteroptoticus." Patients thus affected have a stooping carriage, protuberant, lax abdomens, flat chests, and dyspeptic symptoms. Usually they are females in the early adult period of life.

2. Acquired Gastroptosis.-This is due to mechanical causes, such as frequent pregnancies, with insufficient rest after parturition, tight-lacing and other improper modes of dress, and to malnutrition, caused by insufficient, or unsuitable food.

Symptoms. - Patients complain of a sense of weight in the abdomen, fullness and discomfort after meals, sometimes nausea, and occasionally vomiting. Constipation is always present, sometimes of a very obstinate type.

The gastric symptoms are due to impaired motility, or to disordered secretion, or to both. The muscular tone of the stomach is impaired, there is delay in the passage onwards of the gastric contents. The stomach performs its work efficiently as to final results, but inefficiently as to time: it empties itself slowly, but completely, so that there is never stagnation, as is the case with pyloric stenosis.

The secretion of the stomach is increased, so that as a rule, gastric juice is present in the fasting stomach, and usually there is some hyper-acidity.

Treatment.-The treatment of gastroptosis is not surgical. In itself it is not a disease, many patients having marked 
gastroptosis, unaccompanied by any symptoms referable to the stomach. Symptoms, when present, are due to the want of tone of the muscular tissue of the abdominal wall and viscera, and to the general condition of the patient, and not to the displacement of the stomach.

Therefore I do not believe in performing gastropexy, or other operation for gastroptosis. I regard the various "pexies" as among the most unsatisfactory operations in surgery.

Moreover, the patients are generally of a type in which any surgical procedure should be avoided, if possible. They are only too ready to submit to operation, until, as Dr. W. J. Mayo puts it, " they have had all the movable organs fixed, and the removable ones removed." They rarely are benefited, and nothing results but failure and discredit.

The Treatment of Gastroptosis is twofold : first, general, and secondly, mechanical.

General.-Our aim should be to improve the general health of the patient. Abdominal massage is of service, and in some cases a rest cure is indicated. Hyper-secretion is treated by the administration of plenty of fat in the food, and perhaps the most important of all, constipation must be relieved. This is best effected by the administration, night and morning, of an ounce of pure liquid paraffin. Needless to say, the diet must be of the plainest description, starchy foods being rigorously limited.

Mechanical.-A well-fitting abdominal belt gives great comfort to the patient. It should fit firmly under the lower abdomen, and be kept in place, either by perineal straps, or by being made to fit round the upper part of the thighs. An aluminium plate on the lower part of the abdomen, moulded to fit accurately, adds to its efficacy. Such a belt gives relief, not by any pushing up of the displaced viscera, but by diminishing the amount of blood in the splanchnic area, and so raising the general blood-pressure. 


\section{APPENDIX}

\section{THE USE OF THE STOMACH-TUBE}

THE stomach-tube is made of soft red rubber with two lateral, oval openings near its tip. The tip should be solid, so that material does not collect below the lateral openings. The edges of the openings should be rounded, so that they

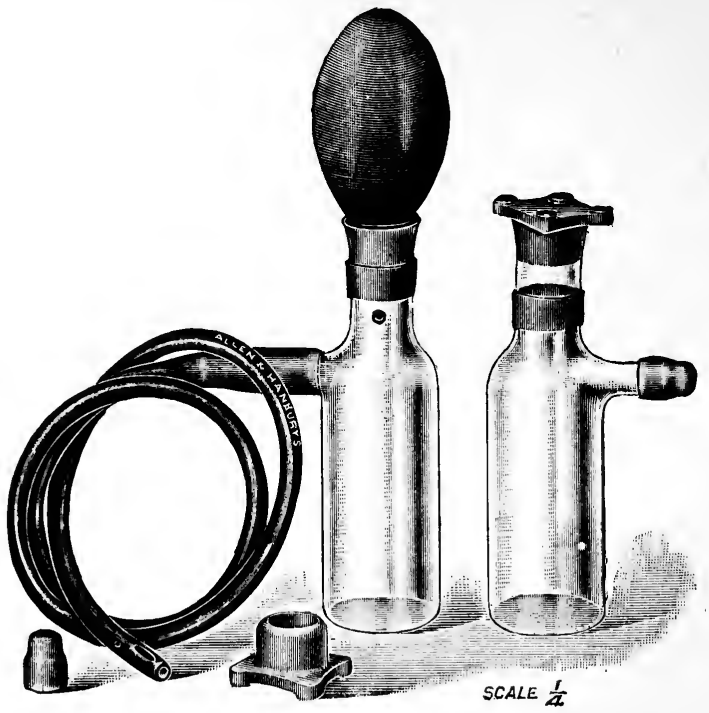

Frg. 74.-Senoran's stomach evacuator.

may not injure the gastric mucous membrane. As large a tube as possible should be used, as the larger the tube, the easier it is to pass, and the better it is tolerated. For adults a No. 32 or 36 (French scale) tube is a suitable size. Before use the tube is placed in hot water. No other lubricant is necessary. The patient is seated in a chair, with the head 
inclined slightly forwards. Any artificial teeth are removed. The tube is inserted into the mouth, and as soon as it touches the posterior pharyngeal wall the patient is directed to swallow, and the tube is pushed rapidly onwards until it reaches the stomach. If the patient be instructed to breathe rapidly through the nose, his attention is directed away from the unpleasantness of the tube, and it causes less discomfort than would otherwise be the case. The best method of withdrawing the stomach contents is by means of a Senoran's aspirator (Fig. 74). This consists of a glass bottle with a capacity of 200 c.c., to the mouth of which is fitted a compressible rubber bulb. From the neck of the bottle projects a nozzle, to which the stomach-tube can be attached. On the opposite side of the neck is a small hole. If the bulb be compressed, and the hole in the neck covered by the finger tip, a partial vacuum is produced in the bottle, and the stomach contents are sucked out.

\section{THE METHOD OF PERFORMING GASTRIC LAVAGE}

This is best carried out with Senoran's aspirator. The stomach-tube is passed and attached to the bottle. The bottle is filled with warm water. The finger is then pressed on the hole in the neck and the bulb is compressed. This drives the water from the bottle into the stomach. Then, by using the bottle as an aspirator, the fluid can be withdrawn from the stomach into the bottle. The bottle is emptied and refilled with warm water, which in turn is run into the stomach. The process is repeated until the water returned is clear.

\section{THE METHOD OF INVESTIGATING THE MOTOR FUNCTIONS OF THE STOMACH}

One method of investigating the motor functions is given in Chapter I. (p. 6). Another simple method, but less instruc- 
tive, is that of Ewald. The procedure is as follows : Fifteen grains of salol are given with the food, and the urine tested for salicylic acid at intervals. Salol is split up by the alkaline pancreatic juice, but it is not acted on by the gastric juices. Therefore, salicylic acid cannot appear in the urine until the salol has reached the duodenum. Normally, after the administration of salol, salicylic acid appears in the urine after an interval varying from forty to seventy minutes; in cases of motor insufficiency the acid will not be found until after a longer interval.

The test for salicyluric acid is made by putting a drop of urine on a filter paper, and then applying a drop of ferric chloride (10 per cent. solution) to the moist spot. If salicyluric acid be present a violet coloration will appear at the edge of the moist spot.

\section{QUALITATIVE EXAMINATION OF GASTRIC CONTENTS}

Reaction.-The reaction of the filtered gastric contents is tested by means of litmus paper. Normally the reaction should be acid, but it may be alkaline or amphoteric. If the reaction be acid, the filtered contents are tested for the presence of free hydrochloric acid. For this purpose one of several reagents may be used.

Congo Red.-A solution is prepared by dissolving one gramme of congo in 100 c.c. of distilled water. This solution turns blue on the addition of free hydrochloric acid. It is said that free hydrochloric acid, present to the amount only of 0.0009 per cent., is detected by this reagent.

It is more convenient to use red congo paper. This is made by saturating filter paper with an alkaline solution of congo, and allowing the paper to dry. Then it may be kept for use as required. The paper turns blue on the addition of filtered gastric contents containing free hydrochloric acid. 
It is a less delicate test than the solution, as the blue coloration does not appear unless 0.01 per cent. of free hydrochloric acid be present in the gastric contents. Inasmuch as free lactic acid or acetic acid turn congo paper blue, occasionally the reaction for free hydrochloric acid is present, although by quantitative analysis, free hydrochloric acid is proved to be absent.

Dimethyl-amido-azo-benzol Test for Free $\mathrm{HCl}$.-This test consists of a 0.5 per cent. alcoholic solution of dimethylamido-azo-benzol. A few drops of the reagent are placed in a test-tube or porcelain dish, and a few cubic centimetres of filtered gastric contents are added. If free hydrochloric acid be present the solution turns a cherry red. Organic acids, if present in a greater percentage than 0.5 per cent., will give the cherry colour.

Gunzberg's Test for Free HCl.-Gunzberg's solution is made up as follows :

$\begin{array}{llllll}\text { Phloroglucin } & \cdot & . & . & . & 2 \text { grammes } \\ \text { Vanillini } & \cdot & \cdot & \cdot & . & 1\end{array}$

This solution must be freshly prepared. If, however, the two reagents be made up separately, they may be kept for some months, and mixed together for use when required. To make the test, three drops of filtered gastric contents are placed in a porcelain capsule, and a few drops of the reagent added. The capsule then is heated very gently over a spirit flame. If free hydrochloric acid be present, as the fluid evaporates a beautiful cherry red appears round the edges of the mixture. This test is a very delicate and reliable one. Solutions of free hydrochloric acid as weak as 0.01 per cent. give the reaction. No organic acids give the reaction.

Test for Lactic Acid.-The presence of lactic acid is detected by Uffelmann's reagent. Ten cubic centimetres, of a one in twenty solution of carbolic acid, are placed in a 
test-tube, and a few drops of liquor ferri perchloridi added; this mixture is a deep amethyst blue. Enough distilled water is added to render the fluid transparent, and 5 c.c. of the resulting solution are placed in a test-tube. To this a few drops of filtered gastric contents are added. If lactic acid be present, a canary yellow or greenish-yellow colour results.

It is more delicate to make the test for lactic acid, after extracting the gastric contents, with ether. The ether dissolves the lactic acid, other substances being left behind. The ethereal solution is evaporated on a water bath, and the residue extracted with water and tested as described above. In the presence of lactic acid the solution turns green.

Strauss' Test.-A more delicate test is that of Strauss. If made with a special Strauss funnel the test is a very simple one. The funnel has a stop-cock at its lower end. Above the stop-cock two marks are engraved on the glass, one mark 5 c.c. and the other mark 25 c.c. above the stopcock. At the upper end of the funnel is a glass stopper. To apply the test, filtered gastric contents are run into the funnel up to the 5 c.c. mark, and ether added up to the 25 c.c. mark. The glass stopper is replaced and the funnel is shaken vigorously. It is then allowed to stand until the ether has separated from the gastric contents. The stopcock then is opened and the fluid allowed to run out slowly until the remaining fluid reaches to the 5 c.c. mark. The funnel is now filled up to the 25 c.c. mark with distilled water, and a few drops of tincture of iron chloride added. If lactic acid be present, the mixture, on being shaken, turns a deep or pale green, according to the amount of acid present.

Gmelin's Test for Bile Pigment.-Ten cubic centimetres of fuming nitric acid are placed in a test-tube. By means of a pipette an equal quantity of filtered gastric contents is floated on the nitric acid. If bile pigment be present, a 
green ring is formed at the junction of the nitric acid with the gastric contents.

The colour becomes more marked, if the test-tube be put aside for five or ten minutes.

Carmine Red Fibrin Test for Pepsin.-This method is described by. Harley and Goodbody as follows :

"Some fibrin from bullock's blood is quickly washed free from all traces of colouring-matter, this being important so as to avoid decomposition of the fibrin. The fibrin is then dried with alcohol and ether, and stained with an alcoholic solution of carmine-red until the colour does not deepen. Any excess of carmine is removed by washing in water, and the fibrin thus prepared is kept in glycerine.

Method of carrying out the Test.-Three test-tubes are prepared as follows :

(1) Four c.c. of filtered gastric contents.

(2) Two c.c. of filtered gastric contents and two c.c. of a four per mille hydrochloric acid solution.

(3) Two c.c. of filtered gastric contents and two c.c. of distilled water.

A small piece of carmin fibrin, which has been washed clear of glycerine, is placed in each of the test-tubes, which are then placed in a water bath at $37^{\circ} \mathrm{C}$., being taken out and shaken every two or three minutes. The time when the fluid is first coloured by carmine is noted. Pepsin, in the presence of hydrochloric acid, dissolves the fibrin, converting it into soluble fibrin, thus liberating the carmine which becomes diffused through the fluid, and indicates that digestion has commenced.

If pepsin and hydrochloric acid are present in the gastric contents in normal amounts, it will be found that test-tube No. 1 will show a red coloration in from three to five minutes. In the event of the hydrochloric acid in the gastric contents being normal, the additional quantity of hydro- 
chloric acid in test-tube No. 2 will tend to make it excessive, and thus hinder digestion.

In hypochlorhydria, or in cases where the proenzyme and not pepsin is present, test-tube No. 2 will show a red coloration before the other test-tubes.

In hyperchlorhydria, it will be found that test-tube No. 3, which has been diluted with water, will show distinct digestion of the fibrin before test-tubes Nos. 1 and 2, since the pepsin will not act as rapidly if hydrochloric acid is in excess in the gastric contents.

As examples of these three conditions, we will give three typical cases.

(A) Euchlorhydria

(1) Red coloration in 3 to 5 minutes.

(2) Red coloration in 3 to 5 minutes.

(3) Red coloration in 5 to 10 minutes.

(B) Hyperchlorhydria

(1) Red coloration in 7 to 15 minutes.

(2) Red coloration in 3 to 7 minutes.

(3) Red coloration in 3 to 12 minutes.

(C) Hypochlorhydria

(1) Red coloration in 10 to 30 minutes.

(2) Red coloration in 5 to 30 minutes.

(3) Red coloration in 30 to 120 minutes.

\section{QUANTITATIVE EXAMINATION OF GASTRIC CONTENTS}

Total Acidity.-The usual method of estimating the total acidity of the gastric contents is that introduced by Ewald (Klinik der Verdauungs Krankheiten, 1893, ii. p. 34).

Method.-Ten cubic centimetres of gastric contents are measured carefully into an Erlenmeyer's flask or small 
beaker. An equal quantity of distilled water is added, as well as a few drops of a one per cent. alcoholic solution of phenophthalein, to act as an indicator. From a burette a decinormal solution of sodium hydroxide is run in drop by drop, until the red colour, which the liquid in the flask gradually assumes, does not deepen on the addition of another drop. The number of cubic centimetres of sodium hydroxide used represents the total acidity in 10 c.c. of the filtered gastric contents. This figure multiplied by ten gives a number which expresses what is known as the total acidity. This number represents the acidity due to hydrochloric acid, plus organic acids, plus acid phosphates, in 100 c.c. of filtered gastric contents. In health it varies from fifty-five to sixty-five.

Estimation of Volatile Acids.-The simplest and best method is that devised by Professor Vaughan Harley (Brit. Med. Jour., 1899, ii. p. 1271). The method is as follows :

"A certain quantity (preferably 25 c.c.) of unfiltered gastric contents is poured into a distillation flask, and 100 c.c. or more of water added. A current of superheated steam is driven through the flask, and a small flame placed underneath it. The superheated steam, raising the contents to boiling-point, drives off the volatile acids, which distil over a condenser into an Erlenmeyer containing about 10 c.c. of decinormal solution of sodium hydrate. After about thirty minutes, the Erlenmeyer flask is removed, and a few drops of neutral solution of litmus added to the sodium hydrate solution. The fluid is then titrated by rapidly running into it a decinormal solution of sulphuric acid, until a neutral reaction is obtained. The quantity of decinormal solution of sodium hydrate neutralised by the volatile acids is then easily obtained. Multiplying the number by four, if 25 c.c. of gastric contents were taken, will give the quantity of volatile acids in 100 c.c. of gastric contents." 


\section{QUANTITATIVE ESTIMATION OF HYDRO- CHLORIC ACID}

Prout-Wynter Method.-This method is very accurate, but requires considerable practice to obtain reliable results. It is carried out as follows :

I. Place 10 c.c. of unfiltered gastric contents in a platinum capsule, and add 10 c.c. of a saturated solution of sodium carbonate; heat to dryness on a sand bath, taking care that the contents of the capsule do not spurt; then heat to redness, and, with distilled water, wash the contents of the capsule into a glass beaker, which should have a spout. Add sufficient nitric acid (about 10 c.c.) to neutralise the excess of sodium carbonate. Add 15 c.c. of silver nitrate (decinormal solution) from a burette, then filter into a larger beaker, being careful to wash the precipitate well with distilled water, at least three times. To the filtrate add a few drops of a saturated solution of iron alum, in distilled water, to act as an indicator. Next, slowly run in, from a burette, a decinormal solution of potassium sulphocyanide until the contents of the beaker have a faint, pinkish-yellow tinge. Subtract the number of c.c. of potassium sulpho-cyanide used from 15 c.c. (the amount of silver nitrate used), and this will give the number of c.c. of silver nitrate required to combine with the total chlorides in 10 c.c. of gastric contents. Multiply this number by 0.0365 , and the result will be the amount of total chlorides in 100 c.c. of gastric contents.

2. Place 10 c.c. of unfiltered gastric contents in a platinum capsule, evaporate to dryness so as to drive off the free hydrochloric acid, being careful that the contents of the capsule do not become charred. Add 10 c.c. of a saturated solution of sodium carbonate and proceed in exactly the same manner as in No. 1. 
3. Evaporate to dryness (in a platinum capsule) 10 c.c. of unfiltered gastric contents, heat to redness, and then proceed in exactly the same manner as in Nos. 1 and 2, except that only 10 c.c. of silver nitrate need be added, consequently the number of c.c. of potassium sulpho-cyanide used must be subtracted from ten instead of from fifteen.

I. The result of No. 1 gives the amount of total chlorides in 100 c.c. of unfiltered gastric contents.

2. The result of No. 2 gives the amount of all chlorides, except the free hydrochloric acid, in 100 c.c. of unfiltered gastric contents.

3. The result of No. 3 gives the amount of mineral chlorides only, in 100 c.c. of unfiltered gastric contents. Therefore :

No. $1=$ total chlorides.

No. 1 -No. 2 =free hydrochloric acid.

No. 3 -No. 2 =protein hydrochloric acid.

No. 3 =mineral chlorides.

Töpfer's Method.-This method is much simpler than the Prout-Wynter method, but it is not nearly so accurate. Personally, I do not consider that it is of much value.

Method.-I. 10 c.c. of filtered gastric contents are placed in an Erlenmeyer's flask, and a few drops of a 1.0 per cent. alcoholic solution of phenopthalein added. The contents of the flask then are titrated with a decinormal solution of sodium hydroxide in the way described above for the estimation of the total acidity. The number of c.c. of sodium hydroxide used is multiplied by ten. Let $\mathrm{A}$ represent this figure.

2. Another 10 c.c. of filtered gastric contents are placed in a flask, and a few drops of a watery solution of alizarin added. On the addition of alizarin the contents of the flask turn yellow. The contents then are titrated with a decinormal solution of sodium hydroxide until the yellow 
colour changes to violet. The number of c.c. of sodium hydroxide used is multiplied by 10 . Let $\mathbf{B}$ represent this figure.

Then, A-B represents the amount of protein hydrochloric acid.

3. Another 10 c.c. of filtered gastric contents are measured into a flask and a few drops of dimethyl-amido-azo-benzol solution $(0.5$ per cent. in alcohol) are added. The mixture is titrated as before. The number of c.c. of sodium hydroxide used is read off and multiplied by 10 . This gives the acidity due to free hydrochloric acid. Let $\mathbf{C}$ represent this figure.

As I pointed out in the description of the method of estimating the total acidity, the figure obtained represents the acidity due to hydrochloric acid, organic acids, and acid phosphates. Therefore:

A represents the total acidity due to hydrochloric acid, organic acids, and acid phosphates.

C represents the acidity due to free hydrochloric acid.

A-B represents the acidity due to protein hydrochloric acid.

A- $(B+C)$ represents the acidity due to organic acids and acid phosphates.

\section{QUANTITATIVE ESTIMATION OF PEPSIN}

Mett's Method (Pawlow, The Work of the Digestive Glands, translated by W. H. Thomson, 1902, p. 26). Some fresh egg albumen is drawn into a fine capillary glass tube. The tube is placed in boiling water for five minutes. This coagulates the albumen. The tube and its contents are then cut into lengths measuring five centimetres each. To estimate the pepsin in the gastric contents, proceed as follows :

Some gastric contents (unfiltered) are placed in a testtube or small beaker, and a piece of the prepared tube placed therein. The beaker then is placed in an incubator at a temperature of $37^{\circ} \mathrm{C}$. for ten hours. At the end of 
this time, if the capillary tube be examined, it will be seen that some of the albumen has disappeared from each end of the tube. The empty portions of the tube are measured by means of a millimetre scale and a magnifying glass. The square of the length of the column of albumen digested is the measure of the amount of pepsin in the gastric contents. For example, if the empty portion of the capillary tube measure two millimetres in length, the amount of pepsin present is represented by $2 \times 2$, that is, four parts of pepsin.

The unit is the quantity of pepsin which will digest one millimetre of egg albumen in a Mett's tube in ten hours, the tubes being immersed in 0.18 per cent. of free hydrochloric acid.

\section{TEST FOR OCCULT BLOOD IN THE FACES}

A piece of fæces the size of a hazel nut is mixed with water in a test-tube, so as to form a thick emulsion; to this one-third of its volume of glacial acetic acid is added and the mixture shaken. Then 5 c.c. of ether are added and the two fluids are mixed by being shaken. On standing, the ethereal extract separates, and of this 2 c.c. are mixed with 2 c.c. of a saturated solution of benzidin (para-diamido-diphenyl) in rectified spirit, and 2 c.c. of a solution of hydrogen peroxide. If blood be present in considerable quantity, the solution assumes immediately a deep-blue colour; in lesser quantities the colour is less intense and green.

For three days before the test is applied the patient's diet must be free from blood-containing foods.

\section{THE SERO-DIAGNOSIS OF CANCER}

Draw 15 grammes of blood. It must not be more than thirty-six hours old or forty-eight at most. To $0 \cdot 1$ c.c. of the serum in a test-tube, 1 c.c. is added of a 5 per cent. suspension of hen's blood corpuscles, in a 0.85 per cent. 
solution of sodium chloride. The test-tube is well shaken and placed in a water bath, and this in an incubator at a temperature of $37^{\circ} \mathrm{C}$. A positive reaction consists in the entire absence of hæmolysis in the test-tube.

Kelling (Archiv für Verdauungs Krankheiten, Berlin, xvi. ii. No. 3; also Jour. Amer. Med. Assoc., 1906, xlvii. p. 1963 ; 1910, iv. p. 1602) attaches great value to the sero-diagnosis of early cancer. He states that from an experience of the test in 1500 patients, 400 of whom had cancer, a positive reaction was never obtained in a healthy person. He maintains that a negative reaction excludes cancer. Rosenberg (Deutsche Mediz. Woch., Berlin, June 27, vol. xxxvii. No. 26) finds that a positive reaction is found in many conditions other than cancer, and he is of opinion that the test is of little practical differential value.

\section{THE SKIN REACTION IN CARCINOMA}

Elsberg and Geist (Amer. Jour. of the Med. Sciences, Feb. 1910) have suggested a new method for detecting hæmolysis in patients suffering from cancer. Their method is to inject, subcutaneously, into the arm of the patient suspected to have cancer, five minims of a suspension of 20 per cent. washed human blood corpuscles in saline solution.

The corpuscles are attacked by the hæmolysins in the blood serum of the cancer patient, and a reaction appears in from two to eight hours. The reaction is the appearance, at the site of the injection, of a brownish-red or bluish colour.

It is said that the reaction occurs in nearly 90 per cent. of cancer cases.

\section{THE LENHARTZ DIET IN THE TREATMENT OF GASTRIC ULCER}

Professor Lenhartz keeps his patients at absolute rest, and even after hæmorrhage commences to feed them at once 
on a diet of raw eggs and milk. On the first day he gives 100 c.c. ( $3 \frac{1}{2}$ ounces) of iced milk, in which is beaten up one raw egg. The mixture is sipped slowly out of a teaspoon, so that the patient takes a continuous supply of protein to combine with the hydrochloric acid of the gastric juice, and thus diminish any hyper-acidity which may be present. The quantity taken is increased day by day until it amounts to 800 c.c. ( $28 \frac{1}{2}$ ounces) of milk and six or eight eggs a day.

It is maintained that this diet contains a minimum amount of food with a maximum calorific value, so that by its use the patient's general nutrition is improved, and the healing of the ulcer promoted. Further, the continual presence, in the stomach, of protein food "fixes" the free hydrochloric acid, and thus diminishes the hyper-acidity which retards healing of the ulcer.

The diet is an excellent one, but personally, I do not approve of commencing it at once after gastric hæmorrhage. On several occasions I have seen an alarming hæmorrhage occur in a patient put on this regimen immediately after hæmatemesis. After hæmorrhage, therefore, I prefer to withhold all food by the mouth, and to give saline by the rectum, for three days. If, at the end of this period, the hæmorrhage have ceased, the Lenhartz diet may be commenced with safety.

\section{SCHMIDT'S TEST DIET}

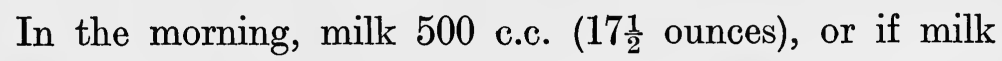
does not agree, 500 c.c. of cocoa made from cocoa powder, 20 grammes ( $\frac{2}{3}$ of an ounce); sugar 10 grammes ( $\frac{1}{3}$ of an ounce); water 400 grammes (14 ounces); and milk 100 grammes ( $3 \frac{1}{2}$ ounces). In addition to the milk or cocoa 50 grammes of zweiback (six biscuits may be substituted for the zweiback). 
In the forenoon, 500 c.c. ( $17 \frac{1}{2}$ ounces) of oatmeal gruel (made from oatmeal $1 \frac{1}{2}$ ounces, butter $\frac{1}{3}$ of an ounce, milk

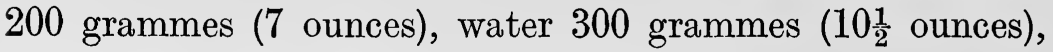
and one egg).

At noon, 125 grammes ( $4 \frac{1}{3}$ ounces) raw weight of chopped beef broiled rare with 20 grammes ( $\frac{2}{3}$ of an ounce) of butter, so that the interior still remains raw. In addition to the beef, 250 grammes of potato purée made from mashed potatoes 190 grammes ( $6 \frac{2}{3}$ ounces), 100 grammes of milk ( $3 \frac{1}{2}$ ounces), and 10 grammes ( $\frac{1}{3}$ of an ounce) of butter.

In the afternoon, as in the morning.

In the evening, as in the forenoon.

This diet contains 102 grammes of albumin, 111 grammes of fat, 191 grammes of carbo-hydrates, a total of 2234 calories.

According to Schmidt, the chief inferences which may be drawn from examination of the fæces of a patient on this diet are the following :

r. The appearance of mucus in the fæces indicates the existence of an inflammatory condition of the mucous membrane.

2. If connective tissue appear in the fæces it is a sign of disturbance of the stomach digestion.

3. If macroscopic muscle remains appear in the fæces, this is a sign of a disturbance of intestinal digestion.

4. Remains both of connective tissue and of muscle, indicate disturbances in which stomach and small intestine participate.

5. Complete absence of the red and green colouring of fæces, when tested with mercuric chloride, occurs in fatty stools when the bile is completely shut off from the intestine. 


\section{LIST OF AU'THORI'TIES}

Agnes, Sister, 61

Allbrecht, 79

Anchütz, 83

Andral, 262

Aschoff, 262

Ashurst, 266

BARBAT, 103

Bardsley, H., 254

Batten, F. E., 257

Baumberger, 202

Bendix, 257

Beyea, 55

Billroth, 185

Bloch, 257

Boas, 10, 222

Bolton, C., 117

Bouveret, 204

Box, C. R., 79

Braun, 95

Bret, 262

Brinton, 208, 212, 262

Bull, 24

Burghard, F., 24, 260

CAIRd, F. M., 203

Cameron, H. C., 92

Cantwell, 267

Carle, 83, 90

Carrington, 194

Cartwell, 232

Carvalho, 89, 241

Cautley, E., 254, 260

Chiari, 180

Chrostek, 203

Coffey, R. C., 55

Cornil, 207

Crile, G. W., 72

Cruveilhier, 180, 248, 262

Cumston, 195

Cuneo, 209, 212

Cushing, H., 18

Czerny, 212

\section{DASTRe, 87}

Daworsky, 254

Deaver, J. B., 224, 266, 270

Dent, C. T., 254, 260

D'Espere, 206

Devic, 204

Dieulafoy, 115, 180, 183

Dittrich, 248
Doyen, 142, 195

Dujardin-Beaumetz, 202

Duret, 55

EHRET, 222

Eiselsberg, von, 78, 266, 267

Elsberg and Geist, 302

Erb, 203

Erlach, von, 266

Eve, F., 55

Ewald, 8, 270, 292, 296

Fantino, 83, 90

Fenwick, Soltau, 77, 181, 203, 206, 207, $208,215,222,231,248,266,274,277$, 281,283

Feuerer, G., 47

Finney, J. M. T., 27

Foote, 174, 200

Fox, W., 262

GaRdNer, BeLlamy, 76

Glénard, 287

Gould, A. H., 103, 104

Graff, von, 232

Graham, C., 136, 248

Gressot, 145, 253

HABERKANT, 43

Häberlin, 206, 248

Habershon, S., 208

Hacker, V., 267

Hahn, 267

Halsted, W. S., 242

Hammerschlag, 221

Handley, S., 244

Harley, V., 12, 297

Harley and Goodbody, 295

Harrington, 103

Hartmann, 43, 90

Hawkins, H. P., 169, 176

Hayem, 107

Hemmeter, 222

Henschell, 256

Herman, 267

Heubner, 257

Hill, W., 16

Hirsch, 194

Hirschsprung, 254

Hochenegg, 195

Hoffmann, 203

Hudson, 194

Hutchison, R., 256, 257 
JöSLIN, 92

KAUFFMAN, 222

Keen, 173,174

Kelling, 83, 302

Kocher, Th., 145, 253

Korte, 185

Krehl, 239

Kussmaul, 202

Lane, W. Arbuthnot, 20, 127, 131, 287

Latham, A., 267

Lebert, 248

Lenhartz, 108, 302

Leube, 149

Levy, 239

Löbker, 258

Loeb, 202

Lubarsch. 241

MacCarty, W. C., 211, 248

Massè, 87

Marten, 204

Matthieu, 150

Mauclaire, 81

Maurice, 158

Maylard, A., 267

Mayo, W. J., 28, 43, 53, 76, 81, 116, 128, $143,144,151,240,242,248,270,277$, 289

Mayo, C. H., 116, 240, 270

Meltzer, 258

Mett, 300

Meyer, W., 48

Mikulicz, 43, 95, 185, 239, 242

Monprofit, 105

Morgagni, 193, 266

Moullin, Mansell, 186

Moynihan, B. G. A., 41, 78, 87, 130, 132, $151,158,194,197,213$

Müller, 202

Murchison, 180

Murphy, J. B., 22, 64, 270

Murphy, F. T., 103

Noorden, von, 90

Nothnagel, 262

OCHSNER, 270

Ogston, 6

Osler, W., 254

PACHON, 89, 241

Pasteur, W., 76

Paviot, 262

Pawlow, 8, 88, 108'

Perry and Shaw, 231

Pilcher, L., 53

Plummer, H. S., 246

Powers, C. A., 78
RANVIER, 207

Riegel, 222

Riese, 242

Reuvers, 202

Robson, Mayo, 54, 194

Rodman, 52, 78

Rokitansky, 212

Rolleston, H. D., 256, 267

Rosenberg, 302

Rosenheim, 90, 248

Rotgans, 95

Roux, 39, 183, 264

Rovsing, 55

Royle, Elsie, 223

Rydygier, 90, 142, 183

Savariadd, 183

Schlatter, 89

Schlesinger, 222

Schmidt, 90, 303

Schostak, 95

Schwarz, 194

Schwyzer, 258

Senator, 270

Senn, 25

Senoran, 6, 290

Shattock, S. G., 263

Sievers, 194

Sonicksen, 248

Soupalt, 90

Spencer, W. G., 266

Spiller, 288

Starck, 257

Stiles, H. J., 32, 258

Still, G. F., 257

Strauss, 294

Symonds, C. J., 246

Talma, 142

Terrier, 81

Tinker, 174

Thomson, Alexis, 262

Thomson, Campbell, 79

Thomson, John, 254

Thursfield, H., 231

Töpfer, 299

Trousseau, 203

Tupolske, 45

UfFELmaNe, 11, 293

Wallace, C., 79

Weir, 174, 200

Welch, 154, 208

Wertheimer, 88

Wickenhauser, 95

Wilks, S., 262

Williams, R., 194, 254

Wilson, L. B., 248

Wölfler, 39, 197

ZENKER, 248 


\section{IN D EX}

Abdominal belt, 289 after operations, 70

Acidity, total, in health, 11 estimation of, 296

Acids, volatile, test for, 10 estimation of, 297

Acute dilatation of the stomach, 79

Acute perforation, 153, 164

Adeno-carcinoma, 207

Adenomata, 265

Analysis, gastric, 8, 292 typical, 14

Anterior gastro-jejunostomy, 30

Appendicular gastralgia, 270

free $\mathrm{HCl}$ in, 14, 272, 276

gastric analysis in, 12, 272

indications for operation in, 278

operation findings in, 273

pain in, 270

symptoms of, 270

Atony, gastric, 5, 285

tests for, 286

Auscultatory percussion, 6

\section{BACILLUS FILIFORMIS, 222}

Benign tumours of the stomach, 265 symptoms of, 267

treatment of, 267

varieties of, 265

Bile, in fasting stomach, 86

in stomach after gastro-jejunostomy, $80,83,86,104$

pigment, 10 test for, 294

Biliary colic, 3, 163

Bilious attacks after gastro-jejunostomy, 82

Blood, the, in carcinoma, 301

in fæces, 119, 182, 216 tests for, 301

Boas' test breakfast, 10, 222

Bowels after gastric operations, 17, 68

Cancer (see Carcinoma)

Carcinoma, sero-diagnosis of, 301

skin reaction in, 302

of body of stomach, 239

of cardiac end of stomach, 239

Carcinoma, gastric, 206

the blood in, 301
Carcinoma, gastric, diagnosis of, 224 differential diagnosis of, 227

free $\mathrm{HCl}$ in, 13, 219

frequency of, 206

gastrectomy for, 42, 239

gastric contents in, 218

grafting on ulcer, of, 248

hæmorrhage in, 207

lymphatic glands in, 209

pain in, 3, 215

palliative operations for, 242

partial gastrectomy for, 42,236

pathology of, 207

results of gastrectomy for, 241

spread of, 214

symptoms of, 214

treatment of, 236

urine in, 223

Cholelithiasis (see Gall-stones)

Chlorides, total, 12

in duodenal ulcer, 12, 138

estimation of, 298

in gastric carcinoma, 219

in health, 12

Chlorides, mineral, 14

after gastro-jejunostomy, 88

estimation of, 298

in gastric carcinoma, 219, 221

Clinical history, importance of, 1

in duodenal ulcer, 131

in gastric ulcer, 117

in perforation, 155

Closure of gastro-jejunostomy openings, 104

Coliac axis, glands round, in cancer, 210

Colic, biliary, 3, 163

Complications after gastric operations, 71 of the lungs, 76

Complications peculiar to gastro-jejunostomy, 80

" circulus vitiosus," or regurgitant vomiting, 80

diarrhœa, 83

internal hernia, 84

jejunal ulcer, 95

Complications of gastric ulcer, 153

of duodenal ulcer, 153

Congo red, test for free $\mathrm{HCl}, 292$

Continuous proctoclysis, 63

Cylindrical-celled carcinoma, 207 


\section{THE STOMACH}

Diagnosis of gastric ulcer, 123

of duodenal ulcer, 136

in perforation of gastric ulcer, 159

duodenal ulcer, 159

in gastric carcinoma, 224

sero-diagnosis of cancer, 301

Diarrhœa, post-operative, 83

Diet, after gastric operations, 66

Schmidt's, 303

results in fæces after Schmidt's, 304

Lenhartz's, 302

Differential diagnosis of gastric ulcer, 124,277

of gastroptosis, 287

of duodenal ulcer, 139, 276

of perforation of gastric ulcer, 161 duodenal ulcer, 161,198

of gastric carcinoma, 198, 227

of hour-glass stomach, 198, 227

Dilatation of the stomach, $5,191,287$

acute post-operative, 79

atonic, 5, 285

chronic, 191

diagnosis of, 192

treatment of, 192

Di-methyl-amido-azo-benzol test, 293

Drainage tubes, 69

after operation for perforated ulcer, 172

Duodenal ulcer, 128

acute, 128

ætiology of, 130

and appendicular disease, 130

acute, perforation of, 164

chronic, 128

chronic, perforation of, 165

clinical history of, 131

complications of, 153, 180, 191, 202

diagnosis of, 136

differential diagnosis of, 139

frequency of, 130

free $\mathrm{HCl}$ in, 135

gastric contents in, 135

hæmorrhage in, 134

operations for, 151

pain in, 2, 133

symptoms of, 133

treatment of, 149

medical treatment of, 150

surgical treatment of, 151

vomiting in, 133

Duodenum, hour-glass, 129

trifid, 129

carcinoma of, 213,252

EN-Y GASTRO-JEJUNOSTOMY, 39 objections to, 40,105

Erb's symptom in gastric tetany, 203

Erosions, 115

Ether, open method of administration,
Examination, physical, 5

of vomit, 7

Excision of gastric ulcers, 52 for hæmorrhage, 186

Exploratory gastrotomy, 23 incision, 16

Exulceratio simplex, 115

FACES, blood in, 119, 216 test for occult blood in, 301

Fat absorption, effect of gastro-jejunostomy, on, 92

Fibroma of stomach, 266

Fibromatosis of the stomach, 262 differentiation from cancer, 262 treatment of, 264

Free hydrochloric acid, 10 estimation of, 298

in health, 13

in various diseases, 10,13

tests for, 292

Fowler position, 61

Gall-Stones, pain in, 2 total acidity in, 12

Gastrectomy, 42 complete, 51

contra-indications, to, 238

mortality rate, 240

partial, 42

recurrence after, 241

results of, for malignant disease, $\mathbf{2 4 0}$

results, immediate and remote, 240

section of the stomach in partial, 43

Gastrectasis (see Dilatation)

Gastric analysis, 8

in appendicular disease, 272

in duodenal ulcer, 135

in gastric atony, 286

in gastric carcinoma, 218

in gastric ulcer, 120

in hour-glass stomach, 197

methods of, 292-5

Gastric atony, 5, 285

causes of, 285

gastric analysis in, 286

symptoms of, 285

treatment of, 286

Gastric carcinoma (see Carcinoma, gastric)

Gastric cases, method of investigation of, 1

Gastric contents, character of, 9

Gastric dilatation, 191

diagnosis of, 192

treatment of, 192

Gastric erosions, 115

Gastric functions, after gastrectomy, 241

Gastric hæmorrhage (see Hæmorrhage in Gastric and Duodenal Ulcers)

Gastric lavage, 291 after gastro-jejunostomy, $75,79,81$ 
Gastric lavage, for post-operative vomiting, 75, 79, 81

in acute dilatation of the stomach, 79

in cases of perforation, 172,173

methods of performing, 291

Gastric operations (see Operations)

Gastric sarcoma (see Sarcoma, gastric)

Gastric tetany (see Tetany, gastric)

Gastric ulcer, 115

acute perforation of, 153, 154

acute round, 115

ætiology of, 117

chronic, 116

chronic perforation of, 153, 176

clinical history of, 117

complications of, $153,180,191,193$, 202

diagnosis of, 123

differential diagnosis of, 124

gastric contents in, 120

excision of, 52

excision of, for hæmorrhage, 186

exulceratio simplex, 115

free $\mathrm{HCl}$ in, 13,121

hæmorrhage from, 119

Lenhartz's diet in, 302

mortality of surgical treatment of, 147

natural history of, 146

physical examination in, 120

simple, 115

symptoms of, 118

treatment of, 142

vomiting in, 119

Gastro-anastomosis, 42

Gastro-colic glands, 210

Gastro-enterostomy (see Gastro-jejunostomy)

Gastro-gastrostomy, 41

Gastro-jejunal ulcers (see Jejunal and Gastro-jejunal Ulcers)

Gastro-jejunostomy, 30

after-treatment, 61, 111

anterior, 30

for hæmorrhage, 188

complications peculiar to, 80

effect on fat absorption, 92

on gastric secretion, 87

digestion, 89

metabolism, 92

motility of the stomach, 90

nitrogen absorption, 92

En-Y, 39

hyper-acidity, after, 103, 106

openings, closure of, 104

physiological effects of, 86

posterior, 35

Roux's En-Y, 39

technique of, 30,110

Gastrolysis, 199
Gastropexy, 55

Gastro-plasty, 41

Gastroptosis, 5, 287

acquired, 288

congenital, 288

symptoms, 288

treatment, 288

Gastrorrhagia (see Hæmorrhage in Gastric Ulcer)

Gastroscope, the, 16

Gastrostomy, 25

Gastrotomy, 23

for foreign bodies, 23

for hæmorrhage, 25

Glands, lymphatic, in gastric cancer, 209

Glénard's disease (see Gastroptosis)

Gmelin's test for bile pigment, 294

Gummata, 281

Gunzberg's test, 293

Hematemesis (see also Hæmorrhage), 4 post-operative, 78

Hæmorrhage in gastric ulcer, 119, 180

in duodenal ulcer, 134,189

excision of ulcer for, 186

gastrotomy for, 25,188

treatment of, 183

treatment by gastro-jejunostomy, 186

treatment by Lenhartz's diet, 302

History in gastric cases, importance of, 1

Hour-glass duodenum, 129

Hour-glass stomach, 193

acquired, 194

cancerous, 239

congenital, 194

diagnosis of, 198

gastric analysis in, 197

pathology of, 194

prognosis of, 200

symptoms of, 196

total acidity in, 11, 198

treatment of, 199

Hunger-pain, 132

Hydrochloric acid, test for, 292, 298 (see also Free Hydrochloric Acid)

Hyper-acidity, distinction of from hyperchlorhydria, 11

cause of, after gastro-jejunostomy, 106

Hyper-chlorhydria, definition of, 11

in appendicular gastralgia, 272

in duodenal ulcer, 13, 135

Hyper-secretion, 3

in appendicular gastralgia, 7, 272

in duodenal ulcer, 135

in gastric ulcer, $3,7,120,124$

Hypertonic contraction in duodenal ulcer, 15

Hypertrophic pyloric stenosis, infantile, 254 
Hypertrophic pyloric stenosis, infantile, ætiology of, 255

choice of operation for, 259

morbid anatomy of, 254

symptoms of, 256

treatment of, -257

Incision, exploratory, 16

Incision, in gastric operations, 21

Infantile hypertrophic pyloric stenosis (see Hypertrophic, \&c.)

Internal hernia, 84

Intestinal stasis, signs of, 127

Investigation of gastric cases, method of, 1

JeJUNAL and gastro-jejunal ulcers, 95

ætiology of, 99

clinical groups of, 96

diagnosis of, 98

frequency of, 95

medical treatment of, 108

pathology of, 98

"peptic" hypothesis of, 100

risk of, 96,149

spontaneous healing of, 108

surgical treatment of, 109

swelling in, 97

symptoms of, 96

Jejunostomy, 53

Jejunum, ulcer of (see Jejunal Ulcer)

\section{LACTIC ACID, 10}

in gastric carcinoma, 221

in normal gastric contents, 10

test for, 293

Leather-bottle stomach, 208

Linitis plastica (see Fibromatosis)

Lipoma, 266

Loreta's operation, 24

Lung complications, post-operative, 76

Lymphadenoma, 266

Lymphatic glands, in gastric cancer, 209 of the stomach, 210

Medical treatment, of gastric ulcer, 149 duodenal ulcer, 150

jejunal and gastro-jejunal ulcer, 108

Mctabolism, effect of gastro-jejunostomy on, 92

Method of investigation of gastric cases, 1

Mett's method of estimating pepsin, 300

Mineral chlorides (see Chlorides, Mineral)

Motor functions of the stomach, 6 methods of investigation of, 6, 291

Myomata of stomach, 266

NEEDLE, " grip-eyed," 22

Nitrogen absorption, effect of gastrojejunostomy on, 92
Occult blood in fæces, test for, 301

Esophageal stricture, 6 as shown by X-rays, 15

cancerous, 25, 246

fibrous, 25

impermeable, 25

malignant, 25

Esophagus, removal of foreign bodies from, 24

Operation area, preparation of, 19

Operation, complications after, 71 for perforation, technique of, 167 preparation of patients for, 17 treatment of patients after, 61

Oppler-Boas bacillus, the, 222

PaIN, after gastric operations, 72 in duodenal ulcer, 133

gastric disease, 2, 118, 215

in appendicular gastralgia, 270

Palliative operations for gastric carcinoma, 242

Pancreatic juice in stomach after gastrojejunostomy, 87, 104

Pancreatitis, acute, 164

Parotitis, post-operative, 77

Partial gastrectomy, 42

Pepsin, estimation of, 300

Pepsin, test for, 295

"Peptic " hypothesis of jejunal ulcer, 100

Percussion, auscultatory, 6

Perforation of gastric ulcer, 153

acute, 153,154

chronic, 153

diagnosis of, 159

differential diagnosis of, 161

frequency of, 154

immediate results of operation for, 173

mortality rate of, 173

remote results of operation for, 175

sex incidence in, 154

signs of, 156

symptoms of, 156

site of, 154

technique of operation for, 167

treatment after operation for, 172

Perforation of duodenal ulcer, diagnosis of, 165

differential diagnosis of, 165

frequency of, 154

immediate results of operation for, 173

mortality rate of, 173

remote results of operation for, 175

sex incidence in, 154

signs of, 156, 165

symptoms of, 156

site of, 165

technique of operation for, 167

treatment after operation for, 172 


\section{INDEX}

Perigastric abscess, 154, 176 pus in vomit in, 7

Peritonitis, septic, 73

Phlebitis, post-operative, 78

Physical examination, 1, 5 general, 5 special, 5 in gastric ulcer, 120

Physiological effects of gastro-jejunostomy, 86

Pleurisy, post-operative, 76

Pneumonia, post-operative, 76

Polypi, of stomach, 265

Post-anæsthetic vomiting, 74

Post-operative complications, 71 bilious attacks, 83

diarrhœa, 83

dilatation of the stomach, 79

hæmatemesis, 78

internal hernia, 84

lung complications, 76

parotitis, 77

phlebitis, 78

pseudo-ileus, 75

shock, 71

septic peritonitis, 73

thrombosis, 78

vomiting, 74

Preparation of patients for gastric operations, 17

of operation area, 19

Proctoclysis, continuous, 63

Protein hydrochloric acid, estimation of, 298

in health, 14

in various diseases, 14

Prout-Wynter method of estimating $\mathrm{HCl}$, 298

Pseudo-ileus, 75

Pus in vomit, 7

Pyloric carcinoma, 208, 236 tumour in, 216

Pyloric stenosis (see Stenosis, pyloric)

Pyloro-gastro-duodenostomy, 27

Pylorodiosis (see Loreta's Operation)

Pyloroplasty, 27 results of, 27

Pylorus, dilatation of, 24

QUalitative examination of gastric contents, 292

reaction of gastric contents, 292

Quantitative examination of gastric contents, 296

Reaction, skin, in carcinoma, 302

Rectal feeding, 67

Regurgitation of bile after gastrojejunostomy, 86, 104

Regurgitant vomiting, 80
Resection of the stomach (see Gastrectomy)

Rest in bed after operation, 70

SARComa, gastric, 231 pathology of, 232 symptoms of, 232

Schmidt's diet, 303 results of, on fæces, 304

Septic peritonitis, 73

Senoran's aspirator, 290 for gastric lavage, 291

Sero-diagnosis of cancer, 301

Sequelæ of gastric ulcer (see Complications of)

Shock, causes of, 71 treatment of, 71

Skin reaction in carcinoma, 302

Special physical examination, 5

Spheroidal-celled carcinoma, 207

Stenosis, pyloric, 7, 13, 191

in carcinoma, 208, 217

infantile (see Hypertrophic), 254

with hour-glass stomach, 199, 200

in syphilis of the stomach, 282

treatment of, $53,192,200,245,257$ 282

Stomach, acute dilatation of, 79 adenoma of, 265

analysis of contents of, 292

atonic dilatation of, 285

benign tumours of, 265

bile in, after gastro-jejunostomy, 80 , 33, 86, 104

bi-locular (see Hour-glass)

cancer of body of, 239

cancer of cardiac end of, 239

chronic dilatation of, 191

congenital hour-glass, 194

fibromatosis of, 262

foreign bodies in, 23

functions of, 240

growths in, symptoms of, 267

treatment of, 267

lymphatics of, 209

motor functions of, 6

motility in duodenal ulcer, 7

polypi of, 265

removal of growths from, 24

sarcoma of, 231

syphilis of, 281

tubercle of, 283

ulcer of, 115

Stomach, hour-glass (see Hour-glass)

Strauss' test, 294

Stricture, œsophageal, 6, 15, 246

as shown by X-rays, 15

malignant, 25,246

inoperable fibrous, 25

Sub-acute perforation of gastric ulcer, 153

Sub-diaphragmatic abscess (see Sub. phrenic) 
Sub-phrenic abscess, 176 symptoms of, 177 treatment of, 178

Succussion splash, 5, 285

Swelling, in jejunal and gastro-jejunal ulcer, 97

Syphilis of the stomach, 281

chronic gastritis in, 281

gummata in, 281

pyloric stenosis in, 282

symptoms of, 283

treatment of, 283

Symonds' tube, use of, 246

Tecinique of operation, of gastrojejunostomy, 30, 110

for perforation of gastric or duodenal ulcer, 167

Test-meal, 9

Tetany, gastric, 202 symptoms of, special, 203

Thrombosis, 78

'Töpfer's method of gastric analysis, 299

Total acidity, 11 after gastro-jejunostomy, 87 estimation of, 296

in appendicular gastralgia, 272

in gastri; ulcer, 11,121

in duodenal ulcer, 11,138

Total chlorides (see Chlorides)

Transverse colon, in gastric carcinoma, $47,211,237$

Trifid stomach, 196

Treatment of, acute perforation, 166 duodenal ulcer, 149

gastric carcinoma, 236

gastric sarcoma, 236

gastric ulcer, 142

hour-glass stomach, 199

jejunal and gastro-jejunal ulcer, 107 patients after gastric operations, 61
Treatment of patients after operation for perforation, 172

pyloric stenosis, $53,192,200,245$, 257, 282

shock, 71, 72

wound, 69

Tubercle of the stomach, 283 primary, 283 surgical treatment, of, 283

Tumours, benign, of the stomach, 265 direction of growth of, 265 symptoms of, 267 treatment of, 267

ULCERS, malignant degeneration of, 143 248

Ulcer, gastric, 115 duodenal, 128 of the stomach (see Gastric)

Ulcus carcinomatosum, 248 clinical evidence of, 249 pathological evidence of, 251

Uffelmann's reagent, 293

Urine, in gastric carcinoma, 223

Volatile acids, 10 in various diseases, 12 estimation of, 297 test for, 10

Vomit, blood in, 7 examination of, 7 pus in, 7

Vomiting in gastric carcinoma, 217 in gastric ulcer, 119 duodenal ulcer, 133 in pyloric stenosis, 192 post-anæsthetic, 74

Wound, closure of, 21 treatment of, 69

Printed by Ballantyne, Hanson \&o Co.

Edinburgh \& London 



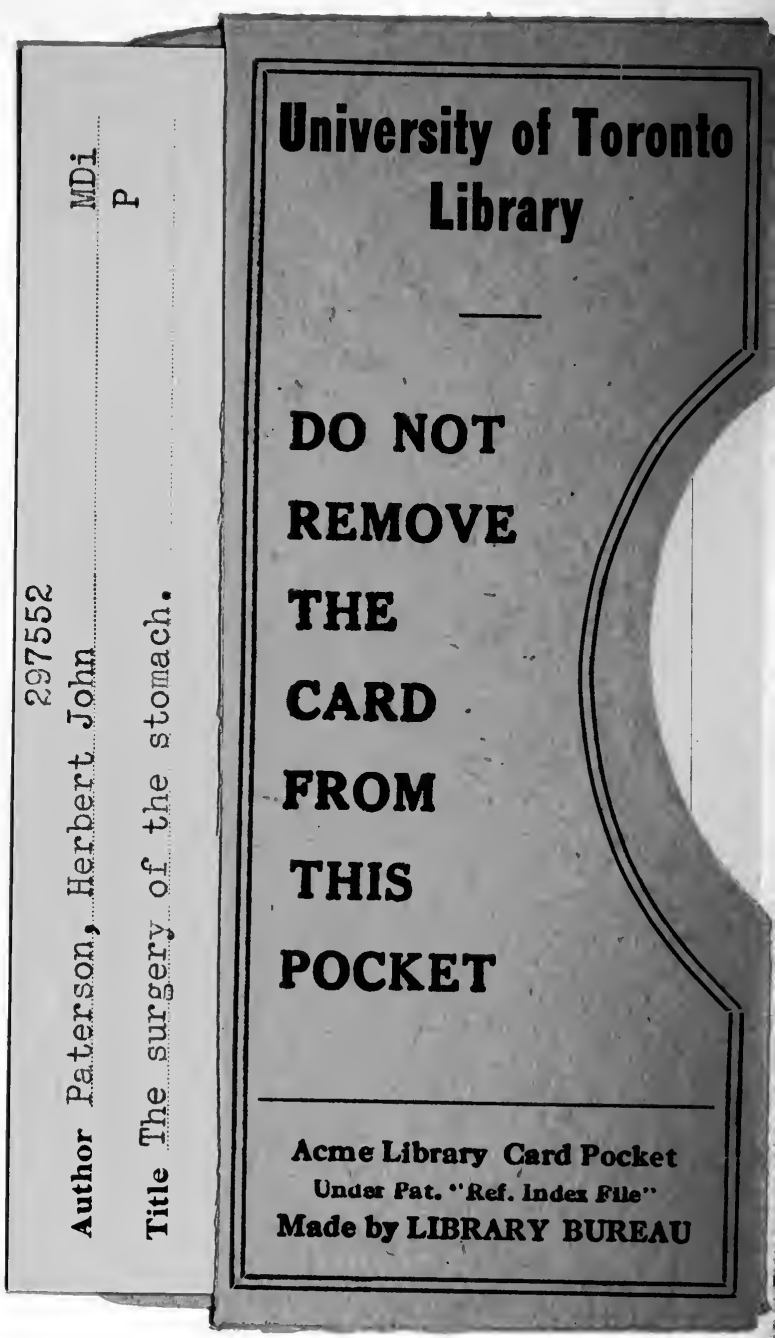


Why.

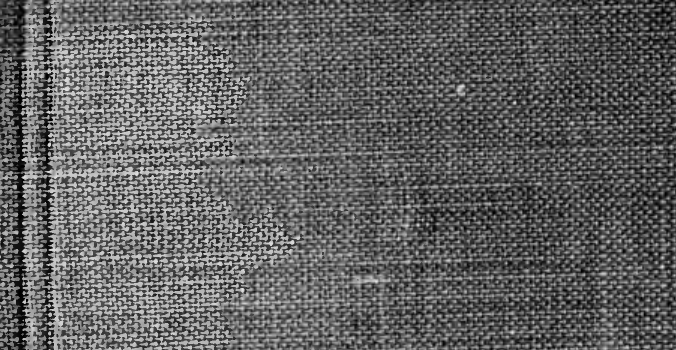

